\title{
Priority Based Resource Management Techniques for Distributed
}

\author{
Systems
}

By

\section{Ronnie Farrell, B.Eng. (EE)}

A thesis submitted to the Faculty of Graduate and Postdoctoral Affairs in partial

fulfillment of the requirement for the degree of

\section{Master of Applied Science in \\ Electrical and Computer Engineering}

Ottawa-Carleton Institute for Electrical and Computer Engineering

Department of Systems and Computer Engineering

Faculty of Engineering

Carleton University

Ottawa, Ontario, Canada

May 2013

C Copyright 2013, Ronnie Farrell 
Library and Archives

Canada

Published Heritage

Branch

395 Wellington Street

Ottawa ON K1A ON4

Canada
Bibliothèque et

Archives Canada

Direction du

Patrimoine de l'édition

395 , rue Wellington

Ottawa ON K1A ON4

Canada
Your file Votre référence

ISBN: 978-0-494-94670-1

Our file Notre référence

ISBN: 978-0-494-94670-1
NOTICE:

The author has granted a nonexclusive license allowing Library and Archives Canada to reproduce, publish, archive, preserve, conserve, communicate to the public by telecommunication or on the Internet, loan, distrbute and sell theses worldwide, for commercial or noncommercial purposes, in microform, paper, electronic and/or any other formats.

The author retains copyright ownership and moral rights in this thesis. Neither the thesis nor substantial extracts from it may be printed or otherwise reproduced without the author's permission.
AVIS:

L'auteur a accordé une licence non exclusive permettant à la Bibliothèque et Archives Canada de reproduire, publier, archiver, sauvegarder, conserver, transmettre au public par télécommunication ou par l'Internet, prêter, distribuer et vendre des thèses partout dans le monde, à des fins commerciales ou autres, sur support microforme, papier, électronique et/ou autres formats.

L'auteur conserve la propriété du droit d'auteur et des droits moraux qui protege cette thèse. $\mathrm{Ni}$ la thèse ni des extraits substantiels de celle-ci ne doivent être imprimés ou autrement reproduits sans son autorisation.
In compliance with the Canadian Privacy Act some supporting forms may have been removed from this thesis.

While these forms may be included in the document page count, their removal does not represent any loss of content from the thesis.
Conformément à la loi canadienne sur la protection de la vie privée, quelques formulaires secondaires ont été enlevés de cette thèse.

Bien que ces formulaires aient inclus dans la pagination, il n'y aura aucun contenu manquant. 


\section{Abstract}

This thesis focuses on priority based resource management. Matchmaking, involving the allocation of requests to resources is a key problem in the context of resource management on distributed systems including grids and clouds. To provide quality of service (QoS) guarantees to different clients, it is crucial to support Advance Reservation (AR) requests. Most existing works do not associate priorities with ARs. Sometimes these dynamic systems also require emergency response that demand immediate action. This thesis focuses on matchmaking strategies for handling AR requests with multiple priorities, as well as Emergency requests with the purpose of achieving high system performance. Accommodating a high priority AR request can lead to the abortion of low priority AR requests, whereas the arrival of an Emergency request may require the preemption of an executing request. This research includes the investigation of four abortion and four pre-emption policies. A performance analysis based on simulations is conducted. A comprehensive analysis of the results leading to key insights into system behaviour and performance is presented. 


\section{Acknowledgements}

Firstly, I am grateful to Cistel Technology Ottawa, and the Ontario Centres of Excellence that provided financial support for this research. I would especially like to give thanks and gratitude to my supervisor Professor Shikharesh Majumdar for his guidance, continuous support, and encouragement throughout this thesis. I sincerely appreciate his guidance and patience during every step of this thesis.

Next, I would like to thank Orlando for helping me get started with the GridSim toolkit.

Finally, I would like to give thanks to my family and friends for their support and encouragement. 


\section{Table of Contents}

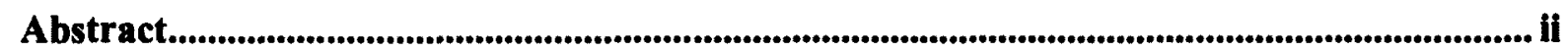

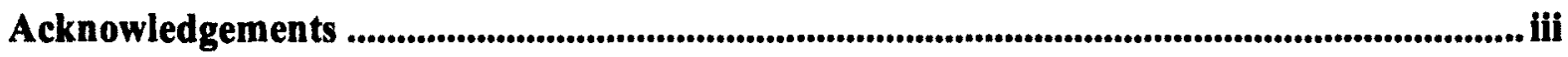

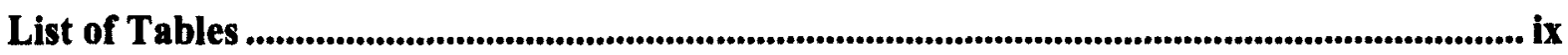

List of Figures.................................................................................................................................................. $x$

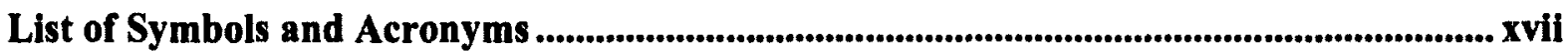

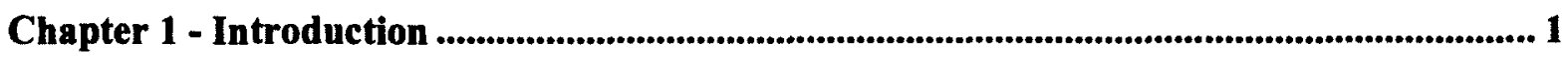

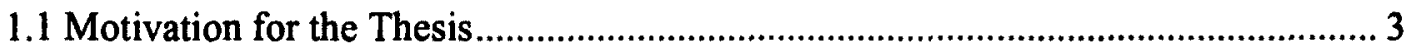

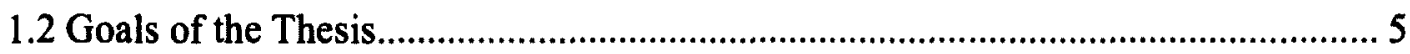

1.3 Contributions of the Thesis ....................................................................... 6

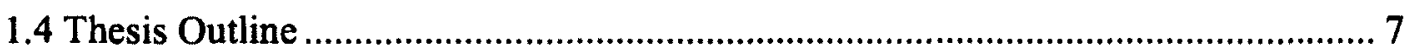

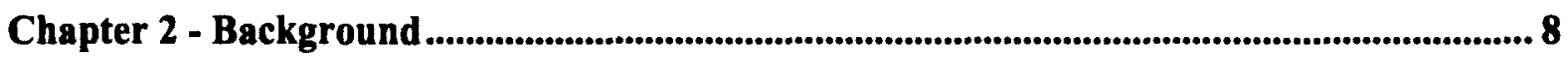

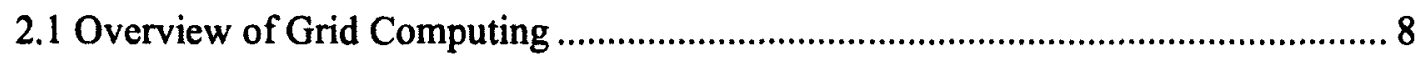

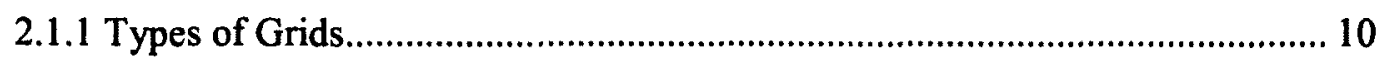

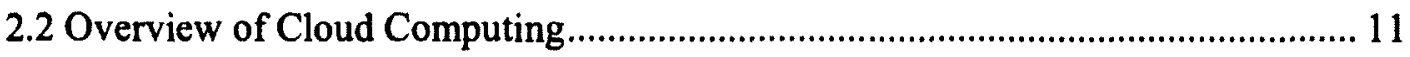

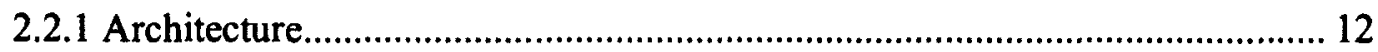

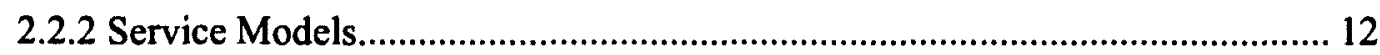

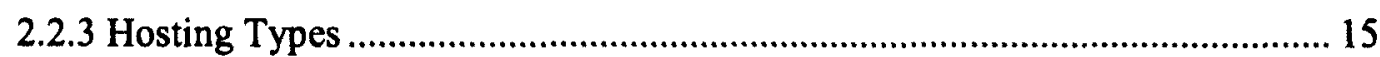

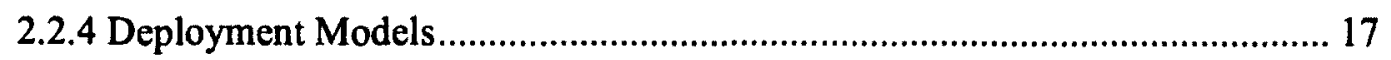

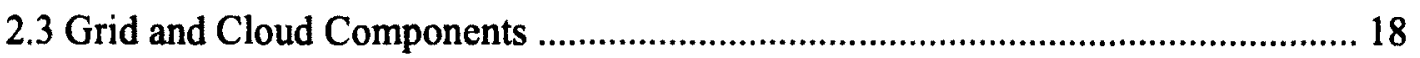

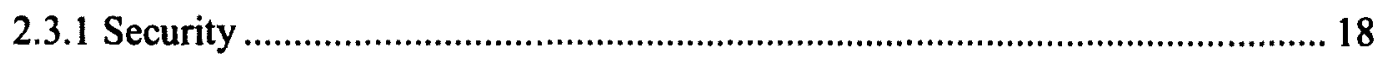


2.3.3 Scheduling 19

2.3.4 Resource Management. 20

2.3.5 Data Virtualization and Data Management. 20

2.3.6 Charge-back 20

2.4 Grid and Cloud Workloads 21

2.4.1 Request Types 21

2.4.2 Workload Parameters. 22

2.5 Related Work on Resource Management 23

2.5.1 Matchmaking and Scheduling in Grids and Clouds 23

2.5.2 Advanced Reservations. 27

2.5.3 Advance Reservations with Priorities. 32

Chapter 3 - Resource Management Techniques ............................................................... 35

3.1 Matchmaking Based on Knowledge of Scheduling Policies 35

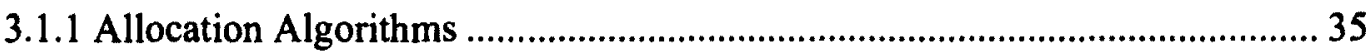

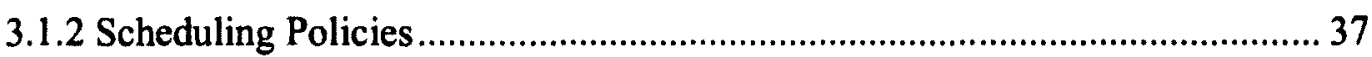

3.2 Handling Advanced Reservation Requests with Priorities ............................... 37

3.2.1 Advance Reservation Priority Level ....................................................... 38

3.2.2 Matchmaking Based on AR Request Priority Level................................... 39

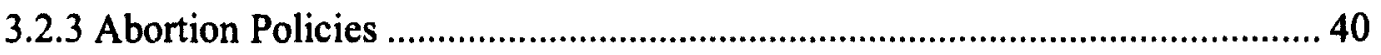


3.3 Matchmaking Strategy for Handling Emergency Requests.................................... 46

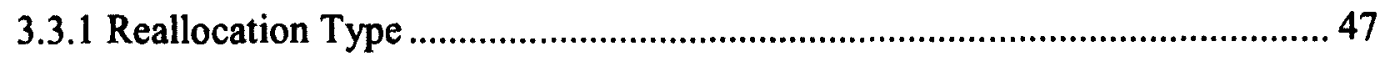

3.3.2 Known / Unknown Emergency Service Time .................................................. 47

3.3.3 Pre-emption Policies .................................................................................. 48

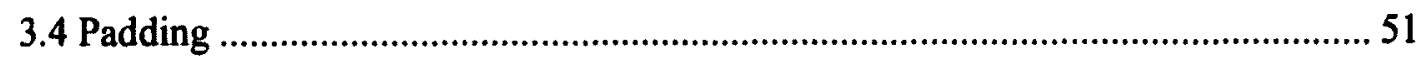

3.4.1 Next Request Start Time ........................................................................... 52

Chapter 4 - Simulation Model ........................................................................................................................ 53

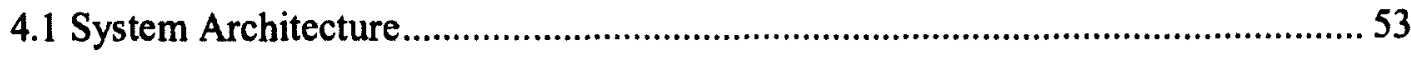

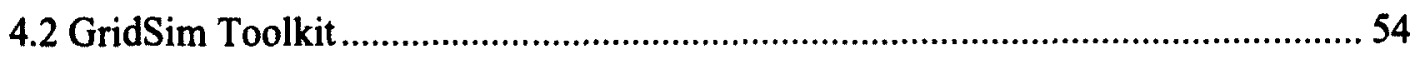

4.3 Simulation Entities........................................................................................... 55

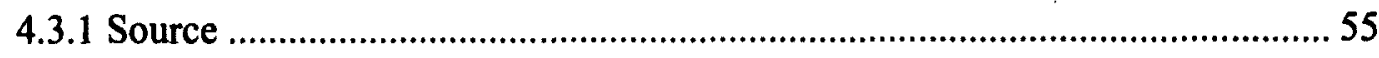

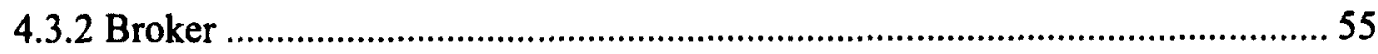

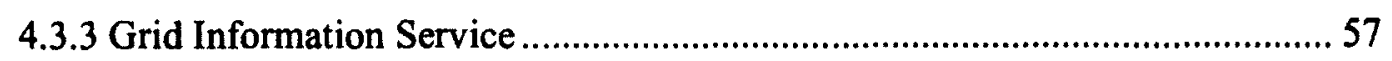

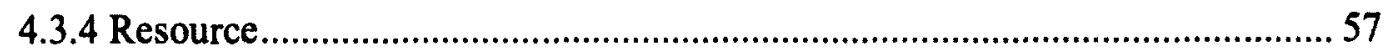

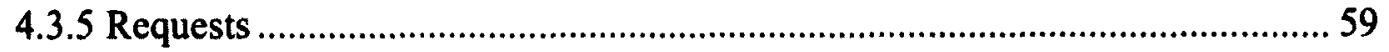

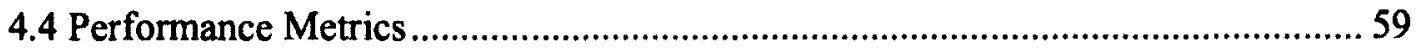

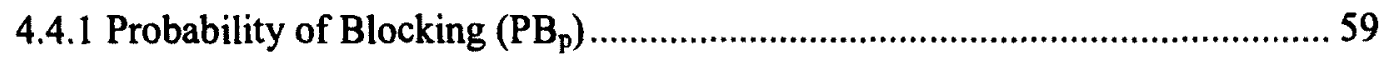

4.4.2 Probability of Aborting (PA) ……............................................................. 60

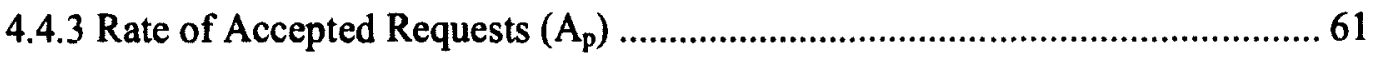

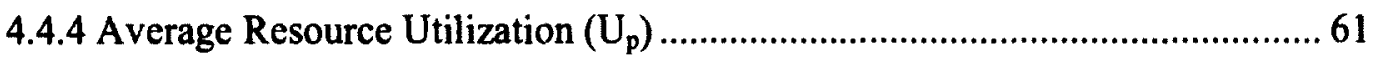


4.5 Workload and System Parameters 62

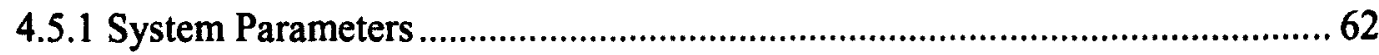

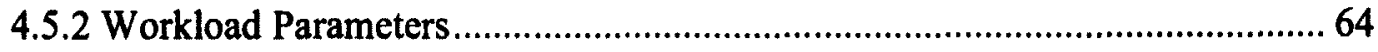

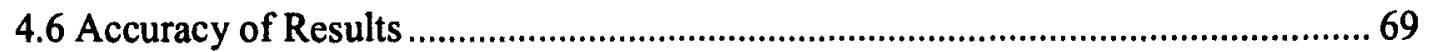

Chapter 5 - Performance Evaluation of the Resource Management Techniques.................. 70

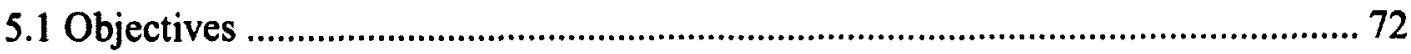

5.2 Performance of a System Containing ARs with Multiple Priority Levels.............. 74

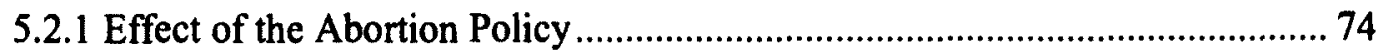

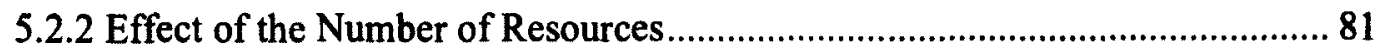

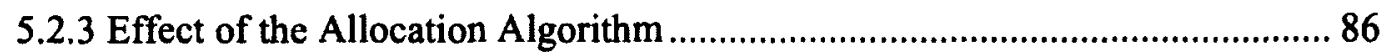

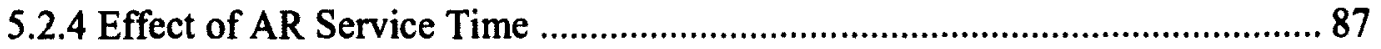

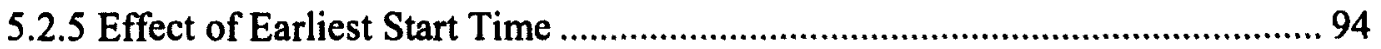

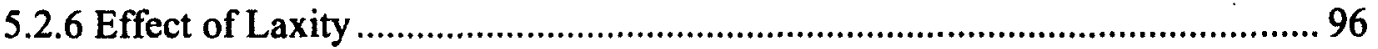

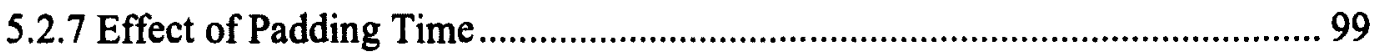

5.2.8 Effect of the Proportion of High Priority AR Requests ............................... 102

5.3 Performance of a System with Emergencies .................................................. 104

5.3.1 Effect of the Pre-emption Policy ................................................................ 104

5.3.2 Effect on the Number of Resources ................................................... 111

5.3.3 Effect of the Knowledge of the Emergency Service Time ......................... 115

5.3.4 Effect of Emergency Service Time ....................................................... 118 
5.3.6 Effect of Padding Time ............................................................................ 125

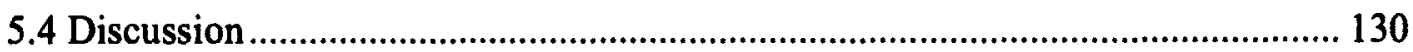

Chapter 6 - Conclusions ................................................................................................................................. 132

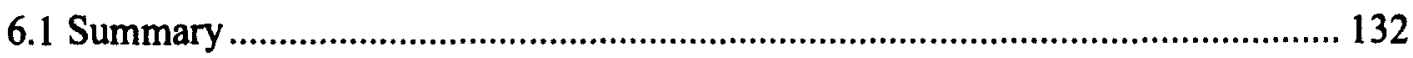

6.2 Insights into System Behaviour ....................................................................... 134

6.2.1 Impact of the Resource Management Techniques ......................................... 134

6.2.2 Impact of System and Workload Parameters on Performance ....................... 135

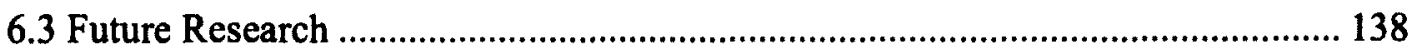

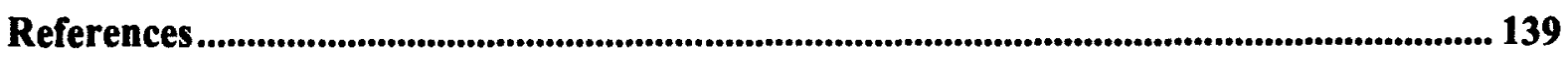

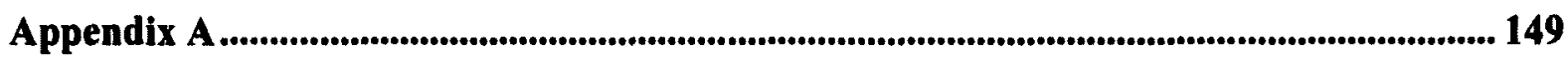

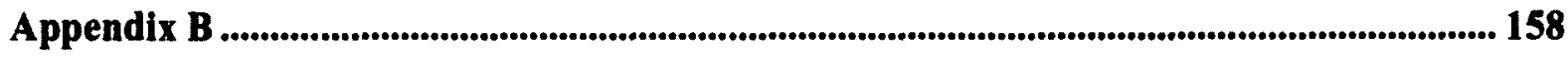




\section{List of Tables}

Table 4-1: Default Values for Key Resource Characteristics Provided by GridSim......... 58

Table 5-1: Default Values of System and Workload Parameters ........................................ 71

Table 5-2: List of Key Symbols.................................................................................... 73 


\section{List of Figures}

Figure 1-1: Structural View of a Grid/Cloud System ........................................................ 4

Figure 2-1: Cloud Architecture (based on [OXY-12]) …………………....................... 12

Figure 2-2: Infrastructure Hosting Types (based on [BAR-12]) ….................................. 16

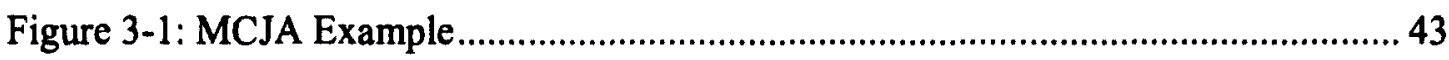

Figure 3-2: Model of AR Request with Padding ………............................................. 52

Figure 4-1: General System Architecture .................................................................... 54

Figure 4-2: GridSim Architecture (from [BUY-02]) ..................................................... 56

Figure 5-1: Impact of the Abortion Policies on the Probability of Blocking Low Priority

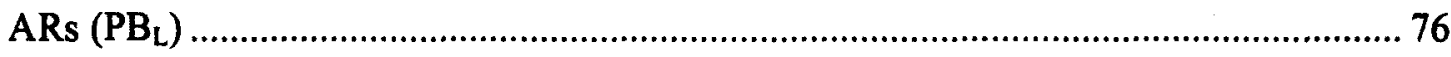

Figure 5-2: Impact of the Abortion Policies on the Probability of Blocking High Priority

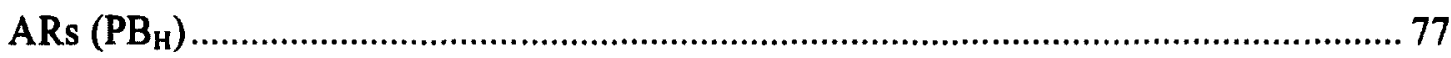

Figure 5-3: Impact of the Abortion Policies on the Probability of Aborting Low Priority

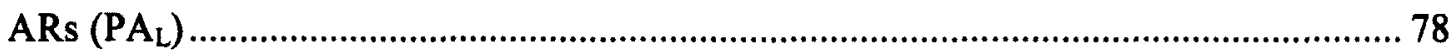

Figure 5-4: Impact of the Abortion Policies on the Average Resource Utilization due to

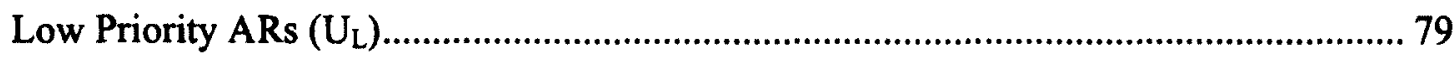

Figure 5-5: Impact of the Abortion Policies on the Average Resource Utilization due to

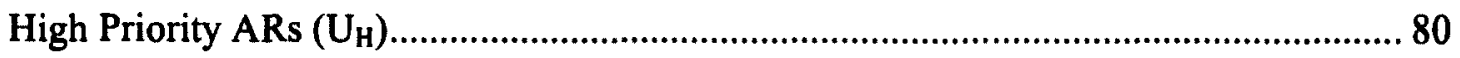

Figure 5-6: Impact of the Abortion Policies on the Overall Average Resource Utilization

$\left(\mathrm{U}_{\mathrm{T}}\right)$

Figure 5-7: Impact of the Number of Resources (R) on the Probability of Blocking Low

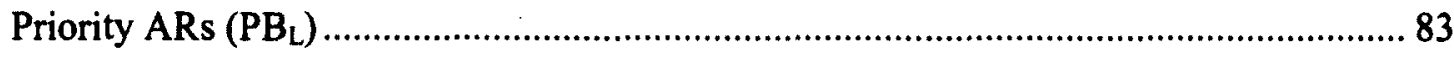


Figure 5-8: Impact of the Number of Resources (R) on the Probability of Blocking High Priority ARs $\left(\mathrm{PB}_{\mathrm{H}}\right)$ 83

Figure 5-9: Impact of the Number of Resources (R) on the Probability of Aborting Low

Priority ARs $\left(\mathrm{PA}_{\mathrm{L}}\right)$ 84

Figure 5-10: Impact of the Number of Resources (R) on the Average Resource Utilization due to Low Priority ARs $\left(\mathrm{U}_{\mathrm{L}}\right)$. 85

Figure 5-11: Impact of the Number of Resources ( $R$ ) on the Average Resource Utilization due to High Priority ARs $\left(\mathrm{U}_{\mathrm{H}}\right)$ 86

Figure 5-12: Impact of the Allocation Algorithm on the Probability of Aborting Low

Priority ARs $\left(\mathrm{PA}_{\mathrm{L}}\right)$

Figure 5-13: Impact of the Variability Factor for ARs $\left(V_{f, A R}\right)$ on the Probability of Blocking High Priority $\mathrm{ARs}\left(\mathrm{PB}_{\mathrm{H}}\right)$ 89

Figure 5-14: Impact of the Variability Factor for ARs $\left(\mathrm{V}_{\mathrm{f}, \mathrm{AR}}\right)$ on the Probability of Aborting Low Priority ARs $\left(\mathrm{PA}_{\mathrm{L}}\right)$

Figure 5-15: Impact of the Variability Factor for ARs $\left(V_{f, A R}\right)$ on the Average Resource Utilization due to High Priority ARs $\left(\mathrm{U}_{\mathrm{H}}\right)$ 91

Figure 5-16: Impact of the Mean AR Service Time on the Probability of Blocking High Priority ARs $\left(\mathrm{PB}_{\mathrm{H}}\right)$ 92

Figure 5-17: Impact of the Mean AR Service Time on the Probability of Aborting Low Priority ARs $\left(\mathrm{PA}_{\mathrm{L}}\right)$

Figure 5-18: Impact of the Mean AR Service Time on the Average Resource Utilization due to High Priority ARs $\left(\mathrm{U}_{\mathrm{H}}\right)$ 93

Figure 5-19: Impact of $\mathrm{T}_{\mathrm{s}}$ on the Probability of Blocking High Priority ARs $\left(\mathrm{PB}_{\mathrm{H}}\right) \ldots \ldots . .95$ 
Figure 5-20: Impact of $T_{s}$ on the Probability of Aborting Low Priority ARs (PA $)$....... 96 Figure 5-21: Impact of $T_{\mathrm{s}}$ on the Average Resource Utilization due to High Priority ARs $\left(\mathrm{U}_{\mathrm{H}}\right)$ 96

Figure 5-22: Impact of Laxity (L) on the Probability of Blocking High Priority ARs $\left(\mathrm{PB}_{\mathrm{H}}\right)$. 97

Figure 5-23: Impact of Laxity (L) on the Probability of Aborting Low Priority ARs (PA $)$

Figure 5-24: Impact of Laxity (L) on the Average Resource Utilization due to High

Priority ARs $\left(\mathrm{U}_{\mathrm{H}}\right)$ .99

Figure 5-25: Impact of Padding on the Probability of Blocking High Priority ARs $\left(\mathrm{PB}_{\mathrm{H}}\right)$ 100

Figure 5-26: Impact of Padding on the Probability of Aborting Low Priority ARs (PA $)$

Figure 5-27: Impact of Padding on the Overall Average Resource Utilization $\left(U_{T}\right) \ldots . .102$ Figure 5-28: Impact of the Proportion of High Priority ARs $\left(\mathrm{PAR}_{H}\right)$ on the Probability of

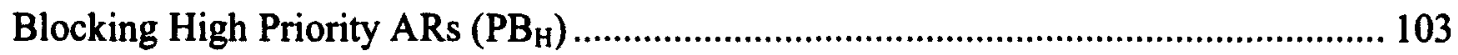
Figure 5-29: Impact of the Proportion of High Priority ARs $\left(\mathrm{PAR}_{H}\right)$ on the Probability of Aborting Low Priority ARs $\left(\mathrm{PA}_{\mathrm{L}}\right)$ 104

Figure 5-30: Impact of the Proportion of High Priority ARs $\left(\mathrm{PAR}_{H}\right)$ on the Average Resource Utilization due to High Priority ARs $\left(\mathrm{U}_{\mathrm{H}}\right)$. 105 Figure 5-31: Impact of the Pre-emption Policies on the Average Resource Utilization due to Emergencies $\left(\mathrm{U}_{\mathrm{E}}\right)$ 107 
Figure 5-32: Impact of the Pre-emption Policies on the Average Resource Utilization due to High Priority ARs $\left(\mathrm{U}_{\mathrm{H}}\right)$ 108

Figure 5-33: Impact of the Pre-emption Policies on the Average Resource Utilization due to Low Priority ARs $\left(\mathrm{U}_{\mathrm{L}}\right)$. 109

Figure 5-34: Impact of the Pre-emption Policies on the Probability of Aborting ARs because of Emergencies (PA-E) 110

Figure 5-35: Impact of the Number of Resources (R) on the Probability of Aborting Low Priority ARs because of Emergencies (PA-E) 112 Figure 5-36: Impact of the Number of Resources (R) on the Average Resource Utilization due to Low Priority ARs $\left(\mathrm{U}_{\mathrm{L}}\right)$.

Figure 5-37: Impact of the Number of Resources (R) on the Average Resource Utilization due to High Priority ARs $\left(\mathrm{U}_{\mathrm{H}}\right)$. 114 Figure 5-38: Impact of the Number of Resources $(R)$ on the Average Resource Utilization due to Emergencies $\left(\mathrm{U}_{\mathrm{E}}\right)$

Figure 5-39: Impact of Knowing the Emergency Service Time on the Probability of Aborting ARs becuase of Emergencies (PA-E) 116 Figure 5-40: Impact of the Reallocation Type on the Probability of Aborting ARs because of Emergencies (PA-E).

Figure 5-41: Impact of the Variability Factor for Emergencies $\left(\mathrm{V}_{\mathrm{f}, \mathrm{E}}\right)$ on the Probability of Aborting ARs because of Emergencies (PA-E).

Figure 5-42: Impact of the Mean Emergency Service Time on the Average Resource Utilization due to Emergencies $\left(\mathrm{U}_{\mathrm{E}}\right)$ 
Figure 5-43: Impact of the Mean Emergency Service Time on the Average Resource Utilization due to High Priority ARs $\left(\mathrm{U}_{\mathrm{H}}\right)$

Figure 5-44: Impact of the Mean Emergency Service Time on the Overall Rate of Accepted Requests $\left(\mathrm{A}_{\mathrm{T}}\right)$

Figure 5-45: Impact of the Proportion of Emergencies (PE) on the Probability of Aborting ARs because of Emergencies (PA-E)

Figure 5-46: Impact of the Proportion of Emergencies (PE) on the Average Resource Utilization due to Emergencies $\left(\mathrm{U}_{\mathrm{E}}\right)$.

Figure 5-47: Impact of the Proportion of Emergencies (PE) on the Average Resource Utilization due to High Priority ARs $\left(\mathrm{U}_{\mathrm{H}}\right)$

Figure 5-48: Impact of Padding on the Probability of Aborting ARs because of

Emergencies (PA-E) 126

Figure 5-49: Impact of Padding on the Overall Average Resource Utilization $\left(\mathrm{U}_{\mathrm{T}}\right)$..... 127

Figure 5-50: Impact of Next Request's Start Time on the Probability of Aborting ARs because of Emergencies (PA-E) 128

Figure 5-51: Impact of Next Request's Start Time on the Overall Average Resource Utilization $\left(\mathrm{U}_{\mathrm{T}}\right)$ 129

Figure A-1: Impact of the Abortion Policies on the Overall Probability of Blocking $\left(\mathrm{PB}_{\mathrm{T}}\right)$

Figure A-2: Impact of Variability Factor for ARs $\left(\mathrm{V}_{\mathrm{f}, \mathrm{AR}}\right)$ on the Probability of Blocking Low Priority $\mathrm{ARs}\left(\mathrm{PB}_{\mathrm{L}}\right)$. 149

Figure A-3: Impact of Variability Factor for $A R s\left(V_{f, A R}\right)$ on the Overall Rate of Accepted Requests $\left(\mathrm{A}_{\mathrm{T}}\right)$ 150 
Figure A-4: Impact of Variability Factor for ARs $\left(V_{f, A R}\right)$ on the Average Resource Utilization due to Low Priority ARs $\left(\mathrm{U}_{\mathrm{L}}\right)$ 150

Figure A-5: Impact of the Mean AR Service Time on the Probability of Blocking Low

Priority ARs $\left(\mathrm{PB}_{\mathrm{L}}\right)$

Figure A-6: Impact of the Mean AR Service Time on the Overall Rate of Accepted

Requests $\left(\mathrm{A}_{\mathrm{T}}\right)$

Figure A-7: Impact of the Mean AR Service Time on the Average Resource Utilization

due to Low Priority ARs $\left(\mathrm{U}_{\mathrm{L}}\right)$. 152

Figure A-8: Impact of $T_{s}$ on the Probability of Blocking Low Priority ARs ( $\left.\mathrm{PB}_{\mathrm{L}}\right) \ldots \ldots .152$

Figure A-9: Impact of $T_{s}$ on the Overall Rate of Accepted Requests $\left(A_{T}\right)$.

Figure A-10: Impact of $T_{s}$ on the Average Resource Utilization due to Low Priority ARs

$\left(\mathrm{U}_{\mathrm{L}}\right)$

Figure A-11: Impact of Laxity (L) on the Probability of Blocking Low Priority ARs

Figure A-12: Impact of Laxity (L) on the Average Resource Utilization due to Low

Priority ARs $\left(U_{L}\right)$

Figure A-13: Impact of Padding on the Probability of Blocking Low Priority ARs (PB

Figure A-14: Impact of Padding on the Overall Rate of Accepted Requests $\left(A_{T}\right)$ 155

Figure A-15: Impact of Padding on the Average Resource Utilization of High Priority

ARs $\left(\mathrm{U}_{\mathrm{H}}\right)$

Figure A-16: Impact of the Proportion of High Priority ARs $\left(\mathrm{PAR}_{H}\right)$ on the Probability of Blocking Low Priority ARs $\left(\mathrm{PB}_{\mathrm{L}}\right)$ 156 
Figure A-17: Impact of the Proportion of High Priority ARs $\left(\mathrm{PAR}_{\mathbf{H}}\right)$ on the Average

Resource Utilization due to Low Priority ARs $\left(\mathrm{U}_{\mathrm{L}}\right)$

Figure B-1: Impact of the Pre-emption Policies on the Probability of Blocking Low

Priority ARs $\left(\mathrm{PB}_{\mathrm{L}}\right)$.

Figure B-2: Impact of the Pre-emption Policies on the Probability of Blocking High

Priority ARs $\left(\mathrm{PB}_{\mathrm{H}}\right)$

Figure B-3: Impact of the Pre-emption Policies on the Overall Average Resource

Utilization $\left(\mathrm{U}_{\mathrm{T}}\right)$

Figure B-4: Impact of the Number of Resources (R) on the Overall Rate of Accepted

Requests $\left(\mathrm{A}_{T}\right)$

Figure B-5: Impact of the Mean Emergency Service Time on the Average Resource

Utilization due to Low Priority ARs $\left(\mathrm{U}_{\mathrm{L}}\right)$

Figure B-6: Impact of the Proportion of Emergencies (PE) on the Average Resource

Utilization due to Low Priority ARs $\left(\mathrm{U}_{\mathrm{L}}\right)$

Figure B-7: Impact of Padding on the Average Resource Utilization due to Emergencies

$\left(\mathrm{U}_{\mathrm{E}}\right)$ 


\section{List of Symbols and Acronyms}

Note: Symbols related to the simulation results are presented in Table 5-2.

$\lambda$

ACO

AFCFS

AppLeS

AR

$\mathrm{AR}_{\mathrm{L}}$

$\mathrm{AR}_{\mathrm{H}}$

ARM

ARWP

AS

CPU

$\mathrm{CW}$

D

DPR

DPSAR

DVM

$\mathrm{E}$

EC2

EDF

EFT

EW

FCFS
Arrival Rate

Ant Colony Optimization

Adaptive-First-Come-First-Served

Application Level Scheduling

Advanced Reservation

Low Priority Advance Reservation Request (Priority Level 0)

High Priority Advance Reservation Request (Priority Level 1)

Advance Reservation Module

Advance Reservation Without Priority Algorithm

Any Schedulability

Central Processing Unit

Conflict Window

Deadline

Dynamic Priority Resolution

Dynamic Priority Scheduler for Advance Reservation

Dispatcher Virtual Machine

Emergency Request (Priority Level 2)

Elastic Compute Cloud

Earliest Deadline First

Expected Finish Time

Execution Window

First-Come-First-Served 
FFAA

GAE

GARA

GASS

GIS

GRAM

GridARS

GS-A

GS-P

GSI

GSP

laaS

IT

JVM

LBACO

LJFS

LS-A

LS-P

MCJA

N

NA-A

NFAA

NR-A

NR-P

OD
First Fit Allocation Algorithm

Google App Engine

Globus Architecture for Reservation and Allocation

Grid Access to Secondary Storage

Grid Information Service

Grid Resource Allocation Manager

Grid Advance Reservation-based System

Greatest Sum of Service Times Abortion Policy

Greatest Service Time Pre-emption Policy

Grid Security Infrastructure

Gridlet Sorting Policy

Infrastructure as a Service

Information Technology

Java Virtual Machine

Load Balancing Ant Colony Optimization

Largest-Job-First-Served

Largest Sum of Slack Times Abortion Policy

Largest Slack Pre-emption Policy

Minimum Conflicting Jobs Algorithm

Number of Requests

Next Abortable Resource Abortion Policy

Next Fit Allocation Algorithm

Next Resource Abortion Policy

Next Resource Pre-emption Policy

On Demand 


$\begin{array}{ll}\text { PaaS } & \text { Platform as a Service } \\ \text { PoS } & \text { Probability of Success } \\ \text { RARP } & \text { Relaxed Advance Reservation Policy } \\ \text { RB } & \text { Resource Broker Algorithm } \\ \text { RM } & \text { Resource Manager } \\ \text { RMS } & \text { Resource Management System } \\ \text { RSPB } & \text { Reservation Scheduler with Priorities and Benefit Functions } \\ \text { SP } & \text { Resource Scheduling Policy } \\ \text { SaaS } & \text { Software as a Service } \\ \text { SLA } & \text { Service Level Agreement } \\ \text { SQF } & \text { Shortest Queue First } \\ \text { ST-P } & \text { Shortest Time Pre-emption Policy } \\ \text { QoS } & \text { Quality of Service } \\ \text { T } & \text { Start Time of an AR Request } \\ \text { VM } & \text { Virtual Machine } \\ \text { VO } & \text { Virtual Organization } \\ \text { WNS } & \text { Weather Network Service } \\ \text { XSEDE } & \end{array}$




\section{Chapter 1 - Introduction}

Distributed systems such as grids, and more recently clouds are being used for enterprise and scientific computing. As applications are becoming more sophisticated with the advances in technology, their size and complexity increase along with the amount of computational hardware required to run it. Often the requirements of these applications surpass the resource's capacity existing within a single site. It is extremely expensive for a sole organization to maintain dedicated resource capabilities that can handle the increase of the demands that these applications require. Organizations have steered towards a distributed computing architecture and away from a centralized computing architecture to accommodate these challenges and the evolving demands. Distributed computing gives rise to a more scalable solution to the issues faced from applications that are resource intensive. As the progress of development in Internet technologies improves resources distributed geographically become more accessible with nominal cost for distributed computing.

The Grid evolved as a result of distributed computing. The Grid emerged from the demands to share data, software and a variety of other types of resources that were required by scientific institutions for advance science and engineering [FOS-01]. A virtual organization (VO) is responsible for controlling the sharing of resources. The VO is characterized as a set of resources and/or institutions bound by the sharing rules. These rules define what can be shared, who is permitted to share, and the conditions under which sharing is allowed [FOS-01]. Ian Foster, one of the pioneers of Grid computing, defined the Grid as a system that "coordinates resources that are not subject to centralized 
control, uses standard, open, general-purpose protocols and interfaces, and delivers nontrivial qualities of service" [FOS-02].

Initially the grid was mainly used by institutions interested in carrying out research on large amounts of data. Over the past few years a more business approach to grid computing has given rise to what is now known as cloud computing [BUY-09]. Cloud and grid computing share a similar vision including the reduction of computing costs, increased reliability, and increased flexibility by revolutionizing computers from something that is purchased and operated by oneself to something that is operated by a third party, but there are some distinctions that make the cloud an extension of the grid [FOS-08]. There are several definitions of what cloud computing really encompasses, but most tend to focus on specific aspects of the technology. In [VAQ-08] the authors studied more than 20 definitions to determine a consensus definition containing the essential characteristics, defining a cloud as "a large pool of easily usable and accessible virtualized resources (such as hardware, development platforms and/or services). These resources can be dynamically configured to adjust variable load (scale), allowing also for optimum resource utilization. This pool of resources is typically exploited by a pay-peruse model in which guarantees are offered by the Infrastructure Provider by means of customized Service Level Agreements (SLAs)" [VAQ-08]. The cloud is a concept that is still changing but the set of features that closely resemble the lowest common denominator of several definitions include scalability, pay-per-use utility model, and virtualization [VAQ-08]. 


\subsection{Motivation for the Thesis}

Grid and cloud systems involve the access to multiple distributed resources. In such an environment, resources may be added or removed dynamically. To ensure user satisfaction, it is necessary to consider the quality of service (QoS) requirements of different users. As a result of the uncertainties of resource availabilities in these dynamic systems, it is imperative to support Advance Reservation (AR) requests submitted by the users. This thesis focuses on satisfying the deadlines associated with ARs for different priority levels.

Along with Advance Reservations, matchmaking and scheduling are also important in attaining QoS on such systems. Various schedulers used for assigning requests to available resources have been proposed in the literature. Traditional schedulers such as first-come-first-served, round robin, shortest job first, etc., do not guarantee resource allocation for a specified time in the future. To solve this problem, a scheduling system for ARs has been proposed [FOS-01, SUL-04]. This scheduling system for ARs [SUL04] does not handle jobs belonging to various users that have different priorities, thus requiring different treatment. Along with [SUL-04], most existing works in the literature do not associate priorities with ARs. These priorities need to be resolved in a dynamic manner, making the resource management system more complex. Consequently, there is a demand for resource management techniques that are capable of handling the priorities of incoming $\mathrm{AR}$ requests.

Real world systems that are dynamic in nature, such as assistive healthcare [HOA-10] and bridge infrastructure management [MAJ-12], sometimes require emergency response. In these rare events, in order to handle the emergency which requires immediate 
attention, the matchmaker that is responsible for allocating requests needs to select a resource to execute the emergency on so that a high QoS is achieved. To the best of our knowledge, the literature does not address the effects that emergencies cause on a system involving ARs.

An example of such a system is shown in Figure 1-1. The figure includes the following components: Advance Reservation and Emergency requests which are submitted to the broker as a stream of requests from multiple users, a broker that performs matchmaking and matches a request to a resource, and a pool of resources that are capable of executing the request. In this type of system it is the broker's responsibility to perform matchmaking so that the required level of QoS is met. For systems containing ARs, the QoS requirement is typically found in the form of a deadline.

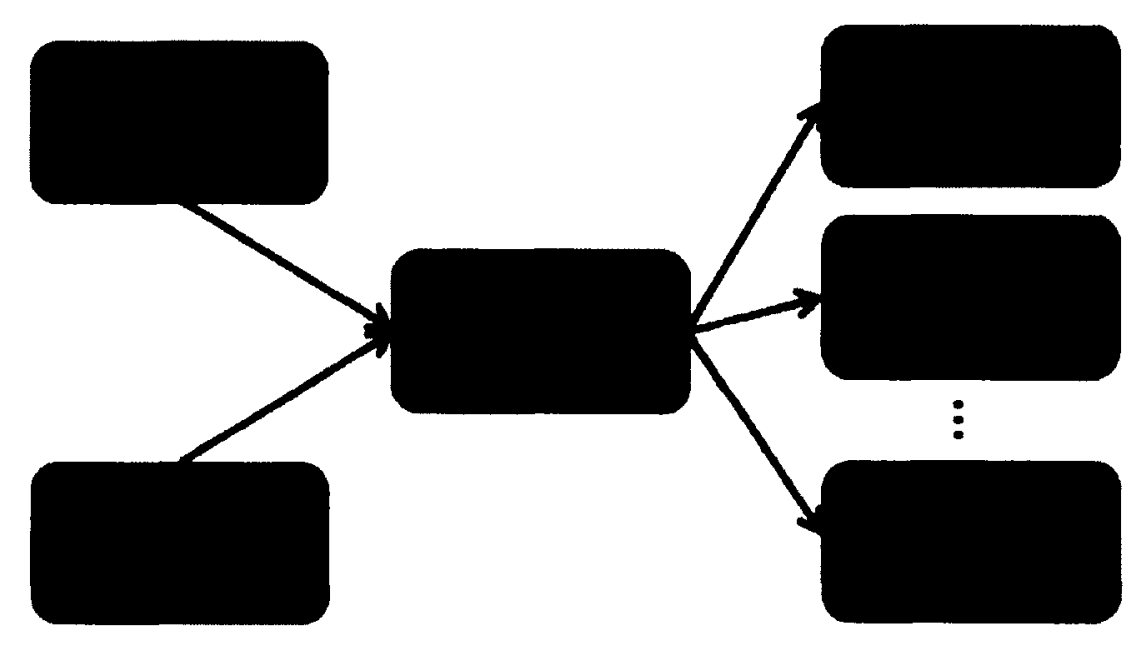

Figure 1-1: Structural View of a Grid/Cloud System

This thesis concerns matchmaking strategies that can be deployed at the broker for handling $\mathrm{AR}$ requests with various priorities and Emergency requests. It also involves a pre-emptive scheduler that is capable of accommodating higher priority requests where 
these requests would otherwise be rejected. Both abortion and pre-emption are used in the matchmaking strategies. Abortion leads to the removal of an already accepted AR request from the resource, whereas pre-emption refers to the interruption of an executing request. Depending on the resource availability, a pre-empted request can either be resumed or aborted. A further discussion of abortion and pre-emption are presented in Chapter 3.

\subsection{Goals of the Thesis}

The goal of this thesis is to devise a way of handing Emergency and Advance Reservation requests with priorities in a grid or cloud while attaining the highest level of QoS possible. To make this manageable, this overall goal is divided into sub-goals.

One sub-goal is devising a matchmaking technique that is capable of determining the schedulability of an AR request with a given priority level and effectively accommodating that request by allocating it on an appropriate resource. Two levels of priorities are handled giving rise to low and high priority AR requests.

Accommodating an emergency may lead to the abortion of an existing AR request (discussed in detail in Chapter 3). As a result, another sub-goal for handling emergency requests is the devising of matchmaking techniques that are capable of scheduling Emergency requests while minimizing the number of ARs that are aborted.

These resource management techniques are used by the broker which has knowledge of the local scheduling policy that was devised with the capabilities of aborting lower priority requests, as well as pre-empting requests to accommodate emergencies.

A simulation based investigation is conducted. The performance of the resource management techniques are evaluated using several performance metrics that are 
computed during the simulation runs. Various system and workload parameters are varied throughout the simulations. A significant goal of the thesis is to perform an in depth analysis on the performance of the resource management techniques, as well as determining the impact that the system and workload parameters have on the performance of the system.

\subsection{Contributions of the Thesis}

To the best of our knowledge, this is one of the first works on resource management for systems subjected to both AR requests with priorities and Emergency requests.

The main contributions of the thesis include:

- Devising of abortion policies which allow the broker to select an appropriate resource that is able to accommodate high priority AR requests that would otherwise be rejected. Insights on the performance of the proposed abortion policies (described in Section 3.2.3) are given.

- Devising of pre-emption policies which allow the broker to select a resource to execute an Emergency request that minimize the number of AR requests aborted. Insights on the performance of the pre-emption policies (described in Section 3.3.3) are presented.

- Devising of a priority scheduler that is capable of handling ARs with multiple priority levels.

- Insights into the impact of the various system and workload parameters on system behaviour and performance as captured through rigorous simulation experiments are presented. 


\subsection{Thesis Outline}

The remainder of this thesis is presented as follows. Chapter 2 provides an overview of grid and cloud computing, and presents related work on resource management. Chapter 3 describes the resource management techniques developed in this thesis for handling Emergency and Advanced Reservation requests with priorities. The simulation model implemented for performance analysis is explained in Chapter 4. Chapter 5 analyses the results acquired from the simulation experiments performed with the resource management techniques for various system and workload parameters on a system subjected to Emergency and Advance Reservation requests with priorities. Chapter 6 concludes the thesis and proposes directions for future research. 


\section{Chapter 2 - Background}

This chapter presents the concepts associated with Grid and Cloud computing. Based on existing literature, Section 2.1 and Section 2.2 provide an overview of grid and cloud computing respectively. The common components associated with these types of systems are described in Section 2.3. Section 2.4 discusses the various workloads of grids and clouds, and Section 2.5 presents a literature survey of the related work on resource management.

\subsection{Overview of Grid Computing}

The aim of the grid is to provide access to resources on demand in a similar way that one can connect an electronic device into a power outlet to obtain power. The term Grid was coined in the mid 1990's and was derived from the power grid system due to the infrastructure of the Grid being similar to that of the power grid [FOS-04]. The power is shared and distributed via inter-connected nodes. One of the pioneers of grid computing, Ian Foster, provided a three point checklist that has to be fulfilled for a system to constitute as a Grid. Pertaining to this checklist, a Grid is defined as a system that [FOS02]:

\section{Coordinates resources that are not subject to centralized control}

2. Uses standard, open, general-purpose protocols and interfaces

3. Delivers non-trivial qualities of service

The first point in the checklist indicates that a Grid integrates and coordinates resources belonging to different domains in order to manage the issues of security, policy, payment, and membership that occur in this type of setting [FOS-02]. A cluster management 
system such as Veridian's Portable Batch System [ALT-12] like a Grid bring resources together and are considered as a powerful Grid resource. However, it does not qualify as a Grid itself due to its centralized control of the resources it manages.

The second point emphasizes that a Grid, unlike an application-specific system, should have protocols and interfaces that handle the fundamental issues including authentication, authorization, resource discovery and resource access that are standardized and generalized so that they permit the sharing of resources from anyone interested [FOS-02]. Distributed computing systems such as Platform's Multi-Cluster [IBM-12] and Condor [SHA-07] like Grids do integrate distributed resources without centralized control, and provide appealing qualities of service. However, the protocols employed are too specialized and for the most part, not open or standard. Thus, these systems do not fully meet the criteria of the definition and can only be considered as a Grid in a limited way [FOS-02].

The last point dictates that a Grid permits its resources be employed in an coordinated fashion to provide various qualities of service (QoS). For example, the QoS may relate to response time, throughput, availability, security, and/or co-allocation of multiple types of resources to adhere to the demands of the user, so that the overall system performs significantly better than that of the sum of its parts [FOS-02]. Although the Web uses open, general-purpose protocols that support access to distributed resources, it is not considered a Grid because it does not provide for the coordinated use of resources to deliver non-trivial QoS. 


\subsubsection{Types of Grids}

Grids can be classified by the type of solutions they seek to provide. The three primary types of grids include computational grids, data grids, and service grids [KRA-02]. There are no set boundaries between these grid types and often grids may be a combination of two or more of these.

\subsubsection{Computational Grids}

Computational grids, the most commonly used grid type, focuses on allotting resources primarily for computational power in resource intensive applications. In these type of grids, the majority of machines are high performance servers. The resources are aggregated acting as a unified processing resource. Computational grids can be further subdivided into the categories of distributed supercomputing and high throughput based on how the resources are utilized [KRA-02]. The purpose of a distributed supercomputing grid is to decrease the completion time of a job by running the application in parallel on multiple machines. These applications aim at solving challenging problems such as weather modelling [KRA-02]. The intention of high throughput grids is to increase the completion rate of a stream of jobs. These are geared for parameter sweep type applications such as Monte Carlo experiments [KRA-02]. The Extreme Science and Engineering Discovery Environment (XSEDE) is an example of a computational grid which replaced and expands on the NSF TeraGrid project [EXT-12]. It reduces technological barriers to the access and use of computing resources.

\subsubsection{Data Grids}

Data grids are responsible for storing data and granting access to those authorized to share it. They supply an infrastructure that is distributed across several networks for 
creating new information from data repositories like data warehouses or digital libraries [KRA-02]. Unlike a computational grid, a data grid has a specialized infrastructure which is offered to applications for data access and storage management. The European DataGrid Project [DAT-12] is a practical application of a data grid. This is a project that is funded by the European Union aimed at building the next generation computing infrastructure that will facilitate the sharing of large-scale databases.

\subsubsection{Service Grids}

Service grids offer services provided by a collection of resources. They can be further broken down into collaborative, on-demand, and multimedia grids [KRA-02]. Collaborative grids enable real time interaction by connecting applications and users into collaborative workgroups through a virtual workspace. On demand grids dynamically aggregate various resources to offer new services. Multimedia grids supply the infrastructure for real-time multimedia applications.

\subsection{Overview of Cloud Computing}

Cloud computing is a relatively new paradigm which has gained enormous attention as it provides flexibility and scalability without investing upfront in the set up and operation of large scale computing infrastructures and data centres [BAB-11]. In [BUY-09] a cloud is defined as a type of distributed system comprised of a collection of virtualized and inter-connected computers that are dynamically provisioned and exposed as one or more unified computing resources based on service-level agreements (SLAs) agreed upon through negotiation between the consumer and service provider. The cloud is a concept that is still changing but the set of features that closely resemble the lowest common 
denominator of several definitions include scalability, pay-per-use utility model, and virtualization [VAQ-08].

\subsubsection{Architecture}

Cloud computing systems are divided into two parts known as the front end and the back end which are inter-connected via a network, typically the Internet as seen in Figure 2-1 [JAD-12]. The front end is what the client deals with and encompasses the client's computer and the application needed to access the cloud. The back end represents the cloud of the system and entails the cloud computing services such as various computers, servers, and data storage [JAD-12].

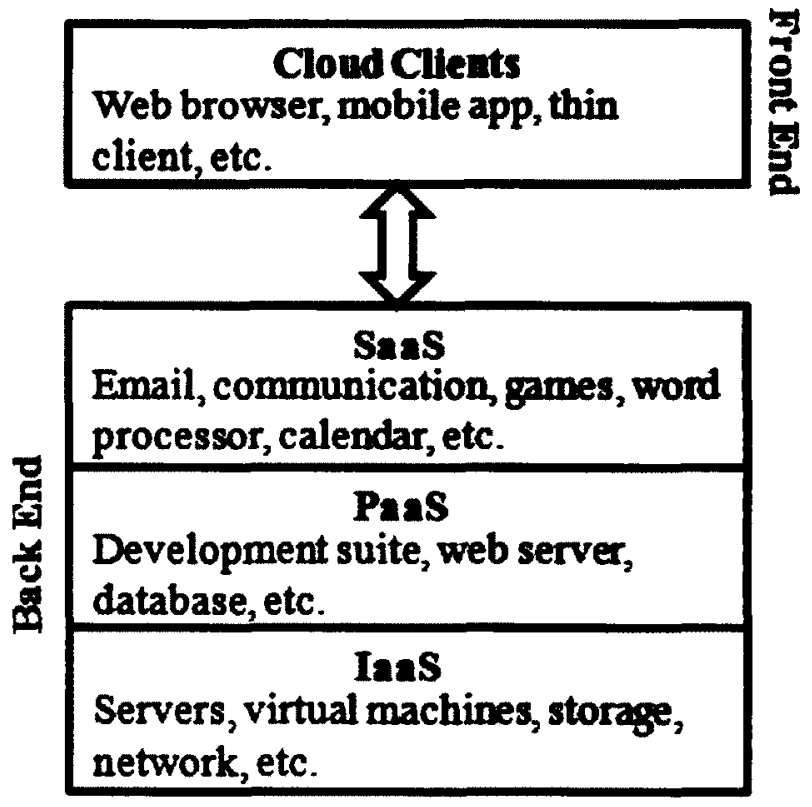

Figure 2-1: Cloud Architecture (based on [OXY-12])

\subsubsection{Service Models}

Cloud computing providers offer their services based on three fundamental models

known as Infrastructure as a Service (IaaS), Platform as a Service (PaaS), and Software 
as a Service (SaaS) [MAL-11]. The IaaS model is the most basic and all higher models abstract from the details of the lower models as observed in Figure 2-1. The cloud services are accessible as pay-as-you-go where the client only pays for the resources used for a specific time and the pricing for these cloud services typically vary according to QoS requirements [BUY-09]. All of these models allow users to run applications and store data online. However, each offers a different level of user flexibility and control.

\subsubsection{Infrastructure as a Service (IaaS)}

Infrastructure as a Service (IaaS) is the most basic model of cloud computing and offers the required physical infrastructure as a service. The client does not have to purchase any of the back end hardware such as servers, data centres, or network resources and only pays for the duration of time that the service is used [JAD-12]. This model is able to deploy and run any existing arbitrary software that the client wants on the service provider's computers. The software may be run on physical dedicated machines, or more frequently on virtual machines [MAL-11]. IaaS models commonly offer support for on demand scalability of computing and storage resources [BAB-11]. Amazon's Elastic Compute Cloud (EC2) [AMA-12], Rackspace Cloud [RAC-12], and Google Compute Engine [GOO-12] are examples of laaS. The Amazon EC2 provides resizable compute capacity in the cloud, and is designed to make web-scale computing easier for developers [AMA-12]. The Google Compute Engine offers the ability to launch virtual machines on-demand, manage network connectivity using a flexible networking solution, as well as accessing a variety of data storage alternatives from the virtual machines [GOO-12]. 


\subsubsection{Platform as a Service (PaaS)}

Platform as a Service (PaaS) offers a computing platform using the underlying cloud infrastructure. In PaaS, application developers have access to a development platform via its APIs which support certain programming languages [BAB-11]. It typically has all of the applications required by the user already deployed on it [JAD-12]. This provides users with the ability to run their created software on a cloud platform without the cost and hassle of purchasing and maintaining the hardware and software required for it. Google App Engine (GAE) [PRO-12] and Windows Azure [MIC-12] are examples of PaaS. GAE is a cloud computing platform supporting Python, Java, and Go for developing and hosting web applications in Google managed data centres. It offers automatic scaling to handle additional demand [PRO-12]. Windows Azure is a Microsoft cloud computing platform used to create and run applications of any language, tool, or framework through a global network of Microsoft data centres. It makes it possible for developers to integrate their public cloud applications with their existing IT environment [MIC-12].

\subsubsection{Software as a Service (SaaS)}

Within Software as a Service (Saas), service providers deploy and run off-the-shelf applications in the cloud and the users access them via the cloud clients [MAL-11]. This eliminates the need to install and operate applications on the users own computers making things easier for maintenance and support [JAD-12]. Thus, within this model users are not required to purchase the software or hardware, and only pay a rental fee giving them the benefits of the corresponding resources and maintenance services [WAN-10]. A drawback of this model is that the user does not have the ability to manage 
or control the infrastructure of the cloud [MAL-11]. Google Apps [WAN-10] is a wellknown example of SaaS. Google Apps provides various web applications with similar functionality as traditional office suites such as Gmail, Google Calendar, Talk, Docs, and Sites.

\subsubsection{Hosting Types}

The fundamental component of the cloud infrastructure is the server. A server can be either physical or virtual [BAR-12]. Physical servers are discrete, individual computers. In contrast, virtual server instances are software controlled slices of the physical servers shared amongst many users by a process called virtualization. There are four different ways in which the infrastructure can be hosted by the cloud service provider's data centre as shown in Figure 2-2. In each instance in the figure, the cloud data center is represented by a large rectangle consisting of both physical servers depicted by small rectangles and virtual server instances illustrated by circles. The physical servers or virtual server instances that are actually employed for a given hosting type are shaded in the figure. The rectangles and circles that are not filled in depict the other resources in the data centre that are not used for the given hosting type.

\subsubsection{Private Cloud Hosting}

As shown in Figure 2-2a, private cloud hosting is where the client rents a number of dedicated physical servers located in the same part of the data centre [BAR-12]. This allows the hardware to be kept separate from other users and is why private clouds are deemed to be the most secure. The downside to this type of hosting is that the block of servers cannot be dynamically scaled down as they are permanently dedicated to one client, and thus is the most expensive form of hosting [BAR-12]. 
a) Prtvate Clowd Hosthes

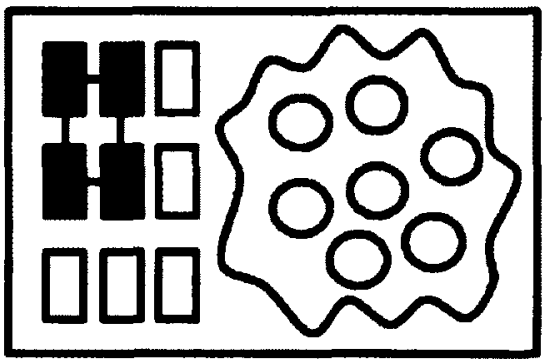

c) Hybrid Hostin:

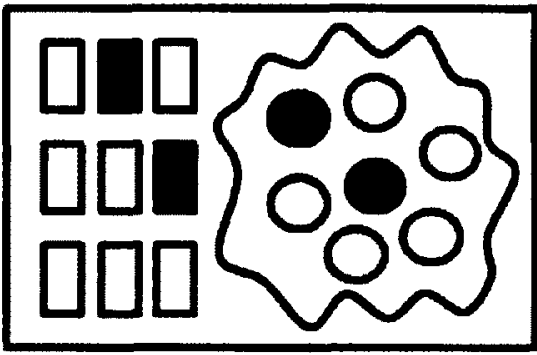

Physical Server (employed)

Physical Server (not in use) b) Dedicated Hosting

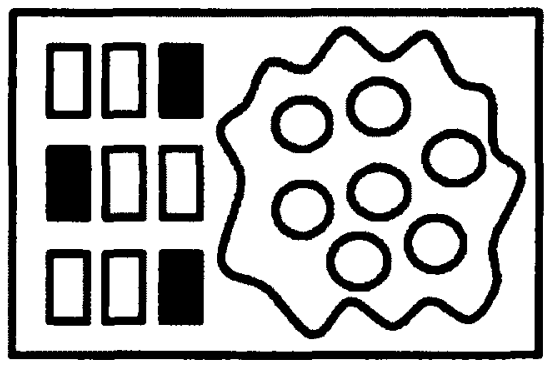

d) Cloud Hosting

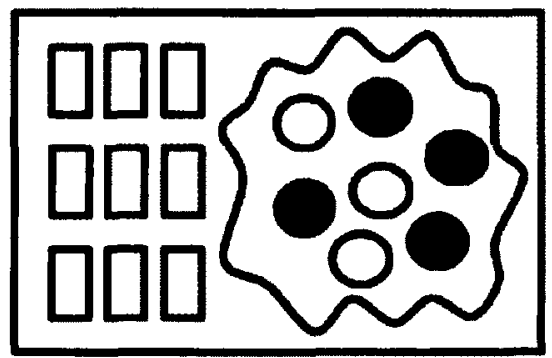

Virtual Server (employed)

Virtual Server (not in use)

Figure 2-2: Infrastructure Hosting Types (based on [BAR-12])

\subsubsection{Dedicated Hosting}

Dedicated hosting, demonstrated in Figure 2-2b, is where a client rents a number of dedicated physical servers from anywhere inside the data centre on-demand. Although the hardware used is dispersed throughout the data centre with the other customer's hardware, the physical servers utilized are not shared with anyone else. Dedicated hosting is less expensive than private cloud hosting and because it is on-demand it provides the client with the ability to increase or decrease the number of servers being used [BAR-12].

\subsubsection{Hybrid Hosting}

Displayed in Figure 2-2c, hybrid hosting refers to when a client rents a combination of both dedicated physical servers and virtual server instances which are less expensive on 
demand [BAR-12]. Like dedicated hosting this allows the client to scale up or down the number of physical and virtual servers used as required.

\subsubsection{Cloud Hosting}

Cloud hosting pertains to when a client rents a number of virtual server instances on demand and thus share all of the servers with other customers [BAR-12] (refer to Figure 2-2d). As a result of this, cloud hosting is the least secure form of hosting. On the other hand, cloud hosting is the cheapest and the most technically efficient and environmentally friendly form of hosting as it provides the provider with the opportunity to operate their physical servers being used to capacity and to shut down the ones that are not needed.

\subsubsection{Deployment Models}

This section describes the four deployment models known as private cloud, community cloud, hybrid cloud, and public cloud.

\subsubsection{Private Cloud}

The private cloud provisions the infrastructure for restricted use by a sole organization [MEL1-11]. Users of the private cloud do not benefit from the lower capital costs as they are still required to purchase, build and manage the hardware and software [MAL-11]. Since only the organization's users are granted access to the private cloud, security is enhanced [JAD-12]. 


\subsubsection{Public Cloud}

The public cloud is made available to the general public via a service provider on a payper-use basis [MAL-11]. Typically these service providers own and operate the infrastructure and grant access only through the internet.

\subsubsection{Community Cloud}

The community cloud shares infrastructure between multiple organizations from a specific community sharing the same concerns such as mission, security, and compliance considerations [MEL1-11]. The infrastructure may be hosted and managed internally or by a third-party [JAD-12]. Since the costs are spread over a lower number of users in comparison to a public cloud, only some of the cost savings potential of cloud computing are realised.

\subsubsection{Hybrid Cloud}

The term hybrid cloud is used to represent a combination of two or more clouds (ie. private, public or community) which remain as exclusive entities but are combined providing the benefits of multiple deployment models [MEL1-11]. Hybrid cloud architectures require both on-site and off-site resources, and lack the flexibility and security of in-house applications [OXY-12].

\subsection{Grid and Cloud Components}

This section describes the common core components used in grids and clouds.

\subsubsection{Security}

Security is an important aspect of both grids and clouds. Since these systems provide users access to resources which may contain sensitive information, it is imperative that 
there are mechanisms in place to provide security, including authorization, authentication, and data encryption [JAC-03]. The security component ensures that an appropriate level of protection is maintained for all users in the system. The Grid Security Infrastructure (GSI) is the component within the Globus Toolkit which is responsible for security [FOS-01]. The Amazon EC2 Security component provides the required security services within the Amazon EC2 cloud [KRE-10].

\subsubsection{Matchmaking}

Matchmaking refers to the process of selecting a single resource or single resource set from a set of available resources that meet the minimum requirements of the request [JAC-03]. This component is commonly referred to as the broker and its main purpose is to enhance system performance and efficiency. Different criteria can be employed by the broker for attaining high system performance. For example, incoming requests may be matched to resources such that the response time is minimized.

\subsubsection{Scheduling}

Scheduling deals with the order that requests are to be run on a resource. The number of requests that can be scheduled at a given time is impacted by the ordering of the requests. There can be different levels of schedulers within a system [JAC-03]. In systems that contain clusters of resources, a high level scheduler, known as a meta-scheduler, may be used to schedule work done on the cluster, while the scheduler on the cluster would take care of the actual scheduling of work on the cluster's individual nodes. The AppLeS (Application Level Scheduling) Parameter Sweep Template, discussed in [CAS-00] is an example of a commercially available scheduler component that can be employed in a grid and cloud environment. 


\subsubsection{Resource Management}

In grids and clouds resource management is a challenging task due to the dynamic sharing of heterogeneous resources where these resources may belong to different administrative domains and may span over a large geographical area. These resources can be added or removed from the system at any time. A resource management component is required so that these resources are managed effectively. Resource management consists of 4 roles, including resource discovery, matchmaking, scheduling, and resource monitoring [YUA-11]. One example of the resource management component for grids is the Grid Resource Allocation Manager (GRAM) existing within the Globus Toolkit [BER-02]. Related to clouds, an example of this component is Haizea which is an open source resource lease manager [AKH-11].

\subsubsection{Data Virtualization and Data Management}

As requests are executed, data may need to be made available to the node that is executing the request. Since grid and cloud systems contain geographically distributed resources, data and computing power may be distributed as well. It is imperative that access and management of distributed data be simple and effective, as if the data was stored in a central server [JAC-03]. This component provides these data related services. Within the Globus Toolkit, this component is known as the Grid Access to Secondary Storage (GASS) [BER-02].

\subsubsection{Charge-back}

Businesses use charge-back to track the user's access of computing and data resources for the purpose of charging users for using the system's resources. Utility grids and commercial clouds commonly use charge-back. Some examples of these systems that use 
charge-back are Amazon's S3 which provides a data storage service [AMA-12], Amazon's EC2 which offers users with computational resources [AMA-12], and Google's App Engine [PRO-12] which provides web-application developers with resources to build and run their applications.

\subsection{Grid and Cloud Workloads}

Workloads consist of tasks or requests that are executed on grid and cloud resources for applications. In the scope of this thesis, the terms tasks, requests and jobs are interchangeable and meant to refer to the same entity. This section discusses the different types of requests typically found in a grid or cloud, and the workload parameters associated with them.

\subsubsection{Request Types}

- On-Demand (OD) requests are the most common type of request received in grid and cloud environments. Most systems place OD requests in a waiting queue if no resources are available [SUL-04]. Because of this, these types of requests are served on a best-effort basis.

- Advance Reservation (AR) requests are suited for applications that commence execution at some point in the future. An Advanced Reservation request contains all of the necessary information about the application for the resource to be reserved. ARs are used for applications that demand QoS guarantees.

- Emergency (E) requests are extremely rare and only occur in emergency situations. Emergencies have the highest priority level in the system. They begin execution immediately provided another Emergency request is not already running and release the resource when it has completed. 


\subsubsection{Workload Parameters}

This section discusses the workload characteristics used in defining the different requests. For $\mathrm{E}$ and $\mathrm{OD}$ requests, the service time is enough to sufficiently define it.

For AR requests, every AR has an earliest start time which depicts the earliest time that the request can begin executing. This is beneficial in circumstances where the request requires data that is only obtainable at some time in the future. The request is associated with a deadline which signifies the latest time that the work to be executed must be completed. The service time of the request represents the amount of time required to execute its job. AR requests also have an extra window of time called laxity. As a result of this characteristic every request has a start time which denotes the time that the request is scheduled to commence execution. This may or may not be the same as the earliest start time. The symbols used to portray these characteristics are:

EST: Earliest Start Time

$S:$ Service Time

$L$ : Laxity

$D$ : Deadline, given as $D=E S T+S+L$

\section{$T:$ Start Time}

An OD request that is not associated with a deadline can be considered as a special case of an AR request with an arbitrarily large deadline. 


\subsection{Related Work on Resource Management}

This section describes the related work associated with matchmaking, scheduling and advance reservations in grid and cloud systems. In Section 2.5.1 matchmaking and scheduling algorithms that are not concerned with user estimates of request runtimes are presented. Section 2.5.2 provides various methods used in grids and clouds to handle advance reservations and Section 2.5.3 discusses techniques associated with handling ARs with priorities.

\subsubsection{Matchmaking and Scheduling in Grids and Clouds}

Grid and cloud computing are prospering technologies that offer users a way of solving complex problems by submitting their computing tasks to the system. Since grid and cloud resources are restricted by their physical capacities, as the demands of these resources by the user increase, matchmaking and scheduling play a significant role to ensure these resources are used effectively [SOT-11]. Several matchmaking and scheduling strategies have been discussed in the literature. This section presents a representative set of them.

In [LI-11] the authors present a matchmaking algorithm derived from the Ant Colony Optimization (ACO) algorithm [DOR-06]. ACO is based on the behaviour of real ants that have the ability of finding an optimal path from nest to food. The proposed algorithm called Load Balancing Ant Colony Optimization (LBACO) inherits the fundamental ideas from the ACO algorithm to allocate tasks so that response times are reduced while achieving a well-balanced load across all virtual machines (VMs) in the cloud. In LBACO, a virtual pheromone value which decays over time is assigned to every VM. When a task must be allocated, ants are placed at the starting VMs randomly. Every ant 
travels to other VMs randomly and attempts to select a VM that can serve the task. The higher the pheromone value of the VM, the higher the probability of the ant choosing that VM for the task. The pheromone value of the VM is high if the VM has a light load and has enough speed to serve the task. When an ant locates an acceptable VM for the task it intensifies the pheromone value of all the VMs that it has traveled to. Also, if the path traveled by the ant is the shortest path amongst other ant paths, all VMs in that path are augmented with higher pheromone values. The higher pheromone values of the VMs increase the probability that these VMs are considered for future tasks. The performance of the LBACO algorithm is examined using simulation where the results show that it outperforms other matchmaking strategies such as the basic ACO and First-Come-FirstServed (FCFS) algorithms.

In [MOS-12] the authors provide an evaluation of the performance of the most commonly used strategies around gang scheduling policies. Gang scheduling is a special case of scheduling parallel jobs in which the tasks of the jobs need to communicate often. The policies are simulated in a cloud system equipped with dynamic provisioning of VMs pertaining to the system's current load. Every VM in the system maintains its own queue for the tasks of the parallel jobs waiting to be executed on that VM. The entry point for an arrival is the Dispatcher Virtual Machine (DVM) which dispatches the job's tasks to the VMs provided that the number of tasks is less than or equal to the system's available VMs. The DVM also has a waiting queue for jobs that are unable to be dispatched when they arrive either because of overloaded VMs or inadequacy of VMs. DVM uses the Shortest Queue First (SQF) algorithm to allocate tasks to the VMs. SQF dispatches tasks to VMs with the least loaded queues where tasks that belong to the same job cannot 
reside in the same queue as this would violate the one-to-one mapping of tasks to VMs that is required by gang scheduling.

Two gang scheduling policies for scheduling tasks are used in the study including Adaptive-First-Come-First-Served (AFCFS) and Largest-Job-First-Served (LJFS). In the first algorithm, AFCFS, every time a VM becomes idle, it searches through a list of jobs that are ready to run to find a suitable job for execution. In order for a job to be chosen for execution, all of its tasks need to be at the front of the VM's waiting queue, and all of these VMs must be idle as well. If no job like this is available, AFCFS attempts to schedule jobs whose tasks are further back in their queues in the same manner until it either comes across such a job, or reaches the end of the queue. Hence, this approach of scheduling jobs tends to favour jobs that require a smaller number of VMs. In the second algorithm, LJFS, jobs are scheduled according to their size, which is represented by the number of tasks belonging to that job. Higher priority is given to jobs with larger sizes. A list of ready to run jobs are ordered by size in descending order and is maintained by the system. When a VM becomes idle, the system goes through this list to determine which job to execute. The first job whose tasks are allocated to idle VMs is scheduled for execution. The simulation results of these gang scheduling policies examined in [MOS12] show that they can be efficiently applied to a cloud system with dynamic provisioning of VMs.

In [ELM-08] a grid resource broker that supports Advance Reservation requests is presented where the broker performs matchmaking based on criteria set by the user. The main objective of the broker is to minimize the response time of the requests which encompasses the time required for submission of an input file and executables to the 
resource, time waiting in the batch queue, execution time, and the time for transferring the output files to the specified location. The best resource for an incoming request is determined by the user selecting one or more bench marks with performance characteristics similar to the request. Since both the bench marks and the requests have similar computation requirements, the bench marks are used to predict the execution time of the request. The network performance is predicted using the Weather Network Service (WNS) [WOL-99] where the periodic bandwidth measurements are combined with the statistical methods to make short term predictions about the available bandwidth. The combination of the predicted network performance along with the predicted response time using the bench marks is used to determine the resource mapping leading to the minimum execution time of the request. The broker also has the ability to reallocate a reservation to another resource if it is determined that another resource can reduce the response time.

In [MAJ-09], the author introduces the Any-Schedulability (AS) criterion, which allows the matchmaker to determine if a set of AR requests can meet their deadlines at the resource without knowing the local scheduling policy. The only condition required by the criteria is that the local scheduling policy be work-conserving. A work-conserving scheduling policy is achieved when the CPU is never kept idle when a request is available for execution. The AS theorem states that a set of AR requests (where $\mathrm{N}$ denotes the size of the set) will be successfully scheduled if the specific set of inequalities mentioned in [MAJ-09] are satisfied for each request $i(\mathrm{i}=1 . . \mathrm{N})$. 


\subsubsection{Advanced Reservations}

As mentioned in Section 2.4.1 applications can submit requests in two different ways, either by submitting them to the resource queues that are served on a best-effort basis or by reserving the resources at a specific time in the future. Advanced Reservation (AR) requests are suited for applications that commence execution at a time in the future. They contain details about the application including the earliest start time, the deadline, and the estimated duration of execution. The advanced reservation is defined as the process of obtaining a resource's capability for a specified time interval through negotiations with the resource provider to certify the guaranteed availability of the resource [DIS-08]. This section discusses some relevant research performed in the area of using AR requests to provide QoS guarantees to consumers.

AR requests originated as part of the Globus Architecture for Reservation and Allocation (GARA) for two main reasons [FOS-99]. First, they allow multiple jobs of an application to be co-allocated on different resources simultaneously. Secondly, it guarantees the resource allocation for grid applications. Since the introduction of ARs, they have been used in several other contexts including grid based architectures for dynamic optical networks [LAV-04] and architectures for data-intensive collaboration [FOS-03]. The performance of advance reservation based scheduling has been investigated in [SUL-04].

\subsubsection{Overestimation of Execution Time}

Users are required to provide estimates for execution time that the request will run on the resource when dealing with ARs. Schedulers use the estimated execution time to order the requests as they run on the resources. Users tend to overestimate the execution time. An explanation for this is that users overestimate the request's execution time to prevent 
their requests from running out of time and being aborted [SUL-04]. The overestimation is typically large. In [BAI-05] the authors state that on average the majority of requests use less than $20 \%$ of time they requested. In some extreme cases, according to [FAR-07], users can overestimate the execution time by as much as $25,000 \%$.

A relaxed Advance Reservation policy (RARP) which allows reservations to overlap with one another under certain conditions is proposed in [RAN-11]. The objective of RARP is to increase the resource utilization rate and reduce the rejection rate when the request deadlines have been overestimated. The focus is on AR requests without laxity. Requests that are scheduled are given a distinct time slot. As a result of overlapping the requests, the reserved time slot is no longer reserved for a particular request but can be provisionally allotted to multiple requests. Since the authors expect that the execution time associated with the request specified by the user is often overestimated, the reservation violation previously mentioned is allowed. An attempt at predicting the overestimation of the request's execution time specified by the user is made to get the true value of the execution time which is smaller than the overall request execution time. This allows for more requests to be scheduled. If the prediction technique is not properly tuned this strategy has a risk of performing reservation violation where reservations are not able to meet request deadlines.

In [BIR-09] the authors use a similar strategy by attempting to resolve the problem of overestimation using overbooking by taking the probability of resources failing into consideration. Overbooking means placing a request in a gap of a schedule that is smaller than request's maximum execution time (ie. overlapping of requests). Requests are overlapped according to the probability of success (PoS). This is computed using the 
probability of a machine failure and the probability that the incoming request finishes on time. The PoS is calculated for each free space detected between the scheduled requests where the incoming request might be scheduled. The overbooking algorithm can use PoS in several ways. One approach is that it can place an incoming request in the gap with the highest PoS. Another is a first fit approach which places the incoming request in the first gap with an acceptable PoS.

In [FAR-09] the authors showed that the overestimation error in a request's estimated runtime in the real workloads collected from the IBM SP2 supercomputer is extremely large. Since the feasibility analysis at the time of an incoming request relies on an estimation of the request runtime provided by the user, extremely large overestimation of request runtimes lead to large amount of unnecessary rejections. Because of this, performance is severely reduced. In order to tackle the problem associated with the inaccuracies of user estimated runtimes, the authors used a pre-scheduling mechanism, which permits overbooking of the system with requests. The system has an overbooking threshold which allows a small proportion of requests to miss their deadlines. When a request arrives, the system performs a schedulability analysis. If it is determined that accepting the request does not result in the proportion of requests that miss their deadlines to be greater than the overbooking threshold, the request can be accepted by the system. Since the majority of requests have overestimated runtimes, the actual number of requests that miss their deadline is lower than the threshold. By allowing a small proportion of requests to miss their deadline, the authors state that the utilization of the system is improved. 


\subsubsection{Frameworks for Management of Advance Reservation Requests}

A generic framework that allows grid schedulers to manage ARs across heterogeneous local Resource Management Systems (RMS) is proposed in [QU-07]. Presently, there is a limited number of RMSs that are capable of supporting ARs including Platform LSF [PLA-12]. As well, most of the current grid schedulers such as LSF Multi-Cluster [PLA12] are only capable of managing $A R$ requests within a homogeneous environment, where all of the RMSs are of the same type and are AR-capable. The proposed framework enables AR functionalities regardless of whether the local RMSs can handle ARs or not. The framework's key component is the AR manager which is able to externalize the AR functionality from local RMSs, and further interact with local systems. In order to externalize the AR functionality from the local RMSs, the AR manager uses an AR algorithm and a list of ARs for each local RMS. The AR algorithm permits the AR manager to schedule requests to local RMSs for local scheduling, or in the case where the RMS does not support ARs, the AR manager schedules the AR directly on the resource. In order to use the framework, two sets of APIs are used. One implemented at the local RMSs which allows communication between the AR manager and the local RMSs, and another implemented at the AR manager to expose full sets of AR functionalities.

In [TAK-08] the authors present a Grid Advance Reservation-based System (GridARS) framework used for Advance Reservations-based co-allocation within grids. GridARS is able to co-allocate distributed resources managed by various local schedulers by using a global resource scheduler in combination with the local resource schedulers. The GridARS framework addresses several issues including co-allocation of various types of 
resources, performing resource management by coordinating the global scheduler and the local schedulers, providing an advance reservation capability for local and global schedulers to co-allocate resources, an interface for global users for submitting requests with secure communication, and a two-phase commit protocol for requesting and committing reservations. These issues are resolved by combining a global scheduler with resource managers (RM) for computers, networks and other resources. In each RM, a resource scheduler manages a reservation table of its resources for advance reservation. When a user sends requirements on resources and reservation time to the global scheduler, the global scheduler uses the reservation table of the resource manager to reserve the resource and schedule the request.

The authors in [TAK-11] extend the GridARS framework for the Intercloud. The Intercloud is a virtual infrastructure distributed geographically, and over multiple domain clouds that performs large-scale data intensive scientific computing and avoids cloud lock-in. Here GridARS can integrate heterogeneous resources and construct a performance-assured virtual infrastructure over an Intercloud environment. The critical issues faced for providing a performance-assured virtual infrastructure in an Intercloud environment include coordination of various resources from multiple heterogeneous domains, creating a suitable resource allocation plan that reflects reservation options for users, providing applications with a virtual flat environment, and monitoring the usage of distributed virtual resources managed by multiple domains. To solve these issues GridARS provides four service components including the resource management service, the resource allocation planning service, the application execution management service, and the distributed monitoring service. 


\subsubsection{Advance Reservations with Priorities}

In [MIN-01] the author presents a novel non-pre-emptive scheduling algorithm called Reservation Scheduler with Priorities and Benefit Functions (RSPB) which schedules ARs while considering the priorities of the requests. Every request is associated with a start time, end time, priority, minimum CPU requirement for delivering the maximum benefit to the application, and benefit function for quantifying the benefit that the user would acquire by securing the resource at the specified priority level. The benefit function removes the need for negotiations when there is resource scarcity. The objective of the scheduler when the overall demand exceeds the available resources is minimizing the sum of priority of the requests that are rejected. In RSPB the scheduler makes decisions after the arrival of a batch of requests which forms a meta-request $\left(\mathbf{R}_{\text {meta }}\right)$ with a predefined time interval. The requests within $R_{\text {meta }}$ are sorted in descending order by priority level which ensures that if multiple requests require reservation at the same time, the requests with higher priority will be scheduled first. A machine may be selected if the hard QoS requirement is met. If none of the machines can satisfy the hard QoS requirement, the RSPB will attempt to schedule the request with degradation of the CPU requirements provided the benefit function allows this. A machine is selected by providing the maximum benefit for the application by the reservation. The RSPB algorithm is compared with another AR algorithm called the Resource Broker (RB) developed [KIM-00]. The simulation results indicate that the number of rejections is considerably lower for RSPB than RB.

The work presented in [AHU-09] proposes a dynamic priority scheduling system for advance reservation consisting of two components for handling priorities and protocols of 
the jobs dynamically without considering pre-emption. The components are referred to as Dynamic Priority Scheduler for Advance Reservation (DPSAR) which schedules prioritized jobs and submits them to the Advance Reservation Module (ARM) which allocates resources to the reserved jobs at a specific time in the future. In this strategy, both the users and their jobs have an associated priority. After users have joined the system, DPSAR goes through four stages. In the first stage users are placed in a queue that is sorted in descending order of their priorities. In the second stage a gridlet sorting policy (GSP) is used to sort the jobs of each user. The GSP sorts the jobs of each user based on a shortest job first technique. In the third stage the jobs are further prioritized using a Dynamic Priority Resolution (DPR) technique which sorts the jobs of the various users in accordance with the job's priority based on job length, and on the user's priority to which the jobs belong to. GSP also decides how many jobs of a high priority user are to be considered for reservation. This prevents all of the jobs of a high priority user from being entertained which avoids starvation of jobs of low priority users. In the last stage, the prioritized jobs are placed in a queue and sent to ARM for allocation. The DPSAR has been compared with another algorithm called Advance Reservation Without Priority (ARWP). The simulation results show an improved performance of DPSAR over ARWP in regards to resource utilization and the number of rejections.

A number of techniques for handling advance reservations in grid and cloud systems have been discussed in this chapter. This thesis investigates a pre-emptive approach for handling advance reservations with priorities. To the best of our knowledge, none of the existing studies presented a solution for dealing with advance reservations where higher priority ARs have the ability to pre-empt lower priority ARs that have already been 
reserved. In addition, this thesis also studies the effects of Emergency requests in such a system. To the best of our knowledge, no other work has addressed the issue of handling Emergency requests in the context of ARs with priorities. Along with the Emergency request concept, Chapter 3 describes the simulation model of the system including the various matchmaking and scheduling policies proposed for handling emergencies and advance reservations with priorities. 


\section{Chapter 3 - Resource Management Techniques}

This chapter concentrates on resource management techniques for systems that are subjected to Advanced Reservation requests with priorities and Emergency requests. More specifically it describes the various matchmaking strategies used throughout this thesis.

\subsection{Matchmaking Based on Knowledge of Scheduling Policies}

The purpose of this matchmaking strategy considered in this thesis is to select resources for executing Advanced Reservation and Emergency requests without using pre-emption or abortion techniques. The broker uses knowledge of the scheduling policy employed at a given resource to determine if the newly arriving request can meet its deadline when allocated on this resource. The resource then manages the scheduling of the new request pertaining to the scheduling policy. When dealing with a system with multiple resources, the request is allocated to a resource from the broker by using the allocation algorithms. If a request is rejected by all of the resources it is deemed unschedulable and rejected by the system if the incoming request has a priority level of 0 . If the incoming request has a priority level greater than 0 , the broker will perform a further analysis for abortion or preemption as outlined in Section 3.2 and Section 3.3 respectively in search of finding a resource that can accommodate the request.

\subsubsection{Allocation Algorithms}

The broker uses the matchmaking strategy based on the knowledge of the local scheduling policies by carrying out the allocation algorithms. The algorithms commence with the broker requesting a list of available resources from the Grid Information Service 
(GIS). Once the resource discovery process is complete, the broker requests the resource characteristics containing all of the current requests allocated on the resource. With this knowledge and that of the resource's scheduling policy the broker then simulates the resource's scheduling policy to determine if the incoming request is schedulable at that resource. If the request is schedulable on multiple resources, the First Fit and Next Fit allocation algorithms are used to select the resource that the request is allocated on. If the request is not schedulable on any of the available resource, the request is rejected if its priority level is 0 , otherwise the broker will perform additional matchmaking strategies outlined in Section 3.2 and Section 3.3 .

\subsubsection{First Fit Allocation Algorithm (FFAA)}

In this allocation algorithm the broker selects the first available resource starting from the first resource in the resource list where the request is schedulable without pre-empting or aborting another request [FAR-07].

\subsubsection{Next Fit Allocation Algorithm (NFAA)}

In this allocation algorithm the broker keeps track of the last resource from the resource list that allocated a request. The broker selects the first available resource from the resource list where the request is schedulable starting with the resource that appears directly after the last resource from the resource list that successfully allocated a request without pre-empting or aborting another request [FAR-07]. 


\subsubsection{Scheduling Policies}

The following section provides a description of the scheduling policy used by the resources and the broker for new arrivals to determine if the incoming request is schedulable on a specific resource.

\subsubsection{Earllest Deadline First Scheduling}

The Earliest Deadline First scheduling policy, abbreviated as EDF, uses the deadline of the request to determine the order that it will be scheduled [MEL-10]. A request with an earlier deadline is allowed to execute earlier than requests having later deadlines. When an incoming request arrives at the broker, the broker determines if the request is schedulable on a given resource based on its deadline. This scheduling policy is non-preemptive. The scheduler may postpone another request with a later deadline but will not pre-empt a request to accommodate it. The request is rejected if it is deemed not schedulable by the broker. In order to make this scheduling policy abide by the work conserving principle, this policy has been modified [MEL-10]. The work conserving principle states that the CPU is to never be left idle when a request is available for execution. This is accomplished by scheduling a request during a free idle time in the resource's schedule even if its deadline is not the earliest among the already scheduled requests. If there is no resource idle time slot available the request is scheduled according to its deadline.

\subsection{Handling Advanced Reservation Requests with Priorities}

This section describes the matchmaking strategies for handling Advance Reservation requests with various priority levels. 


\subsubsection{Advance Reservation Priority Level}

This thesis investigates the performance of a pre-emptive system containing Advance Reservation requests with priorities. Therefore, the AR requests used in this research have an associated priority level indicating the scheduling priority for requests when they are not accepted using the allocation algorithm and is represented by the symbol $p$. If the request is accepted using the allocation algorithm, the scheduling priority follows EDF as described in Section 3.1.2.1. The higher the importance of the request, the higher the priority level associated with that request is. In this research a request can have a priority level $p$, from 0 up to 2 . Incoming requests can only abort lower priority requests that are already scheduled. For the reason mentioned in Section 4.5.2.7, this thesis considers AR requests with two different priority levels; low priority $A R$ requests $\left(A_{L}\right)$ which have a priority level set to 0 , and high priority $A R$ requests $\left(\mathrm{AR}_{\mathrm{H}}\right)$ which have a priority level set to 1 . Emergency requests have the highest priority out of all requests, and are thus modelled with a priority level of 2 .

Since ARs provide a QoS guarantee that once scheduled on a resource they will complete their execution by their deadline, this thesis restricts AR requests from pre-empting other ARs currently executing regardless of the priority level. However, this research does permit high priority AR requests with the ability to abort low priority ARs that are scheduled to be executed in the future, where the aborted low priority ARs have the ability to be reallocated on another resource provided there is a spot for them. If the low priority AR cannot be reallocated on another resource, it is aborted from the system. Emergency requests have the ability to pre-empt any running task except for other emergencies. 


\subsubsection{Matchmaking Based on AR Request Priority Level}

The purpose of aborting requests investigated in this thesis is to accommodate ARs with a priority level greater than 0 when these requests cannot be allocated using the matchmaking strategy based on knowledge of the scheduling policy described in Section 3.1. An AR request is aborted from the system when it is removed from a resource after being scheduled in order to accommodate a higher priority request and is no longer able to meet its deadline on any of the other available resources. The aborted request may have been pre-empted or aborted from running in the future. Pre-emption is the act of temporarily internupting an AR that is executing, without its cooperation, and with the intent of resuming the $\mathrm{AR}$ at a later time to accommodate a request with a higher priority. In this research only Emergency requests have the ability to pre-empt AR requests. An AR does not have the ability to pre-empt another ARs regardless of priority level for a few reasons. First, ARs provide a QoS guarantee to meet their deadlines once accepted, and allowing ARs to pre-empt other ARs with a lower priority level would violate this QoS guarantee. Second, AR requests regardless of priority are not always accepted, whereas Emergency requests are always accepted and pre-emption is mandatory. Third, this research is concerned with Advance Reservations where pre-emption may be necessary for accommodating emergencies only. For these reasons, the abortion policies presented in this thesis used for accommodating high priority AR requests do not consider pre-emption. Refer to Section 3.3 for a discussion on how the system handles Emergency requests and the pre-emption policies that are used. Although this thesis does not permit ARs to pre-empt other ARs currently executing regardless of priority level, the abortion policies described in Section 3.2.3 allow the ability for ARs to abort lower 
priority ARs that are scheduled to run in the future. The reason for this is that an aborted request has a better chance of being reallocated on another resource than a pre-empted request because the aborted request has a deadline which is further in the future allowing a larger window of time to be reallocated.

When the broker receives a request from the source, it first performs the matchmaking strategy based on the knowledge of the scheduling policies outlined in Section 3.1. Here, the broker determines if the request is schedulable on one of the available resources by using the allocation algorithms for scheduling the request without aborting other scheduled jobs. If a low priority AR request is rejected by all of the available resources, it is considered unschedulable and rejected by the system. When the broker determines that a high priority AR request is rejected by all of the resources using the allocation algorithm, the broker uses the abortion policies in another attempt to accommodate the request.

\subsubsection{Abortion Policies}

As mentioned in Section 3.2.2, in reference to this research the term abortion refers to the act of removing a job that has not yet started execution, but has been scheduled to run in the future to accommodate a higher priority AR request. The abortion policies proposed in this thesis consider only Advance Reservation requests with multiple priority levels commencing in the future, and do not include Emergency requests as emergencies begin immediately and require pre-emption. Refer to Section 3.3 for how the system manages Emergency requests. The abortion policies are used by the broker for matchmaking AR requests with priority levels greater than 0 that were not allocated using the matchmaking strategy based on the knowledge of the scheduling policy outlined in Section 3.1. 
Provided that the broker finds a resource to accommodate the incoming request pertaining to the given abortion policy, it also selects the time the request will begin execution, again based on the policy used. After determining the appropriate resource and the time for starting the incoming request, the broker sends a message to this resource to remove the respective conflicting job(s) and has it/them sent back to the broker in an attempt to reallocate it/them on another resource. After the conflicting job(s) has/have been removed, the broker sends the incoming request to the chosen resource for allocation. When high priority $\mathrm{AR}$ requests are accommodated by aborting (replacing) existing lower priority AR requests, the order of the requests may not follow EDF anymore. The conflicting job(s) that was/were sent back to the broker to be reallocated are processed by the matchmaking strategy based on the knowledge of the scheduling policy. Note that the priority level of the conflicting job remains unchanged when sent back to the broker. If the conflicting job(s) cannot be rescheduled on any of the other resources, it/they is/are aborted from the system. The following sections describe the abortion policies proposed in this research. Each abortion policy is based on a characteristic of the workload and/or system. One of the motivations behind this research is to investigate and compare the effectiveness of using each of these characteristics in matchmaking.

\subsubsection{Minimum Conflicting Jobs Algorithm (MCJA)}

In order to explain this algorithm, a few terms are now defined:

Execution Window (EW) - The execution window of an incoming request is the time interval during which the request can be executing. The EW begins at the request's earliest start time and ends at its deadline. 
Conflict Window (CW) - The conflict window is a window of time that moves through the incoming request's EW and is used to determine the number of conflicting jobs (ie. scheduled jobs with a lower priority level) that exist for the incoming request at a given start time for the $\mathrm{CW}$. The conflict window is of the same duration as the request's service time. The purpose of the moveable $\mathrm{CW}$ is to determine the start time(s) of when the incoming request is to be scheduled.

Conflicting Job - A conflicting job is a job that is currently scheduled to run on the resource within the incoming request's conflict window for a given $\mathrm{CW}$ start time. Only the conflicting job(s) with an associated priority level that is/are lower than the incoming request is/are considered for abortion.

This algorithm which is used by the abortion policies concerns the schedulability of the incoming high priority AR requests on a given resource by aborting lower priority AR requests. It analyses the $\mathrm{EW}$ of the incoming request. By sliding the $\mathrm{CW}$ through the $\mathrm{EW}$, this algorithm is able to determine the minimum number of conflicting jobs for a given resource. The $\mathrm{CW}$ advances to the start times and expected finish times of all conflicting jobs within the EW and the algorithm analyses the conflicting jobs at each of these CWs until $\mathrm{CW}$ reaches the end of the EW. The algorithm then returns one or more start times used for scheduling the incoming AR request. These start times are the same as the start times of the CWs that achieve the minimum number of conflicting jobs in the EW on the given resource. These start times for scheduling the incoming request are then used by the abortion policies described in the following sections for selecting the conflicting low priority AR request(s) to be aborted. In the event that no start time is returned, it implies that the incoming request cannot be accommodated on the given resource. 
An example of how MCJA uses the conflict and execution windows is shown in Figure 3-1. The incoming high priority AR request to be allocated is characterized by an earliest start time, EST, a service time, S, and a deadline, D. The starting and finishing positions of the incoming request's conflict window, $\mathrm{CW}$, are indicated in the figure. The $\mathrm{CW}$ slides through the execution window, EW, from left to right starting at the incoming request's EST and ending at its D. In the figure, the $\mathrm{CW}$ at the starting position identifies two conflicting low priority $A R$ requests, $A R_{L-1}$ and $A R_{L-2}$. Since both of these conflicting jobs have a lower priority than the incoming request, they are considered for abortion. The $\mathrm{CW}$ at the ending position identifies one conflicting high priority $\mathrm{AR}$ request, $\mathrm{AR}_{\mathrm{H}-1 .}$. This conflicting job is not considered for abortion because the incoming high priority AR request cannot abort an existing high priority request. In this example, for the given resource the minimum number of conflicting jobs is $2, A R_{L-1}$ and $A R_{L-2}$, and MCJA returns the time indicated by EST as a possible start time for when the incoming request can be scheduled.

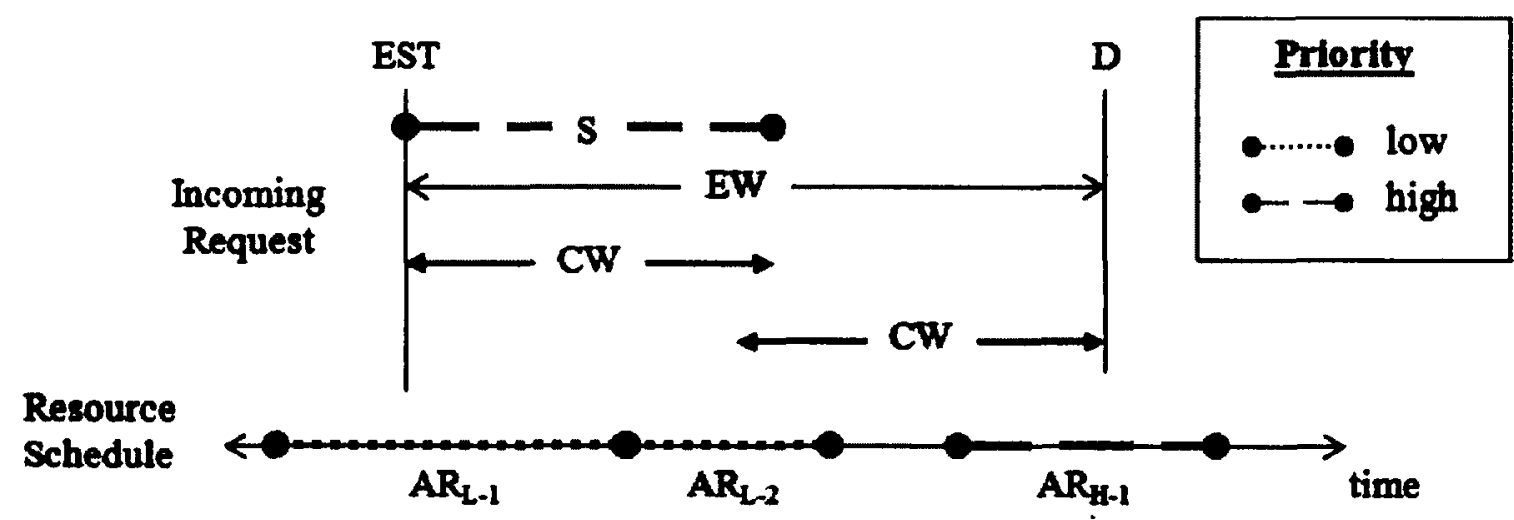

Figure 3-1: MCJA Example 


\subsubsection{Next Resource Abortion Policy (NR-A)}

The broker considers only the resource that is next to the one that was used for performing the last abortion in the resource list. MCJA is used by the broker to determine whether this resource can accommodate the incoming high priority request. If not, the incoming request is rejected from the system. If the incoming request can be accommodated, the broker schedules the request at the earliest start time returned by MCJA on the resource. The conflicting job(s) is/are aborted and sent back to the broker for reallocation.

\subsubsection{Next Abortable Resource Abortion Policy (NA-A)}

Like NR-A, the broker keeps track of the resource from the resource list that last aborted a job $\left(R_{L A}\right)$. The difference between NR-A and NA-A is that NR-A only checks the resource that appears directly after $R_{\mathrm{LA}}$ in the resource list. NA-A on the other hand uses MCJA on all the resources in the resource list starting from the next resource after $R_{L A}$ until it finds a resource to accommodate the high priority AR request. If none of the resources can accommodate the incoming request, the request is rejected from the system. If the incoming request is accommodated on a resource, the conflicting job(s) is/are aborted and sent back to the broker for reallocation.

As opposed to NR-A and NA-A, in the remaining abortion policies (described in Section 3.2.3.4 and Section 3.2.3.5) the broker analyses all of the available resources with MCJA to produce a resource candidate set which consists of the resources that achieve the minimum number of conflicting jobs. An empty resource candidate set indicates that none of the available resources can accommodate the incoming request. If the resource candidate set is empty the incoming request is rejected from the system and no 
conflicting jobs are aborted. For the remaining policies, the broker iterates through the resource candidate set analysing the resources based on a specific characteristic used in the abortion policy to determine the most appropriate resource on which the request is to be allocated. The time at which the incoming request is to be scheduled is also determined by the abortion policy. The following sections describe the remaining abortion policies in detail.

\subsubsection{Largest Sum of Slack Times Abortion Policy (LS-A)}

The term slack is the amount of time between when the conflicting job is expected to finish and its deadline (ie. slack = D - EFT). This policy computes the sum of the slack times of the conflicting jobs for each of the CWs that correspond to the start times which are returned by MCJA for all of the resources in the resource candidate set. Note that for a resource with multiple sets of conflicting jobs, each of which corresponds to a specific $\mathrm{CW}$, multiple sums of slack times are computed. The broker selects the resource from the resource candidate set with the largest sum of the slack times. The incoming request is then scheduled on this resource at the start time of the $\mathrm{CW}$ that results in the largest sum of the slack times. Ties are broken in favour of the first resource the broker analyses from the resource candidate set containing the largest sum of the slack times. The motivation behind this policy is that aborting the conflicting job(s) with the largest sum of the slack times will allow a better chance for the conflicting job(s) to be scheduled on another resource.

\subsubsection{Greatest Sum of Service Times Abortion Policy (GS-A)}

This policy computes the sum of the service times of the conflicting jobs for each of the CWs that correspond to the start times which are returned by MCJA for all of the 
resources in the resource candidate set. The broker selects the resource from the resource candidate set with the greatest sum of service times. The incoming request is then scheduled on this resource at the start time of the $\mathrm{CW}$ that results in the greatest sum of service times. Ties are broken in the same way as in the case of LS-A described in Section 3.2.3.4. The motivation behind this policy is that aborting the conflicting job(s) with the greatest sum of service times will minimize the number of requests that are aborted because of high priority ARs.

Other abortion policies similar to LS-A using different metrics have been simulated. However, they are not presented as they did not produce significantly different results. These other policies include selecting the resource with the greatest interval of time, and the resource with the fewest number of scheduled jobs. For the greatest interval abortion policy, the interval is computed from the earliest time at which the request can be started, to the latest time the request can run for the given conflict window if the conflicting jobs(s) was/were removed from the resource.

\subsection{Matchmaking Strategy for Handling Emergency Requests}

This section describes how the system handles Emergency requests. An emergency is defined as an unforeseen event demanding immediate action [MER-12]. In the scope of this research Emergency requests are modelled as Advance Reservation requests but with three main differentiating characteristics; firstly, they have the highest priority level, secondly, they are executed as soon as possible upon arriving in the system, and thirdly they have no laxity component. 


\subsubsection{Reallocation Type}

When an Emergency request pre-empts an AR request, the pre-emption policies (described in Section 3.3.3) have the pre-empted request sent back to the broker for reallocation if the following conditions are met. The first condition is that the emergency service time is known and the second condition is that the request will no longer be able to meet its deadline on the resource it is currently scheduled on after the emergency would finish. This thesis investigates two types of reallocation on another resource. The first type is pre-emptive resume. This is where the request resumes its execution from where it finished executing on the original resource. The other type is pre-emptive restart. This involves restarting the request on the new resource.

\subsubsection{Known / Unknown Emergency Service Time}

When handling Emergency requests, the behaviour of the system depends greatly on whether or not the emergency service time is known. If the emergency service time is known, the broker uses the matchmaking strategy based on knowledge of the scheduling policies described in Section 3.1 to try to schedule the incoming Emergency request without pre-empting other jobs. If this is unsuccessful, the broker uses one of the preemption policies outlined in Section 3.3.3. When the selected job is pre-empted, the scheduler uses the service time of the emergency and determines if that job is able to meet its deadline after the emergency would finish executing. If the pre-empted job is unable to meet its deadline after it would resume, the resource sends the job back to the broker for reallocation. The broker then determines if the pre-empted job is able to meet it's deadline on another resource based on the reallocation type, as discussed in Section 3.3.1. Note that the priority level of the pre-empted job remains unchanged when it is sent 
back to the broker. If the pre-empted job is still able to meet its deadline, the broker attempts to reallocate it on another resource. If the pre-empted job can no longer meet its deadline, or there is no other available resource where this job can be scheduled, the job is aborted from the system.

If the emergency service time is unknown, the broker does not use the matchmaking strategy described in Section 3.1 that requires knowledge of the emergency service time. Instead the broker uses one of the pre-emption polices described in Section 3.3.3, for selecting a resource to execute the Emergency request. When a request is pre-empted and the emergency service time is unknown, the pre-empted job is paused until the Emergency request completes. After the Emergency request is processed, the resource aborts all jobs that can no longer meet their deadlines, and begins with the first available request that can meet its deadline.

\subsubsection{Pre-emption Policies}

The pre-emption policies presented in this thesis consider the pre-emption of Advance Reservation requests to accommodate Emergency requests only. As mentioned in Section 3.2.2, Advance Reservation requests that are accepted by a resource provide a QoS guarantee that once scheduled, they will complete by their deadline. For not violating this QoS guarantee, the pre-emption policies outlined in this thesis do not consider the preemption of low priority ARs to accommodate high priority ARs. However, this QoS guarantee needs to be violated for accommodating Emergency requests. The pre-emption policies are used by the broker for selecting a resource to execute the Emergency request. The following sections describe the pre-emption policies proposed in this thesis. 


\subsubsection{Next Resource Pre-emption Pollcy (NR-P)}

As in Section 3.2.3.3, the broker keeps track of which resource from the resource list that last had a job pre-empted on it $\left(\mathrm{R}_{\mathrm{LP}}\right)$. With this policy, the broker selects the resource that appears directly after $R_{L P}$ in the resource list for executing the Emergency request.

\subsubsection{Largest Slack Pre-emption Policy (LS-P)}

As in LS-A presented in Section 3.2.3.1, slack refers to the amount of time between when the conflicting job is expected to finish and its deadline. In this case, the conflicting job refers to the job currently executing. With this policy, the broker computes the slack of the request currently executing for each of the available resources in the resource pool. The resource containing the AR request that has the largest slack is selected to execute the emergency. If there is a tie, the broker chooses the first resource from the resource list having the largest slack. Only the resources that are not currently executing emergencies are considered. If all of the resources are currently executing emergencies, this policy selects the resource with the emergency that finishes first. The motivation behind this policy is that pre-empting the request with the largest slack will allow the best chance of the request being reallocated on another resource.

\subsubsection{Greatest Service Time Pre-emption Policy (GS-P)}

In this pre-emption policy, the broker analyses the service time of the request currently executing for each of the available resources in the resource pool. The resource containing the $A R$ request with the greatest service time is selected for executing the emergency. If there is a tie, the broker chooses the first resource in the resource list that is executing the request with the greatest service time. Only resources that are not currently executing emergencies are considered. If all of the resources are currently executing 
emergencies, this policy selects the resource with the emergency that finishes first. The motivation behind this policy is that pre-empting the request with the greatest service time will minimize the number of requests that are aborted because of emergencies.

\subsubsection{Shortest Time Pre-emption Policy (ST-P)}

In this pre-emption policy, the broker computes the time the currently executing request has executed for each of the available resources in the resource pool. The resource containing the AR request that has executed for the shortest amount of time is selected for executing the emergency. If there is a tie, the broker chooses the first resource from the resource list containing the request that has executed for the shortest amount of time. Once again, only the resources that are not currently executing emergencies are considered. If all of the resources are currently executing emergencies, this policy selects the resource with the emergency that finishes first. The motivation behind this policy is that pre-empting the request that has executed for the shortest amount of time minimizes the time wasted due to pre-emption.

Other pre-emption policies using different metrics for handling Emergency requests have been simulated. However, they are not presented as they did not produce significantly different results. These other policies include selecting the resource with the greatest interval of time between when the emergency arrives and the start time of the next job queued up in the resource schedule, and the resource with the fewest number of scheduled jobs. 


\subsection{Padding}

Padding is a technique used to artificially inflate the service time of an AR request by a specific amount of time, known as the padding time (PT). This is achieved by artificially increasing the request's expected finish time (EFT), referred to as padded EFT, inherently creating idle time between the requests accepted on the resource. The padded EFT is used by the broker for determining the schedulability of incoming requests and also by the scheduler when scheduling requests on the resources. The purpose of adding padding to AR requests is that by adding additional idle time after each accepted request on the resource, Emergency requests will be handled better. The idea is that the emergencies will use up the idle time created from the padding and less requests will be aborted. As all requests except for emergencies can have padding added to them, all of the abortion and pre-emption policies outlined in this research are able to use this technique.

When padding is not used, the expected finish time (EFT) of a request is computed by the summation of the request's start time (T) and the request's service time (S). A request always finishes after the service time has completed, regardless of whether padding is used. Padding is further explained with the help of Figure 3-2 which represents the model for an AR request that uses padding. In the figure, the time between $T$ and Actual EFT represent the time it will take for the request to execute. The time between the Actual EFT and Padded EFT signifies the amount of padding time added to the request. This produces additional idle time between the next request and when the current request finishes executing if there is no emergency executing. Since a request finishes execution after its actual EFT is reached, two scenarios are possible which are handled by the next request's start time discussed in the following sub-section. 


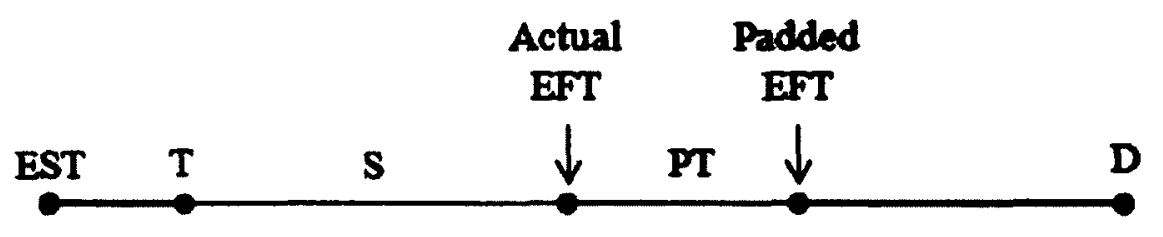

Figure 3-2: Model of AR Request with Padding

\subsubsection{Next Request Start Time}

As mentioned in Section 3.4, an AR request finishes executing when its Actual EFT is reached leaving the padding time as idle time on the resource when no emergency needs to be handled. Two cases are thus possible for determining the time at which the next scheduled request is executed. The first case involves ignoring the idle time left from the request that just finished. In this case, the next scheduled request begins immediately after the actual EFT of the completed request or at its EST, whichever comes first. Since this request has been rescheduled to run before its scheduled start time, the remaining requests in the resource schedule are rescheduled in the same way. The second scenario involves keeping the resource idle for the allotted amount of padding time, and beginning the next request when it was scheduled to start executing on the resource.

This chapter has introduced the resource management techniques used for handling both AR requests with priorities, and Emergency requests. The simulation model for analysing the performance of these techniques is presented in Chapter 4, whereas the results of the simulation experiments are discussed in Chapter 5 . 


\section{Chapter 4 - Simulation Model}

A simulator was developed for investigating the performance of the matchmaking strategies described in Chapter 3. This chapter describes the simulation model, the performance metrics, and the experiments that have been used to study the performance of the resource management techniques. The results of the simulation experiments are discussed in Chapter 5.

\subsection{System Architecture}

The system architecture that the simulator is based on consists of a group of distributed resources with a source which submits requests to various resources via a broker. The broker is responsible for determining the schedulability of the requests on the various resources. The broker decides on the resource that is best suited to perform the request based on a QoS requirement. A Grid Information Service (GIS) is the entity that the broker contacts for resource discovery services. The GIS returns a list of registered resources. It is imperative that a resource registers with a GIS once it is ready so that it can be discovered. Refer to Figure 4-1 for a representation of the system architecture used in this thesis.

The arrow entitled workflow symbolizes the transactions that take place between the broker and the resources. More specifically it involves the scheduling of AR and Emergency requests, as well as reallocating aborted requests. For a detailed explanation of how the system handles Advance Reservation requests with priorities and emergencies refer to Section 3.2 and Section 3.3 respectively. 


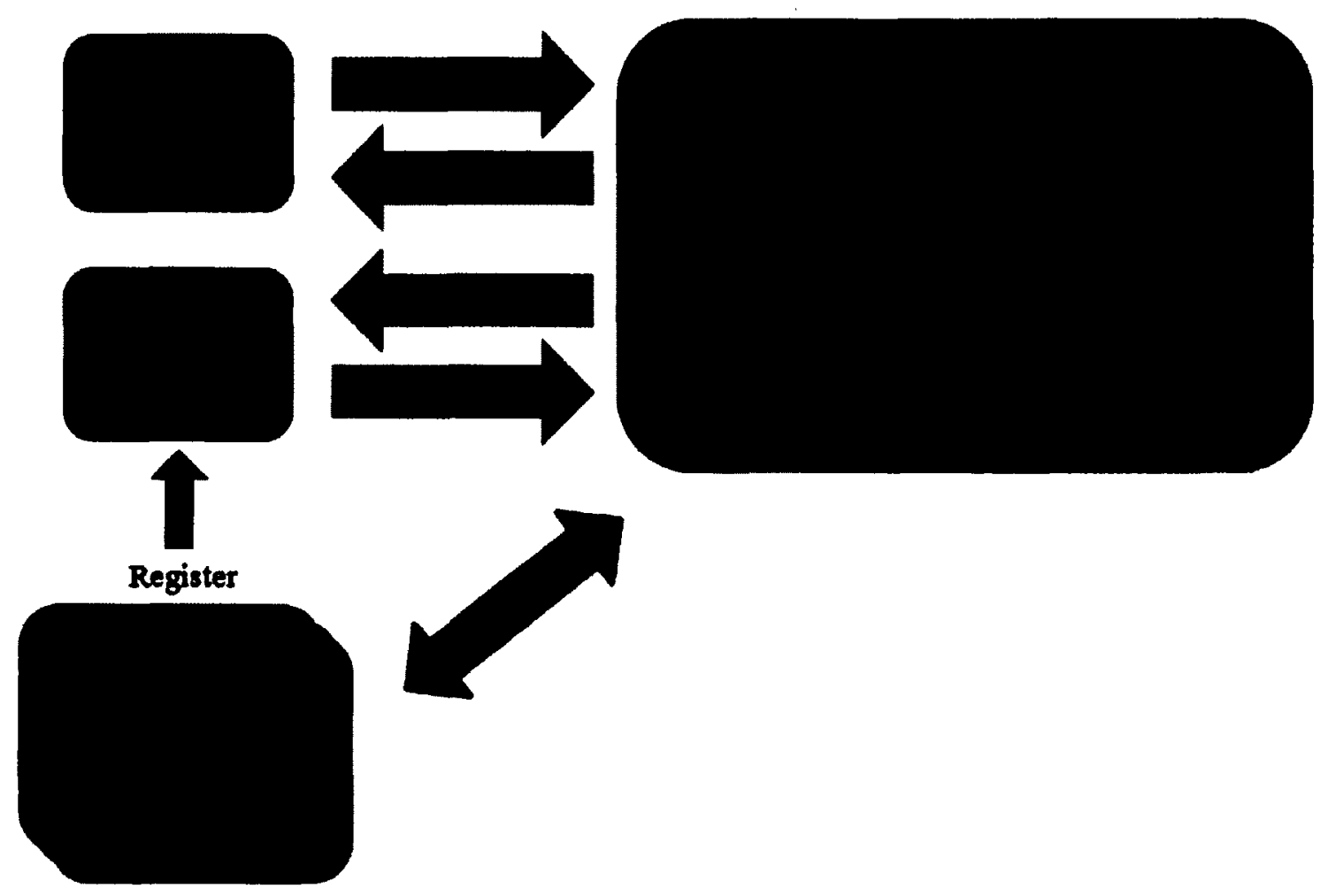

Figure 4-1: General System Architecture

\subsection{GridSim Toolkit}

Grid and cloud systems regularly include a large number of resources. The GridSim Toolkit is a tool that lets users construct simulation models of such distributed systems with an assortment of components to simulate the environment. In [BUY-02], the comprehensive features that the toolkit provides allows the simulation of different classes of heterogeneous resources, applications, users, schedulers, and resource brokers which are described in detail.

The resources and resource brokers are fully customizable to contain a certain type of operating system, a specific number of processors, and the cost of utilizing the resource. 
The toolkit also provides some standard predefined scheduling policies and also permits clients to characterize their own scheduling policies.

GridSim is built on top of existing software technologies following a modular and multilayered architecture [BUY-02]. There are five layers, as depicted in Figure 4-2. The first layer is composed of a scalable Java interface and runtime mechanism identified as the Java Virtual Machine (JVM). The second layer known as SimJava is concerned with a fundamental discrete-event based infrastructure that is constructed by utilizing the interfaces provided by the first layer. The third layer deals with simulating core GridSim entities such as information services and resources. The fourth layer focuses on the simulation of GridSim entities such as resource brokers as well as resource schedulers. The last layer is concerned with the applications that are to be used in evaluation of resource management strategies, scheduling policies, and other types of algorithms.

\subsection{Simulation Entities}

This section describes all of the entities involved in the simulation model.

\subsubsection{Source}

The source is the component that is used to generate the users requests, send them to the resource broker, and collect statistical information about the simulation. This is the entity that begins and ends the simulation. The simulation starts when the source sends the first request and ends after the last request finishes executing.

\subsubsection{Broker}

The broker is the component that receives the requests from the source. Before any resource allocation can be performed, the broker obtains a list of resources from the Grid 
Information Service (GIS). The resource management decisions regarding matchmaking and scheduling (defined in Chapter 3) are made by the broker. Using the list of resources provided by the GIS, the broker determines the resource on which the request is going to be executed. The broker also determines the start time for the request. Each resource is associated with a resource schedule containing the pending $A R$ and $E$ requests, each of which is also associated with a start time that has been established by the broker.
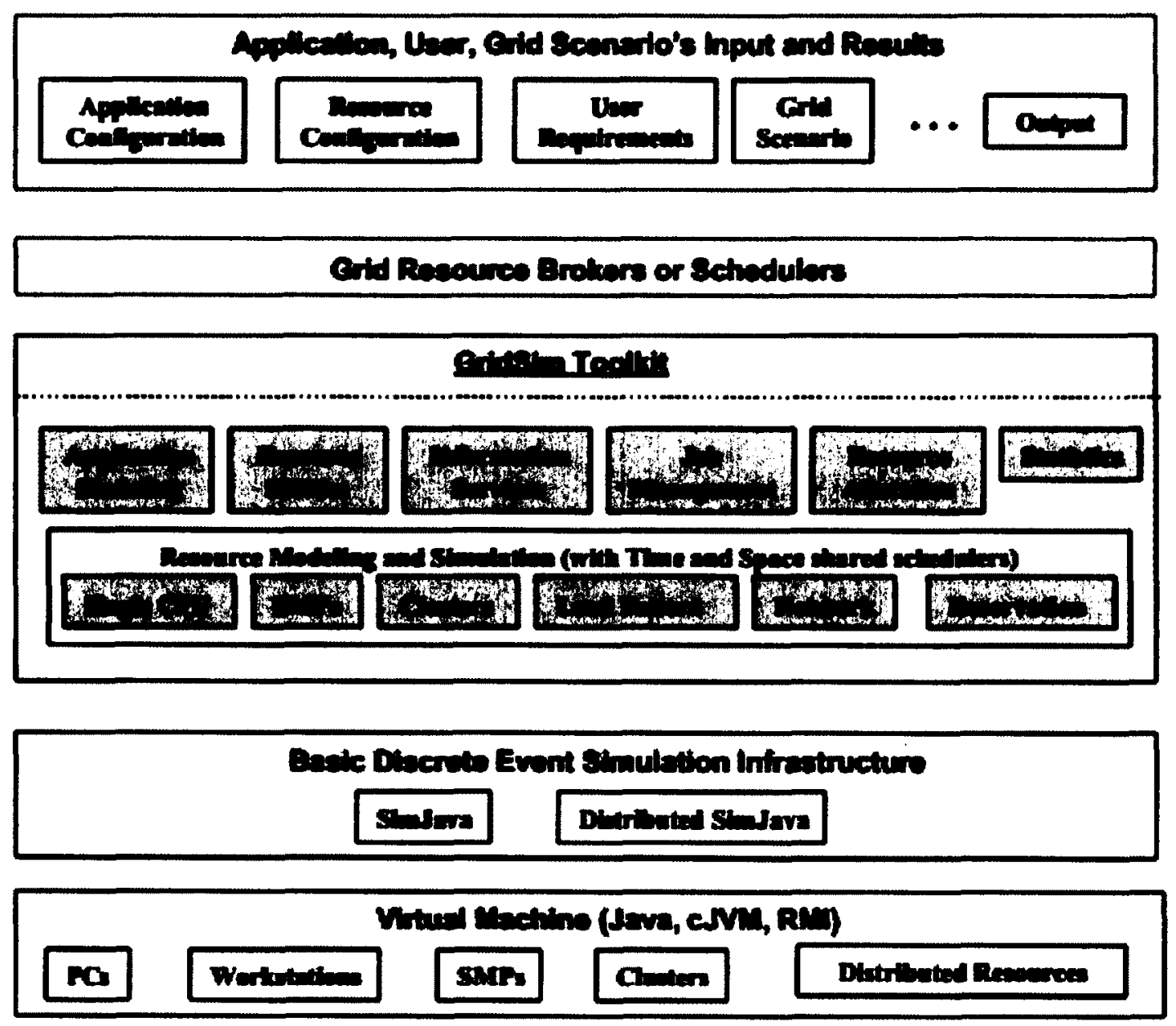

Figure 4-2: GridSim Architecture (from [BUY-02]) 


\subsubsection{Grid Information Service}

The Grid Information Service component is used for resource management in grid and cloud systems. This component is responsible for keeping track of the resources in the system. This is accomplished by the resources registering with the GIS when they become available for use within the system. The broker works together with this component to determine which resources are available for use. In order to extend the resource management across a number of entities, multiple Grid Information Service components can be distributed geographically. Since this research is concerned with the performance of the resource management techniques and not with the geographic distribution of resources, using a single GIS is appropriate.

\subsubsection{Resource}

The Resource is a component that is responsible for representing the device that performs all of the computations. This component simulates the computational requirements of the requests that are submitted from the broker. There are two types of Resources within the GridSim Toolkit. The first type of resource can handle On Demand requests only. The second type of resource can handle both On Demand and Advanced Reservation requests. The second type of resource is the one used in the simulator. This component uses several other entities to characterize the resource described in the following sub-sections.

\subsubsection{Resource Characteristics}

Every resource possesses a Resource Characteristic entity. This entity is responsible for characterising the static properties of the resource including the operating system, the number of processors available, the allocation policy, etc. The system simulated in this research extends the resource characteristic entity to also keep track of the requests that 
are scheduled at the resource. This information can be retrieved by the broker in order to perform matchmaking on the requests. In this research each resource consists of a single processor with other properties being set to default values supplied by GridSim (refer to Table 4-1).

Table 4-1: Default Values for Key Resource Characteristics Provided by GridSim

\begin{tabular}{|lcl|}
\hline IIIrilull & I Ilut & \\
\hline Architecture & Sun Ultra & Resource's hardware architecture \\
\hline Operating System & Solaris & Operating system used by the resource \\
\hline Time Zone & $9.0(\mathrm{GMT}+9)$ & Time zone the resource is in \\
\hline MIPS Rating & 377 & Resource's processing speed \\
\hline Cost & 3.0 & Cost per second to use the resource \\
\hline
\end{tabular}

\subsubsection{Resource Scheduling Policy}

The scheduling policy determines how an incoming request is scheduled on the resource. When a request arrives at the resource, the resource attempts to schedule the request based on the selected scheduling policy. Provided the schedule permits the request to meet its deadline, and does not cause other requests to miss their deadlines, the request is accepted and scheduled on the system, otherwise the request is rejected if its priority level is 0. In this research the Earliest Deadline First, described in Section 3.1.2.1, is used as the scheduling policy. If a request with a priority level greater than 0 is not accepted with the mentioned scheduling policy, the request will be scheduled based on its priority as outlined in Chapter 3. 


\subsubsection{Requests}

The GridSim Toolkit refers to requests as gridlets, but throughout this thesis they will be referred to as requests. This entity contains all of the information about the request that will be running on the resource. In the simulated system, the gridlet entity was extended to incorporate advanced features such as earliest start time, laxity, type, status, and response time. These and other request parameters regarding information about the requests are described in Section 4.5.

\subsection{Performance Metrics}

This section describes the performance metrics used to evaluate the proposed resource management techniques for a system dealing with Advanced Reservations with multiple priority levels and emergencies. Performances of these techniques are compared using the performance metrics: Probability of Blocking, Probability of Aborting, the Rate of Accepted Requests, and Average Resource Utilization for various system and workload parameters. All of the mentioned performance metrics are computed for every priority level in the system. An overall value that includes all priority levels is also computed for each performance metric. These performance metrics are discussed in the following subsections.

\subsubsection{Probability of Blocking ( $\left.\mathrm{PB}_{\mathrm{p}}\right)$}

The probability of blocking is the probability that the system will reject a request [FAR09]. The probability of blocking is also commonly referred to as the blocking ratio by other researchers [MAJ-09]. $\mathrm{PB}_{\mathrm{p}}$ is computed as the ratio between the number of requests with priority level $p$ that are rejected by the broker and the number of requests submitted on the system with priority level $p$. Since the broker is responsible for determining the 
schedulability of the system, this performance metric is calculated by the broker. Requests are rejected by the broker if the deadline of the incoming request cannot be satisfied. The probability of blocking is calculated as:

$$
\begin{gathered}
P B_{p}=(\text { Total Number of Requests } A R \text { Rejected with Priority } p / \text { Total Number of } A R \\
\text { Requests Submitted with Priority } p) * 100
\end{gathered}
$$

The overall probability of blocking, denoted as $\mathrm{PB}_{\mathrm{T}}$, is computed as:

$$
P B_{T}=\text { (Total Number of AR Requests Rejected / Total Number of AR Requests }
$$

$$
\text { Submitted) *100 }
$$

\subsubsection{Probability of Aborting (PA)}

The probability of aborting is the probability that the system will abort an accepted job before it has successfully completed [FAR-07]. PA $\mathrm{A}_{\mathrm{p}}$ is computed as the ratio between the number requests for priority level $p$ that are aborted in the system and the number of requests that were accepted with the same priority level. Accepted requests that are reallocated on another resource because they were removed from the resource that they were originally scheduled on to accommodate higher priority requests are not counted while computing in this metric. Only aborted requests that can no longer meet their deadline on any of the other available resources are considered. The probability of aborting is calculated as:

$$
\begin{gathered}
P A_{p}=(\text { Total Number of } A R \text { Requests Aborted with Priority } p / \text { Total Number of } A R \\
\text { Requests Accepted with Priority } p)^{*} 100
\end{gathered}
$$


The probability that an AR request is aborted because of an emergency is computed as:

$P A-E=($ Total number of $A R$ Requests Aborted Due to Emergencies / Total Number of AR Requests Accepted) * 100

\subsubsection{Rate of Accepted Requests $\left(A_{p}\right)$}

The Rate of Accepted Requests is the number of requests accepted by the system per unit time [MEL2-11]. $A_{p}$ is computed as the product of the arrival rate of the requests for a given priority level $p$ and the acceptance ratio associated with the same priority level. The acceptance ratio is the ratio between the number of requests with priority level $p$ that are accepted and the number of requests with priority level $p$ that are submitted into the system $\left(1-P B_{p}\right)$. The rate of accepted requests is calculated as:

$$
A_{p}=\hat{\lambda}_{p}^{*}\left(1-P B_{p}\right) \quad \text { where } \lambda_{p}=\lambda * P A R_{p}
$$

$\mathrm{PAR}_{\mathrm{p}}$ is the proportion of $A R$ requests in the system for the given priority level. Refer to Section 4.5.2.9 for a further discussion of $\mathrm{PAR}_{\mathrm{p}}$.

The overall rate of accepted requests, denoted as $A_{T}$, is computed as:

$$
A_{T}=\sum_{p} A_{p}
$$

\subsubsection{Average Resource Utilization $\left(U_{p}\right)$}

The average resource utilization caused from requests with priority level $p$ quantifies the proportion of time that the resources are busy executing these requests. The average resource utilization for requests with a given priority level is calculated as: 
$U_{p}=$ Sum of the Busy Times for Requests with Priority p on All Resources / (Simulation

Duration * Total Number of Resources)

The average resource utilization of all requests is computed as:

$$
\begin{gathered}
U_{T}=\text { Sum of Busy Units of Time for All Resources / (Simulation Duration * Total } \\
\text { Number of Resources) }
\end{gathered}
$$

\subsection{Workload and System Parameters}

This section outlines the workload and system parameters considered in this research.

\subsubsection{System Parameters}

This section presents the system parameters which are used to configure the simulation entities.

\subsubsection{Number of Resources}

This parameter refers to the number of available resources in the system for allocating requests to. This research experiments with the number of resources to investigate the impact it has on the performance of the resource management techniques. These values are similar to the values used in other works including [SUL-04], [FAR-07], and [MEL10].

\subsubsection{Allocation Algorithm}

As mentioned in Section 3.1.1, this is the algorithm used by the broker for allocating AR requests and emergencies to the resources before attempting any abortion or pre-emption techniques. The First Fit algorithm is used as the default allocation algorithm by the broker in the simulations. To study the impact of the allocation algorithm the Next Fit 
algorithm is used and the system performance achieved is compared with the performance achieved with the default algorithm. The allocation algorithms used in this research are similar to the ones used in [MEL-10], and [HOA-12].

\subsubsection{Abortion Policy}

As discussed in Section 3.2.3, this is the policy used by the broker when trying to accommodate Advanced Reservation requests with a priority level greater than 0 on a chosen resource when the request could not be scheduled using the allocation algorithm. The abortion policies investigated include Next Abortable Resource, Next Resource, Largest Sum of Slack Times, and Greatest Sum of Service Times. The details of these abortion policies can be found between Section 3.2.3.2 and Section 3.2.3.5.

\subsubsection{Pre-emption Policy}

As described in Section 3.3.3, this is the policy used by the broker to accommodate Emergency requests on for the most appropriate resource, decided by the pre-emption policy when the Emergency request cannot be scheduled using the allocation algorithm. The pre-emption policies investigated include Next Resource, Largest Slack, Greatest Service Time, and Shortest Time. The details of these pre-emption policies can be found between Section 3.3.3.1 and Section 3.3.3.4.

\subsubsection{Reallocation Type}

This parameter is only used when the system is handling emergencies and the emergency service time is known. When the pre-empted request is reallocated on a new resource, this parameter dictates whether the pre-empted job will resume where it finished executing or restart execution from the beginning. Pre-emptive resume is used as the 
default throughout the simulations. To study the impact of the reallocation type on the performance of the system, pre-emptive restart has also been tested.

\subsubsection{Known / Unknown Emergency Service Time}

This parameter is only valid when the system is handling emergencies and signifies whether or not the emergency service time is known. The associated behaviour of the system is described in Section 3.3.2. As emergencies are rare events and the time required to execute the request may be unknown at the time of arrival, the emergency service time is considered to be unknown by default. To study the impact of the this parameter on the performance of the matchmaking strategies, experiments where the emergency service time is time is known is compared to the default value.

\subsubsection{Workload Type}

This parameter refers to the type of requests in the workload that are sent to the system. Since this research focuses solely on handling Advance Reservations with multiple priorities and emergencies, on-demand requests are not considered.

\subsubsection{Workload Parameters}

The research concerned in this thesis uses an open model where a stream of requests arrive at the system. This section describes the workload parameters that are used to model the workload dispensed in the simulation experiments. In every experiment, one of these parameters are varied and the performance of the system is evaluated.

\subsubsection{Arrival Rate (ג)}

The arrival rate, referred to by the symbol $\lambda$, is the rate at which requests arrive at the system. It is described in terms of the number or arrivals/second. This research uses a 
Poisson process to model the arrival of requests. The rationale for choosing the Poisson process is given in [HAC-01]. The Poisson process has been used in many other studies related to grids and clouds for modelling request arrivals including [MIN-01], [MEL2$11]$, and [HOA-12].

\subsubsection{Service Time (S)}

This parameter refers to the amount of time required for a request to complete its task on a resource. This research uses a uniformly distributed service time for ARs with a default value that has a mean service time of 50 minutes with an upper bound and a lower bound of 90 minutes and 10 minutes respectively. A similar distribution for modelling service time was used by other researchers including [SUL-04] and [MAJ-09].

The service times of emergency requests are also modelled using a uniform distribution with a lower bound and upper bound of 5 minutes and 35 minutes, respectively.

\subsubsection{Earliest Start Time (EST)}

This parameter is defined as the earliest possible time that the AR request can begin executing on the resource. The earliest start time for ARs is computed by adding the time that the request is created with a uniformly distributed random variable in the range of 0 hours to $T_{s}$ hours, where $T_{s}=12$ hours is the default value. A similar distribution is employed in [SMI-00] and [MEL-10]. To study the impact of $T_{s}$ on the performance of the system, different values are used.

\subsubsection{Laxity (L)}

Every AR request is associated with a laxity, giving the scheduler the flexibility to order requests in a way that improves the performance of the resources. The request laxity is 
defined as the difference between the deadline and the earliest start time plus the service time. The percentage laxity of an $A R$ request is defined as the ratio between the request laxity and the mean service time of the requests. Determining request laxity is achieved by multiplying the percentage laxity with the mean service time. The percentage laxity can possess any percentage greater than zero. For example, a percentage laxity of $200 \%$ produces a laxity that is twice the size of the mean service time. Laxity is calculated as:

$$
\text { Laxity }=(\text { Percentage Laxity }) *(\text { Mean Service Time }) / 100
$$

Laxity inherently affects the deadline of the AR requests. The deadline of the AR requests can be calculated as:

$$
\text { Deadline }=\text { Earliest Start Time + Service Time + Laxity }
$$

To study the impact of laxity on the performance of the system, laxity is varied between $0 \%$ and $1000 \%$. Similar values were used by other studies including [MAJ-09], [MEL$10]$, and [HOA-12].

\subsubsection{Padding Time (PT)}

The percentage padding of an AR request is defined as the ratio of the request's padding time, described in Section 3.4, and the mean service time of the requests multiplied by 100. The percentage padding can be assigned a value between $0 \%$ and the percentage laxity associated with the request. A percentage greater than the request's percentage laxity would result in the request where its padding would surpass its deadline. Padding is calculated as:

$$
\text { Padding }=(\text { Percentage Padding }) *(\text { Mean Service Time }) / 100
$$


Padding inherently affects the AR request's expected finish time used by the broker and scheduler as mentioned in Section 3.4. The expected finish time of an AR request used by the broker and scheduler is calculated as:

$$
\text { Expected Finish Time }=\text { Start Time }+ \text { Service Time }+ \text { Padding Time }
$$

\subsubsection{Start Time For Next Request}

As mentioned in the Section 3.4.1, with padding, requests finish executing after they have completed their service time with the padding time remaining resulting in that amount of additional idle time on the resource between the time when the job completed and the start time for the next request. This parameter specifies when the next request will start. Here there are two cases. In the first case, the next request begins after the idle time has elapsed resulting in the resource being idle for the padding time given. In the second case, the next request begins immediately after the current request finishes or at its earliest start time, whichever comes first. The remaining requests in the resource schedule are then rescheduled in the same order to maintain the work conserving principle.

\subsubsection{Priority Level (p)}

This parameter denotes the number that is assigned to an AR request which sets the scheduling priority for that request provided that the request is not accepted using the allocation algorithm. The higher the importance of the request, the higher the priority level associated with that request is. In this research several performance metrics are used to better analyze the performance of the resource management techniques where each performance metric is computed for every priority level. An overall value that includes 
all priority levels is also computed for each performance metric. In order to keep the number of figures manageable, three priority levels are considered. These include:

- Priority Level 0: Advance Reservation Request with low priority $\left(\mathrm{AR}_{\mathrm{L}}\right)$

- Priority Level 1: Advance Reservation Request with high priority $\left(\mathrm{AR}_{\mathrm{H}}\right)$

- Priority Level 2: Emergency Request with highest priority (E)

\subsubsection{Proportion of Emergencies (PE)}

This parameter refers to the proportion of Emergency requests in the total number of requests that arrive on the system. System performance is studied for different values of PE by varying it between 0 , where all requests are advance reservations, and 0.1 , where $90 \%$ of the requests are advance reservations and the remaining are emergencies. The emergencies are included in the system with Advanced Reservation requests with multiple priority levels.

\subsubsection{Proportion of Advanced Reservation Requests with Priorities (PAR $)$}

This parameter refers to the proportion of $\mathrm{AR}$ requests that are associated with priority level $p$ in the total number of requests. The total number of requests is calculated as the summation of the $\mathrm{PAR}_{\mathrm{p}}$ for all priority levels in the system with the addition of PE. The overall breakdown of requests can be calculated as:

$$
\sum_{p=0}^{1} P A R_{p}=1-P E
$$

To study the impact on the proportion of AR requests with priorities, excluding emergencies, $\mathrm{PAR}_{H}$ is varied between 0 , where all $A R$ requests are low priority, and 0.3 , where $30 \%$ of the total $\mathrm{AR}$ requests have high priority and the remainder of the requests consisting of low priority. 


\subsection{Accuracy of Results}

Each simulation experiment is run long enough to allow the system to reach a steady state. Every simulation experiment is run for 10,000 request arrivals and repeated 10 times such that a confidence level of $95 \%$ with a confidence interval below $\pm 5 \%$ is achieved for any given performance metric. 


\section{Chapter 5 - Performance Evaluation of the Resource Management}

\section{Techniques}

This chapter examines the results of the different simulations carried out for evaluating the performance of the system and the resource management techniques for handling both AR requests with various priority levels described in Section 3.2 and Emergency requests presented in Section 3.3. A factor at a time approach is used to study the effect that a given parameter has on the performance. In this approach all workload and system parameters are kept at their default values except for the parameter of interest which is varied. Varying a single parameter helps identify the impact that parameter has on the performance of the system. Table 5-1 displays the default values of the system and workload parameters used in the simulations. In the table, $U(10,90)$ refers to a uniform distribution used with a lower bound of 10 minutes and an upper bound of 90 minutes.

Several of the default values in Table 5-1 including the number of resources, allocation algorithm, AR service time, earliest start time, and laxity were selected based on similar values that were used in other studies. Refer to Section 4.5 for a description of these parameters and the respective studies that used comparable values.

For many of the simulation experiments, each studying the impact that a specific workload has on system performance, only one resource is used to determine the impact of that parameter on the system. Additional resources produce similar results at arrival rates that achieve the same level of contention as one resource, thus one resource is selected as the default value. On the other hand, one resource is not enough to analyze the performance of the matchmaking strategies as their purpose is to select a resource from a 
resource pool comprising of multiple resources. For these experiments, 25 resources is used as the default value for the number of resources in the resource pool.

Through experimentation, a $\mathrm{PAR}_{\mathbf{H}}$ of $10 \%$ is observed to give a reasonable mix between the requests with various priorities. This is the reason for selecting it as the default value. As emergencies are typically rare events, a PE of $5 \%$ was selected as the default value. An emergency service time of $U(5,35)$ was selected as the default value as it provides a full range of utilization for emergency requests on 1 resource.

Table 5-1: Default Values of System and Workload Parameters

\begin{tabular}{|cc|}
\hline 'araml'ler & I allu' \\
\hline Number of Resources & 1,25 \\
\hline Resource Scheduling Policy & Earliest Deadline First (EDF) \\
\hline Allocation Algorithm & First Fit (FF) \\
\hline Abortion Policy & Next Abortable Resource (NA-A) \\
\hline Pre-emption Policy & False \\
\hline Use Padding & U(10,90) minutes \\
\hline AR Service Time & U(5,35) minutes \\
\hline Emergency Service Time & U(0,12) hours \\
\hline Earliest Start Time & $200 \%$ \\
\hline Laxity & $10 \%$ \\
\hline Proportion of High Priority AR & $5 \%$ \\
\hline Proportion of Emergencies & Advanced Reservations Only \\
\hline Workload Type & \\
\hline
\end{tabular}


As discussed in Section 3.2.2, in all of the experiments in this chapter, the source in the system generates requests and sends them to the broker. The broker uses the matchmaking strategies to determine the schedulability of the incoming requests. If the broker determines that a resource is capable of accommodating the request, the request is sent to the resource and scheduled, otherwise it is rejected from the system. When higher priority requests are accommodated, lower priority requests may be aborted and sent back to the broker for reallocation. If the broker cannot reallocate the aborted requests, they are aborted from the system. The resource executes requests based on the resource schedule. When requests finish, they are removed from the resource and the broker is notified of their completion.

This chapter is structured as follows. Section 5.1 gives the objectives of the chapter. An evaluation of the performance of the system and matchmaking strategies involving a workload of AR requests with various priorities is presented in Section 5.2. Section 5.3 offers an evaluation of the performance of the system and matchmaking strategies for handling Emergency requests. Finally, a discussion of the key observations on performance and system behaviour is presented in Section 5.4.

\subsection{Objectives}

The objectives of this chapter are to examine the performance of the matchmaking strategies presented in this thesis using a broker that has knowledge of the resource scheduling policy. Throughout the simulations, the system and workload parameters are varied in order to analyze the effects they have on various performance metrics of the system. Every simulation experiment consists of 10,000 request arrivals. The simulation scenarios presented in this thesis are repeated 10 times such that a confidence level of 
$95 \%$ with a confidence interval of $\pm 5 \%$ is achieved. All of the performance metrics reported represent the average computed over the multiple runs.

For the sake of convenience for the reader, a key set of symbols used in the performance analysis is presented in Table 5-2.

Table 5-2: List of Key Symbols

\begin{tabular}{|c|c|}
\hline Symbol & Description \\
\hline $\mathbf{A}_{\mathbf{p}}$ & Rate of Accepted Requests for Priority Level $p$ \\
\hline ES & Service Time for $\mathrm{E}$ Requests \\
\hline$\overline{\text { EST }}$ & Earliest Start Time for AR Requests \\
\hline$\overline{\mathbf{L}}$ & Request Laxity \\
\hline $\mathbf{p}$ & Priority Level \\
\hline $\mathbf{P A _ { \mathbf { L } }}$ & Probability of Aborting Low Priority AR Requests \\
\hline PA-E & Probability of Aborting AR Requests because of E Requests \\
\hline $\mathbf{P A R}_{\mathrm{p}}$ & Proportion of AR Requests for Priority Level $p$ \\
\hline $\mathbf{P B}_{\mathbf{p}}$ & Probability of Blocking Requests with Priority Level $p$ \\
\hline PE & Proportion of Emergency Requests \\
\hline PT & Padding Time \\
\hline $\mathbf{R}$ & Number of Resources in the Resource Pool \\
\hline$\overline{\mathbf{S}}$ & Service Time for AR Requests \\
\hline $\mathbf{U}_{\mathbf{p}}$ & Average Resource Utilization of Requests with Priority Level $p$ \\
\hline$\overline{V_{t, A R}}$ & Variability Factor for AR Request Service Times \\
\hline $\mathbf{V}_{\mathbf{t}, \mathbf{E}}$ & Variability Factor for E Request Service Times \\
\hline
\end{tabular}




\subsection{Performance of a System Containing ARs with Multiple Priority}

\section{Levels}

This section discusses the performance of a system consisting of Advanced Reservation (AR) requests having various priority levels. For the reasons mentioned in Section 3.2.1, only two priority levels are used for the AR requests giving rise to a low and a high priority AR request. As mentioned in Section 3.2.2, since ARs are typically executed in the future and provide a guarantee that once accepted on a resource they will complete by their deadline, ARs are not provided the ability to pre-empt other ARs regardless of priority level.

\subsubsection{Effect of the Abortion Policy}

This section presents the impact of the abortion policies on system performance. The abortion policies are used by the broker as a way of selecting a resource in a grid or cloud system where multiple resources are available to accommodate higher priority ARs. As discussed in Section 3.2, if an available resource is capable of accommodating a higher priority request as determined by the abortion policy, the request is sent to the resource. The resource aborts the conflicting job(s), schedules the incoming request, and sends the conflicting job(s) back to the broker for reallocation. If the broker deems that the incoming request cannot be accommodated on any of the available resources the broker records this and rejects the request. As described in Section 3.2.2, a request cannot be accommodated on a resource when the requests currently scheduled are conflicting with the incoming request throughout the incoming request's execution window defined in Section 3.2.2. The abortion policies used in these experiments include Largest Sum of Slack Times (LS-A), Greatest Sum of Service Times (GS-A), Next Abortable Resource 
(NA-A), and Next Resource (NR-A). The mentioned abortion policies are described in Section 3.2.3. To distinguish the abortion policies from the pre-emption policies an "-A" is appended to the acronym of the abortion policy. To determine the performance of the abortion policies under different loads, the arrival rates of the workload is varied from low to high. For all of the experiments in this section a resource pool of 25 resources is used. All other parameters are set to their default values (see Table 5-1).

As the arrival rate increases, there is a greater amount of work subjected to the system. Since the system has a limited capacity, more work causes greater contention for scheduling requests which results in more requests being rejected. Figure 5-1 and Figure 5-2 confirm that the probability of blocking values for all of the abortion policies increases as the arrival rate increases. In Figure 5-1, the probability of blocking low priority requests $\left(\mathrm{PB}_{\mathrm{L}}\right)$ is shown for all abortion policies. As these requests have the lowest priority in the system, they do not have the ability to abort other requests. Because of this, the performance of the abortion policies have comparable results at low, medium, and high arrival rates irrespective of the abortion policy used.

Figure 5-2 displays the probability of blocking high priority $A R$ requests $\left(\mathrm{PB}_{\mathrm{H}}\right)$ using the various abortion policies. In systems involving requests with multiple priority levels, the goal is to have a way of selecting a resource for accommodating requests where more higher priority requests are accepted. In the case of NR-A where only one resource is analysed to accommodate a higher priority request, the chance of accepting that request is minimized compared to the results of the other abortion policies that analyse more than one resource. Thus, NR-A results in a higher probability of blocking than the other abortion policies. This is confirmed in Figure 5-2 as NR-A exhibits the worst 
performance of all abortion policies. Unlike NR-A, the abortion policies that analyse the status of all the available resources before selecting a resource, such as LS-A and GS-A, result in the lowest $\mathrm{PB}_{\mathrm{M}}$ values. This gives rise to a greater number of higher priority requests being accepted. In the case of NA-A which analyses all resources until it finds a resource that is capable of accommodating the request, the $\mathrm{PB}_{\mathrm{H}}$ values are statistically comparable to the abortion polices that analyse the status of all resources at medium and high arrival rates. This indicates that at medium and high arrival rates, analysing the status of all resources provides no additional benefit. At low arrival rates LS-A and GS-A perform better than NA-A because although NA-A finds a resource to accommodate the incoming request, it does not use the status of all resources. Thus, one of the resources that was not analysed may give rise to a lower $\mathrm{PB}_{\mathrm{H}}$.

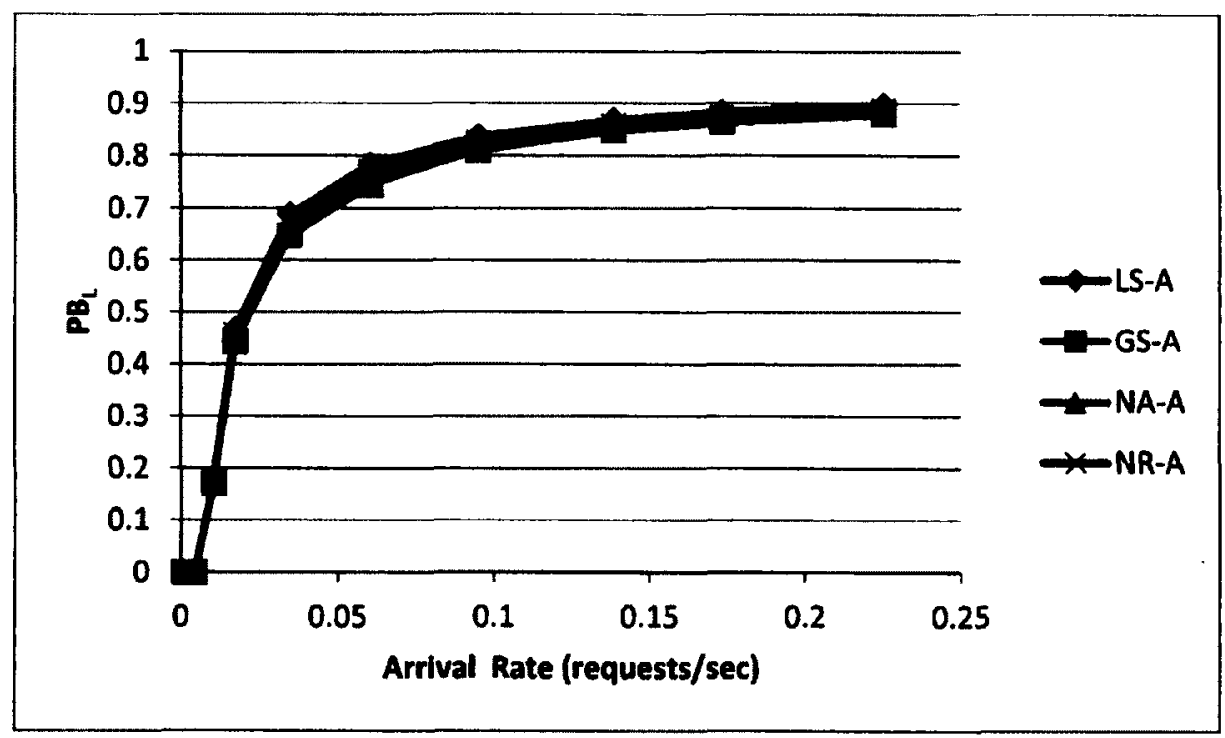

Figure 5-1: Impact of the Abortion Policies on the Probability of Blocking Low Priority ARs (PB

The total probability of blocking $\left(\mathrm{PB}_{\mathrm{T}}\right)$ represents the probability that any $\mathrm{AR}$ request is rejected regardless of priority is computed as the ratio of the total number of requests 
rejected and the total number of request submitted. Since the PB is displayed for both low and high priorities, $\mathrm{PB}_{\mathrm{T}}$ does not provide any additional information. Refer to Figure A-1 in Appendix A to view $\mathrm{PB}_{\mathrm{T}}$.

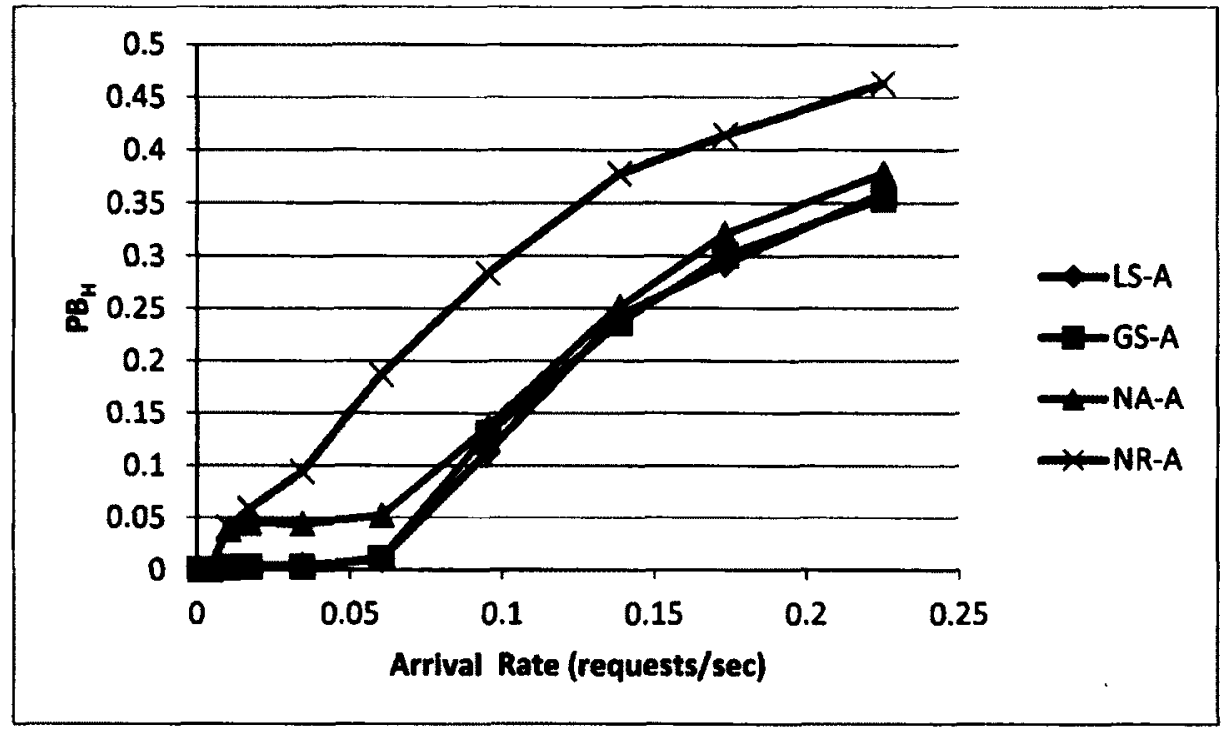

Figure 5-2: Impact of the Abortion Policies on the Probability of Blocking High Priority ARs (PB

Since the system is comprised of requests with only two priority levels, Figure 5-3 presents the probability that a low priority $\mathrm{AR}$ request is aborted $\left(\mathrm{PA}_{\mathrm{L}}\right)$ to accommodate higher priority AR requests. The results show that at low arrival rates, the values achieved with all these policies are statistically comparable to one another because contention for scheduling requests is low. As described in Section 4.4.2, the probability of aborting is computed as the ratio between the number of requests that are aborted and the number of requests that are accepted. As NR-A has the highest $\mathrm{PB}_{\mathrm{H}}$ of all abortion policies, it results in the lowest proportion of high priority AR requests that are accepted in the system. This produces the lowest probability of aborting as seen in Figure 5-3. At 
high arrival rates, $\mathrm{PA}_{\mathrm{L}}$ values achieved for all abortion policies besides NR-A are statistically comparable to one another.

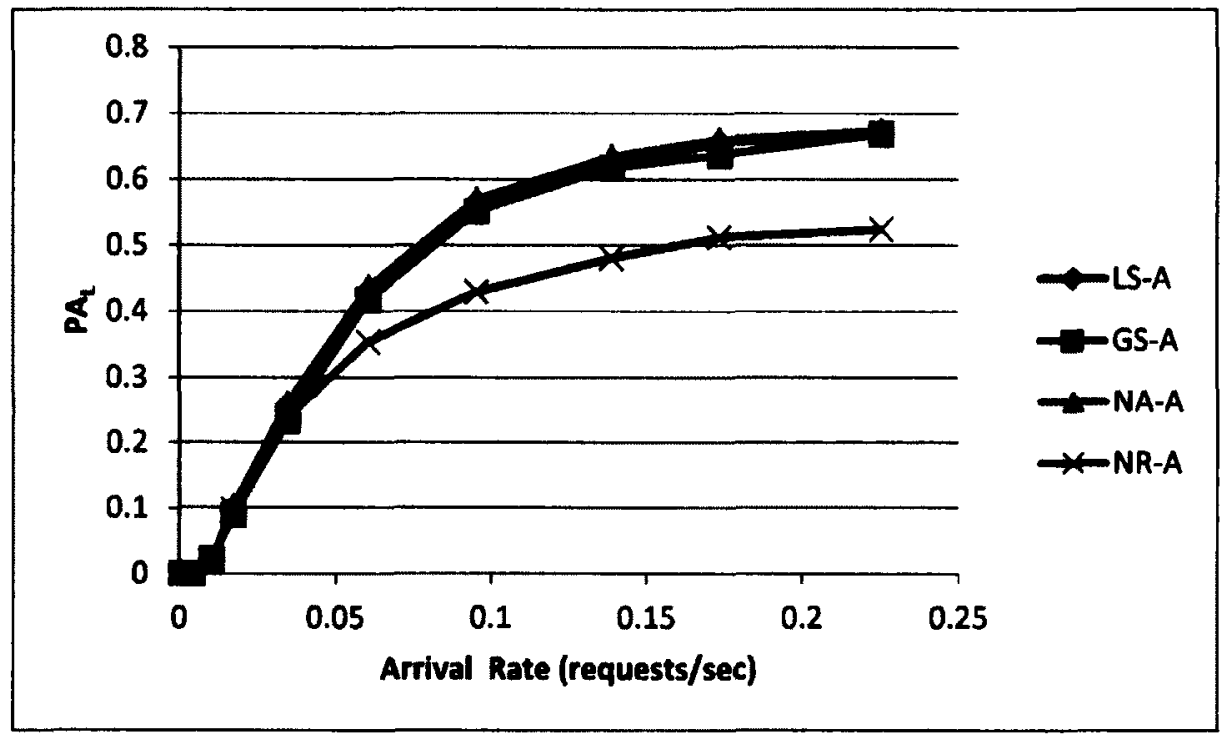

Figure 5-3: Impact of the Abortion Policies on the Probability of Aborting Low Priority ARs (PA $)$

Figure 5-4 shows the average resource utilization due to low priority AR requests $\left(\mathrm{U}_{\mathrm{L}}\right)$ for the different abortion policies. At low arrival rates, there is little contention on the resources providing a low probability of blocking. Due to this and the fact that $90 \%$ of requests in the system are low priority, the $U_{\mathrm{L}}$ values are observed to increase with the arrival rates where there is low contention. At medium and high arrival rates, the contention on the resources increase. As more higher priority requests are accepted into the system, more low priority AR requests are aborted resulting in a decrease in $U_{L}$ with an increase in arrival rate, as observed in Figure 5-4. At low arrival rates, the $U_{L}$ values for all of the abortion policies are comparable. Since NR-A has the highest $\mathrm{PB}_{\mathrm{H}}$ of all abortion policies, more low priority requests are able to execute as there are less higher priority requests aborting them. Because of this, NR-A is the abortion policy with the 
highest $U_{L}$ values at medium and high arrival rates. The remaining abortion policies have $\mathrm{U}_{\mathrm{L}}$ values that are statistically comparable to one another at medium and high arrival rates.

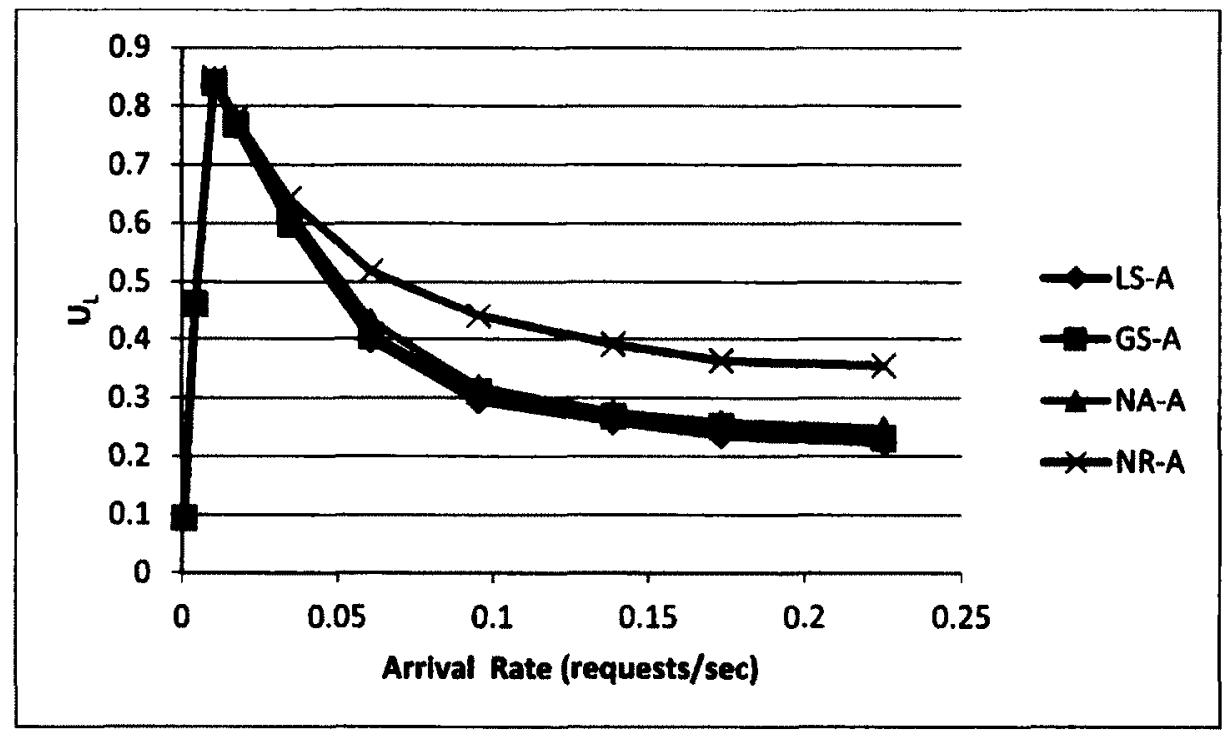

Figure 5-4: Impact of the Abortion Pollcies on the Average Resource Utilization due to Low Priority $\operatorname{ARs}\left(\mathbf{U}_{\mathbf{L}}\right)$

Figure 5-5 shows the average resource utilization due to high priority $A R$ requests $\left(U_{H}\right)$ for the different abortion policies. As the arrival rate increases, more high priority AR requests are accepted into the system resulting in a higher $U_{H}$ values. Since NR-A has the lowest $\mathrm{U}_{\mathrm{H}}$ along with the highest $\mathrm{PB}_{\mathrm{H}}$, it is the abortion policy with the worst performance. The remaining abortion policies have $U_{H}$ values that are statistically comparable to one another. However, since in NA-A the broker stops analysing the resources once it finds one that is capable of accommodating the higher priority request, it produces the lowest overhead while still giving results that are statistically comparable to the abortion policies that analyse all resources. For this reason, NA-A seems to be the most attractive abortion policy. 


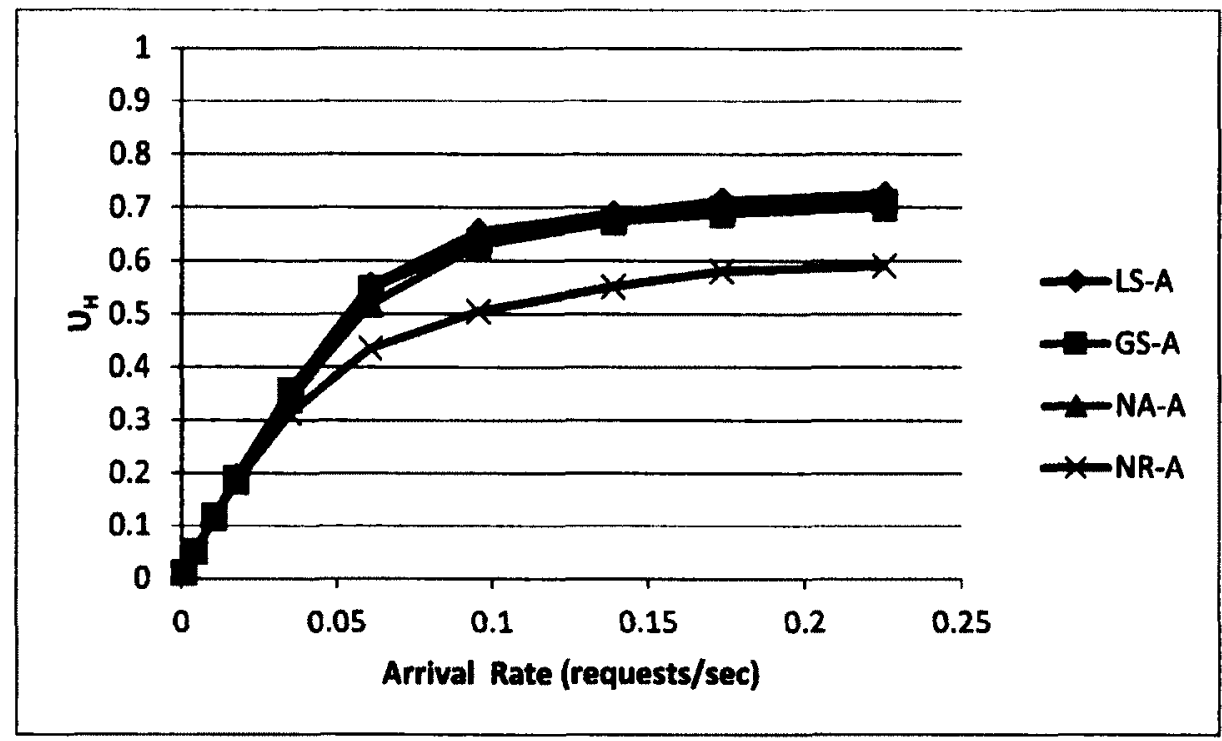

Figure 5-5: Impact of the Abortion Policies on the Average Resource Utilization due to High Priority $\operatorname{ARs}\left(\mathbf{U}_{H}\right)$

Figure 5-6 displays the average resource utilization of all requests $\left(U_{T}\right)$ regardless of priority level representing the overall utilization of the system. Here, $\mathrm{U}_{\mathrm{T}}$ is computed as the summation of $\mathrm{U}_{\mathrm{L}}$ and $\mathrm{U}_{\mathrm{H}}$ for a given arrival rate. It is observed that $\mathrm{U}_{\mathrm{T}}$ increases with the increase in arrival rate until the system saturates and becomes fully utilized which is at an arrival rate of 0.02 requests $/ \mathrm{sec}$. For arrival rates beyond this, although $U_{H}$ increases and $U_{L}$ decreases with the arrival rate, the $U_{T}$ values are approximately held at the same level as the system remains fully utilized. Since $U_{T}$ can be calculated from $U_{L}$ and $U_{H}$, it will not be shown in the other sections.

In the remaining sections involving a workload consisting of $A R$ requests with various priorities, to minimize the number of figures the overall probability of blocking $\left(\mathrm{PB}_{\mathrm{T}}\right)$ performance metric will not be displayed. As the probability of blocking for both low priority $\left(\mathrm{PB}_{\mathrm{L}}\right)$ and high priority requests $\left(\mathrm{PB}_{\mathrm{H}}\right)$ are provided, no additional information is given by including $\mathrm{PB}_{\mathrm{T}}$. Additionally, since the rate of accepted requests of both low 
priority $\left(A_{L}\right)$ and high priority $\left(A_{H}\right)$ requests are computed with $P B_{L}$ and $P B_{H}$ respectively, the graphs for $A_{L}$ and $A_{H}$ will also not be displayed in order to keep the number of figures manageable while still capturing the performance of the system. When appropriate, the graph displaying the overall rate of accepted requests $\left(A_{T}\right)$ has been included in the Appendix.

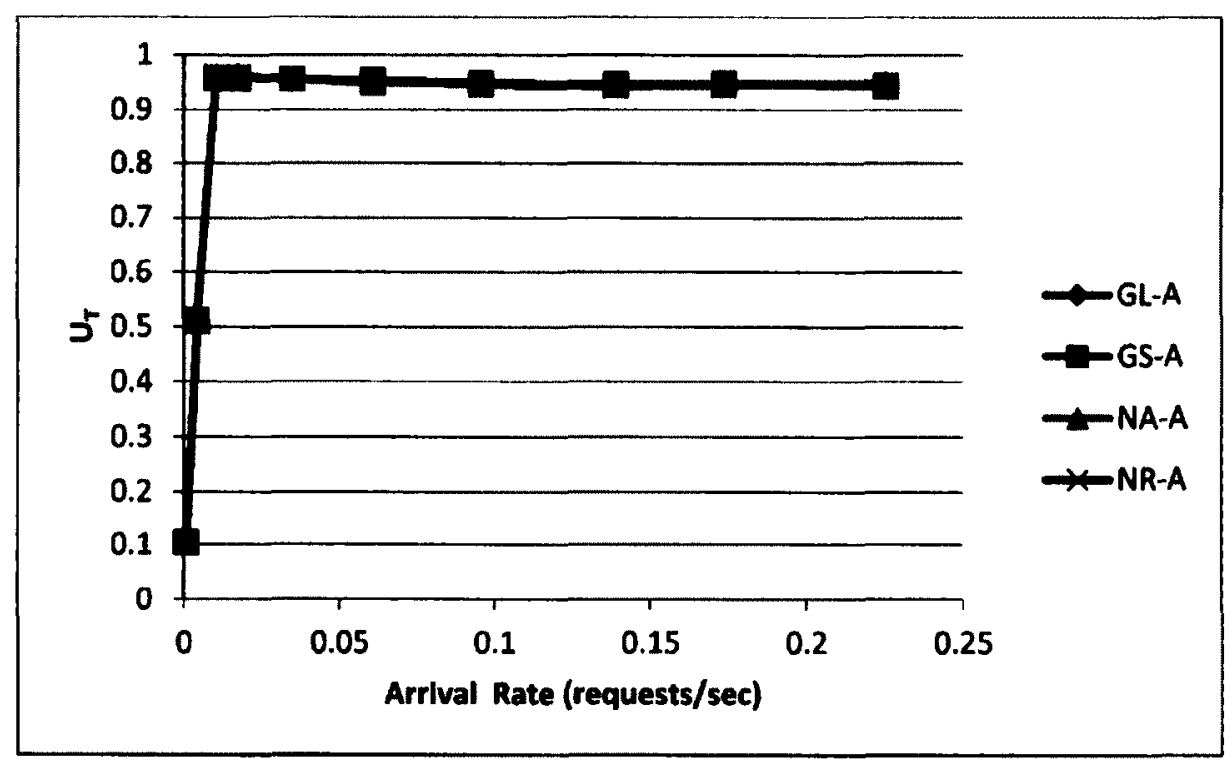

Figure 5-6: Impact of the Abortion Policies on the Overall Average Resource Utilization $\left(U_{T}\right)$

As shown throughout this section, with the exception of NR-A which produces the worst performance on the system, the performance of NA-A and the other abortion policies are statistically comparable to one another. Because of this, when analysing the performance of the workload and system parameters in the following sections, only the results for NAA which produces the lowest overhead when selecting a resource are presented.

\subsubsection{Effect of the Number of Resources}

The impact on the number of resources in the resource pool is investigated here. The number of resources, $R$, in the pool is set to 1,5 , and 10 . The other workload parameters 
are set to their default values (see Table 5-1). In this scenario the broker selects the resource based on the allocation algorithm, in this case FFAA. FFAA is outlined in Section 3.1.1.1. If the request is deemed schedulable by the broker, the request is sent to the chosen resource for execution, otherwise the broker uses the abortion policy in a further attempt to accommodate the request, in this case NA-A. A discussion of NA-A is presented in Section 3.2.3.3. If the broker finds a resource to accommodate the request, the request is sent to that resource for execution. The conflicting lower priority request(s) is/are sent back to the broker for reallocation. The purpose of having a larger resource pool is that it allows the broker more options to schedule the request. If the request cannot be accommodated on any of the available resources, the request is rejected.

Figure 5-7 shows the probability of blocking low priority requests $\left(\mathrm{PB}_{\mathrm{L}}\right)$ achieved for a various number of resources in the resource pool. As expected, the results demonstrate that for a given arrival rate, a larger resource pool rejects less requests and is thus capable of servicing more requests. As seen in figure, at very low arrival rates the requests can be handled by one resource as there is little contention. As the arrival rate increases, so does the contention on the resources resulting in more requests being rejected. However, as more resources become available more requests are accepted on the system because there are less requests being rejected. The trend for $\mathrm{PB}_{\mathrm{H}}$ is similar to $\mathrm{PB}_{\mathrm{L}}$ but with lower values for a given arrival rate as seen in Figure 5-8. It is observed that $\mathrm{PB}_{\mathrm{H}}$ values for 5 and 10 resources is close to zero for all arrival rates, signifying that 5 or more resource is enough to service the $A R_{H}$ requests. 


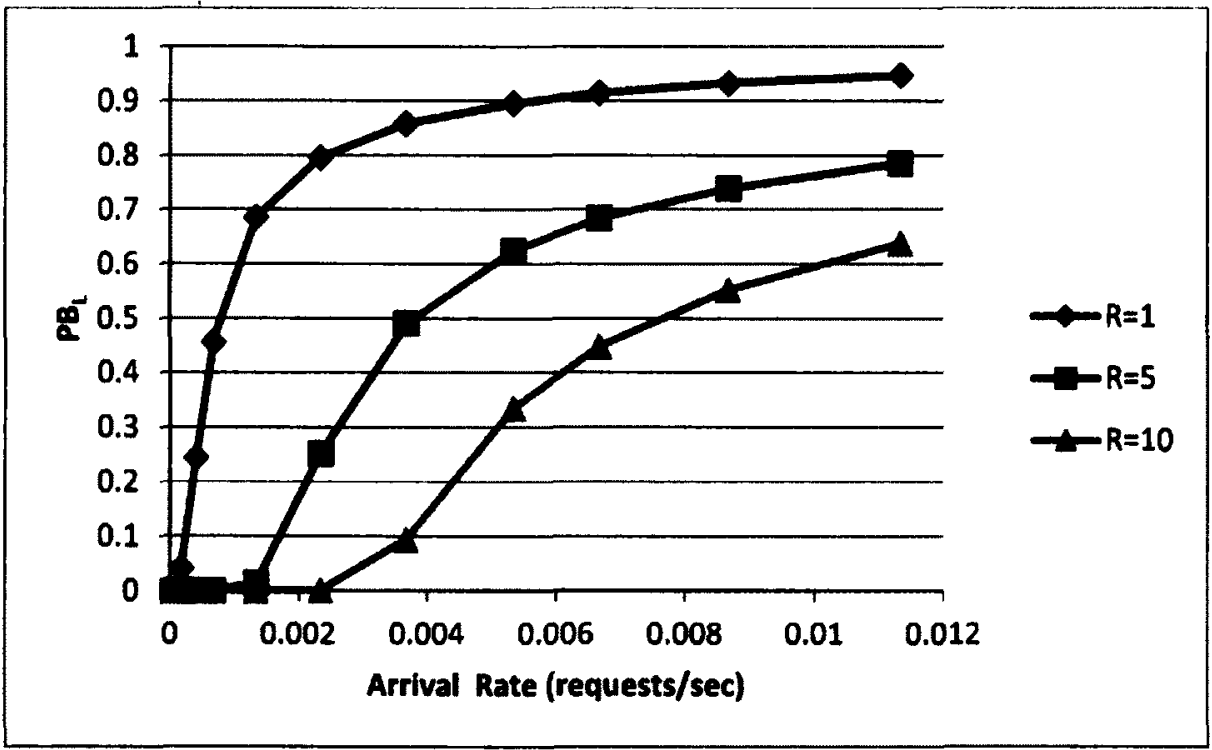

Figure 5-7: Impact of the Number of Resources (R) on the Probability of Blocking Low Priority ARs $\left(\mathbf{P B}_{\mathbf{L}}\right)$

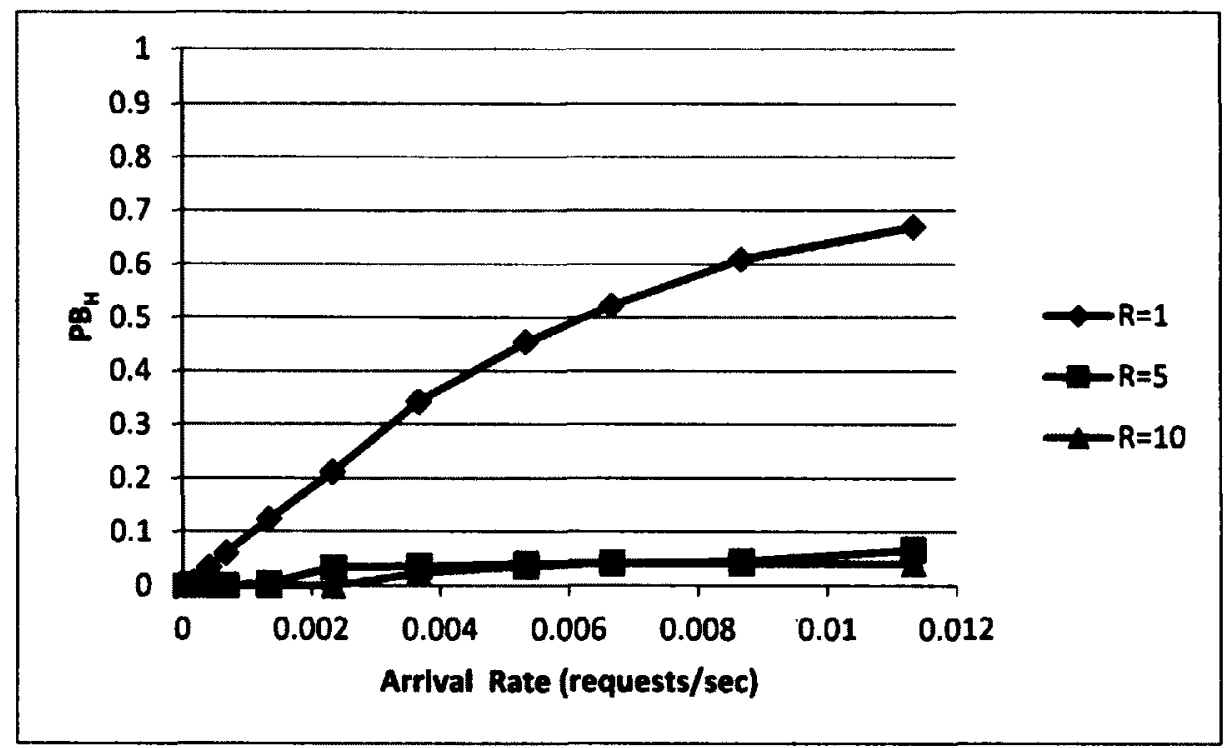

Figure 5-8: Impact of the Number of Resources (R) on the Probability of Blocking High Priority ARs $\left(\mathbf{P B}_{\mathrm{H}}\right)$

Figure 5-9 shows the effect that the size of the resource pool has on the probability of aborting requests $\left(\mathrm{PA}_{\mathrm{L}}\right)$. The figure demonstrates that for a given arrival rate, as more 
resources become available the chance of a low priority request becoming aborted decreases. One explanation for this is that as more resources become available there is an increase in flexibility when scheduling requests because more room is available. This results in lower system contention where higher priority requests have a greater chance of being accepted without having to abort lower priority requests, as shown in the figure.

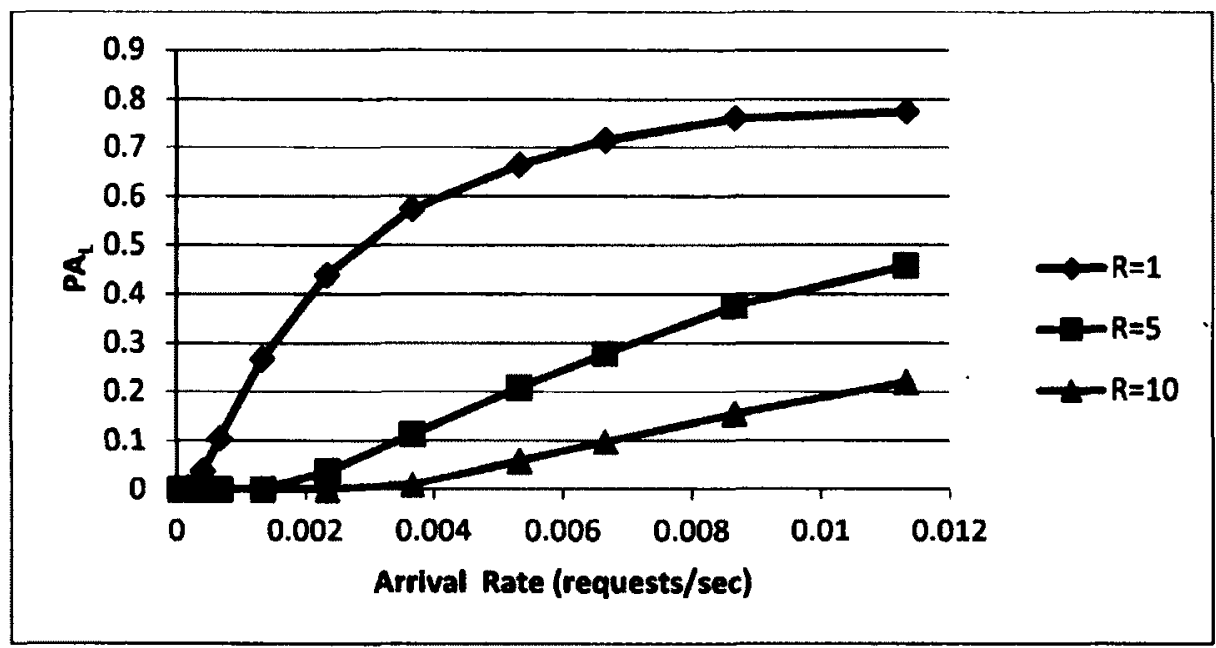

Figure 5-9: Impact of the Number of Resources (R) on the Probability of Aborting Low Priority ARs $(\mathbf{P A} \mathbf{L})$

Figure 5-10 shows the average resource utilization due to low priority AR requests $\left(U_{L}\right)$ for different number of resources. At low arrival rates, since the majority of the requests are low priority and there is little contention on the resources, the $U_{L}$ values are observed to increase as the arrival rate increases. The $U_{L}$ values increase with the arrival rate until an arrival rate reaches a point in which the system becomes saturated with requests (both low and high priority). The $U_{L}$ values decrease beyond this arrival rate because the low priority requests are aborted to accommodate higher priority requests. It is observed in the figure that as the number or resources in the resource pool increases the arrival rate required to saturate the system with requests also increases. As more resources become 
available, a greater number of requests are required to achieve the same level of contention with less resources. This is confirmed in the figure. At medium and high arrival rates, an increase in the number of resources for a given arrival rate results in an increase in $U_{L}$ values. This is due to less $A R_{L}$ requests being aborted.

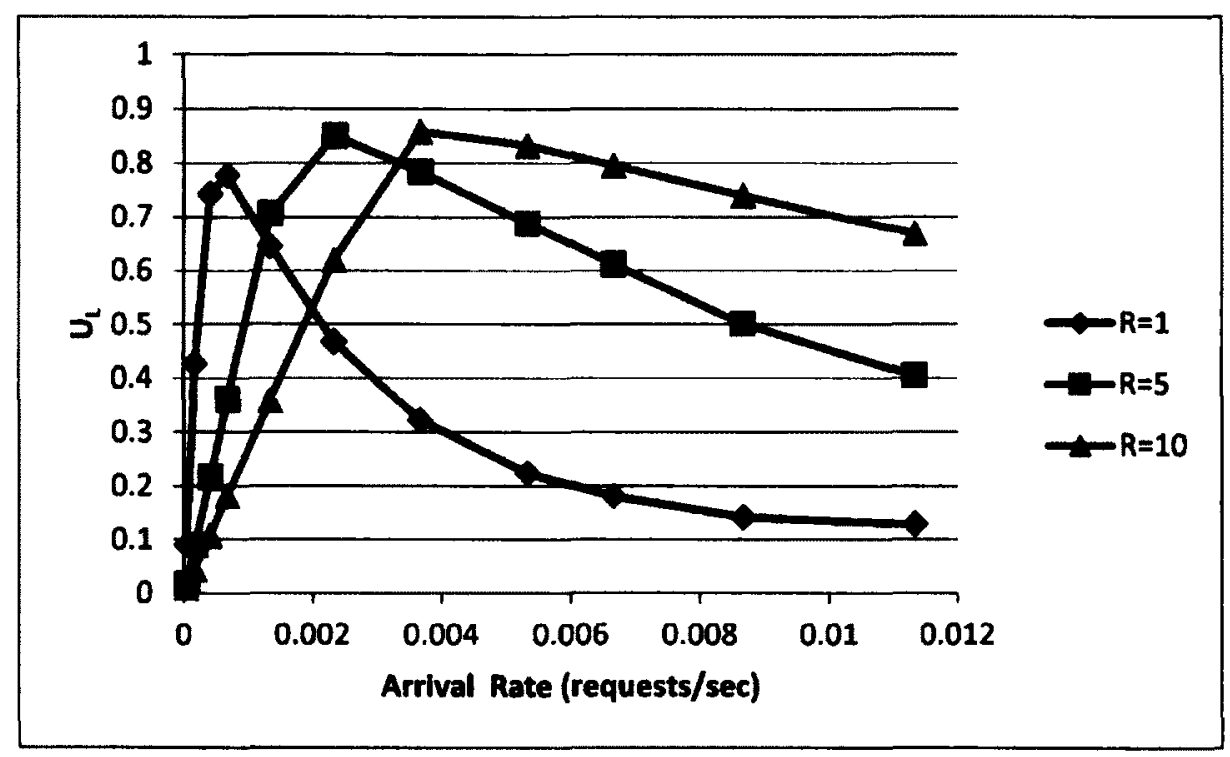

Figure 5-10: Impact of the Number of Resources (R) on the Average Resource Utilization due to Low Priority ARs $\left(\mathrm{U}_{\mathrm{L}}\right)$

Figure 5-11 shows the average resource utilization due to high priority $A R$ requests $\left(U_{H}\right)$ for a different number of resources. It is observed that for a given arrival rate, as the number of resources increase the $U_{H}$ values decrease. As observed in Figure 5-8, for 5 and 10 resources there is a low number of $\mathrm{AR}_{\mathrm{H}}$ requests that are rejected for a given arrival rate. Although more $\mathrm{AR}_{\mathrm{H}}$ requests are accepted with an increase in the number of resources, the work is divided amongst more resources and thus a lower $U_{H}$ value is achieved for a given arrival rate. 


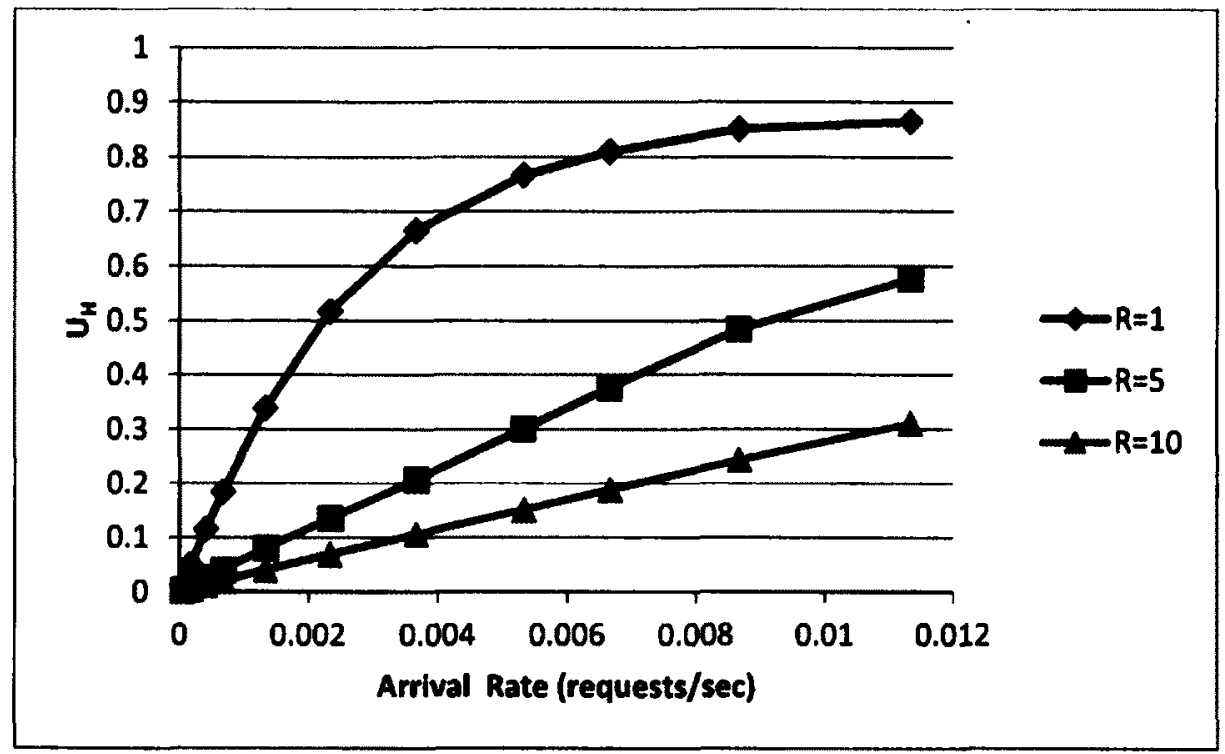

Figure 5-11: Impact of the Number of Resources (R) on the Average Resource Utilization due to High Priority ARs $\left(\mathrm{U}_{\mathrm{H}}\right)$

\subsubsection{Effect of the Allocation Algorithm}

This set of experiments studies the impact of the allocation algorithms used by the broker on system performance. The allocation algorithms used in the experiments are First Fit (FFAA) and Next Fit (NFAA), and the details of these algorithms can be found in Section 3.1.1. The number of resources in the resource pool for these experiments consist of 25 resources. All other parameters are set to their default values (see Table 5-1). The simulation results show that the allocation algorithms do not have any significant impact on $\mathrm{PA}_{\mathrm{L}}, \mathrm{PB}_{\mathrm{L}}, \mathrm{PB}_{\mathrm{H}}, \mathrm{U}_{\mathrm{L}}$, and $\mathrm{U}_{\mathrm{H}}$ observed at a given arrival rate. Thus, only the figure for PA $_{L}$ is presented (see Figure 5-12). The NFAA graph in the figure overlaps with the FFAA graph. Each of the other performance metrics listed have comparable values for a given arrival rate as that presented for the NA-A graphs in Section 5.2.1. 


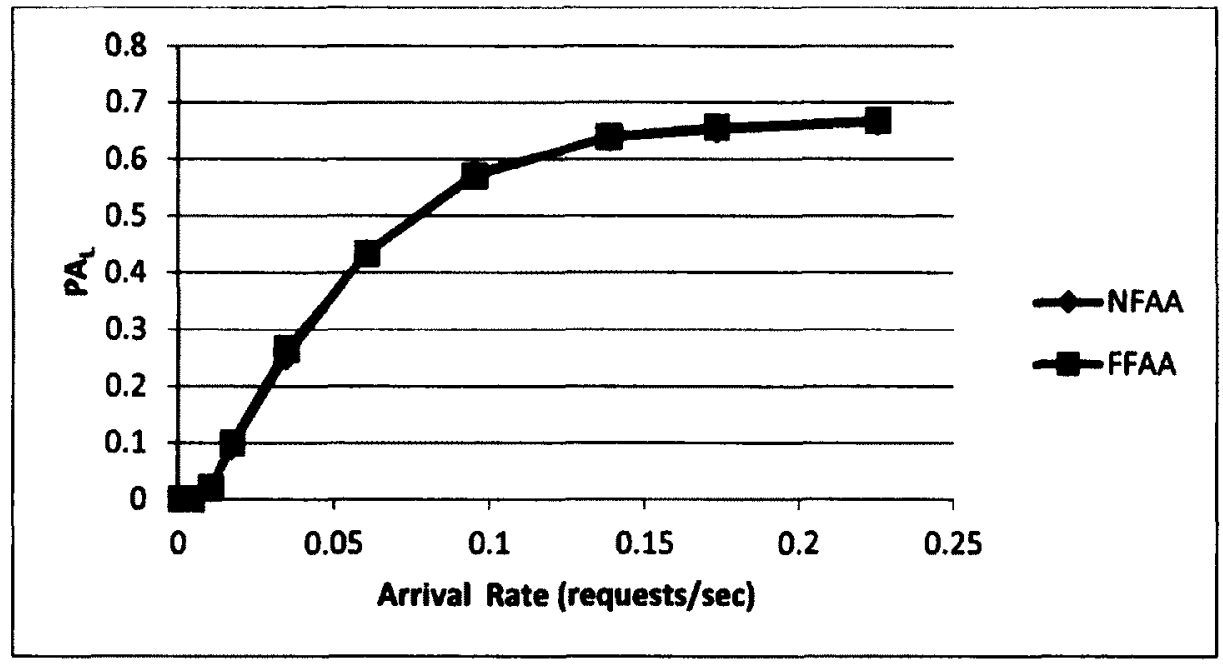

Figure 5-12: Impact of the Allocation Algorithm on the Probability of Aborting Low Priority ARs (PA )

\subsubsection{Effect of AR Service Time}

This section studies the impact of the service time for AR requests on system performance. Two experiments are conducted. One explores the effects of the mean service time on performance, while the other focuses on the impact of the variability factor. The variability factor, denoted as $V_{f, A R}$, characterizes the time between the upper and lower bound of the service times. The lower and upper bounds of an AR service time is computed as follows:

$$
\begin{aligned}
& \text { lower bound }=(1-\text { variability factor }) * \text { mean service time } \\
& \text { upper bound }=(1+\text { variability factor }) * \text { mean service time }
\end{aligned}
$$

The default value of the variability factor is set to 0.8 with the mean service time of 50 minutes using a uniform distribution. This results in request service times being generated between $(1-0.8)^{*} 50=10$ minutes and $(1+0.8)^{*} 50=90$ minutes. All other 
parameters are kept at their default values (refer to Table 5-1). The arrival rates are the same as those used in other experiments with one resource.

\subsubsection{Effect of the AR Service Time Variability Factor}

In the first set of experiments for the service time, the variability factor is varied to study the performance. $V_{f, A R}$ is used to change the upper and lower bound of the uniform distribution for generating request service times. The variability factor is changed to three different ranges resulting in three distinct levels of variability with a constant mean service time of 100 minutes. The variability factor is set to $0.02,0.5$, and 0.98 giving rise to three ranges representing low, medium, and high variability resulting in uniform distributions for the request's service time of (98 to 102 minutes), (50 to 150 minutes), and (2 to 198 minutes), respectively.

Figure 5-13 shows the impact that $\mathrm{V}_{\mathrm{f}, \mathrm{AR}}$ has on the probability of blocking high priority AR requests $\left(\mathrm{PB}_{\mathrm{H}}\right)$. The probability of blocking low priority $\mathrm{ARs}\left(\mathrm{PB}_{\mathrm{L}}\right)$ follows a similar trend and is included in Appendix A, Figure A-2.The figure shows that for a given arrival rate the $\mathrm{PB}_{\mathrm{H}}$ values are reduced for workloads with a high level of variability and increase for workloads with medium and low variability. The improvements on the probability of blocking are magnified in the rate of accepted requests. The rate of accepted requests including both low and high priorities $\left(\mathrm{A}_{\mathrm{T}}\right)$ is also included in Appendix A, Figure A-3. At low arrival rates, comparable values are obtained for the three levels of variability as there is low contention for arriving requests. The system performs best when the service time of the request has a large variability for two reasons. First, a large variability gives rise to a greater number of requests being generated with smaller service times. Second, since laxity is related to the mean service time, as 
discussed in Section 4.5.2.4, the requests with small service times have a considerably larger execution window allowing these requests more time to meet their deadlines. Workloads with low variability consist of requests having service times that are close to each other, and the mean service time. Since the percentage laxity used in the experiments is $200 \%$ of the mean service time, the laxity value is approximately double the request's service time in workloads with low variability. Compared to a workload with a high level of variability, a low level of variability limits the schedulability of the requests causing more requests to be rejected. As observed, the $\mathrm{PB}_{\mathrm{H}}$ values for a workload with a medium level of variability are slightly reduced compared to the low level of variability for a given arrival rate.

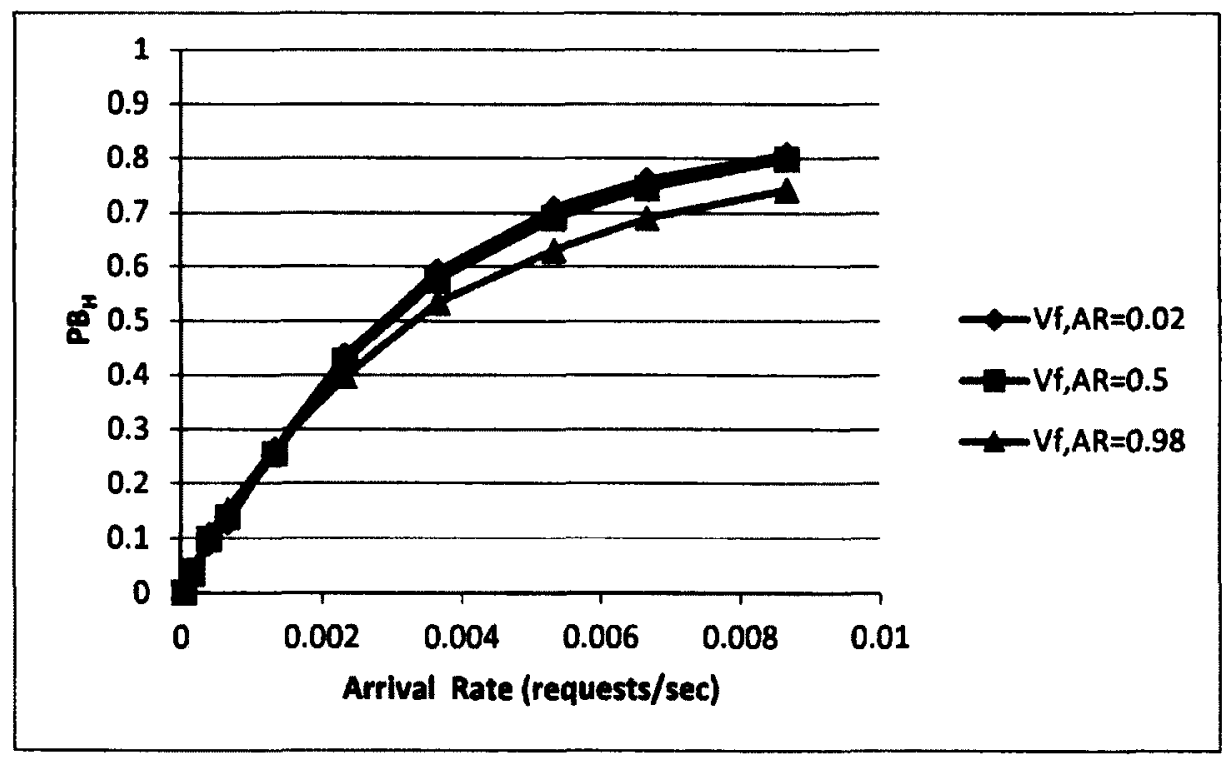

Figure 5-13: Impact of the Variability Factor for ARs $\left(V_{t, A R}\right)$ on the Probability of Blocking High Priority ARs ( $\left(\mathbf{P B}_{\mathrm{H}}\right)$

Figure 5-14 shows the impact that $V_{f, A R}$ has on the probability that low priority requests are aborted $\left(\mathrm{PA}_{\mathrm{L}}\right)$. It is observed that a high level of variability in request service time significantly diminishes the likelihood of a low priority request being aborted. As 
discussed in Section 4.4.2, the probability of aborting is computed as the ratio between the number of requests that are aborted and the number of requests that are accepted. Indicated by the logs collected during simulation runs, all three levels of variability result in a comparable number of requests for all priority levels that are aborted. But since a high level of variability produces a substantially greater number of low priority requests that are accepted into the system, it also produces a considerably lower probability of aborting requests as seen in figure.

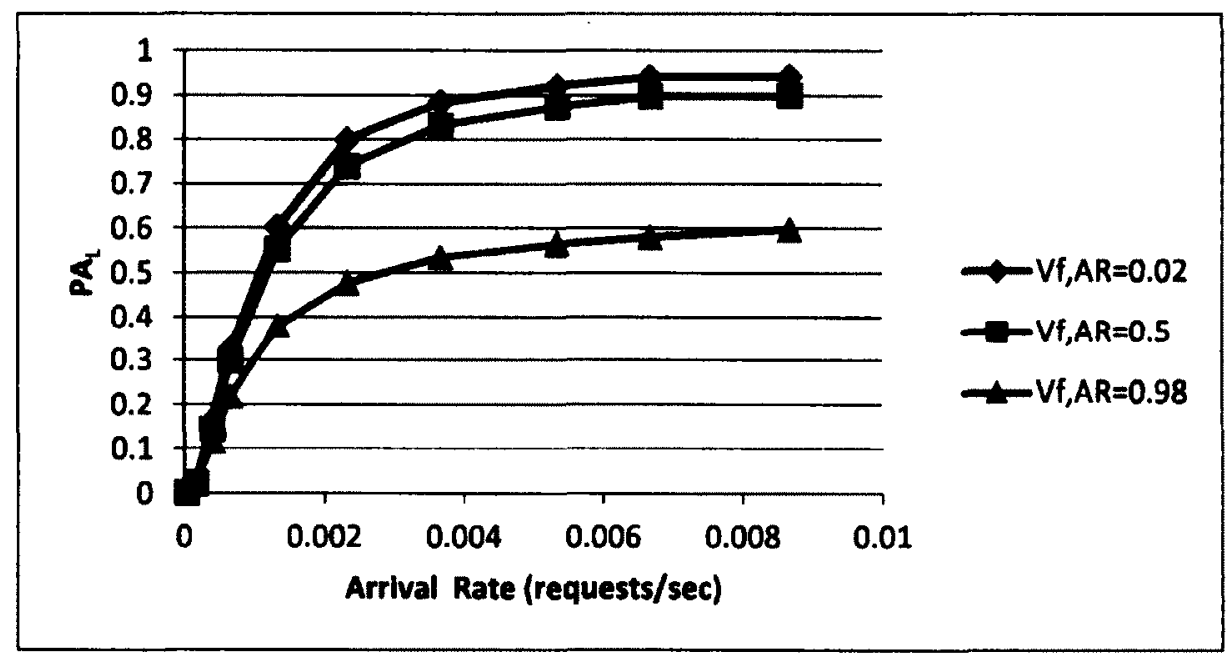

Figure 5-14: Impact of the Variability Factor for $A R s\left(V_{r, A R}\right)$ on the Probability of Aborting Low Priority ARs (PA $\left.\mathbf{A}_{\mathbf{L}}\right)$

Figure 5-15 shows the average resource utilization of high priority ARs $\left(U_{H}\right)$. The average resource utilization from low priority ARs $\left(U_{L}\right)$ which displays a trend similar to the one captured in Figure 5-4 and is included in Appendix A, Figure A-4. As discussed above, since there is a greater number of requests being generated with smaller service times as $V_{f, A R}$ increases, the requests spend less time executing on the resources due to the smaller service times resulting in slightly lower $U_{H}$ values. 


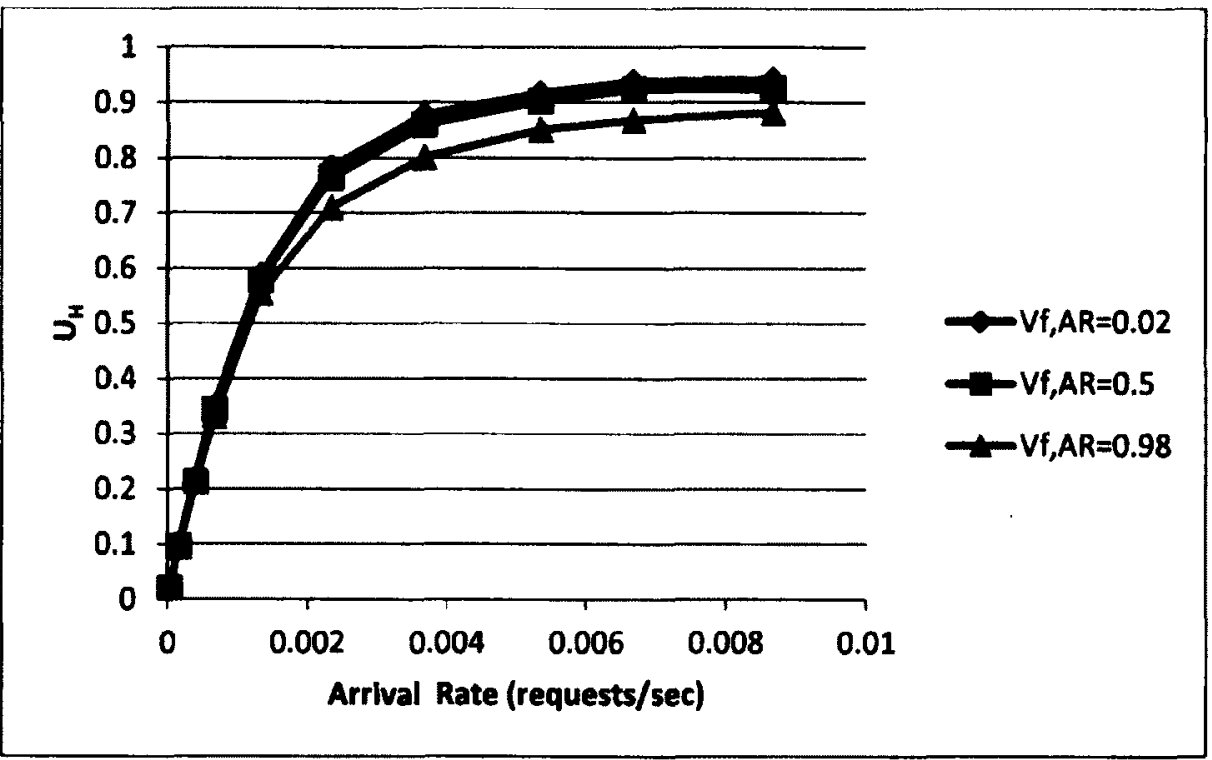

Figure 5-15: Impact of the Variability Factor for $A R s\left(V_{\mathrm{r}, A R}\right)$ on the Average Resource Utilization due to High Priority ARs $\left(\mathrm{U}_{\mathrm{H}}\right)$

\subsubsection{Effect of Mean AR Service Time}

This set of experiments focuses on studying the impact of mean service time for AR requests by varying the mean service time and keeping the variability factor constant at the default value of 0.8 . Three levels of mean service times are studied. The low level mean service time is set to 10 minutes thereby uniformly generating requests with service times between 2 and 18 minutes. The medium level mean service time is set to 50 minutes thereby generating requests with a service time between 10 and 90 minutes. The high-level mean service time is set to 200 minutes thereby generating requests with service times between 40 and 360 minutes.

Figure 5-16 shows the probability of blocking high priority requests $\left(\mathrm{PB}_{\mathrm{H}}\right)$ for various mean AR service times. The probability of blocking low priority requests $\left(\mathrm{PB}_{\mathrm{L}}\right)$ follows a similar trend as $\mathrm{PB}_{\mathrm{H}}$ (see Appendix A, Figure A-5) and can be explained in the same way as $\mathrm{PB}_{\mathrm{H}}$. Figure 5-16 indicates that for a given arrival rate, an increasing mean $\mathrm{AR}$ service 
time gives rise to higher $\mathrm{PB}_{\mathrm{H}}$ values. This outcome is intuitive since a workload consisting of requests with smaller service times utilize less time on the resource and have less of a chance of impeding other requests. The improvements in $\mathrm{PB}_{\mathrm{H}}$ are magnified in the rate of accepted requests. The overall rate of accepted requests $\left(A_{T}\right)$ which includes both low and high priority requests is included in Appendix A, Figure A6. In the experiments, the highest values of $A_{T}$ are acquired for the highest arrival rate with the lowest mean service time.

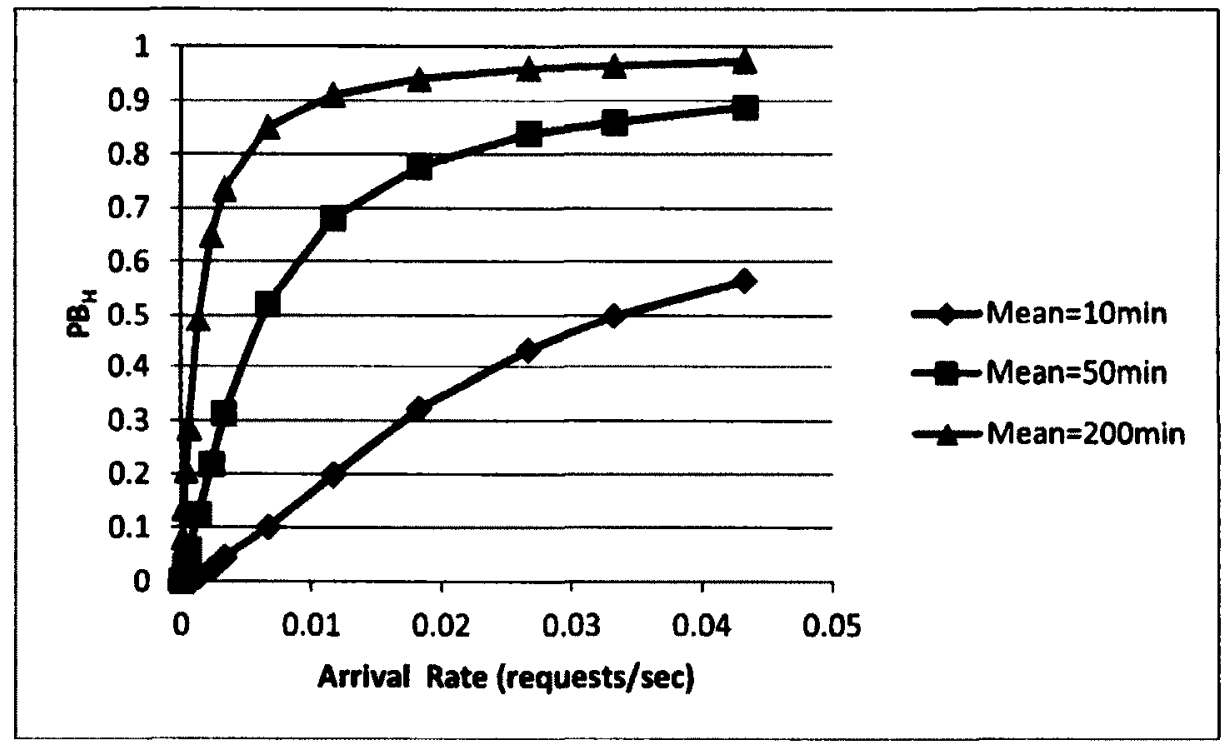

Figure 5-16: Impact of the Mean AR Service Time on the Probability of Blocking High Priority ARs $\left(\mathbf{P B}_{\mathbf{H}}\right)$

Figure 5-17 presents the probability of aborting requests $\left(\mathrm{PA}_{\mathrm{L}}\right)$ for this experiment. As expected, for a given arrival rate increasing the mean AR service time results in an increase in the number of low priority requests that are aborted. This is observed in figure. In addition, as the mean service time increases for a given arrival rate, it will take longer to service the requests. Since $A R_{H}$ requests are given priority over $A R_{L}$ requests, the average resource utilization due to high priority requests $\left(U_{H}\right)$ increases as the mean 
service time increases. This is confirmed in Figure 5-18. The average resource utilization of low priority requests $\left(U_{L}\right)$ exhibits a trend similar to the one captured in Figure 5-4 and is included in Appendix A, Figure A-7.

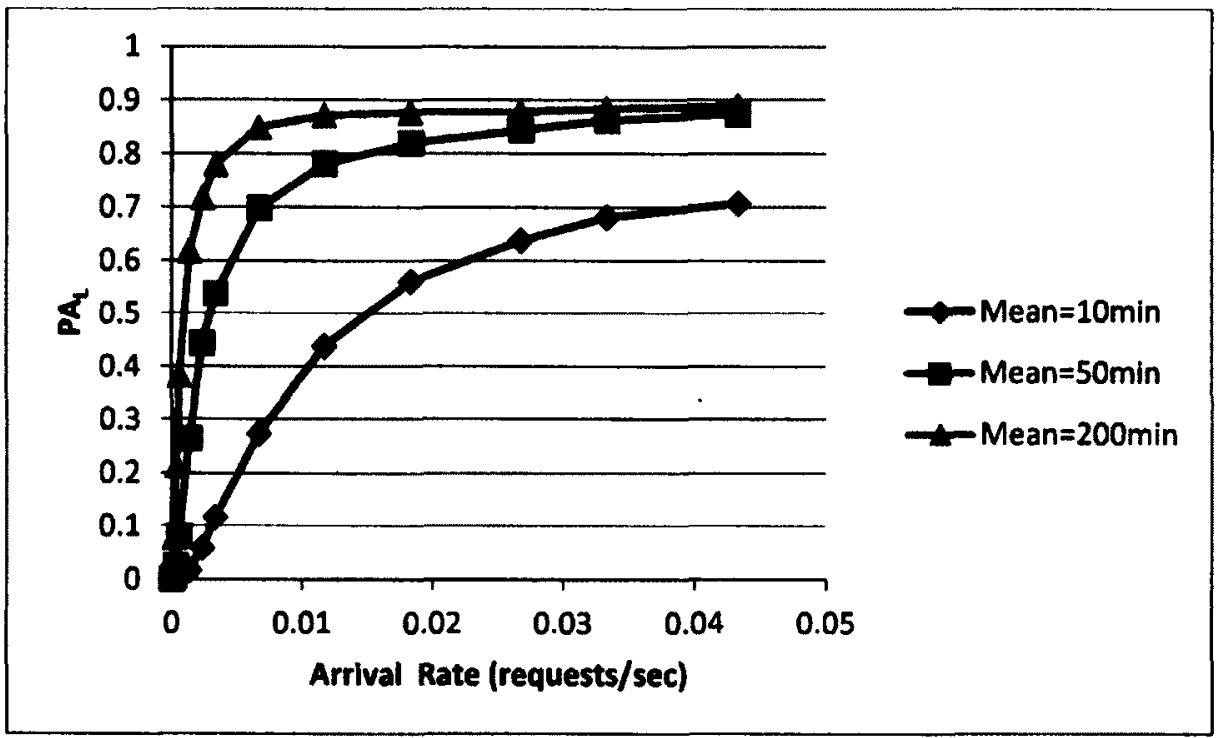

Figure 5-17: Impact of the Mean AR Service Time on the Probability of Aborting Low Priority ARs $\left(\mathbf{P A} \mathbf{A}_{\mathbf{L}}\right)$

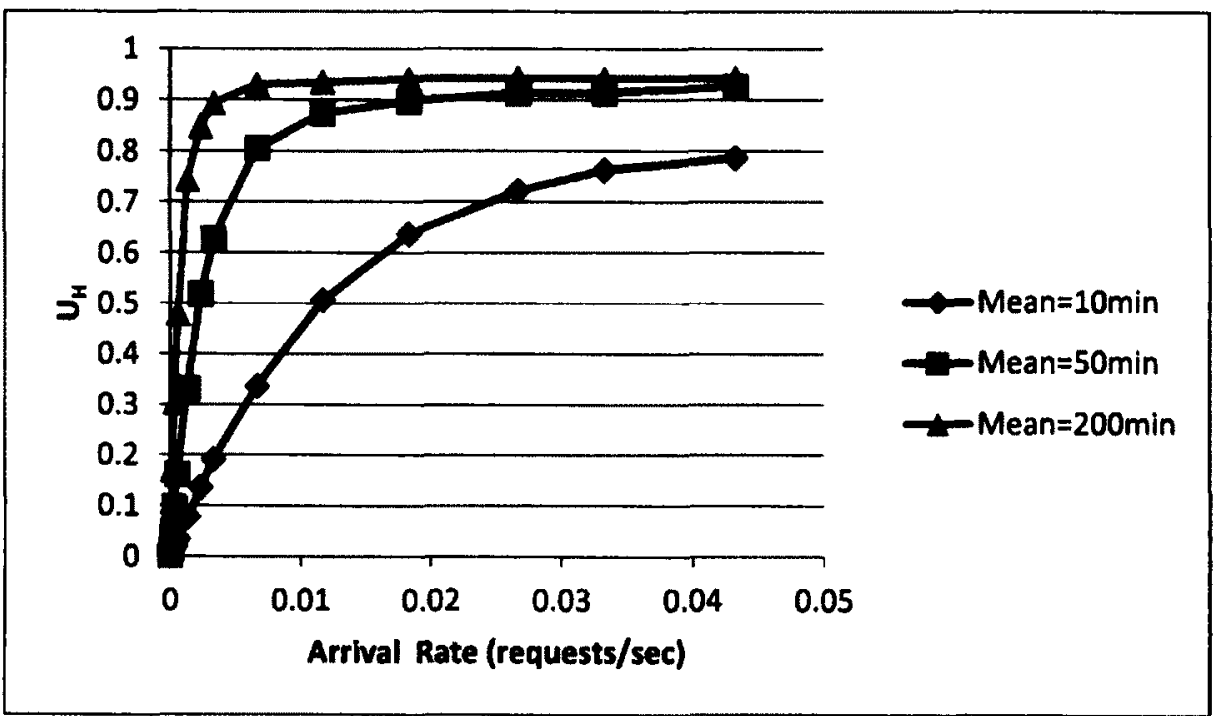

Figure 5-18: Impact of the Mean AR Service Time on the Average Resource Utilization due to High Priority ARs $\left(\mathbf{U}_{\mathrm{H}}\right)$ 


\subsubsection{Effect of Earliest Start Time}

This section presents the impact of the request's earliest start time (EST) on system performance. The earliest start time for a request is generated by taking the sum of the requests arrival time and a value generated from the uniform distribution with a lower bound of 0 hours and an upper bound of $T_{s}$ hours, where $T_{s}=12$ hours by default. The experiments performed in this section involve varying $T_{\mathrm{s}}$. These values are set to 1,5 , and 20 hours. All other parameters are kept at the default values (refer to Table 5-1). The arrival rates are the same as those used in other experiments with one resource.

Figure 5-19 shows the probability of blocking high priority requests $\left(\mathrm{PB}_{\mathrm{H}}\right)$ for different $T_{s}$ values. The probability of blocking low priority requests $\left(\mathrm{PB}_{\mathrm{L}}\right)$ follows a similar trend as $\mathrm{PB}_{\mathrm{H}}$ (see Appendix A, Figure A-8). The figure shows that as $\mathrm{T}_{\mathrm{s}}$ increases, the probability of rejecting an AR request decreases on the system. At low arrival rates, for every $T_{s}$ value, the $\mathrm{PB}_{\mathrm{H}}$ values are statistically comparable as there is little to no contention for scheduling requests. At medium and high arrival rates, $\mathrm{PB}_{\mathrm{H}}$ decreases with $T_{s}$. One reason for this is that at low $T_{s}$ values, requests commence execution sooner and therefore increase contention at the resources. As $T_{s}$ increases, the start times of the requests are spread out allowing more flexibility for the scheduler to order the requests. The improvements on the probability of blocking are magnified in the rate of accepted requests. The overall rate of accepted requests $\left(\mathrm{A}_{\mathrm{T}}\right)$ which includes both low and high priority requests is included in Appendix A, Figure A-9.

Figure 5-20 shows the probability that low priority requests are aborted $\left(\mathrm{PA}_{\mathrm{L}}\right)$ for the various $T_{s}$ values. For a given arrival rate, the $P A_{L}$ values where $T_{s}$ is 5 hours and above are statistically comparable. The figure shows that for a given arrival rate, a lower $T_{s}$ 
value gives rise to a lower probability that a low priority request is aborted. Requests with a lower earliest start time remain on the resource schedule for a shorter amount of time. The shorter the amount of time the request is on the resource schedule, the less likely the request is aborted. This is confirmed in Figure 5-21 which shows the average resource utilization values for high priority requests $\left(U_{H}\right)$ for this experiment. With a lower $P A_{L}$, more low priority requests will be served by the system producing lower $U_{H}$ as seen in the figure. The improvements in $U_{H}$ as $T_{s}$ increases is most notable at medium arrival rates. At very low and very high arrival rates, the values of $U_{H}$ are statistically comparable irregardless of $T_{s}$. $T_{s}$ does not affect $U_{H}$ at high arrival rates because the system becomes saturated with high priority requests, or at low arrival rates because requests do not arrive fast enough. The average resource utilization due to low priority requests $\left(U_{L}\right)$ displays a trend similar to the one captured in Figure 5-4 and is included in Appendix A, Figure A-10.

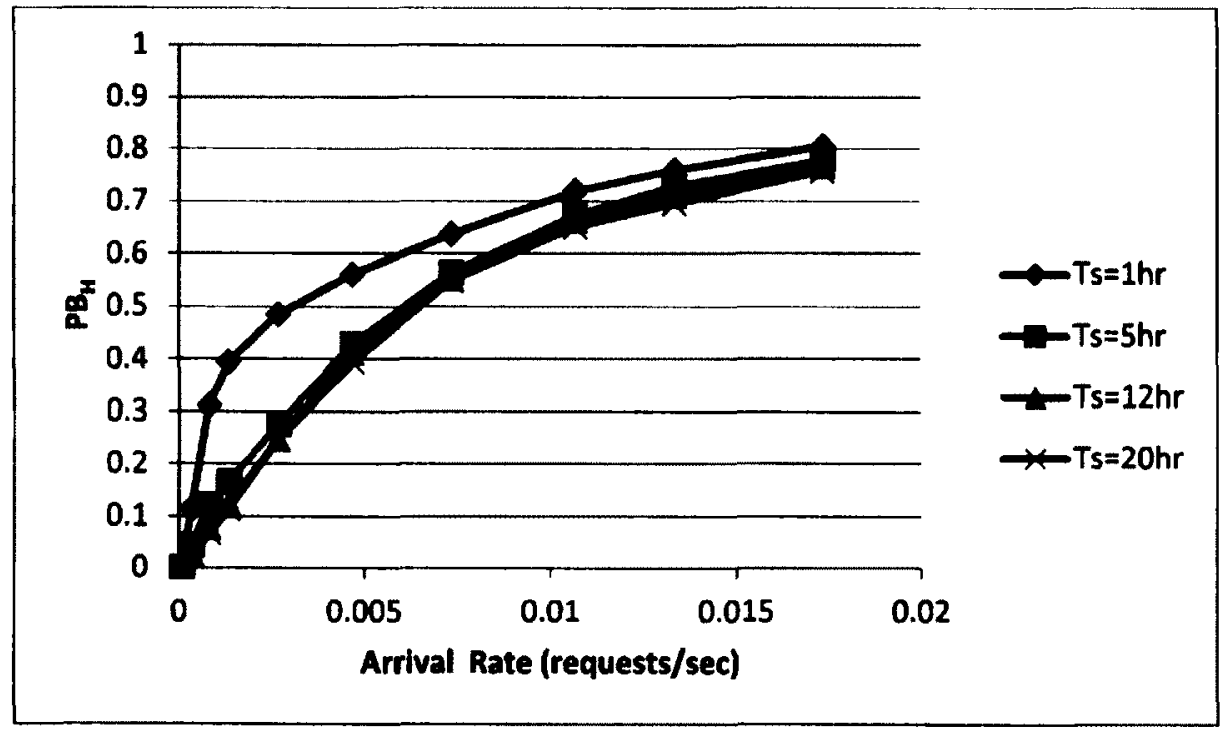

Figure 5-19: Impact of $T$, on the Probability of Blocking High Priority ARs $\left(\mathbf{P B}_{\mathbf{H}}\right)$ 


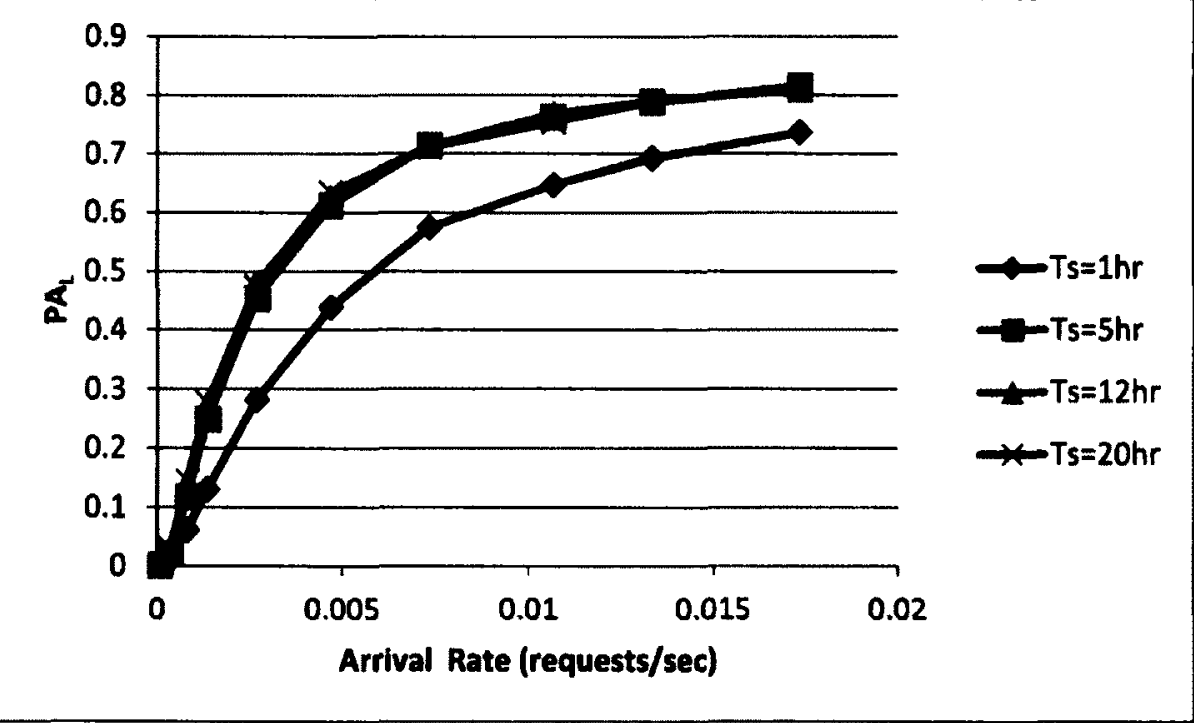

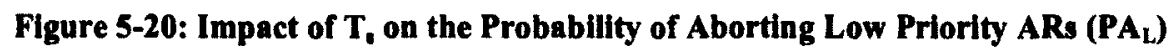

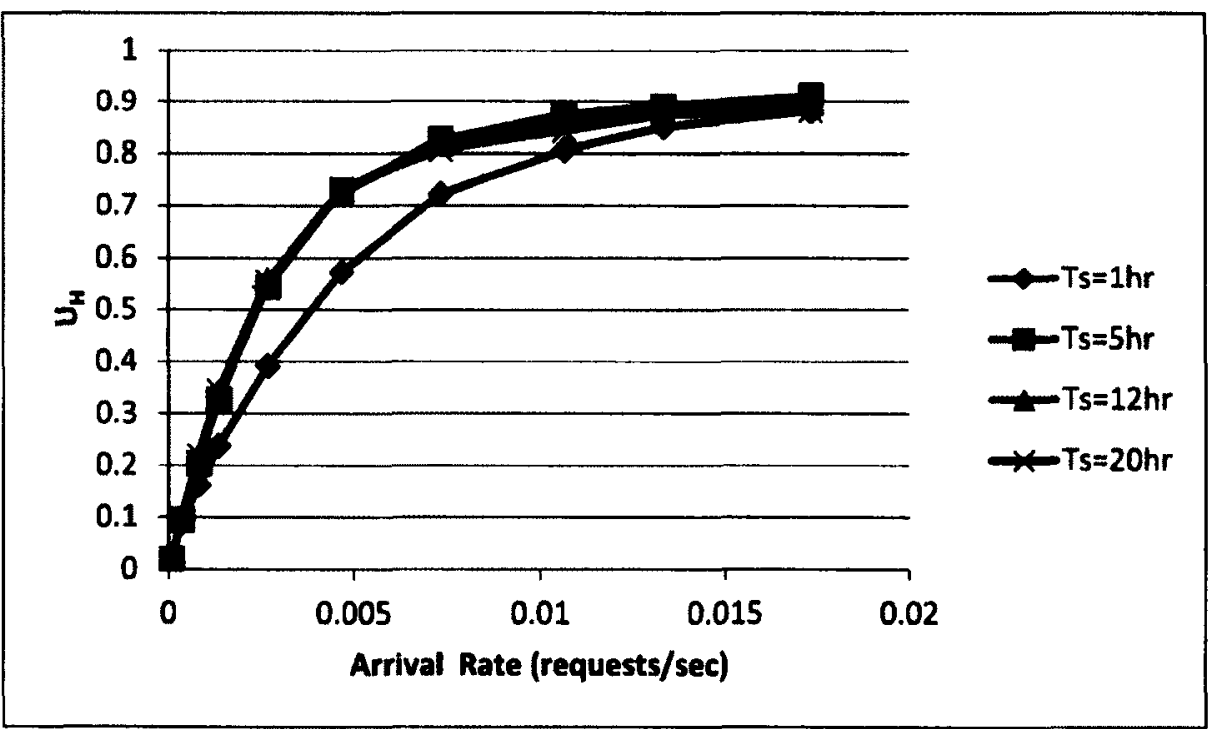

Figure 5-21: Impact of $T_{3}$ on the Average Resource Utilization due to High Priority ARs $\left(U_{H}\right)$

\subsubsection{Effect of Laxity}

This section analyzes the impact of the request's laxity on system performance. In this set of experiments, the mean percentage laxity (L) defined in Section 4.5.2.4 is varied to subject the system to different workloads. The values are set to $50 \%, 200 \%, 500 \%$ and 
$1000 \%$. All other parameters are set to the default values (refer to Table 5-1). The arrival rate is varied in the same way as the other experiments with one resource. Figure 5-22 shows the probability of blocking high priority requests $\left(\mathrm{PB}_{\mathrm{H}}\right)$ for various laxities. Altering the laxity values of the request inherently adjusts its deadline providing the broker with more or less flexibility to schedule the request depending on whether the laxity increased or decreased. With an increase in laxity, the resource where the request is scheduled is given more time to complete the request; with a decrease in laxity the reverse is true. As seen in Figure 5-22, for medium and high arrival rates, an increase in laxity results in a decrease in $\mathrm{PB}_{\mathrm{H}}$ values. As a result, there is a greater chance of the requests being accepted into the system. The decrease in $\mathrm{PB}_{\mathrm{H}}$ is most prominent in the medium ranges for the arrival rate. At very low and very high arrival rates the difference in $\mathrm{PB}_{\mathrm{H}}$ achieved for different laxity values reduces. At low arrival rates the requests do not arrive quick enough to cause substantial overlapping of requests, and at high arrival rates the $A R_{H}$ requests tend to saturate the system.

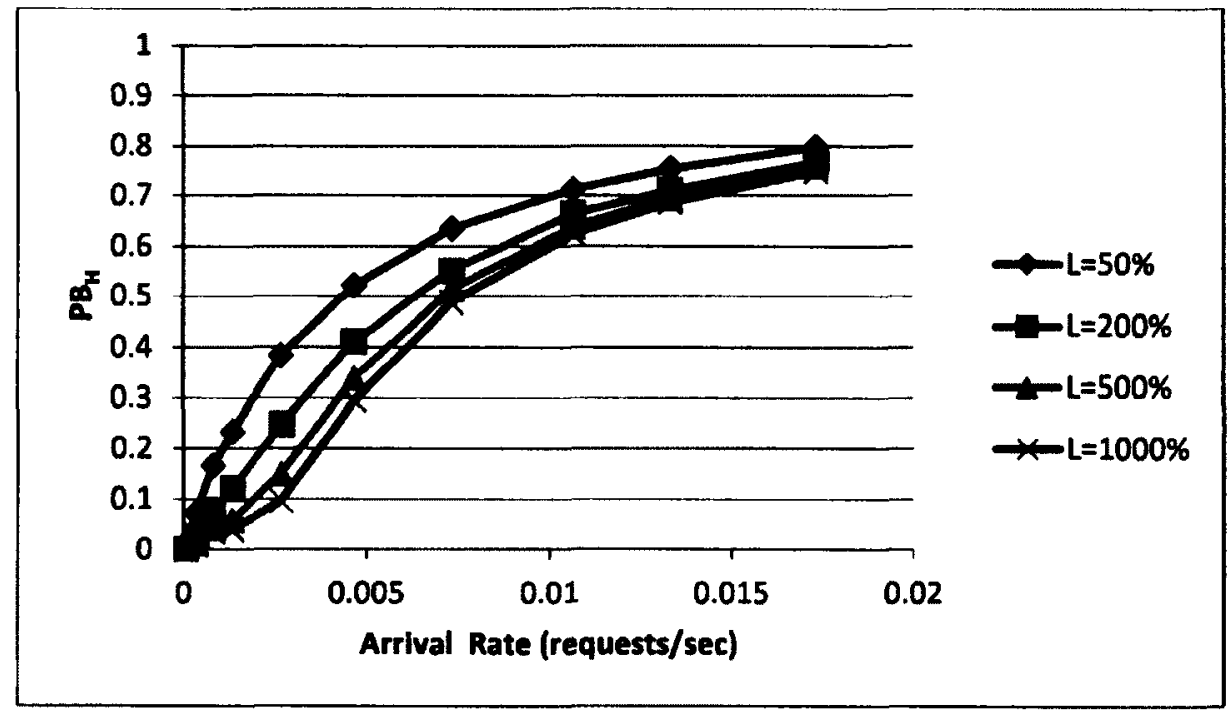

Figure 5-22: Impact of Laxity (L) on the Probability of Blocking High Priority ARs (PB 
The values for probability of blocking low priority requests $\left(\mathrm{PB}_{\mathrm{L}}\right)$ are unaffected by laxity. Thus laxity does not have any significant impact on scheduling low priority requests in the system. The results for $\mathrm{PB}_{\mathrm{L}}$ display a trend similar to the one captured in Figure 5-1 and is included in Appendix A, Figure A-11.

Figure 5-24 shows the average resource utilization due to high priority requests $\left(U_{H}\right)$ for different laxity values. An increase in the number of accepted requests with an increase in laxity, as shown in Figure 5-24, allows resources to run more high priority requests resulting in higher $U_{H}$ values. The average resource utilization due to low priority requests $\left(U_{L}\right)$ displays a trend similar to the one captured in Figure 5-4 and is included in Appendix A, Figure A-12.

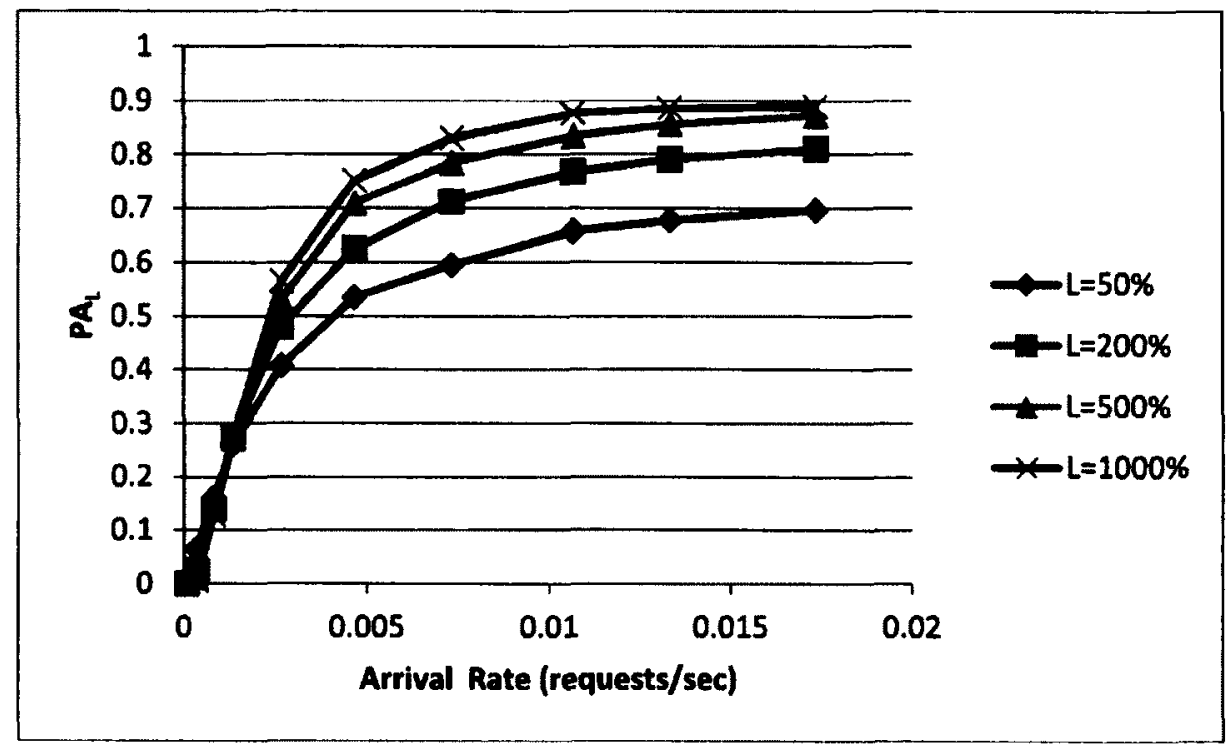

Figure 5-23: Impact of Laxity (L) on the Probability of Aborting Low Priority ARs (PA $\left.A_{L}\right)$

Figure 5-23 shows the probability that a low priority $A R$ is aborted $\left(\mathrm{PA}_{\mathrm{L}}\right)$ for the various laxity values. With an increase in the number of $\mathrm{AR}_{\mathrm{H}}$ requests being accepted as laxity 
increases, the probability of aborting low priority requests also increases. This is confirmed in the figure.

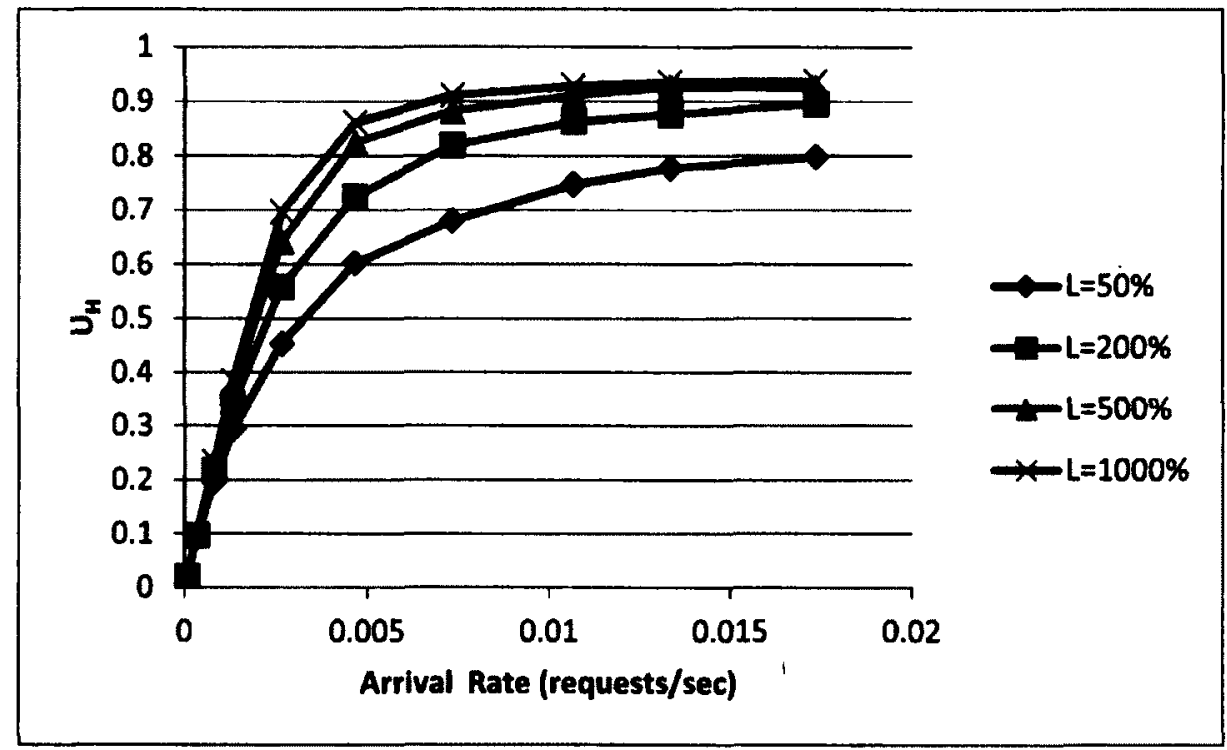

Figure 5-24: Impact of Laxity (L) on the Average Resource Utilization due to High Priority ARs $\left(U_{H}\right)$

\subsubsection{Effect of Padding Time}

The impact of the request's padding time is investigated here. In this set of experiments, the percentage padding is varied to artificially overestimate the request's service time. The amount of time by which the request's service time is overestimated is referred to as padding time. The padding time cannot be greater than the laxity of the request for reasons mentioned in Section 4.5.2.5. A percentage padding of $100 \%$ gives rise to the request's runtime that is overestimated with a padding time that is equal to the mean service time. The idea behind padding is to see if adding additional idle time will reduce the number of requests that are aborted when accommodating higher priority requests and give rise to an improvement in performance. For this experiment the percentage laxity is set to the default value, which is $200 \%$. All other parameters are set to the default values 
(refer to Table 5-1). The arrival rate is varied in the same way as the other experiments with one resource. The percentage padding values are set to $25 \%, 50 \%, 100 \%$, and $200 \%$. When a request finishes execution and padding is used, the padding time gives rise to idle time on the resource. In this set of experiments, after a request completes, the next request in the resource schedule ignores the idle time and begins execution either immediately if possible, or at its earliest start time.

Figure 5-25 shows the probability of blocking high priority requests $\left(\mathrm{PB}_{\mathrm{H}}\right)$ achieved for various padding times. As the padding time increases, the overestimation of the request service time increases leaving less room on the resources to schedule requests. As a result of this, more requests are rejected as shown in the figure, giving rise to a lower number of requests being accepted in the system. $A$ similar trend is observed for $\mathrm{PB}_{\mathrm{L}}$ (see Appendix A, Figure A-13). The improvements on the probability of blocking are magnified in the rate of accepted requests. The overall rate of accepted requests $\left(\mathrm{A}_{\mathrm{T}}\right)$ which includes both low and high priority requests is included in Appendix A, Figure A-14.

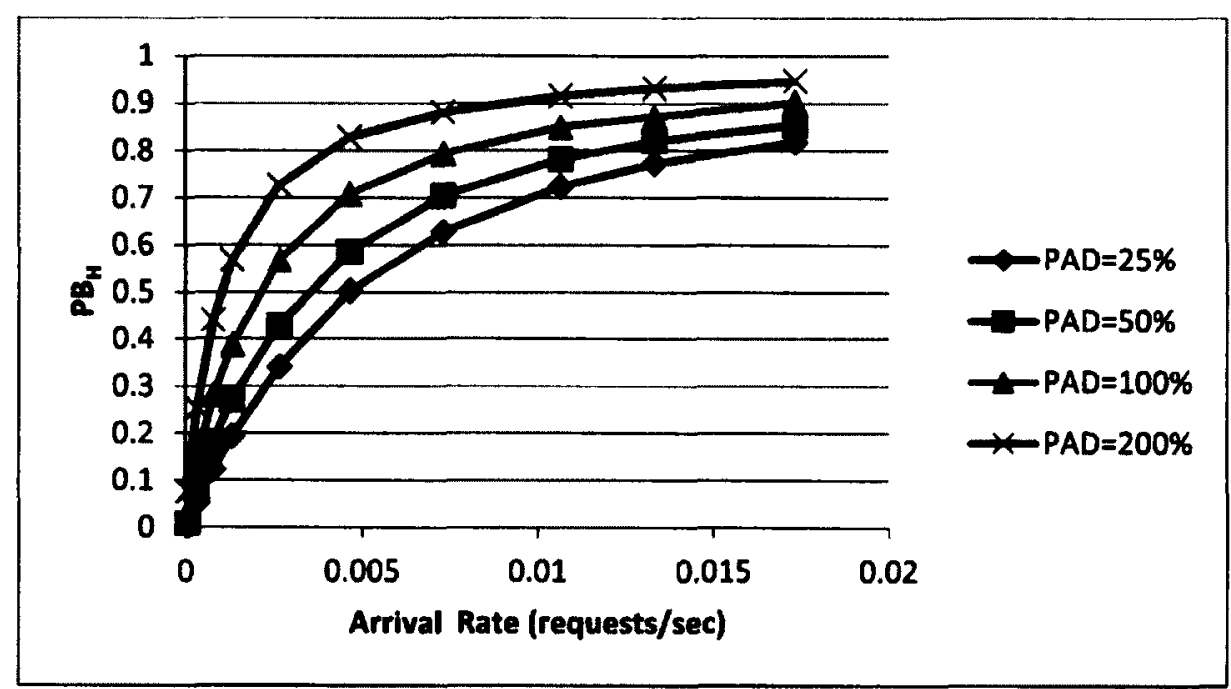

Figure 5-25: Impact of Padding on the Probability of Blocking High Priority ARs (PB 
Figure 5-26 shows the probability that a low priority request is aborted $\left(P A_{L}\right)$ for the various padding times. For a given arrival rate, it is observed that as padding increases, $\mathrm{PA}_{\mathrm{L}}$ also increases. One explanation for this is that since padding artificially increases the service time of both low and high priority $\mathrm{AR}$ requests, the additional time added to the higher priority request's service time causes more requests to be aborted. This is confirmed in the figure.

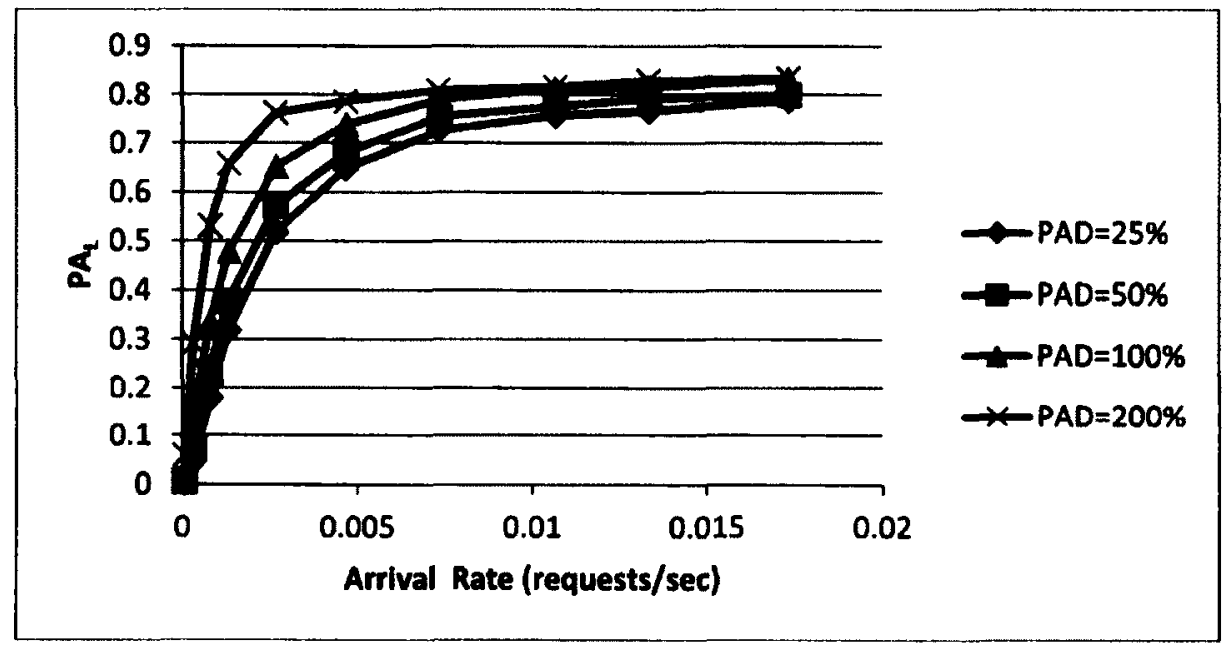

Figure 5-26: Impact of Padding on the Probability of Aborting Low Priority ARs (PA $)$

The average resource utilization due to all requests $\left(U_{T}\right)$ is more important when analysing padding than a breakdown of utilization by priority levels. For this reason, $U_{L}$ and $U_{H}$ are not displayed when analysing padding. Figure 5-27 shows the impact that padding has on $U_{T}$. The results demonstrate that for medium and high arrival rates, lower $\mathrm{U}_{\mathrm{T}}$ values are achieved for higher padding values. Although padding is simply a means of overestimating the request service time, when a request finishes execution, the padding time is converted into idle time on the resource. This along with the fact that more requests are rejected as padding increases, leads to a dramatic decrease in utilization of 
the resources. Because padding only resolves into idle time after AR requests complete, and since $\mathrm{AR}_{H}$ requests cannot pre-empt other $\mathrm{AR}$ requests, padding is not beneficial in a system that does not use pre-emption. Refer to Section 5.3.6 for the impact that padding plays on a system with Emergency requests that require pre-emption. Refer to Appendix A, Figure A-15 for the average resource utilization of high priority request $\left(U_{H}\right)$.

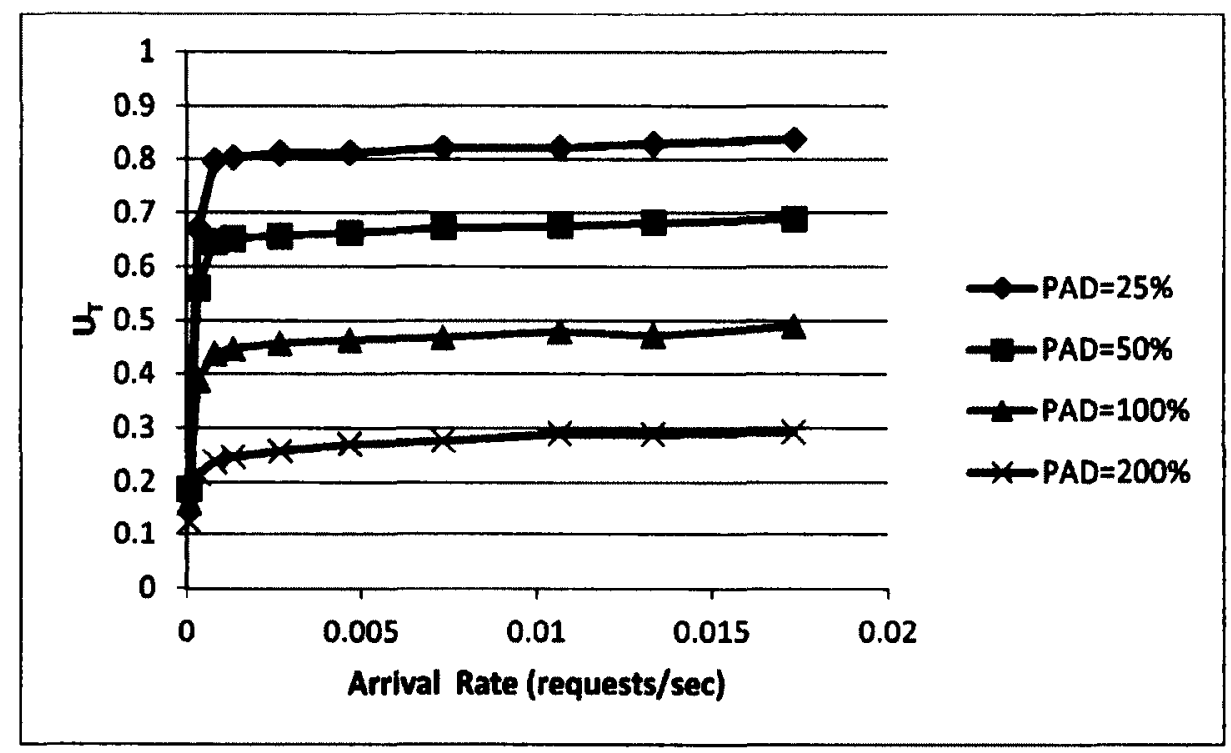

Figure 5-27: Impact of Padding on the Overall Average Resource Utilization $\left(U_{T}\right)$

\subsubsection{Effect of the Proportion of High Priority AR Requests}

This section studies the impact of the proportion of requests with a high priority level $\left(\mathrm{PAR}_{\mathrm{H}}\right)$ on system performance. In this set of experiments, $\mathrm{PAR}_{\mathrm{H}}$ is varied to subject the workload to different combinations of low and high priority requests. Since $\mathrm{PAR}_{H}$ represents the proportion of high priority AR requests, the remainder of requests have a low priority level. Other parameters are set to their default values (see Table 5-1). The arrival rate is varied in the same way as the other experiments with one resource. 
Figure 5-28 shows the $\mathrm{PB}_{\mathrm{H}}$ values obtained for various proportions of high priority requests. As expected, for a given arrival rate, an increasing proportion of high priority requests result in more $\mathrm{AR}_{\mathrm{H}}$ requests being rejected. As the arrival rate increases, so does the contention on the resources resulting in higher $\mathrm{PB}_{\mathrm{H}}$ values. The values for probability of blocking low priority requests $\left(\mathrm{PB}_{\mathrm{L}}\right)$ are unaffected by $\mathrm{PAR}_{\mathrm{H}}$. Thus, the proportion of high priority requests does not seem to have any significant impact on scheduling low priority requests in the system. One explanation for this is that a low priority request is always rejected if the resource schedule is full, as low priority requests cannot abort other requests. The results for $\mathrm{PB}_{\mathrm{L}}$ display a trend similar to the one captured in Figure 5-1 and is included in Appendix A, Figure A-16.

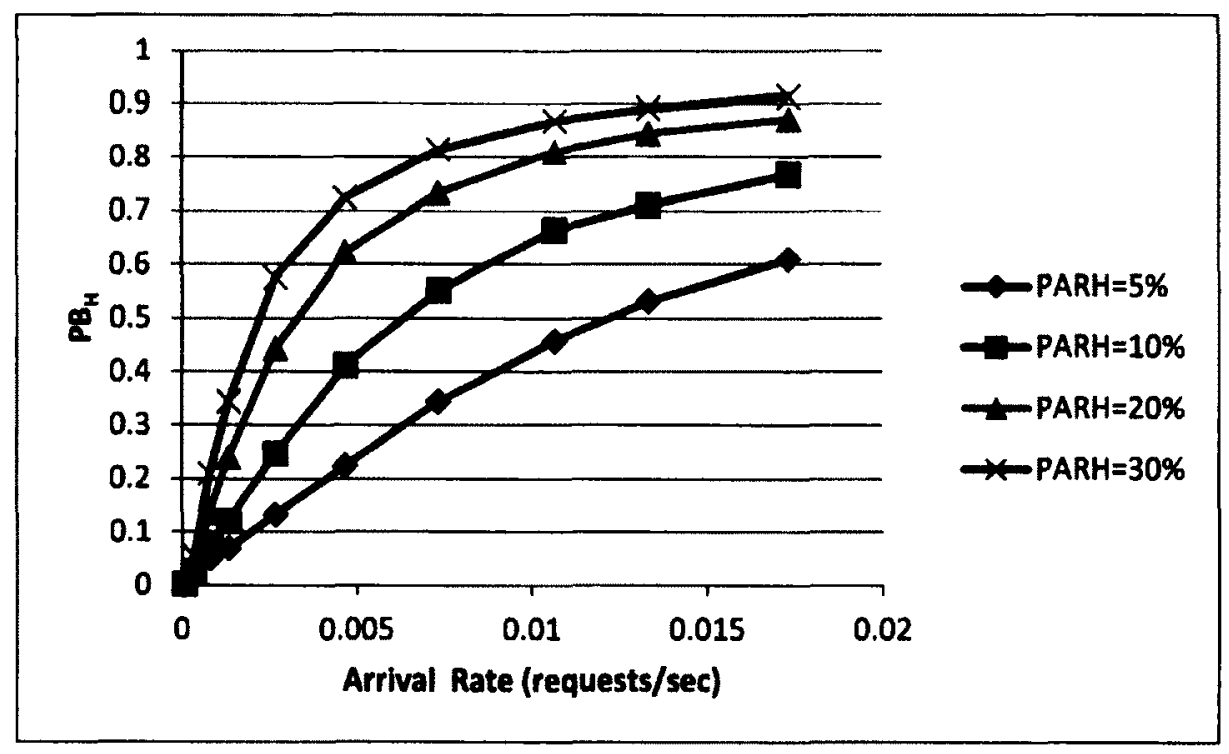

Figure 5-28: Impact of the Proportion of High Priority ARs $\left(\mathrm{PAR}_{H}\right)$ on the Probability of Blocking High Priority ARs $\left(\mathbf{P B}_{\mathrm{H}}\right)$

Figure 5-29 displays the probability that a low priority request is aborted $\left(\mathrm{PA}_{\mathrm{L}}\right)$ for the various $\mathrm{PAR}_{\mathrm{H}}$ values. As expected, the figure shows that as more high priority requests are submitted on system there is a greater chance that low priority requests are aborted. In 
addition, with an increase in the proportion of high priority requests the average resource utilization due to high priority requests $\left(U_{H}\right)$ increases. This is observed in Figure 5-30. The average resource utilization due to low priority requests $\left(\mathrm{U}_{\mathrm{L}}\right)$ displays a trend similar to the one captured in Figure 5-4 and is included in Appendix A, Figure A-17.

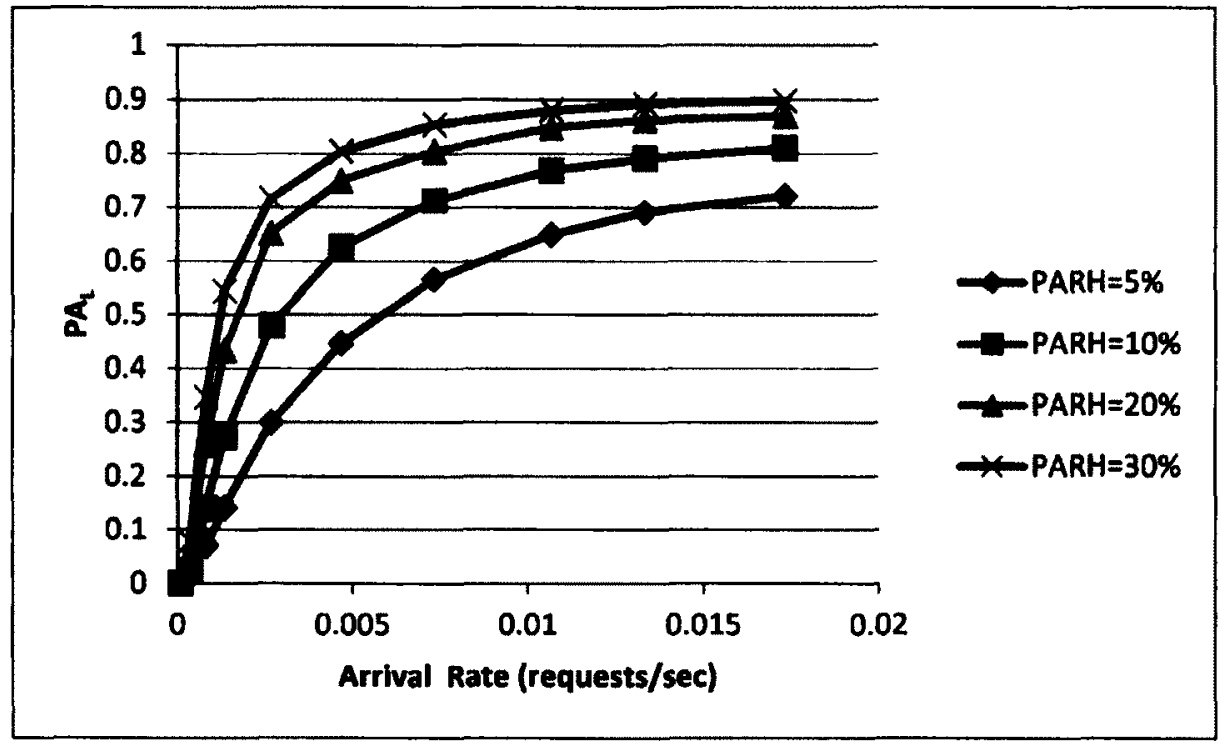

Figure 5-29: Impact of the Proportion of High Priority ARs (PAR $\left(R_{H}\right)$ on the Probability of Aborting Low Priority ARs (PA $)$

\subsection{Performance of a System with Emergencies}

The performance analysis of a system without Emergency requests is presented in Section 5.2. This section discusses the performance of a system consisting of a workload containing $\mathrm{AR}$ requests having various priority levels as well as Emergency requests.

\subsubsection{Effect of the Pre-emption Policy}

This section studies the impact of the pre-emption policies on the performance of the system. The pre-emption policies are used by the broker when multiple resources are available to determine an appropriate resource to execute an Emergency request. The pre- 
emption policies used in these experiments include Largest Slack (LS-P), Greatest Service Time (GS-P), Shortest Time (ST-P) and Next Resource (NR-P). Refer to Section 3.3.3 for a description of the mentioned pre-emption policies. In order to avoid confusion with abortion policies, a "-P" is appended to the acronym of the pre-emption policy. To determine the performance of the pre-emption policies under different loads, the arrival rates of the workload is varied from low to high until very high system utilization and probability of blocking values are achieved. A resource pool of 25 resources is used for the experiments in this section. All other parameters are set to their default values (see Table 5-1).

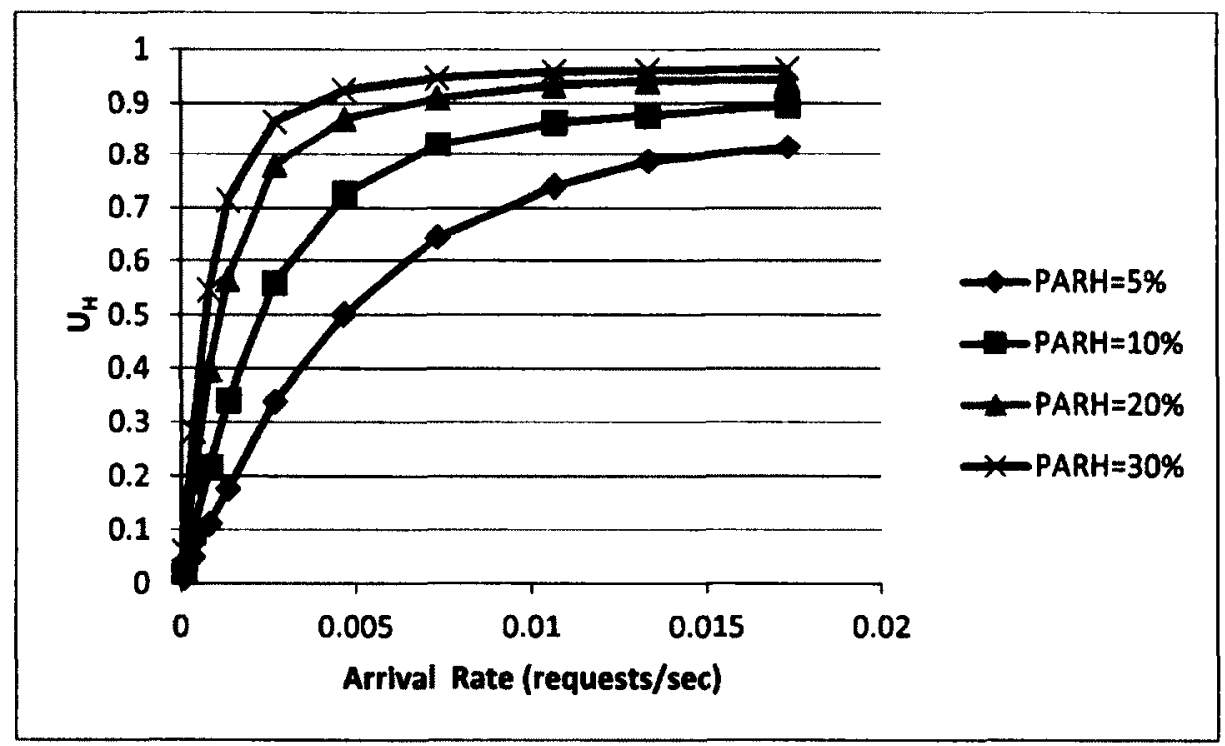

Figure 5-30: Impact of the Proportion of High Priority ARs (PAR $)$ on the Average Resource Utilization due to High Priority ARs $\left(U_{H}\right)$

For the results presented in this section, the broker is unaware of the Emergency request's service time when determining a resource for execution. Section 5.3.3 investigates the impact on the performance of the system when the Emergency request's service time is known. For the experiments discussed in this section, when an Emergency request is 
scheduled for execution, the job currently running is pre-empted and put into the ready queue. When the emergency finishes, the pre-empted job resumes execution provided it can still meet its deadline, otherwise it is aborted from the system and the next request in the resource schedule that can meet its deadline begins execution.

The default abortion policy used for the simulations in this section is NA-A. For any of the pre-emption policies, the values for the probability of blocking low priority requests $\left(\mathrm{PB}_{\mathrm{L}}\right)$ for a given arrival rate are statistically comparable to the $\mathrm{PB}_{\mathrm{L}}$ values achieved for NA-A without pre-emption shown in Figure 5-1. This indicates that the occurrence of emergencies do not affect whether a low priority request gets blocked. This is because the rejection of an $A R_{L}$ request occurs before the occurrence of an emergency and thus ${ } B_{L}$ is not affected significantly by Emergency requests. The same is observed in the case of $\mathrm{PB}_{\mathrm{H}}$. For this reason, the $\mathrm{PB}_{\mathrm{L}}$ and $\mathrm{PB}_{\mathrm{H}}$ results for the various pre-emption policies is included in Appendix B, Figure B-1 and Figure B-2 respectively. The analysis for $\mathrm{PB}_{\mathrm{L}}$ and $\mathrm{PB}_{\mathrm{H}}$ is the same as in Section 5.2.1. Since Emergency requests are always accepted the probability of blocking these requests is zero. Because Emergencies do not seem to have an impact on the probability of blocking (both $\mathrm{PB}_{\mathrm{L}}$ and $\mathrm{PB}_{\mathrm{H}}$ ), these performance metrics will not be reported for the remainder of this chapter. As well, since the rate of accepted requests $(A)$ is directly computed from $P B, A_{L}$ and $A_{H}$ will also not be reported for the rest of this chapter.

Figure 5-31 displays the average resource utilization due to Emergency requests $\left(U_{E}\right)$ for the various pre-emption policies. For a given arrival rate, all of the pre-emption policies have statistically comparable $U_{E}$ values. Since Emergency requests are always accepted, as the arrival rate increases, Emergency requests arrive on the system faster and the 
average resource utilization increases for any given pre-emption policy. This is confirmed in the figure.

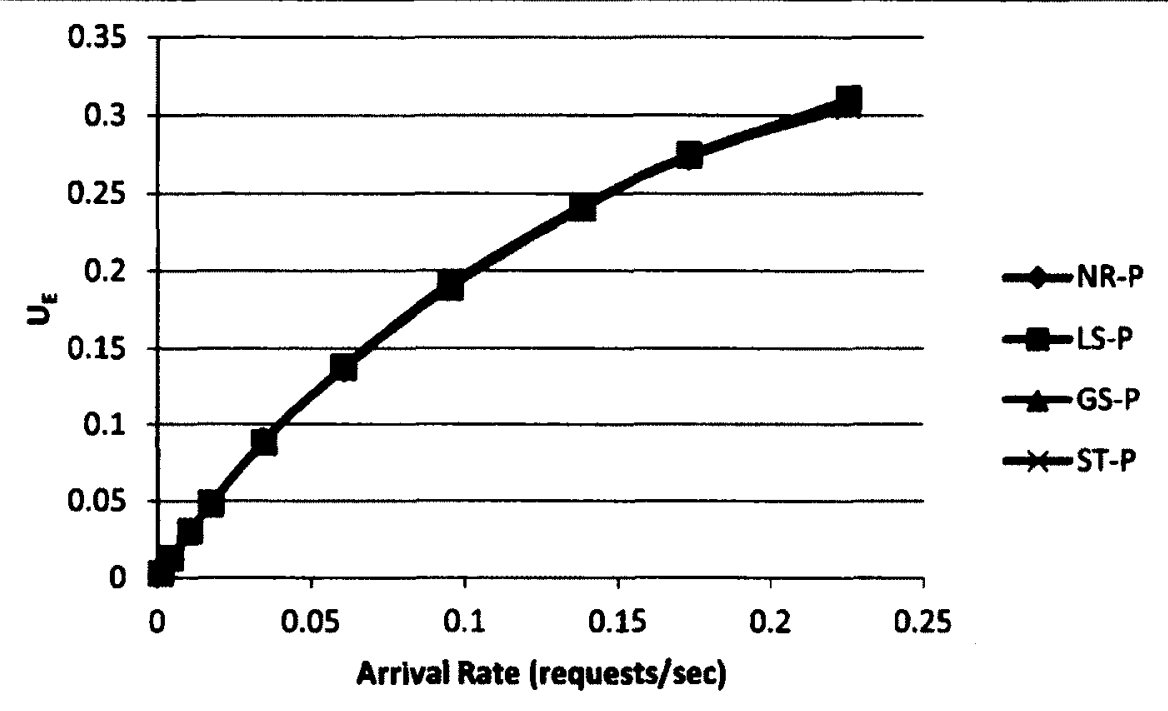

Figure 5-31: Impact of the Pre-emption Policies on the Average Resource Utllization due to Emergencies $\left(U_{E}\right)$

Figure 5-32 shows the average resource utilization due to high priority AR requests $\left(U_{H}\right)$ for the different pre-emption policies. At low arrival rates, all of the pre-emption policies achieved statistically comparable $U_{H}$ values as there is little contention on the resources. For medium and high arrival rates, the $U_{H}$ values for all of the pre-emption policies at a given arrival rate seem to be statistically comparable with one another.

Figure 5-33 shows the average resource utilization for low priority AR requests $\left(U_{L}\right)$ for the different pre-emption policies. As the majority of requests in the system are low priority ARs, a high utilization for low priority requests is produced at lower arrival rates where the contention is low. At medium and high arrival rates, the contention on the resources increases. As more high priority requests are accepted into the system, more 
low priority AR requests are aborted resulting in a lower $U_{L}$, as observed in Figure 5-33. For a given arrival rate, the $U_{L}$ values for all of the pre-emption policies are statistically comparable to one another.

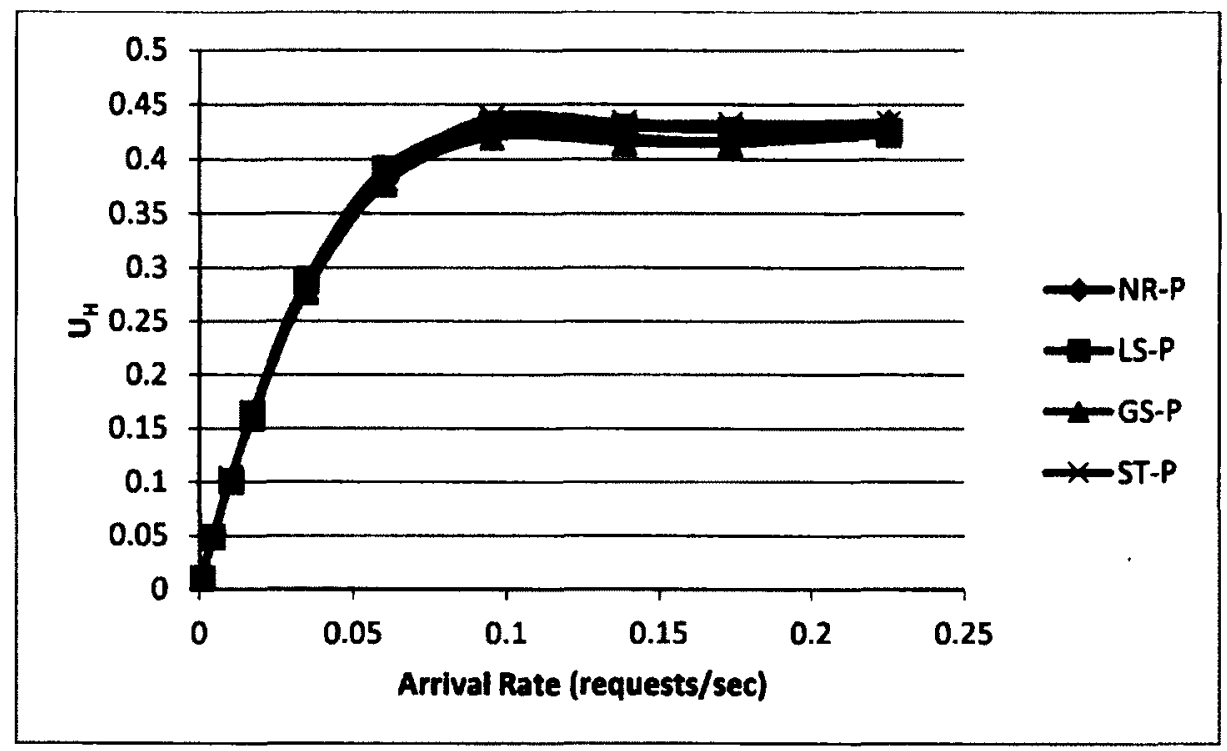

Figure 5-32: Impact of the Pre-emption Policies on the Average Resource Utilization due to High Priority ARs $\left(\mathrm{U}_{\mathrm{H}}\right)$

The average resource utilization due to all requests $\left(U_{T}\right)$ is the overall utilization of the system. Here, $\mathrm{U}_{\mathrm{T}}$ is computed as the summation of $\mathrm{U}_{\mathrm{L}}, \mathrm{U}_{\mathrm{H}}$, and $\mathrm{U}_{\mathrm{E}}$ for a given arrival rate and is included in Appendix B, Figure B-3. Since $U_{T}$ can be calculated from $U_{L}, U_{H}$, and $\mathrm{U}_{\mathrm{E}}$ it will not be reported in the following sections.

Figure 5-34 shows the probability of an AR request being aborted because of an emergency (PA-E) for the various pre-emption policies. For all of the pre-emption policies, as the arrival rate increases, the PA-E values also increase. One explanation for this is that more AR requests will be aborted because Emergency requests utilize more resources as the arrival rate increases. As NR-P only looks at one resource, while the 
other pre-emption policies iteratively look at all of the available resources when selecting a resource to execute the Emergency request, NR-P produces the highest PA-E values. Although NR-P produces PA-E values that are higher than the other pre-emption policies, the PA-E values are still close to the values achieved for the other pre-emption policies for a given arrival rate. In terms of PA-E, GS-P performs slightly better than the other pre-emption policies for medium and high arrival rates.

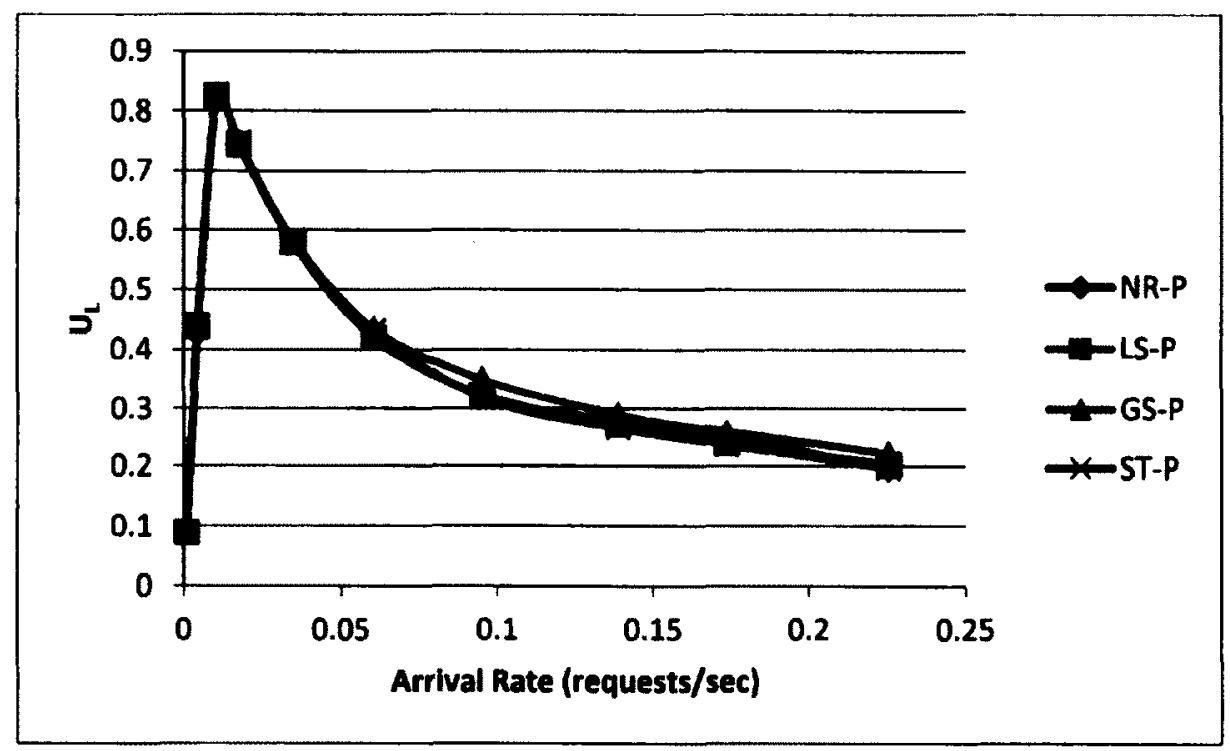

Figure 5-33: Impact of the Pre-emption Policies on the Average Resource Utilization due to Low Priority ARs $\left(\mathrm{U}_{\mathrm{L}}\right)$

Other pre-emption policies using different metrics for determining the matchmaking of Emergency requests have been simulated, but are not presented here as they did not produce significantly different results. These include selecting the resource with the greatest interval, and selecting the resource with the fewest number of scheduled jobs. For the greatest interval, the interval is computed as the difference between the arrival time for the Emergency request and the start time of the next request scheduled to run. 


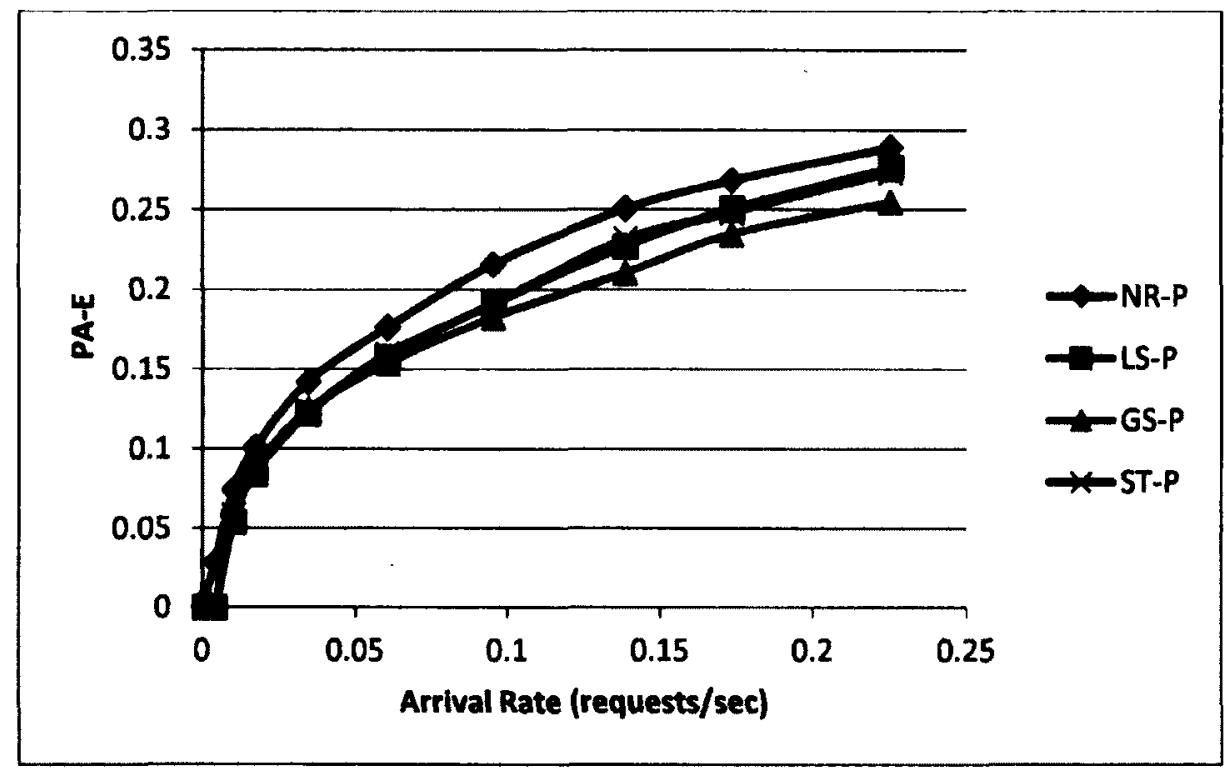

Figure 5-34: Impact of the Pre-emption Policies on the Probability of Aborting ARs because of Emergencies (PA-E)

In terms of utilization, the values produced by NR-P are statistically comparable to the other pre-emption policies for a given arrival rate. When considering system overhead, it seems that NR-P is the most attractive pre-emption policy as it requires the lowest overhead since it only looks at one resource, while the other pre-emption policies iteratively look at all of the available resources when selecting a resource to execute the emergency. Although NR-P has the lowest overhead, it comes with slightly higher PA-E values for medium and high arrival rates in comparison to the other pre-emption policies. If the probability of aborting a request due to an emergency is the main concern, GS-P seems to be the most attractive pre-emption policy as it produces the lowest PA-E values at medium and high arrival rates. When taking everything into consideration, it is recommended to use NR-P because although it results in slightly higher PA-E values, the results are still comparable to the values of the other policies but the overhead required in 
analysing the resources is greatly reduced. For this reason, NR-P is used as the default pre-emption policy throughout the remainder of the thesis.

\subsubsection{Effect on the Number of Resources}

This set of experiments investigates the impact that Emergency requests have on the resource pool. Apart from Emergency requests, the workload consists of AR requests with multiple priorities. As in Section 5.2, the resource pool is set to 1,5 , and 10 resources. When dealing with Emergency requests, the broker determines the resource to execute the emergency based on the pre-emption policy, in this case NR-P which is outlined in Section 3.3.3.1. Increasing the number of resources in the resource pool allows the broker more options to schedule the Emergency request. All other parameters are set to their default values (see Table 5-1).

Figure 5-35 shows the impact that the number of resources in the resource pool has on the probability of an AR request (both low and high priority) being aborted because of an Emergency request (PA-E). The figure demonstrates that for a given arrival rate, as more resources become available the likelihood of an AR request becoming aborted because of an emergency decreases. This is a result of an increase in flexibility when scheduling requests because more room becomes available to accommodate the emergency. As the number of resources increases, the contention on scheduling a request decreases resulting in a greater chance of $A R$ requests being accepted $\left(A_{T}\right)$, as seen in Appendix $B$, Figure $B-$ 4. 


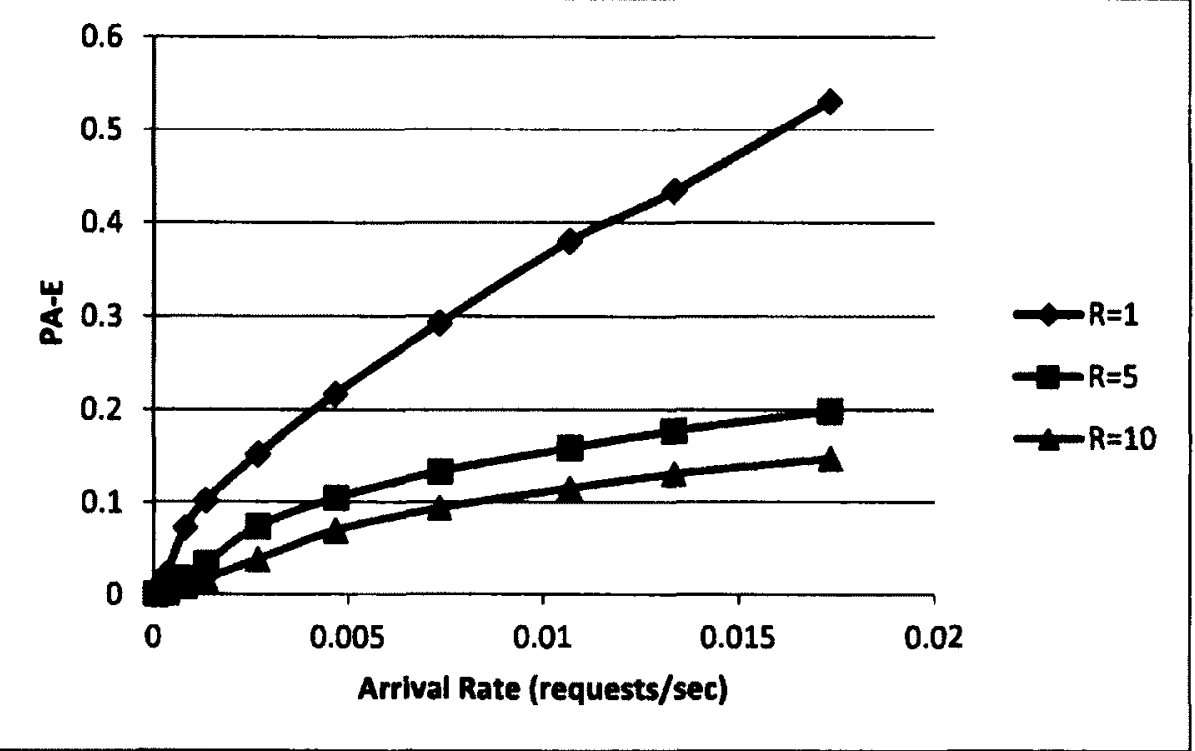

Figure 5-35: Impact of the Number of Resources (R) on the Probability of Aborting Low Priority ARs because of Emergencles (PA-E)

Figure 5-36 shows the impact of the number of resources in the resource pool on the average resource utilization values due to low priority AR requests $\left(U_{L}\right)$. As seen in the figure, at very low arrival rates there is little contention and the requests can be handled by one resource. As the majority of requests in the system are low priority ARs, a high utilization for low priority requests is produced at lower arrival rates where the contention is low. As the arrival rate increases, the contention on the resources also increases causing more requests to be rejected, as well as aborted by higher priority requests. Thus, resulting in a lower $U_{L}$. As seen in the figure for medium and high arrival rates, as the number of resources in the resource pool increases, there is less contention on the resources and more $A R_{L}$ requests can be serviced resulting in a higher utilization of low priority ARs at a given arrival rate. 


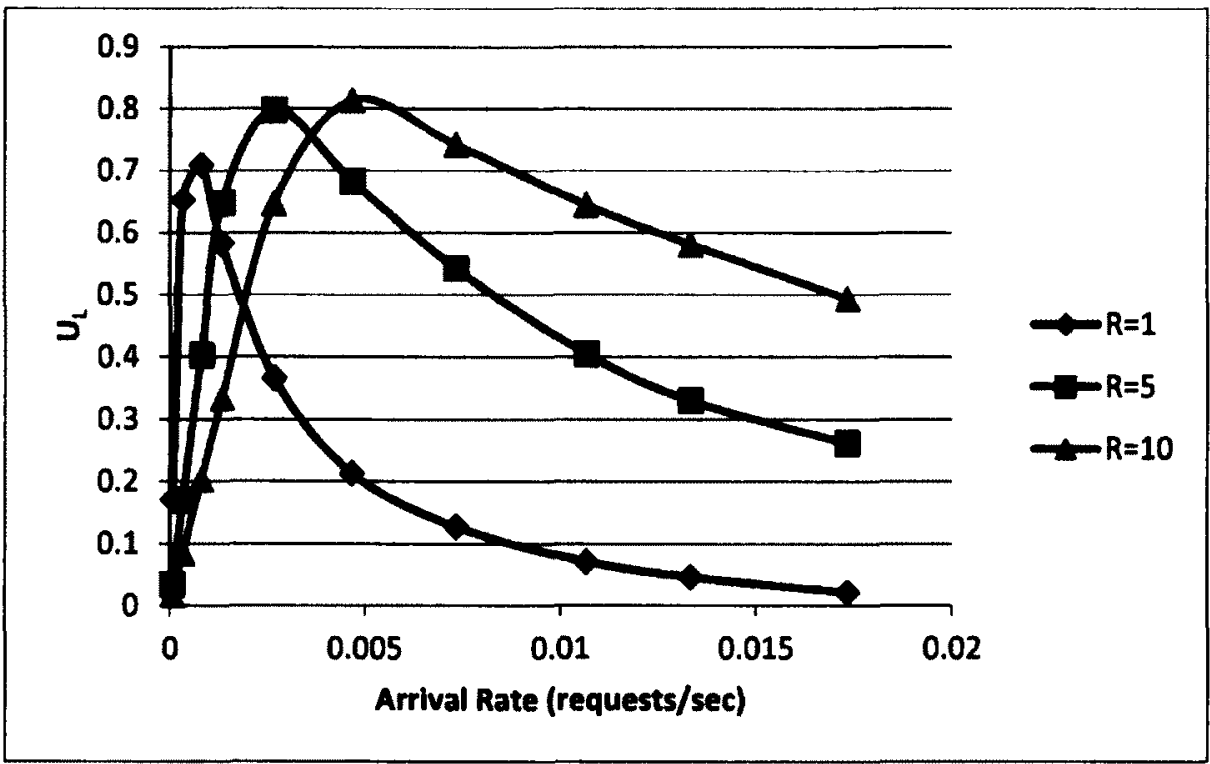

Figure 5-36: Impact of the Number of Resources (R) on the Average Resource Utilization due to Low Priority ARs $\left(U_{L}\right)$

The average resource utilization values for $\mathrm{AR}_{H}$ requests are shown in Figure 5-37. It can be seen that for one resource the $U_{H}$ values increase until a medium level of contention is achieved. In this case, at a medium arrival rate. Arrival rates beyond this point result in $\mathrm{U}_{\mathrm{H}}$ values decreasing because the contention on the resources increases and since Emergency requests have the highest priority, they are accommodated by aborting more $A R_{H}$ requests. Since increasing the number of resources in the resource pool reduces the contention, the $\mathrm{U}_{\mathrm{H}}$ values for 5 and 10 resources increase with the arrival rate, as opposed to the one resource case. This is because for the arrival rates shown, the level of contention required to cause a decrease in $\mathrm{U}_{\mathrm{H}}$ is not yet achieved.

Figure 5-38 shows the average resource utilization due to Emergency requests $\left(U_{E}\right)$ achieved for different $\mathrm{R}$ values. Although the same number of Emergency requests execute for all number of resources, as the number of resources increase, the work gets 
divided among more resources resulting in lower $\mathrm{U}_{\mathrm{E}}$ values for a given arrival rate. This is confirmed in the figure.

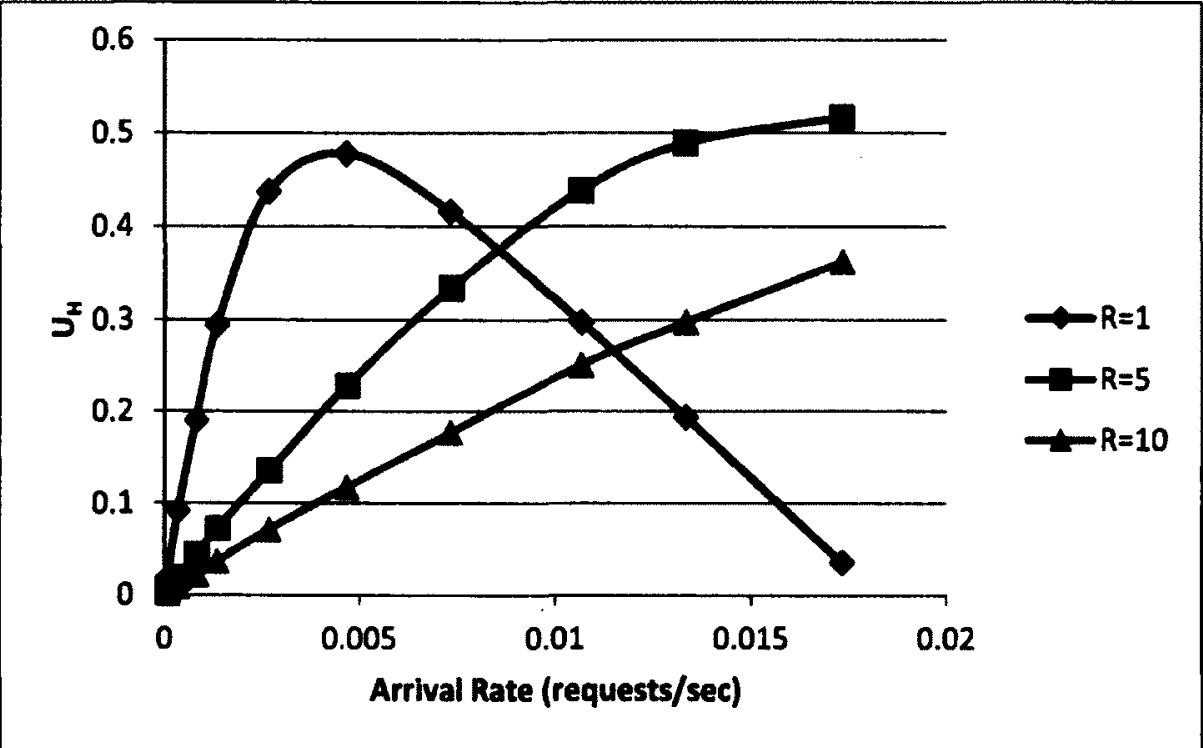

Figure 5-37: Impact of the Number of Resources (R) on the Average Resource Utilization due to High Priority ARs $\left(\mathbf{U}_{\mathbf{H}}\right)$

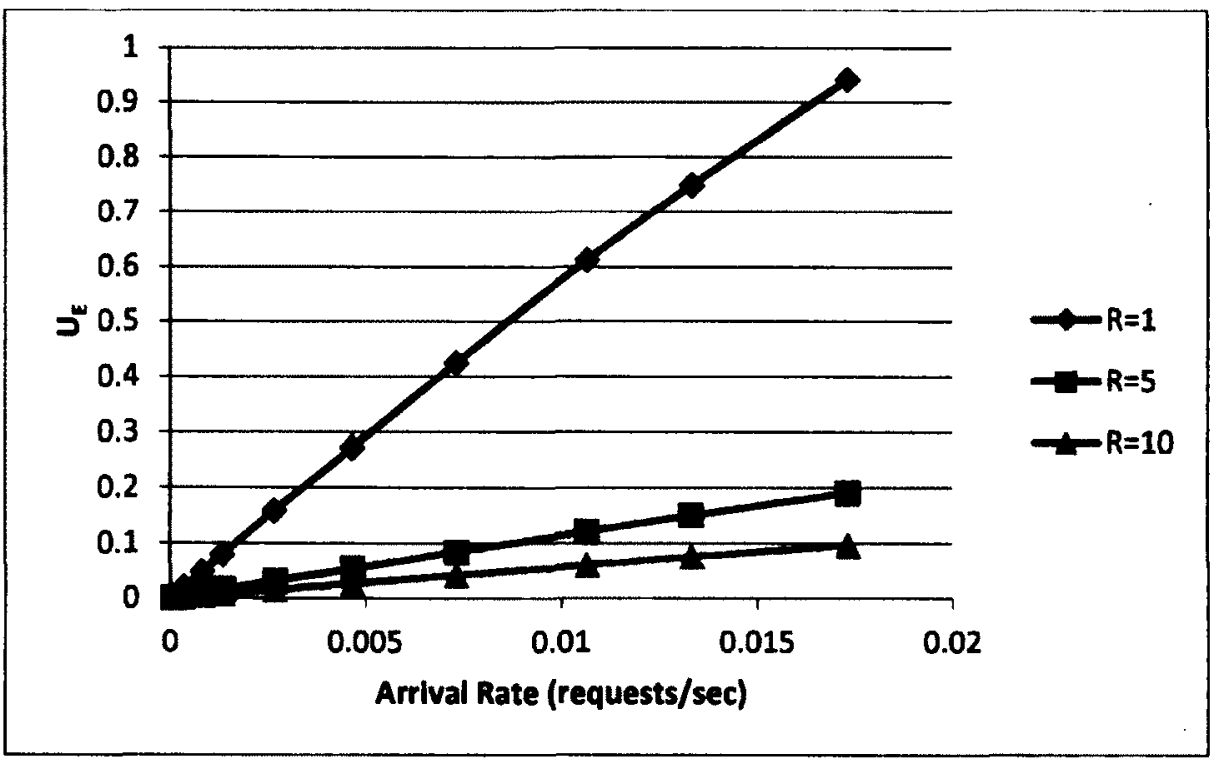

Figure 5-38: Impact of the Number of Resources (R) on the Average Resource Utilization due to Emergencies $\left(\mathbf{U}_{\mathbf{E}}\right)$ 


\subsubsection{Effect of the Knowledge of the Emergency Service Time}

This section presents the impact of the broker knowing the Emergency request's service time and using it when selecting a resource for its execution. Handling emergencies with unknown service times is discussed in Section 3.3.2. When an emergency is scheduled for execution and its service time is unknown, the job currently running is pre-empted. When the emergency finishes, the pre-empted job resumes provided it can still meet its deadline. Otherwise it is aborted from the system and the next request in the resource schedule that can meet its deadline begins execution. In the case where the emergency service times are known, when an Emergency request is scheduled, the job currently running is pre-empted as in the case where the emergency service time is unknown. However, since the emergency service time is known in this case, the scheduler can determine if the pre-empted job and other requests in the resource schedule that overlap with the emergency can still meet their deadline after the Emergency request completes. If they can still meet their deadlines, they are allowed to wait for the emergency to finish, and then resume execution. If it is deemed that the pre-empted jobs can no longer meet their deadlines on this resource after the emergency finishes, they are sent to the broker for reallocation. The jobs are aborted from the system if they cannot be reallocated. Provided the job can be reallocated on another resource, the job will either resume or restart depending on the reallocation type. The impact on the reallocation type is explored in the following section. In the set of experiments for this section, the system uses preemptive resume with a resource pool of 25 resources. All other parameters are kept at their default values (refer to Table 5-1). 
The simulation results show that knowing the Emergency request's service time at the time of arrival does not have any significant impact on PA-E, $\mathrm{U}_{\mathrm{L}}, \mathrm{U}_{\mathrm{H}}$, and $\mathrm{U}_{\mathrm{E}}$ observed at a given arrival rate. Thus, only the figure for PA-E is presented (see Figure 5-39). The graph when the emergency service time is unknown overlaps with the graph when the emergency service time is known. Each of the other performance metrics listed have comparable values for a given arrival rate as that presented for the NR-P graphs in Section 5.3.1. Indicated by the log files collected during the simulation runs, there is an extremely low number of requests that actually get reallocated when the emergency service time is known. As a result, the performance metrics are not significantly impacted. Thus, it seems that there is no significant differences with the case where the emergency service time is unknown.

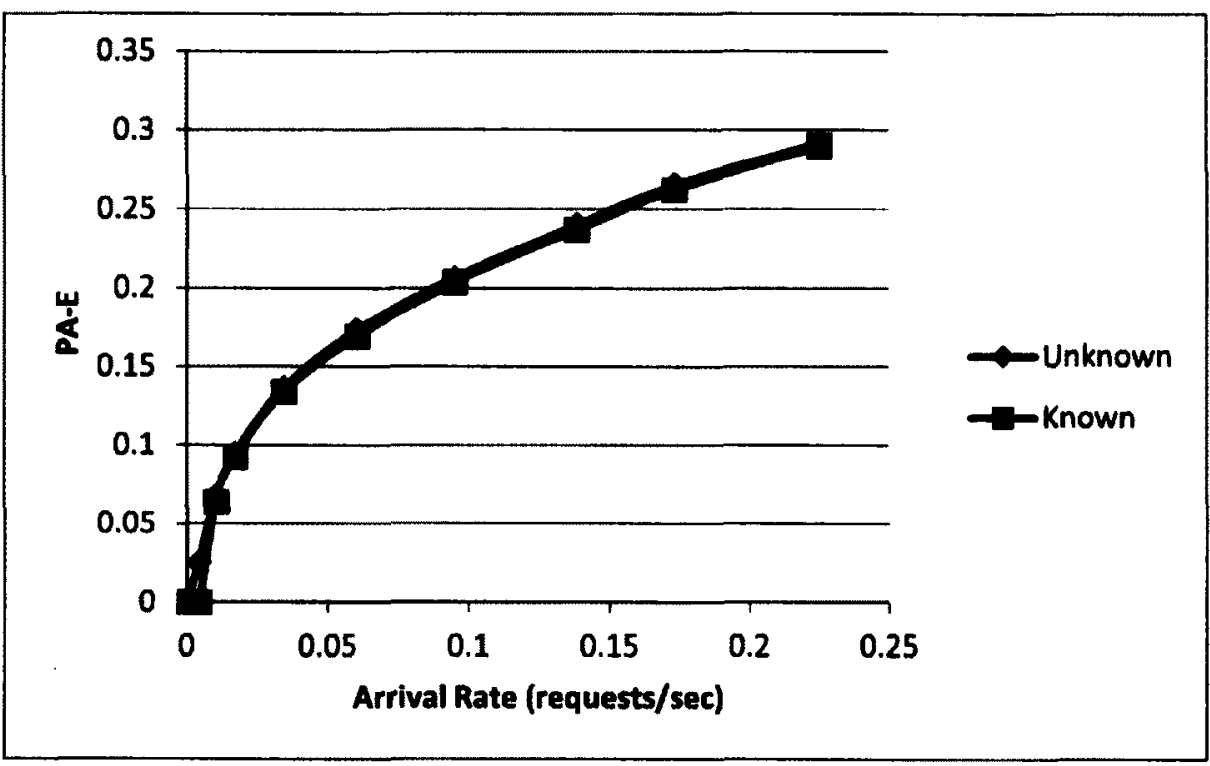

Figure 5-39: Impact of Knowing the Emergency Service Time on the Probability of Aborting ARs becuase of Emergencles (PA-E) 


\subsubsection{Effect of Reallocation Type}

This section studies the impact of the reallocation type on system performance. In this set of experiments, the emergency service time must be known for reasons mentioned in Section 5.3.3. The reallocation type is important when the pre-empted job is reallocated on another resource where the job may be resumed or restarted. When using pre-emptive resume, the request will commence execution from where it left off on the previous resource. Pre-emptive restart will restart the request on the new resource. In this set of experiments the resource pool consists of 25 resources. All other parameters are held at their default values (refer to Table 5-1).

The simulation results show that when a request is reallocated after being pre-empted, whether the request was resumed or restarted on another resource did not have any significant impact on PA-E, $U_{L}, U_{H}$, and $U_{E}$ observed for a given arrival rate. Thus, only the figure for PA-E is presented (see Figure 5-40). The graph for pre-emptive restart overlaps with that for pre-emptive resume. Each of the other performance metrics listed have comparable values for a given arrival rate as that presented for the NR-P graphs in Section 5.3.1. As indicated by the log files collected during simulation runs, pre-emptive resume and pre-emptive restart do not produce different results because they give rise to a comparable number of requests that are reallocated. In addition, only a very small number of requests actually become reallocated once they are aborted, regardless of whether pre-emptive resume or pre-emptive restart is used. The proportion of requests that are reallocated for each priority level are also similar. 


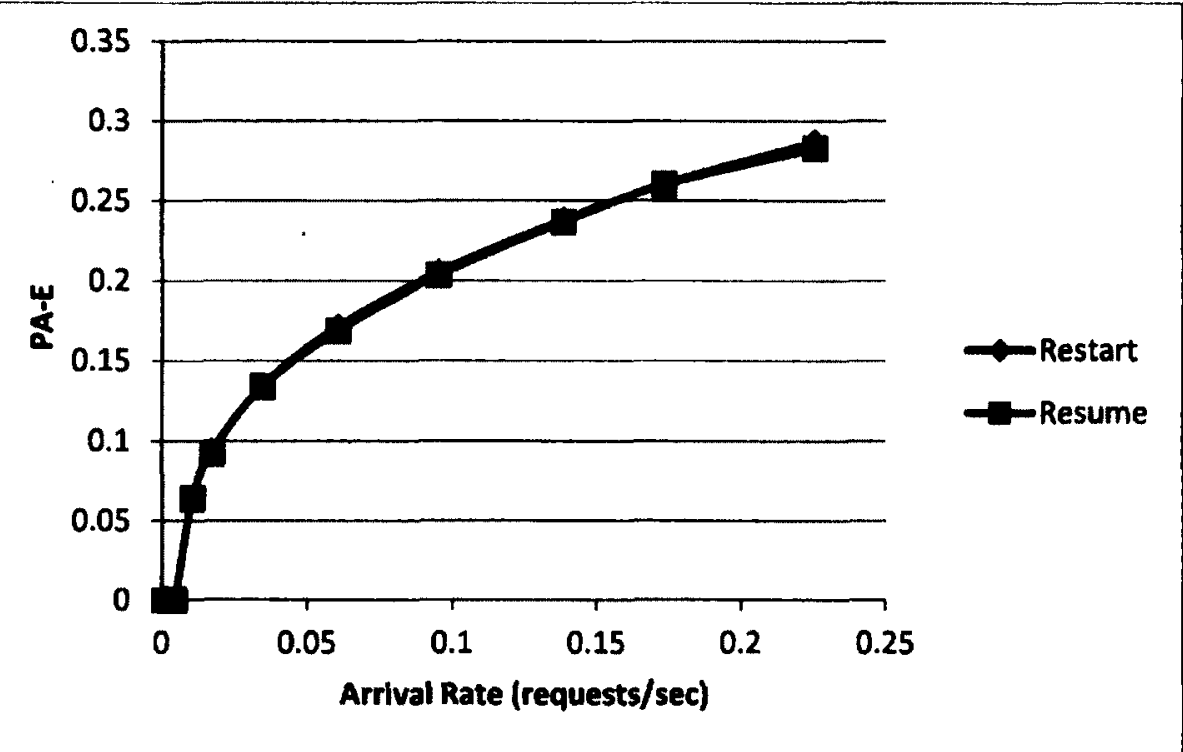

Figure 5-40: Impact of the Reallocation Type on the Probability of Aborting ARs because of Emergencies (PA-E)

\subsubsection{Effect of Emergency Service Time}

This section presents the impact of the mean and variability for the service time of Emergency requests (ES) on system performance. As in Section 5.2.4, two experiments are required when investigating the service time of Emergency requests. The first one looks at the mean ES on performance, while the other focuses on the impact of the variability factor. Refer to Section 5.2 .4 for how the lower and upper bounds of the uniform distribution for the service times are computed. The arrival rate is varied in the same way as in the other experiments with one resource. All other parameters are held at their default values (refer to Table 5-1).

\subsubsection{Effect of the Emergency Service Time Variability Factor}

In this set of experiments for $\mathrm{ES}$, the variability factor, denoted as $\mathrm{V}_{\mathrm{f}, \mathrm{E}}$, is varied to study system performance. The variability factor is used to adjust the upper and lower bound of the uniform distribution when generating the service times for Emergency requests. 
Throughout these experiments the mean ES remains constant at 20 minutes. Similar to Section 5.2.4.1, $\mathrm{V}_{\mathrm{f}, \mathrm{E}}$ is set to three distinct levels of variability including $0.05,0.5$, and 0.95 resulting in the variability of ES with the uniform distribution of (19 minutes and 21 minutes), (10 minutes and 30 minutes), and (1 minutes to 39 minutes), respectively.

The simulation results show that the variability factor for Emergency requests does not have a significant impact on PA-E, $U_{L}, U_{H}$, and $U_{E}$ observed for a given arrival rate. Thus, only the figure for PA-E is presented (see Figure 5-41). The graphs for all three levels of variability overlap with each other. Each of the other performance metrics listed have comparable values for a given arrival rate as that presented for the $R=1$ graphs in Section 5.3.2. Since emergencies are always accepted and a uniform distribution is used with the same mean emergency service time, the cumulative amount of time that the Emergency requests spend executing seems to be the same regardless of the variability factor.

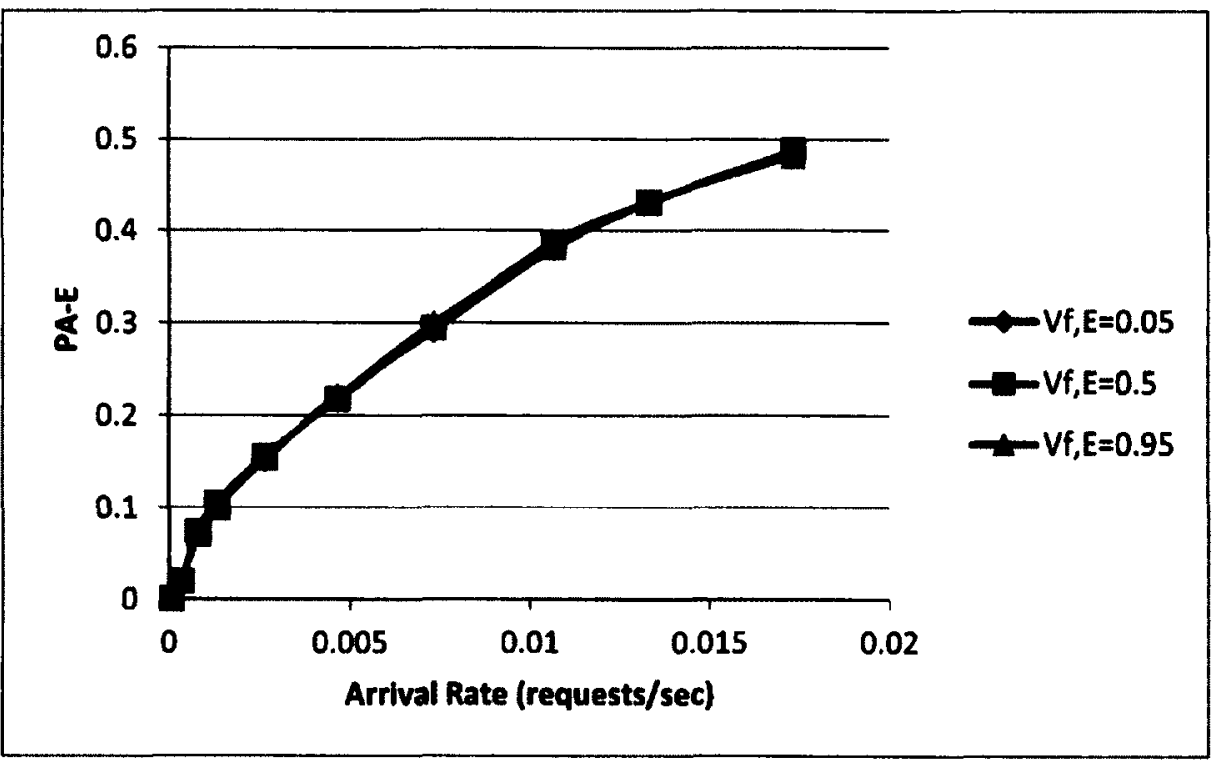

Figure 5-41: Impact of the Variability Factor for Emergencles $\left(V_{r, \Sigma}\right)$ on the Probability of Aborting ARs because of Emergencies (PA-E) 


\subsubsection{Effect of Mean Emergency Service Time}

This set of experiments investigates the impact that the mean service time of Emergency requests has on system performance. Three levels of mean service time are studied while keeping the variability factor constant at its default value of 0.8 . The low level mean service time is set to 10 minutes thereby uniformly generating Emergency requests with service times between 12 and 18 minutes. The medium level mean service time is set to 25 minutes resulting in Emergency requests being generated with a service time between 5 and 45 minutes. The high-level mean service time is set to 50 minutes thereby generating emergency requests between 10 and 90 minutes.

Figure 5-42 shows the average resource utilization due to Emergency requests $\left(U_{E}\right)$ for different mean emergency service times. As emergency service times increase, more time is required to service the request, thus resulting in an increase in $U_{E}$ for a given arrival rate. This is confirmed in the figure.

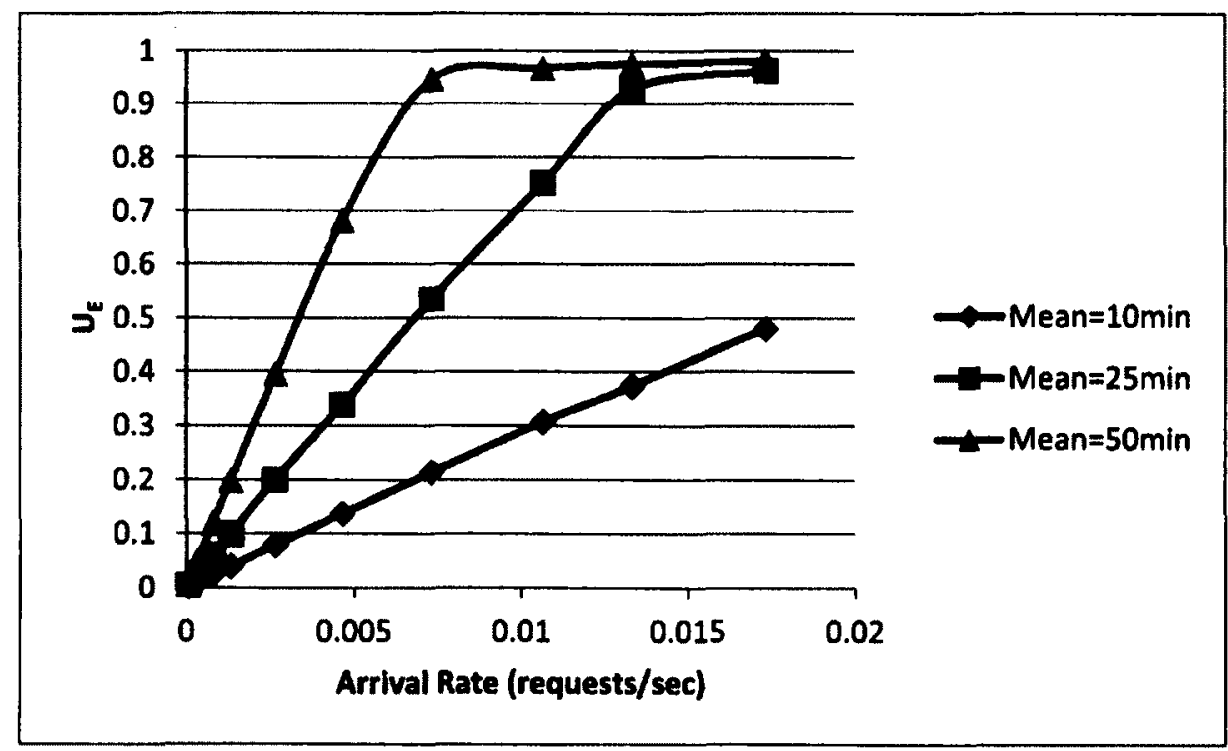

Flgure 5-42: Impact of the Mean Emergency Service Time on the Average Resource Utilization due to Emergencies $\left(U_{E}\right)$ 
Figure 5-43 shows the average resource utilization due to high priority AR requests $\left(U_{H}\right)$ for the different emergency mean service times. At low arrival rates, the $U_{H}$ values are statistically comparable for the different mean service times as there is low contention on the resources. For medium and high arrival rates, as the emergency mean service time increases the Emergency requests execute on the resources longer. As a result, more AR requests (both low and high priority) are aborted with an increasing mean ES giving rise to lower $U_{H}$ values as seen in the figure. The average resource utilization due to low priority AR requests $\left(U_{L}\right)$ can be explained similarly to $U_{H}$. As the trend for the $U_{L}$ graphs is similar to Figure 5-33, the $U_{L}$ results are included in Appendix B, Figure B-5.

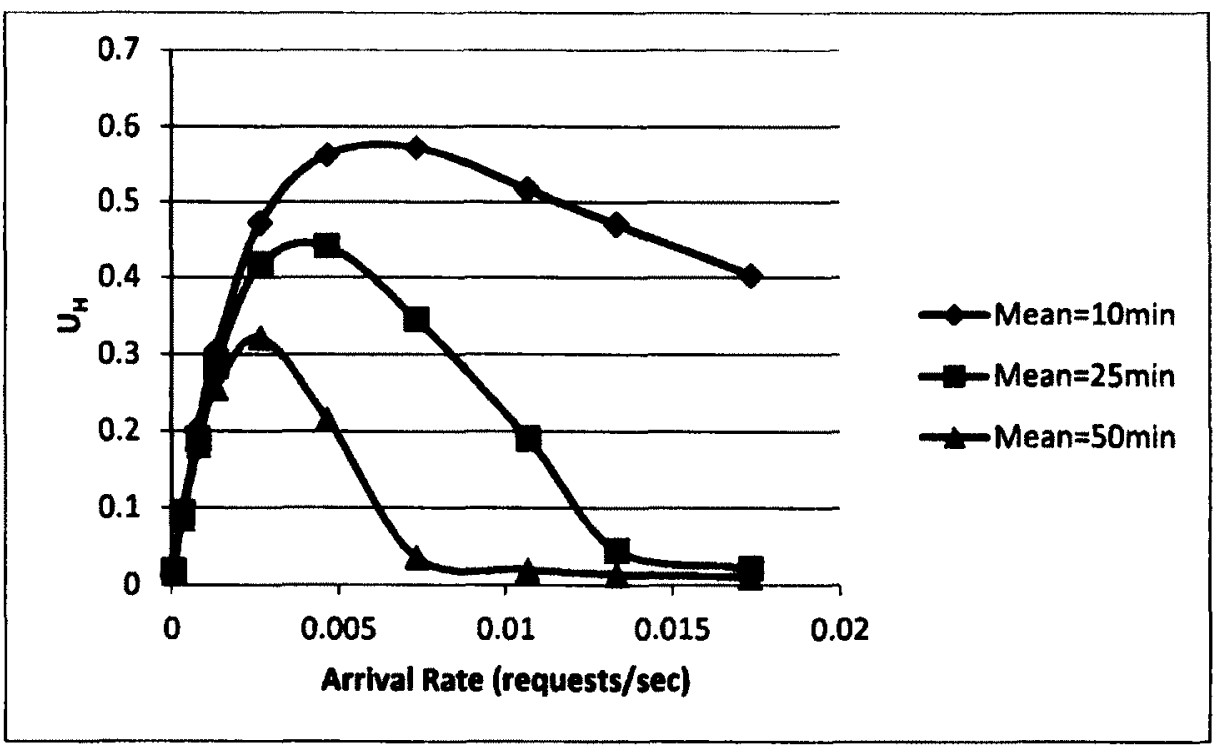

Figure 5-43: Impact of the Mean Emergency Service Time on the Average Resource Utilization due to High Priority ARs $\left(\mathrm{U}_{\mathrm{H}}\right)$

Figure 5-44 presents the $A_{T}$ values for the various mean emergency service times. The rate of accepted requests for both low and high priority AR requests follow a similar trend to $A_{T}$. At low arrival rates, the mean ES value does not impact $A_{T}$ as there is low contention on the resources. The rate of accepted requests increases until emergency 
requests begin to saturate the system at which point a very large number of AR requests are rejected. Saturating the system with the emergencies, captured in the figure can occur as a result of large emergency service times, or a large proportion of emergencies, or very high arrival rates.

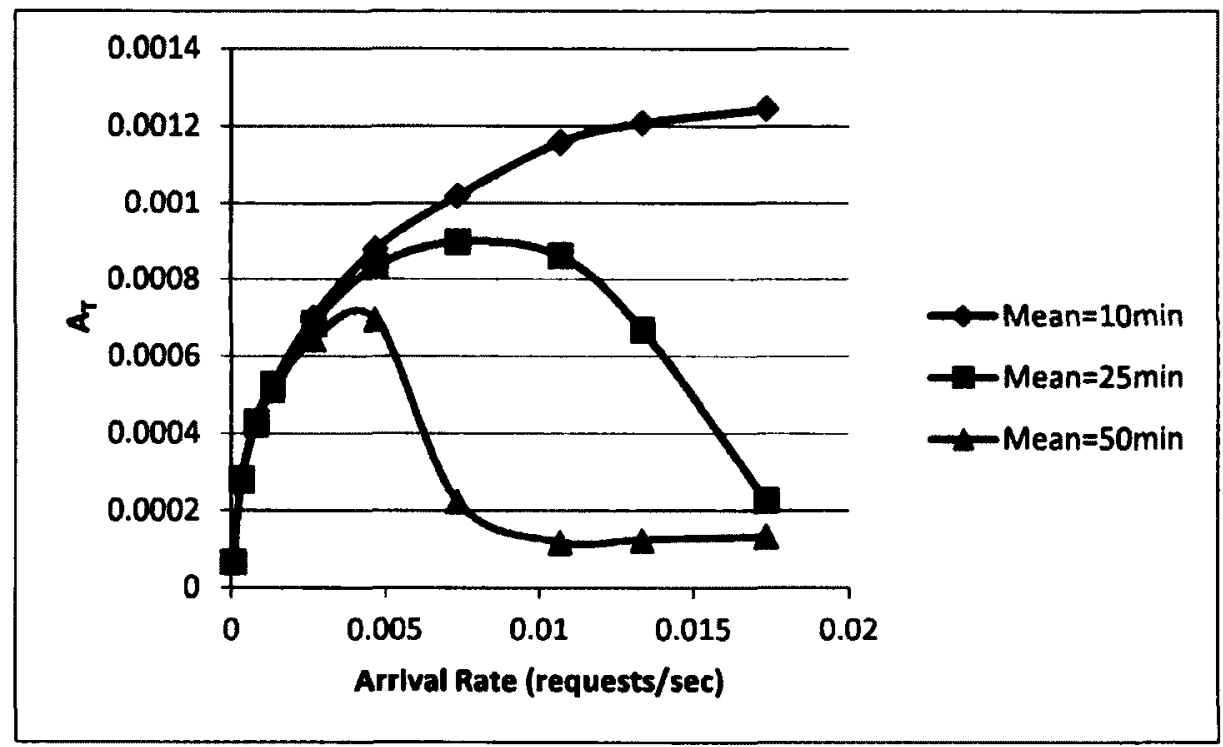

Figure 5-44: Impact of the Mean Emergency Service Time on the Overall Rate of Accepted Requests $\left(\mathbf{A}_{\mathbf{T}}\right)$

\subsubsection{Effect of the Proportion of Emergency Requests}

The impact of the proportion of Emergency requests (PE) on system performance is investigated here. In this set of experiments, PE is varied to subject the workload to various amounts of Emergency requests in the system. PE is set to $1 \%, 5 \%$, and $10 \%$. The arrival rates are the same as those used in other experiments with one resource. All other parameters are kept at their default values (see Table 5-1). Since the proportion of AR requests with high priority level is $10 \%$, the remaining proportion of requests have a low priority. 
Figure 5-45 shows the probability that an AR request is aborted (PA-E) for various proportions of Emergency requests. As expected, the results demonstrate that for a given arrival rate, as the proportion of Emergency requests in the system increase, there is a greater chance that an AR request is aborted. As the arrival rate increases, Emergency requests enter the system quicker. The faster Emergency requests arrive in the system, the number of emergencies that need to be serviced at a given time increases resulting in more AR requests (both low and high priority) being aborted, as seen in Figure 5-45.

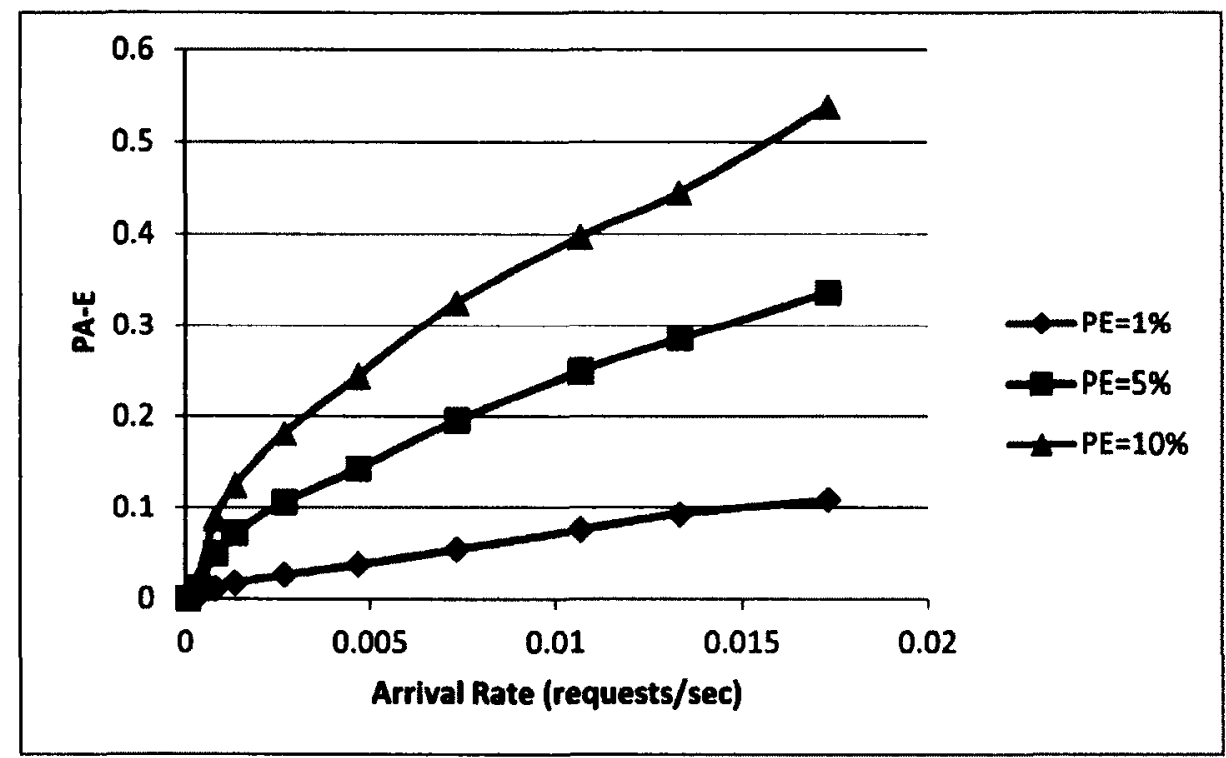

Figure 5-45: Impact of the Proportion of Emergencies (PE) on the Probabillty of Aborting ARs because of Emergencies (PA-E)

Figure 5-46 shows the average resource utilization due to Emergency requests $\left(U_{E}\right)$ for different proportions of Emergency requests. As expected, for a given arrival rate, as the proportion of emergencies increase, $U_{E}$ also increases, inherently decreasing the average resource utilization from high priority requests $\left(U_{H}\right)$ (see Figure 5-47) for the same reasons mentioned in Section 5.3.4.2. A similar trend is observed with an increase in the Emergency request's service time (see Figure 5-42). 
The $\mathrm{U}_{\mathrm{L}}$ graphs display a trend similar to the ones captured in Figure 5-33 and is included Appendix B, Figure B-6.

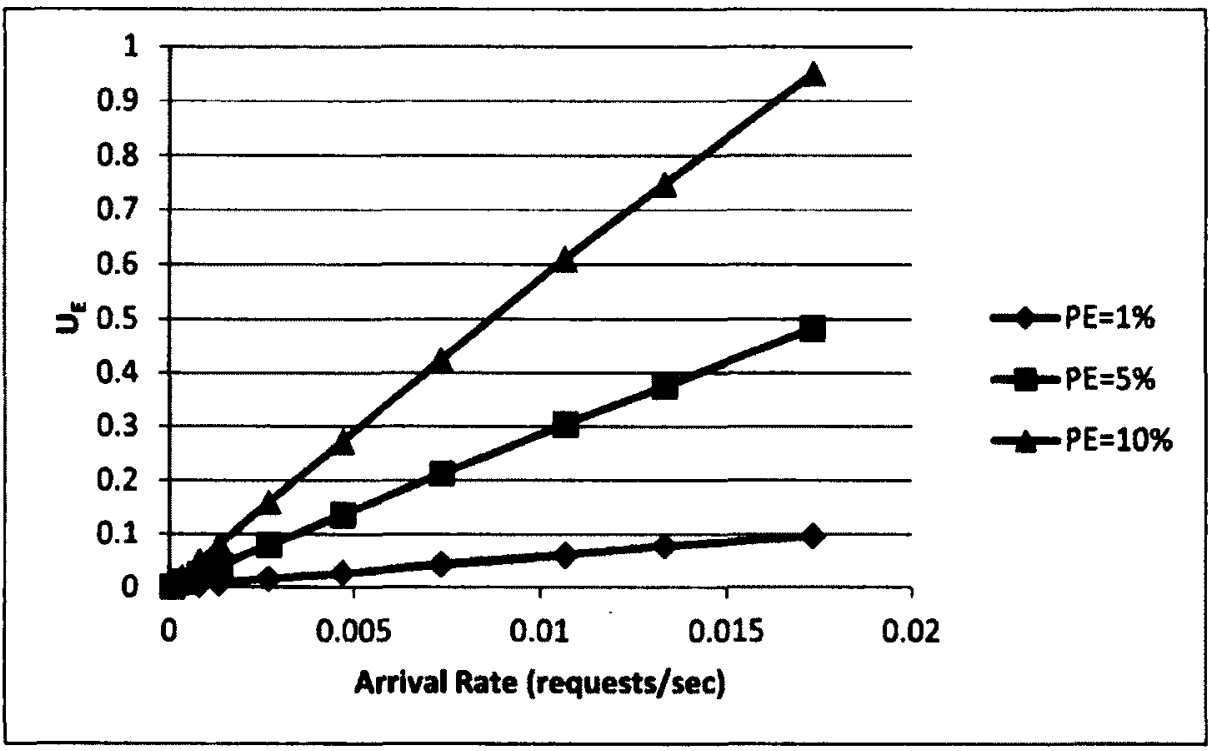

Figure 5-46: Impact of the Proportion of Emergencles (PE) on the Average Resource Utilization due to Emergencies $\left(U_{E}\right)$

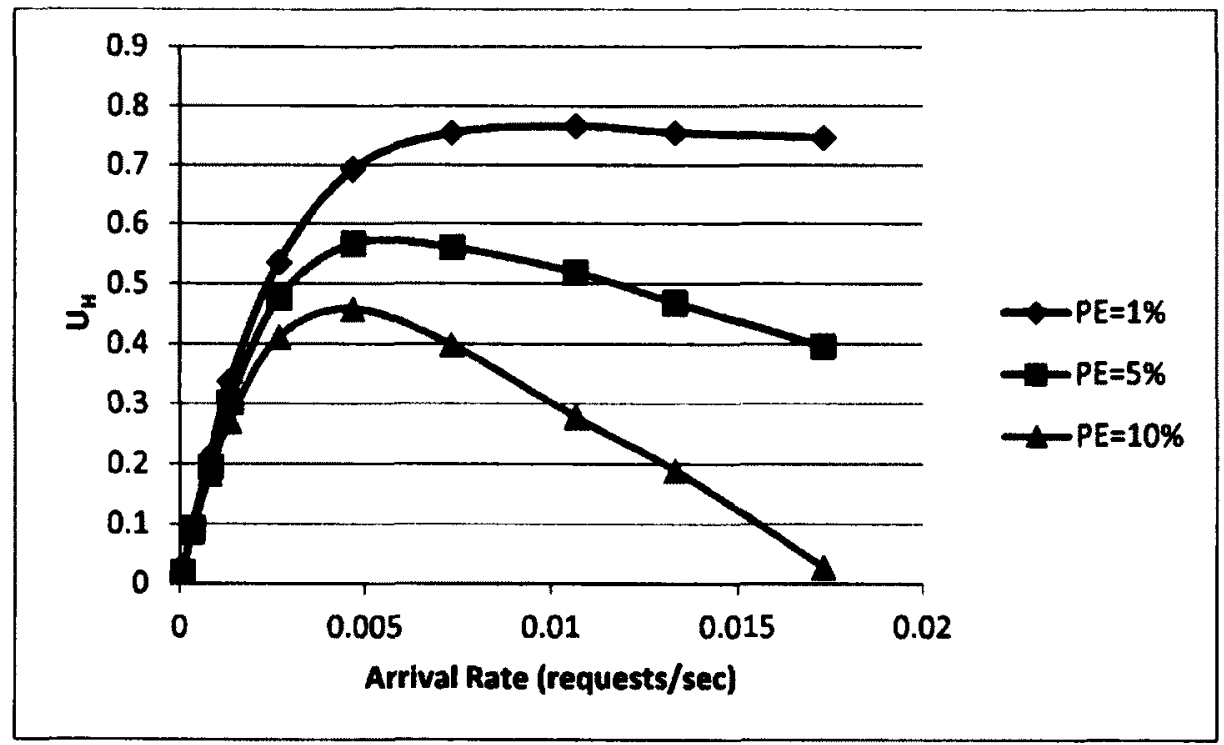

Figure 5-47: Impact of the Proportion of Emergencles (PE) on the Average Resource Utilization due to High Priority ARs $\left(U_{H}\right)$ 


\subsubsection{Effect of Padding Time}

This section studies the impact of adding padding to AR requests. Apart from Emergency requests, the workload in these simulations also consist of AR requests with multiple priorities; refer to Section 5.2.7 for the impact that padding has on the system for a workload without Emergency requests. As mentioned in Section 3.4, padding is used to artificially overestimate the AR request's service time by a specific duration referred to as padding time. Since the laxity of an AR request governs when its deadline is, padding time cannot be larger than the laxity. A percentage padding of $100 \%$ gives rise to a runtime of the request that is overestimated by the mean service time. The arrival rates are the same as those used in other experiments with one resource. All parameters are set to their default values (see Table 5-1). Since the percentage laxity is set to $200 \%$, the percentage padding cannot exceed this. The percentage padding values are set to $5 \%$, $50 \%$, and $200 \%$. As discussed in Section 3.4, the intention behind padding is that by adding additional idle time between the AR requests, the emergencies can use up the idle time so that the number of AR requests that are aborted can be reduced.

When an AR request completes, its padding time transforms into idle time on the resource. In such a situation two cases exist. In the first case, the next request begins immediately after the current request finishes or at its earliest start time, whichever comes first, thus ignoring the idle time. In the second case, the next request in the resource schedule begins execution after the idle time is finished. These two cases for when the next request begins execution are examined in the following section. For the experiments in this section, the next request ignores the idle time as in the first case. 
Figure 5-48 shows the impact that padding has on the probability of aborting an AR request because of an emergency (PA-E) for the different values of padding. It is shown that for low and medium arrival rates, as padding increases the chance of an AR request being aborted because of an emergency decreases. One explanation for this is that the Emergency request uses the time generated from padding which results in a lower number $\mathrm{AR}$ requests being aborted. Thus, as padding time increases, more time becomes available to service the emergency reducing the number of abortions. However, with the decrease in the number of requests being aborted comes a decrease in the overall average resource utilization $\left(U_{T}\right)$ as seen in Figure 5-49. As expected, at very high arrival rates, when emergencies saturate the system (see $U_{E}$ in 6.3B.5, Figure B-7), padding becomes irrelevant.

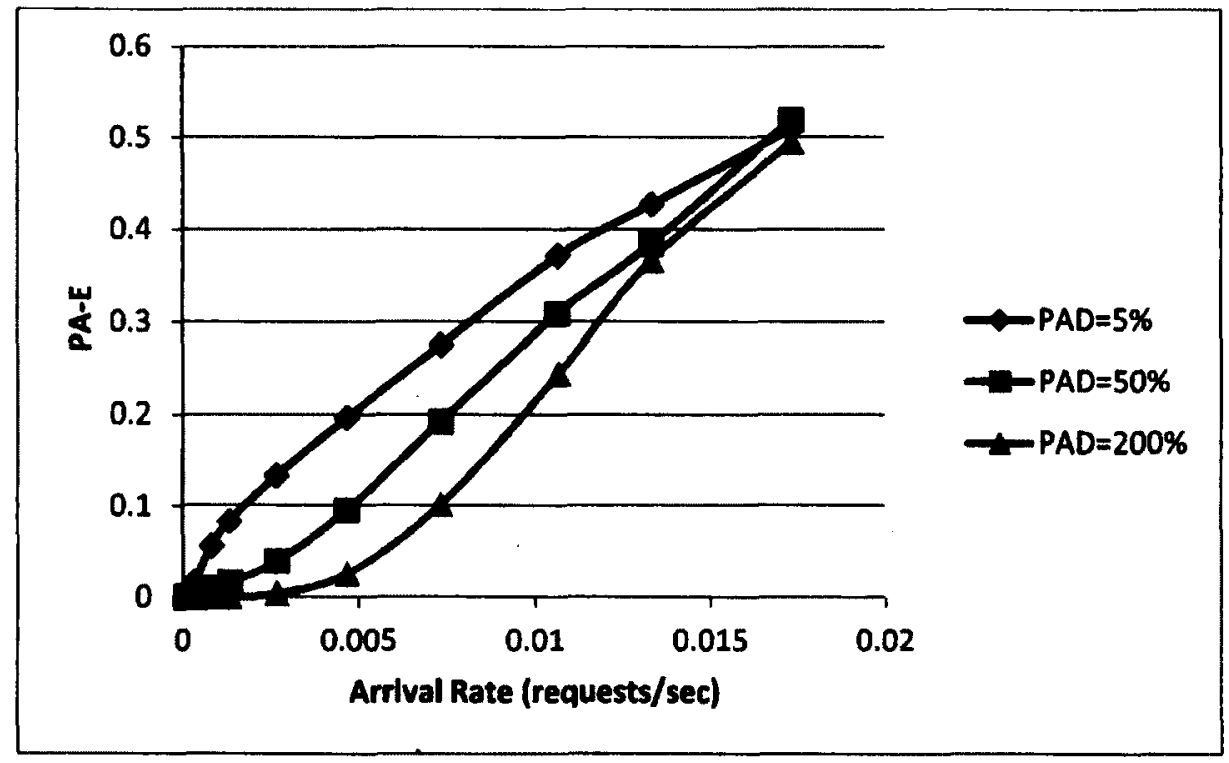

Figure 5-48: Impact of Padding on the Probability of Aborting ARs because of Emergencies (PA-E)

Since padding greatly affects the average resource utilization due to all requests $\left(U_{T}\right)$, an analysis of $U_{T}$ is provided. Similar to Section 5.2 .7 , as $U_{L}$ and $U_{H}$ do not provide any 
additional benefit when analysing padding, they will not be reported. Figure 5-49 shows the overall system utilization for different percentages of padding. As the arrival rate increases, the $U_{T}$ values also increase indicating that the Emergency requests are using up the idle time produced from padding. This is most notable with larger PAD values. It is observed that padding is more useful in the case of pre-emption when analysing $U_{T}$ for a given arrival rate. This can be observed by comparing $U_{T}$ in Figure 5-27 with the $U_{T}$ in Figure 5-49 for a given arrival rate and a given PAD value. As shown in Figure 5-49, for very low and high arrival rates, $U_{T}$ is insensitive to the different values of PAD. For intermediate arrival rates, $U_{T}$ is observed to decrease with an increase in padding. Thus, for a small to medium proportion of Emergency requests the overall system utilization seems to be adversely affected by an increase in padding time. This is captured in the figure.

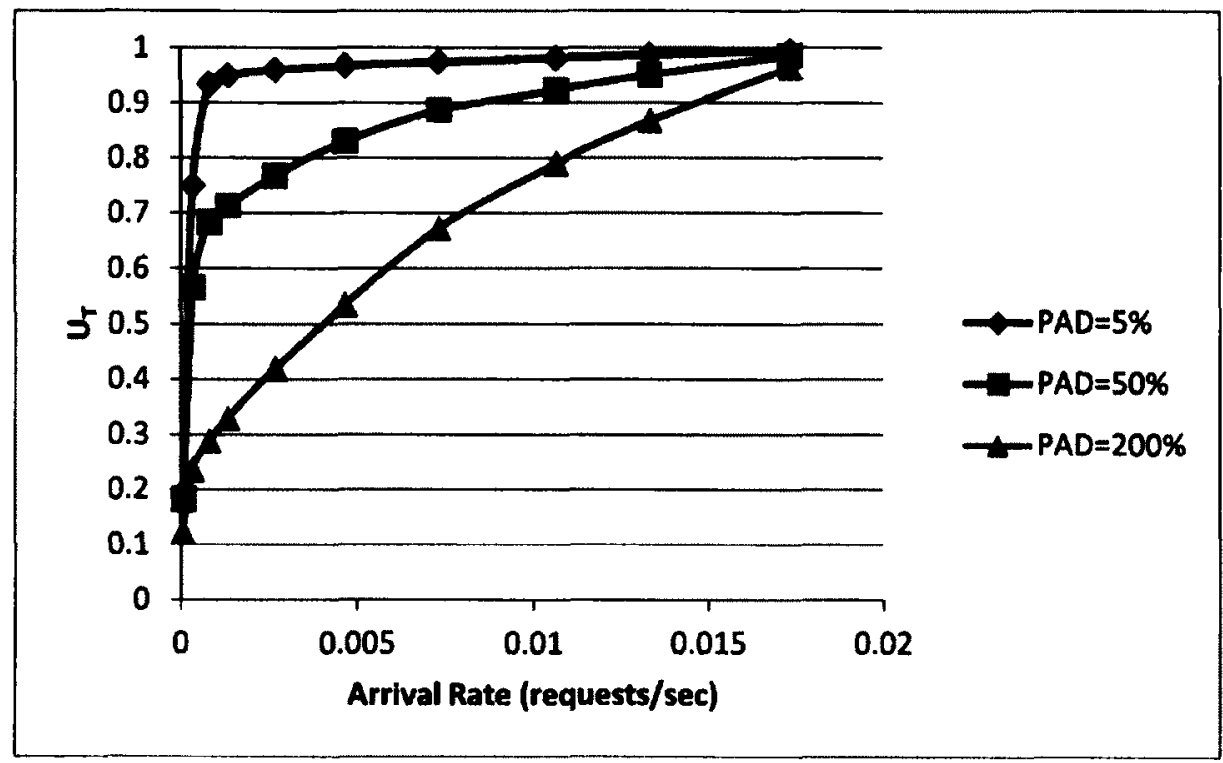

Figure 5-49: Impact of Padding on the Overall Average Resource Utilization $\left(U_{T}\right)$ 


\subsubsection{Effect of Next Request's Start Time}

This set of experiments studies the impact of the next AR request's start time on system performance. As mentioned in Section 5.3.6, two scenarios are investigated for when the next request in the resource schedule should begin execution at the time the current AR request finishes. In the first scenario, the idle time is either ignored where the next request commences immediately if possible, or at its earliest start time. In the second scenario, the next request waits until the idle time is finished to begin execution. The arrival rates are the same as those used in other experiments with one resource. All other parameters are set to their default values (see Table 5-1). The intention behind waiting until the idle time is finished is to provide additional time in case an emergency arrives.

Figure 5-50 shows the impact that the next request's start time has on the probability of aborting a request because of an emergency (PA-E). Two distinct levels of padding are experimented with for both scenarios; a low level of padding of 5\% and a high level of

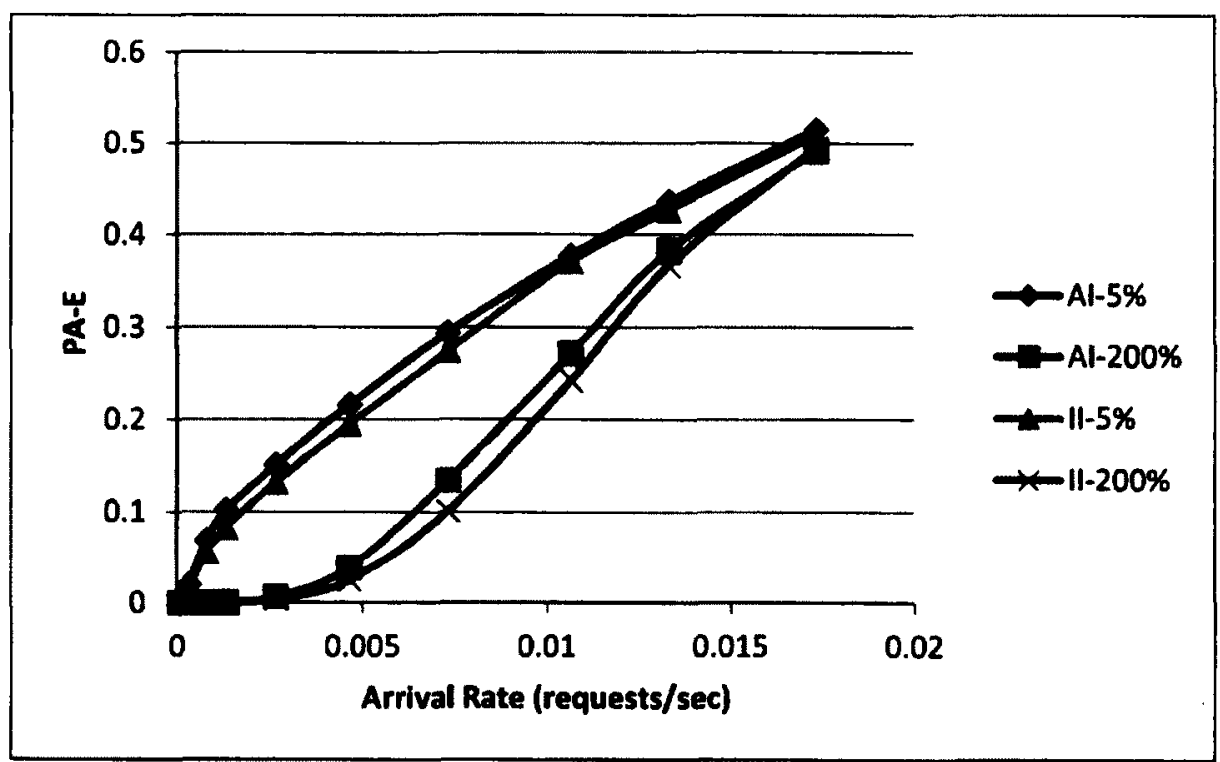

Figure 5-50: Impact of Next Request's Start Time on the Probability of Aborting ARs because of Emergencies (PA-E) 
padding of $200 \%$. The acronym AI "After Idle" refers to the scenario where the next request begins after the idle time, while II "Ignores Idle" refers to the scenario that ignores the idle time. For both levels of padding, the figure shows that there is a slightly lower chance of aborting an AR request because of an emergency when the idle time is ignored.

Figure 5-51 shows the impact that the next request's start time has on the overall utilization of the system $\left(\mathrm{U}_{\mathrm{T}}\right)$. For low levels of padding there is slight improvement on system utilization when the next request ignores the idle time. For high-levels of padding, both scenarios result in $U_{T}$ values that are statistically comparable to one another. For a given arrival rate, having the $U_{T}$ values close to each other for both scenarios indicates that there is no significant impact of whether or not the next request ignores the idle time on system performance. In addition, since large levels of padding result in large overestimations of $A R$ request service times, it is more difficult for the broker to schedule

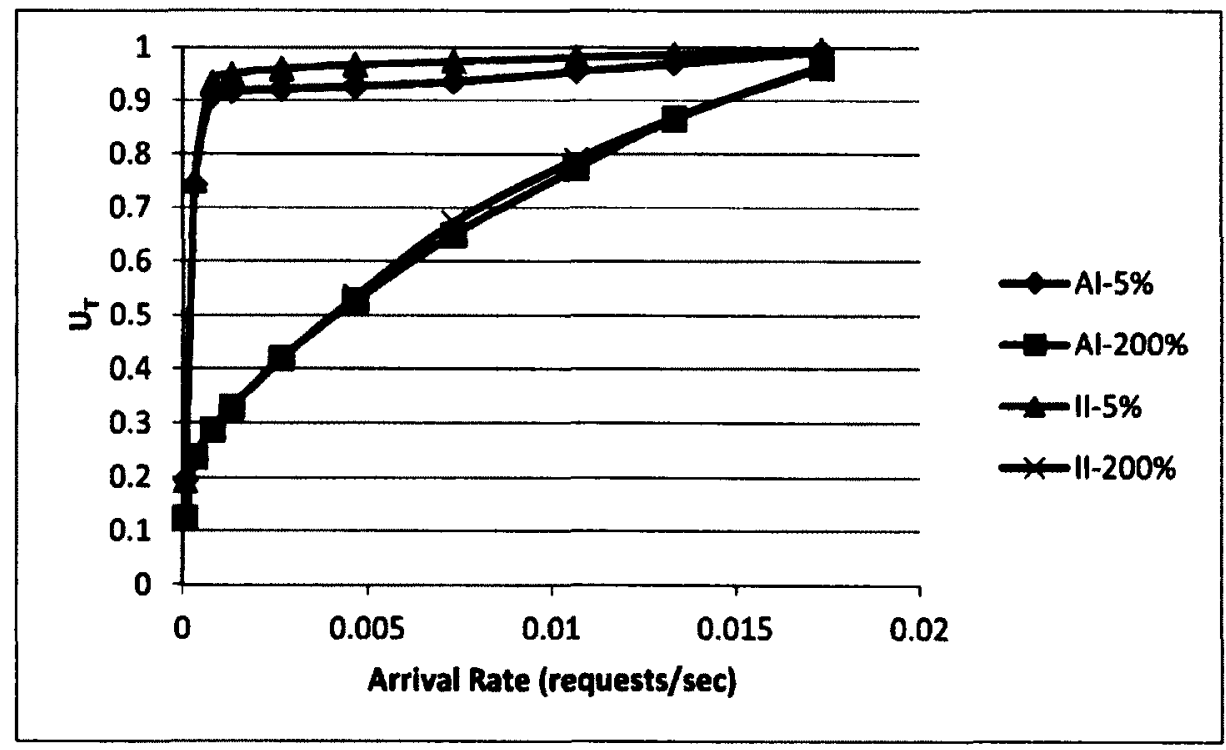

Figure 5-51: Impact of Next Request's Start Time on the Overall Average Resource Utilization $\left(U_{T}\right)$ 
the requests leading to an increase in resource contention. For these reasons, the next request's start time does not have a significant impact on system performance.

\subsection{Discussion}

A discussion of the key observations for the resource management techniques presented in this Chapter are outlined in this section. The allocation algorithms discussed in Section 5.2.3 did not seem to have any impact on the performance of the system. Through an analysis of the abortion policies used by the broker discussed in Section 5.2.1, it was determined that NR-A produced the worst system performance. The other abortion policies based on different characteristics of the system and/or workload, LS-A, GS-A, and NA-A were observed to have comparable system performances to one another for a given arrival rate and a given set of system and workload parameters. Since NA-A requires the lowest overhead, it seems to be the most attractive abortion policy. An evaluation of the pre-emption policies discussed in Section 5.3.1 reveals that the performance for all of the pre-emption policies are comparable to one another for a given arrival rate and a given set of system and workload parameters. Although NR-P comes with slightly higher PA-E values than the other pre-emption policies at medium and high arrival rates, it seems to be the most attractive pre-emption policy as the processing required for selecting a resource is greatly reduced. Padding is not recommended for use in the matchmaking strategies as it is observed to decrease system performance. Several of the system and workload parameters experimented with are observed to have a significant impact on the performance of the system. Improvements in system performance are observed when one or more of the following are increased: the number of resources in the resource pool, the variability factor of AR request service times, 
request laxity, or $T_{s}$. It is observed that knowing the emergency service time does not seem to have a significant impact on system performance. Similarly, the reallocation type also does not seem to have an impact on system performance. 


\section{Chapter 6 - Conclusions}

This chapter summarizes the research performed for this thesis. Section 6.1 provides a summary of the resource management techniques, as well as experimental set up for the simulations carried out in this thesis. Section 6.2 offers insights into the system behavior and performance under different workloads and system parameters. Finally, directions for future research are provided in Section 6.3.

\subsection{Summary}

Systems such as grids and clouds which are distributed in nature, consist of multiple resources that are used by clients. In these type of environments, resources are added and removed dynamically. To provide the most benefit to the resources it is necessary to consider the Quality of Service, QoS, requirements of different clients.

Typically grids and clouds are subjected to two types of requests known as On-Demand, OD, and Advance Reservation, AR, requests. OD requests are executed on a best effort basis, whereas AR requests have deadlines which are used to provide QoS guarantees that must be met. Another type of request, known as an Emergency request, has been developed for this thesis to incorporate systems that require emergency response. These requests include rare events that must be executed immediately. They have the highest priority level of all requests in the system and cannot be pre-empted.

As most existing works in the literature do not associate priorities with ARs, this thesis investigates techniques for dealing with ARs that are associated with multiple priority levels at runtime. As well, it also investigates techniques for dealing with an emergency response system. More specifically, it involves a grid or cloud system that handles a 
workload consisting of ARs with priorities and Emergency requests. For the reasons mentioned in Section 3.2.1, this research is concerned with three priority levels. Requests with priority level 0 represent low priority ARs, requests with priority level 1 signify high priority ARs, and requests with priority level 2 correspond to Emergency requests.

The broker that performs matchmaking uses the knowledge of the scheduling policy used at the resource. An Earliest Deadline First scheduling policy, described in Section 3.1.2.1, is used. When a request is schedulable on multiple resources without aborting or preempting any requests, the broker uses an allocation algorithm to select the resource for executing the request on. Two allocation algorithms that allocate the request using different criteria are presented. The allocation algorithms investigated are First Fit and Next Fit, described in Section 3.1.1. If the request is not schedulable on any resource and has the lowest priority level, it is rejected.

An incoming AR request having a priority level greater than 0 that is not accepted using the allocation algorithm, is handled by the abortion policies deployed at the broker to determine which lower priority request to abort. An abortion policy allows the broker to determine if the high priority AR request can be accommodated by aborting low priority AR requests. Provided the incoming request can be accommodated, the conflicting low priority request(s) is/are sent back to the broker for reallocation, and the high priority incoming request is scheduled. Otherwise the incoming request is rejected. Four abortion policies used for accommodating high priority AR requests using different system or workload characteristics are presented. They include: Greatest Sum of Service Times, GS-A, Largest Sum of Slack Times, LS-A, Next Abortable Resource, NA-A, and Next Resource, NR-A. Refer to Section 3.2.3 for a description of the abortion policies. 
Emergency requests are handled by the pre-emption policies deployed at the broker, and the pre-emptive scheduler. The pre-emption policies allow the broker to select a resource to execute the Emergency request. Four pre-emption policies using different system or workload characteristics are presented. They include: Greatest Service Time, GS-P, Largest Slack, LS-P, Shortest Time, ST-P, and Next Resource, NR-P. Refer to Section 3.3.3 for a description of the pre-emption policies.

In order to evaluate the performance of the resource management techniques, a simulator was developed. GridSim [BUY-02], a Java-based simulation toolkit was used to implement the various components including the source, the broker, the resources, and requests in the simulator. Various system and workload parameters were altered independently of one another in the simulation system to see their impact on the performance metrics. Extensive simulations were conducted in order to analyze the performance of the system and the resource management techniques. Various performance metrics were collected for all priority levels in the system. See Section 4.4 for the details regarding the performance metrics used.

\subsection{Insights into System Behaviour}

A number of key observations obtained from the simulation results are presented in this section.

\subsubsection{Impact of the Resource Management Techniques}

The insights gained for the resource management techniques employed by the broker for accommodating ARs with multiple priority levels, and emergencies are presented here. 
- The allocation algorithm (First Fit / Next Fit) used by the broker does not seem to have a significant impact on the performance of the system.

- NR-A gives rise to the abortion policy with the worst system performance. NR-A selects a resource based on the status of only one resource and it results in the highest $\mathrm{PB}_{\mathrm{H}}$, and $\mathrm{U}_{\mathrm{L}}$ values, and lowest $\mathrm{PA}$, and $\mathrm{U}_{\mathrm{H}}$ values of all abortion policies for a given arrival rate.

- Considering multiple resources for matchmaking seems to produce a significant improvement in performance. The performance of the other abortion polices, LSA, GS-A, and NA-A that do select a resource based on the status of more than one resource are observed to be statistically comparable to one another and are superior to the performance of NR-A for a given arrival rate. The most attractive among the abortion policies seems to be NA-A since it requires the lowest overhead while still producing a superior performance in comparison to NR-A.

- For a given arrival rate and a given set of workload and system parameters, all of the pre-emption policies outlined in this thesis produce results that are statistically comparable to one another. The most attractive pre-emption policy seems to be NR-P as it requires the lowest overhead while still producing results that are comparable to the other pre-emption policies.

\subsubsection{Impact of System and Workload Parameters on Performance}

Several workload and system parameters were experimented with throughout the simulations. NA-A which gave comparable performance to the other abortion policies (LS-A and GS-A) which achieved the highest performance is analysed further for various combinations of system and workload parameters. Similarly, since NR-P produced results 
that were comparable to the other pre-emption policies, it was chosen for analysing the impact of the system and workload parameters on performance. Insights gained into system behaviour and performance from the simulation results are presented.

- An increase in the variability factor of AR request service times results in an improvement in system performance. This is due to an increase in number of AR requests with smaller service times. Requests with smaller service times can be scheduled more effectively than requests with larger service times.

- An increase in the mean service time of $A R$ requests leads to reduced $A$, and $U_{L}$ values, and increased $P A, P B$, and $U_{M}$ values. This is a result of higher mean request service times increasing the amount of work the system is subject to. Since the system has a limited capacity, it tends to reject more requests as the mean service time increases.

- An increase in $T_{s}$ leads to requests staying longer at the resources. Low $T_{s}$ values (lower than 5 hours) causes requests to start sooner increasing contention at the resources. As a result, an increase in $T_{s}$ up to a value of 5 hours spread out the start time of the requests providing more flexibility for the scheduler to order the requests. This gives rise to an increase in $A, P A$, and $U$. $T_{s}$ values above 5 hours produce results that are statistically comparable to one another.

- As expected, an increase in laxity leads to an increase in the performance of the system. As laxity increases, the system has more flexibility in scheduling AR requests. Thus, an increase in laxity gives rise to a decrease in $\mathrm{PB}_{\mathrm{H}}$, and $\mathrm{U}_{\mathrm{L}}$, and an increase in $A_{H}, P A$, and $U_{H}$. 
- Padding was investigated for reasons mentioned in Section 3.4. An increase in padding leads to a decrease in system performance. As padding increases, the overestimation of the AR service time increases thus increasing contention on the resources. As a result, as padding increases there is a substantial decrease in A, and $U$. Thus, padding is not recommended for the use in the context of the resource management techniques considered in this thesis.

- An increase in the proportion of $\mathrm{AR}_{\mathrm{H}}$ requests has a significant impact of system performance. As $\mathrm{PAR}_{\mathrm{H}}$ increases, more $\mathrm{AR}_{\mathrm{H}}$ requests are submitted to the system increasing $A_{H}, P A, P B_{H}, A_{H}$, and $U_{H}$, and a decrease in $U_{L}$. Since, $A R_{L}$ requests cannot abort other requests, $\mathrm{PB}_{\mathrm{L}}$ is unaffected by $\mathrm{PAR}_{\mathrm{H}}$.

- Whether the emergency service time is known or not does not seem to have a significant impact on the performance of the system for the system and workload parameters that were experimented with. Indicated by the log files collected during simulation runs, this is a result of an extremely low number of requests that actually get reallocated when the emergency service time is known.

- Similarly, when the emergency service time is known and an AR request is preempted and reallocated, resuming or restarting the request does not seem to have a significant impact on the performance of the system for the experiments described in this thesis. Indicated by the log files collected during simulation runs, this is a result of a very small number of aborted requests actually being reallocated on a different resource. 


\subsection{Future Research}

This section presents a number of issues that warrant further investigation.

- Investigation of the matchmaking strategies for ARs with multiple priority levels when there is an error associated with the user estimated run times of the requests is a direction for future research.

- Investigation of the performance of the matchmaking strategies using different distributions of request run times is another area that could be considered for future research.

- Investigation of the performance of the system and matchmaking strategies with a broker that uses the Any-Schedulability criterion, described in Section 2.5.1, allowing the broker to perform the matchmaking for the requests without knowing the exact local scheduling policy at the resources forms an interesting direction for future research.

- The experimental results seen in this research are obtained using a simulation environment where the network delay is assumed to be negligibly small. An investigation into the matchmaking strategies using real workloads and system parameters such as real network delays is worthy of future research. 


\section{References}

[AHU1-09] R. Ahuja, G. Gabrani, A. De, "A Dynamic Priority Scheduler for Advance Reservation in Grid Computing", in the International Journal of Soft Computing, Vol. 4, No. 2, pp. 60-67, 2009.

[AHU2-09] R. Ahuja, A. De, G. Gabrani, "Negotiation Based Advance Reservation Priority Grid Scheduler with a Penal Cause for Execution Failures", in the 24th International Symposium on Computer and Information Sciences, pp. 522-530, Ankara, Turkey, September 2009.

[AKH-11] J. Akhani, S. Chuadhary, G. Somani, "Negotiation for Resource Allocation in IaaS Cloud", in the Proceedings of the Fourth Annual ACM Bangalore Conference, Article No. 15, New York, NY, USA, 2011.

[ALT-12] Altair Grid Technologies, Portable Batch System, http:// www.openpbs.org. Accessed September 2012.

[AMA-12] Amazon Web Services, Amazon Elastic Compute Cloud, http://aws.amazon.com/ec2/, Accessed September 2012.

[BAB-11] M. A. Babar, M.A.Chaucan, "A Tale of Migration to Cloud Computing for Sharing Experiences and Observations", in the Proceedings of the 2nd International Workshop on Software Engineering for Cloud Computing, pp. 50-56, Waikiki, Honolulu, USA, May 2011.

[BAI-05] C. Bailey Lee, Y. Schwartzman, J. Hardy, and A. Snavely, "Are User Runtime Estimates Inherently Inaccurate?", in Job Scheduling Strategies for Parallel Processing, Vol. 3277, pp. 253-263, Berlin, Germany, 2005. 
[BAN-05] J. Banks, J.S. Carson II, B.L. Nelson, D.M. Nicol, Discrete-Event System Simulation, Fourth Edition, New Jersey, Pearson Prentice Hall, 2005.

[BAR-12] C. Barnatt, Cloud Computing, www.explainingcomputers.com/cloud, Accessed September 2012.

[BER-02] V. Berstis, "Fundamentals of Grid Computing", in IBM Redbooks Paper, 2002. http://www.redbooks.ibm.com/redpapers/pdfs/redp3613.pdf, Accessed November 2012.

[BIR-09] G. Birkenheuer, A. Brinkman, H. Karl, "The Gain of Overbooking", in the Proceedings of the 14in International Workshop on Job Scheduling Strategies for Parallel Processing, pp. 80-100, Rome, Italy, May 2009.

[BUY-02] R. Buyya, M. Murshed, "GridSim: a toolkit for the modeling and simulation of distributed resource management and scheduling for Grid computing", in Concurrency and Computation: Practice and Experience, 14(13-15), pp. 1175-1220, December, 2002.

[BUY-09] R. Buyya, C. S. Yeo, S. Venugopal, J. Broberg, I. Brandic, “Cloud Computing and Emerging IT Platforms: Vision, Hype, and Reality for Delivering Computing as the 5th Utility", in Future Generation Computer Systems, Vol. 25, Issue 6, pp. 599-616, June, 2009.

[CAS-00] H. Casanova, F. Berman, G. Obertelli, R. Wolski, "The AppLeS Parameter Sweep Template: User-Level Middleware for the Grid", in the Proceedings of the ACM/IEEE Conference on Supercomputing, pp. 60-79, Washington, DC, USA, 2000.

[DAT-12] The DataGrid Project, http://eu-datagrid.web.cern.ch/eu-datagrid/, Accessed September 2012. 
[DEA-08] J. Dean, S. Ghemawat, "MapReduce: Simplified Data Processing on Large Clusters", in Communications of the $A C M$, Vol. 51, No. 1, pp. 107-113, New York, NY, USA, January 2008.

[DIS-08] A. Di Stefano, G. Morana, D. Zito, "Advanced Reservation in Grid Using PBS", in the Workshop on Enabling Technologies: Infrastructure for Collaborative Enterprises, pp. 216-221, June 2008.

[DOR-06] M. Dorigo, M. Birattari, T. Stutzle, “Ant Colony Optimization", in the IEEE Computational Intelligence Magazine, Vol. 1, No. 4, pp. 28-39, November 2006.

[ELM-08] E. Elmroth, J. Tordsson, "A Grid Resource Broker Supporting Advance Reservations and Benchmark-Based Resource Selection", in the Journal of Future Generation Computer Systems, Vol. 24, No. 6, pp. 585-593, June 2008.

[EXT-12] Extreme Science and Engineering Discovery Environments, Overview, www.xsede.org/overview, Accessed September 2012.

[FAR-07] U. Farooq, "A Framework for Quality of Service Aware Resource Management in Multi-Institutional Grids", Ph.D. dissertation, Department of Systems and Computer Engineering, Carleton University, Ontario, Canada, 2007.

[FAR-09] U. Farooq, S. Majumdar, E.W. Parsons, “Achieving Efficiency, Quality of Service and Robustness in Multi-Organizational Grids", in the Journal of Systems and Software, Vol. 82, pp. 23-38, January 2009. 
[FOS-99] I. Foster, C. Kesselman, C. Lee, B. Lindell, K. Nahrstedt, A. Roy, “A Distributed Resource Management Architecture that Supports Advance Reservations and CoAllocation", In the Proceedings of the International Workshop on Quality of Service, pp. 2736, London, UK, 1999.

[FOS-01] I. Foster, C. Kesselman, S. Tuecke, "The Anatomy of the Grid: Enabling Scalable Virtual Organizations", in the International Journal of Supercomputer Applications, Vol. 15, Issue 3, pp.200-222, Fall 2001.

[FOS-02] I. Foster, "What is the Grid? A Three Point Checklist", in GRID Today, July 2002, http://www.mcs.anl.gov/ itf/Articles/WhatIsTheGrid.pdf, Accessed September 2012.

[FOS-03] I. Foster , J. Vöckler , M. Wilde , Y. Zhao, "The Virtual Grids: a New Model and Architecture for Data Intensive Collaboration", in the Proceedings of the 15th International Conference on Scientific and Statistical Database Management, pp.11-24, Cambridge, Massachusetts, USA, July 2003.

[FOS-04] I. Foster, C. Kesselman, The Grid 2: Blueprint for a New Computing Infrastructure, Second Edition, San Francisco, CA: Morgan Kaufmann Publishers, 2004.

[FOS-08] I. Foster, Y. Zhao, I. Raicu, S. Lu, "Cloud Computing and Grid Computing 360Degree Compared", in Grid Computing Environments Workshop, pp. 1-10, November 2008.

[GAT-09] A.F. Gates, O. Natkovich, S. Chopra, P. Kamath, S.M. Narayanamurthy, C. Olston, B. Reed, S. Srinivasan, U. Srivastava, "Building a high-level Dataflow System on Top of Map-Reduce: the Pig Experience", in the Proceedings of the Very Large Database Endowment, Vol. 2, No. 2, pp. 1414-1425, August 2009. 
[G00-12] Google, Google Compute Engine, https://cloud.google.com/files/GoogleComputeEngine.pdf, Accessed September 2012.

[HAC-01] T.J. Hacker, B.D. Athey, "A Methodology for Account Management in Grid Computing Environments", in the Proceedings of the 2nd International Workshop on Grid Computing, pp. 133-144, 2001.

[HOA-10] D.B. Hoang, L. Chen, "Mobile Cloud for Assistive Healthcare (MoCAsH)", in the Proceedings of IEEE Asia-Pacific Services Computing Conference, pp. 325 - 332, Hangzhou, China, 2010.

[HOA-12] P.L. Hoang, "Soft Advance Reservation: A Resource Management Technique for Handling Errors in User Estimated Request Runtimes", M.A.Sc. Thesis, Department of Electrical and Computer Engineering, Carleton University, Ontario, Canada, 2012.

[IBM-12] IBM Platform Computing, "Platform's MultiCluster 8", http://info.platform.com/rs/platform/images/Datasheet_PlatformMultiCluster8.pdf, Accessed September 2012.

[JAC-03] B. Jacob, "Grid computing: What are the key components? Taking advantage of grid computing for application enablement", IBM, June 2003, www.cngrid.org/download/gridtechnology/gridbook/Gridcomputing.doc , Accessed June 2009.

[JAD-12] Y. Jadeja, K. Modi, "Cloud Computing - Concepts, Architecture and Challenges", in the Proceedings of the International Conference on Computing, Electronics and Electrical Technologies, pp. 877-880, Tamil Nadu, India, March 2012. 
[KIM-00] K. Kim, K. Nahrstedt, "A Resource Broker Model with Integrated Reservation Scheme", in the IEEE International Conference on Multimedia and Expo, Vol. 2, pp. 859862, New York, NY, USA, August 2000.

[KRA-02] K. Krauter, R. Buyya, M. Maheswaran, "A Taxonomy and Survey of Grid Resource Management Systems for Distributed Computing", in Software: Practice and Experience, Vol. 32, Issue 2, pp. 135-164, February 2002.

[KRE-10] M. Kretzschmar, S. Hanigk, "Security Management Interoperability Challenges for Collaborative Clouds", in the Proceedings of the 4th International DMTF Academic Alliance Workshop on Systems and Virtualization Management, pp. 43-49, Ontario, Canada, 2010.

[LAV-04] T. Lavian, J. Mambretti, D. Cutrell, H. Cohen, S. Merrill, R. Durairaj, P. Daspit, I. Monga, S. Naiksatam, S. Figueira, D. Gutierrez, D. Hoang, F. Travostino, "DWDM-RAM: A Data Intensive Grid Service Architecture Enabled by Dynamic Optical Networks", in the Proceedings of the 4th IEEE International Symposium on Cluster Computing and the Grid, pp.762-764, Chicago, Illinois, USA, April 2004.

[LI-11] K. Li, G. Xu, G. Zhao, Y. Dong, D. Wang, “Cloud Task Scheduling Based on Load Balancing Ant Colony Optimization", in the Proceedings of the 6th Annual ChinaGrid Conference, pp. 3-9, Dalain, Liaoning, China, 2011.

[LIT-61] J. D. C. Little, “A Proof for The Queuing Formula: $L=\lambda W ”$, Operations Research, Vol. 9, No. 3, pp. 383-387, 1961. 
[MAJ-09] S. Majumdar, "The Any Schedulability criterion for Providing QoS Guarantees through Advance Reservation Requests", in the Proceedings of the 9 th $I E E E / A C M$ International Symposium on Cluster Computing and the Grid (International Workshop on Cloud Computing), pp. 490-495, Shanghai, China, May, 2009.

[MAJ-12] S. Majumdar, M. Asif, J. Melendez, D. Lau, B. Nandy, M. Zaman, P. Srivastava, N. Goel, "Middleware for Sensor-Based Bridge Infrastructure Management", in the Proceedings of the 15th Communications and Networking Simulation Symposium, Article No. 8, San Diego, CA, USA, 2012.

[MAL-11] M. Malathi, "Cloud Computing Concepts", in the International Conference on Electronic Computers Technology, Vol. 6, pp. 236-239, Kanyakumari, India, April 2011.

[MEL1-11] P. Mell, T. Grance, "The NIST Definition of Cloud Computing", csrc.nist.gov/publications/nistpubs/800-145/PS800-145.pdf, Accessed September 2011.

[MEL2-11] J.O. Melendez, S. Majumdar, "Utilizing 'Opaque' Resources for Revenue Enhancement on Clouds and Grids", in the Proceedings of the 1lth IEEE/ACM International Symposium on Cluster, Cloud, and Grid Computing, pp. 576-584, Newport Beach, CA, USA, 2011.

[MER-12] Emergency, in Merriam-Webster's online Dictionary, http://www.merriamwebster.com/dictionary/emergency, Accessed September 2012.

[MIC-12] Microsoft Inc., Windows Azure, www.windowsazure.com/, Accessed September 2012. 
[MIN-01] R. Min, M. Maheswaran, "Scheduling Advance Reservation with Priorities in Grid Computing Systems", in the International Conference on Parallel and Distributed Computing Systems, pp. 172-176, Anaheim, CA, USA, 2001.

[MIN-02] R. Min, M. Maheswaran, "Scheduling Co-Reservations with Priorities in Grid Computing Systems", in the Proceedings of the 2nd IEEE/ACM International Symposium on Cluster Computing and the Grid, pp. 266-267, Berlin, Germany, 2002.

[MOS-12] I. Moschakis, H. Karatza, "Evaluation of Gang Scheduling Performance and Cost in a Cloud Computing System", in the Journal of Supercomputing, Vol. 59, No. 2, pp. $975-$ 992, 2012.

[OXY-12] Oxyzium InfoTech, Cloud Computing, http://oxyzium.blogspot.ca/2012/09/cloudcomputing.html, Accessed November 2012.

[PLA-02] B. Plale, P. Dinda, and G. von Laszewski, "Key Concepts and Services of a Grid Information Service", in the Proceedings of the 15th International Conference on Parallel and Distributed Computing Systems, pp. 437-442, Cambridge, MA, USA, November, 2002.

[PLA-12] Platform Computing, IBM Platform LSF, http://www.platform.com/Products, Accessed November 2012.

[PRO-12] R. Prodan, M. Sperk, S. Ostermann, "Evaluating High-Performance Computing on Google App Engine”, in IEEE Software, Vol. 29, Issue 2, pp. 52-58, April 2012.

[RAC-12] Rackspace, The Rackspace Open Cloud, http://www.rackspace.com/cloud/, Accessed September 2012. 
[RAN-11] S. Rani, R. Venkathesan, R. Ramalakshmi, "Resource Reservation in Grid Computing Environments: Design Issues", in the 3rd International Conference on Electronics Computer Technology, Vol. 4, pp. 66-70, Kanyakumari, India, April 2011.

[SHA-07] S. Shankar, D. J. DeWitt, "Data Driven Workflow Planning in Cluster Management Systems", in the Proceedings of the 16th International Symposium on High Performance Distributed Computing, pp. 127-136, New York, NY, USA, 2007.

[SMI-00] W. Smith, I. Foster, V. Taylor, "Scheduling with Advance Reservations", in the Proceedings of the IEEE/ACM 14thInternational Parallel and Distributed Processing Symposium, pp.127-132, Cancun, Mexico, May 2000.

[SOT-11] S. Sotiriadis, N. Bessis, N. Antonopoulos, "Towards Inter-cloud Schedulers: A Survey of Meta-Scheduling Approaches", in the Proceedings of the International Conference on P2P, Grid, Cloud and Internet Computing, pp. 59-66, Barcelona, Spain, October 2011.

[SUL-04] A. Sulistio, R. Buyya, "A Grid Simulation Infrastructure Supporting Advance Reservation", in the Proceedings of the 16inInternational Conference on Parallel and Distributed Computing and System, pp. 1-7, Boston, MA, USA, November 2004.

[VAQ-09] L. M. Vaquero, L. Rodero-Merino, J. Caceres, M. Lindner, "A Break in the Clouds: Towards a Cloud Definition", in the ACM SIGCOMM Computer Communication Review, Vol. 39, Issue 1, pp. 50-55, New York, NY, USA, January 2009.

[WAN-10] Y. Wang, B. Jin, "The Application of SaaS Model in Network Education-Take Google Apps for Example", in the 2nd International Conference on Education Technology and Computer, Vol. 4, pp. 191-194, Shanghai, China, June 2010. 
[WOL-99] R. Wolski, N. Spring, J. Hayes, "The Network Weather Service: A Distributed Resource Performance Forecasting Service for Metacomputing", in the Journal of Future Generation Computing Systems, Vol. 15, No. 5, pp. 757-768, October 1999.

[YUA-11] Y. Yuan, W. Liu, "Efficient Resource Management for Cloud Computing", in the Proceedings of the International Conference on System Science, Engineering Design and Manufacturing Information, Vol. 2, pp. 233-236, Guiyang, China, October 2011.

[ZHA-12] Z. Zhang, L. Cherkasova, A. Verma, B.T. Loo, "Meeting Service Level Objectives of Pig Programs", in the Proceedings of the 2nd International Workshop on Cloud Computing Platforms, No. 8, pp. 1-6, Bern, Switzerland, April 2012. 


\section{Appendix A}

\section{A.1 Impact of the Abortion Policy}

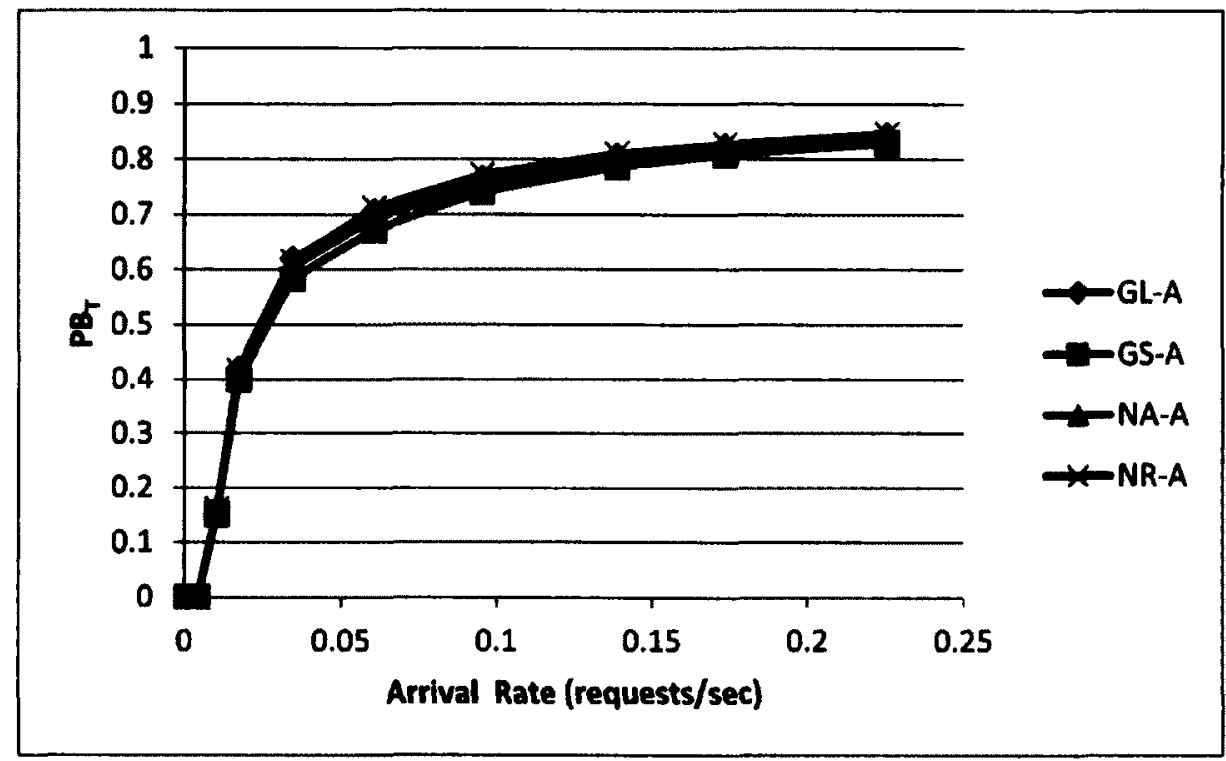

Figure A-1: Impact of the Abortion Policies on the Overall Probability of Blocking (PB $)$

\section{A.2 Impact of the AR Service Time Variability Factor}

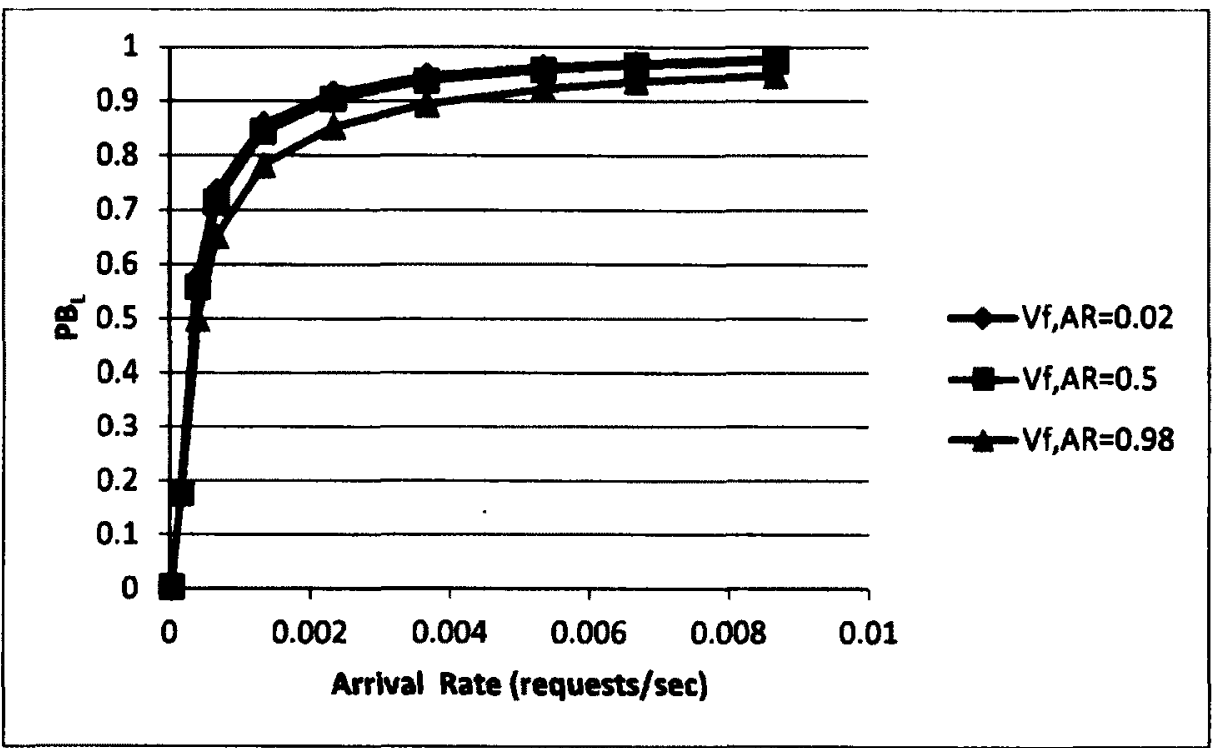

Figure A-2: Impact of Variability Factor for ARs $\left(V_{f, A R}\right)$ on the Probability of Blocking Low Priority ARs $\left(\mathbf{P B}_{\mathrm{L}}\right)$ 


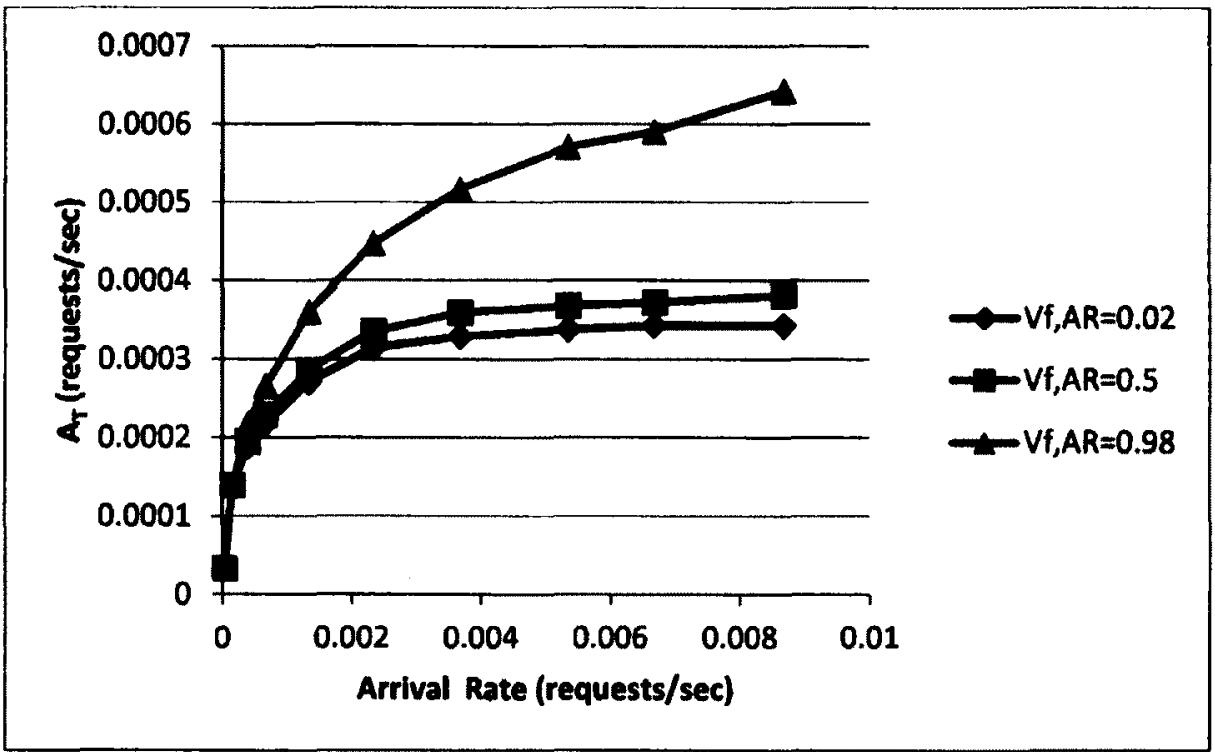

Figure A-3: Impact of Variability Factor for ARs $\left(V_{\mathrm{I}, \mathrm{AR}}\right)$ on the Overall Rate of Accepted Requests $\left(A_{T}\right)$

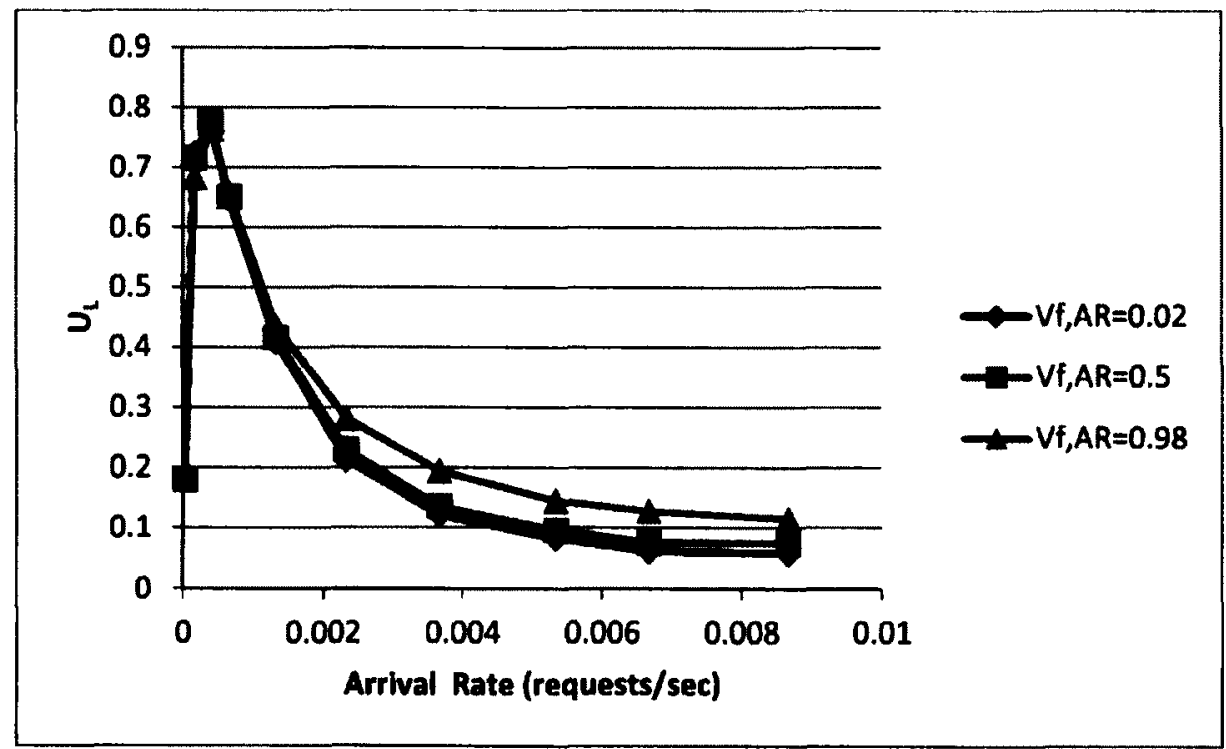

Figure A-4: Impact of Variability Factor for ARs $\left(V_{f, A R}\right)$ on the Average Resource Utillzation due to Low Priority ARs $\left(U_{L}\right)$ 


\section{A.3 Impact of Mean AR Service Time}

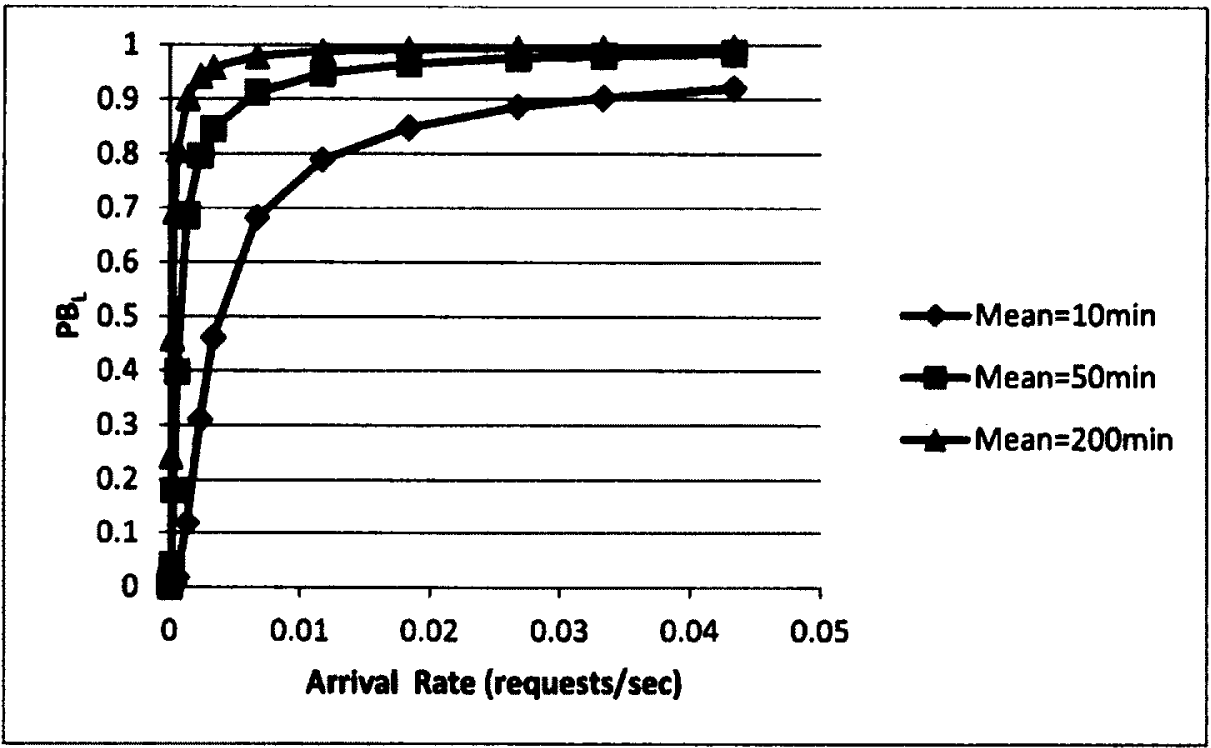

Figure A-5: Impact of the Mean AR Service Time on the Probability of Blocking Low Priority ARs $\left(\mathbf{P B}_{\mathbf{L}}\right)$

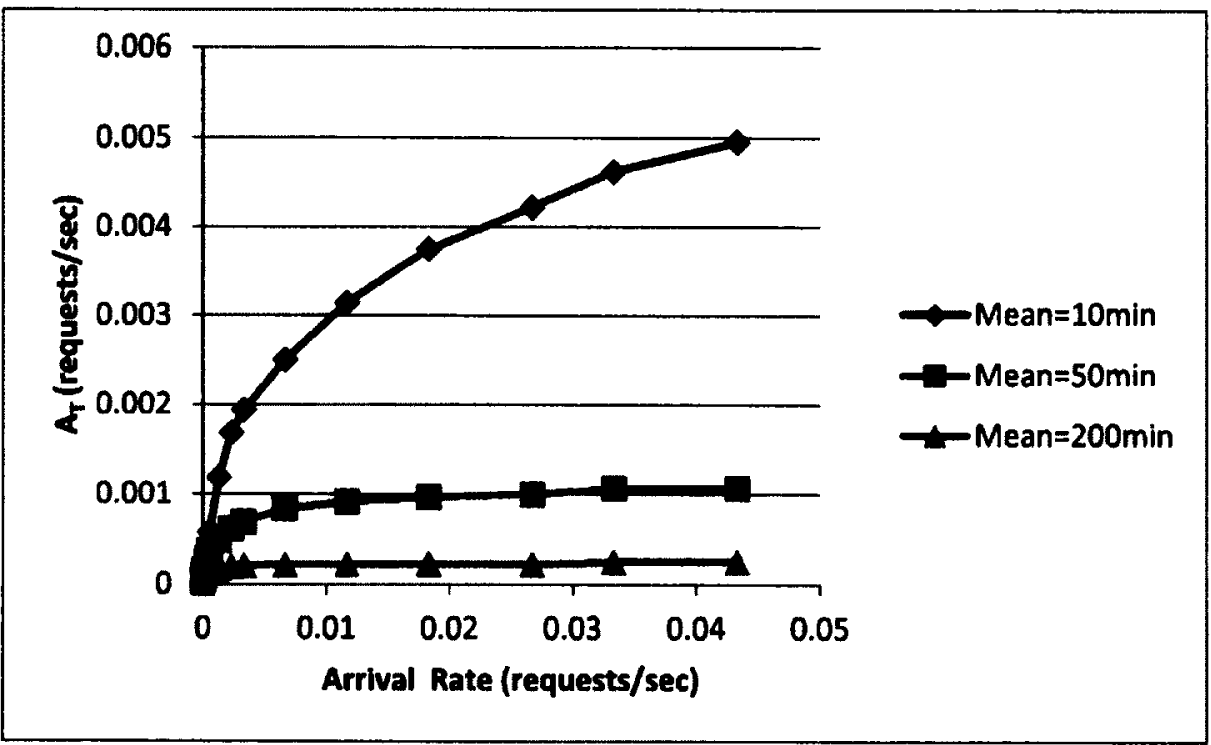

Figure A-6: Impact of the Mean AR Service Time on the Overall Rate of Accepted Requests $\left(A_{r}\right)$ 


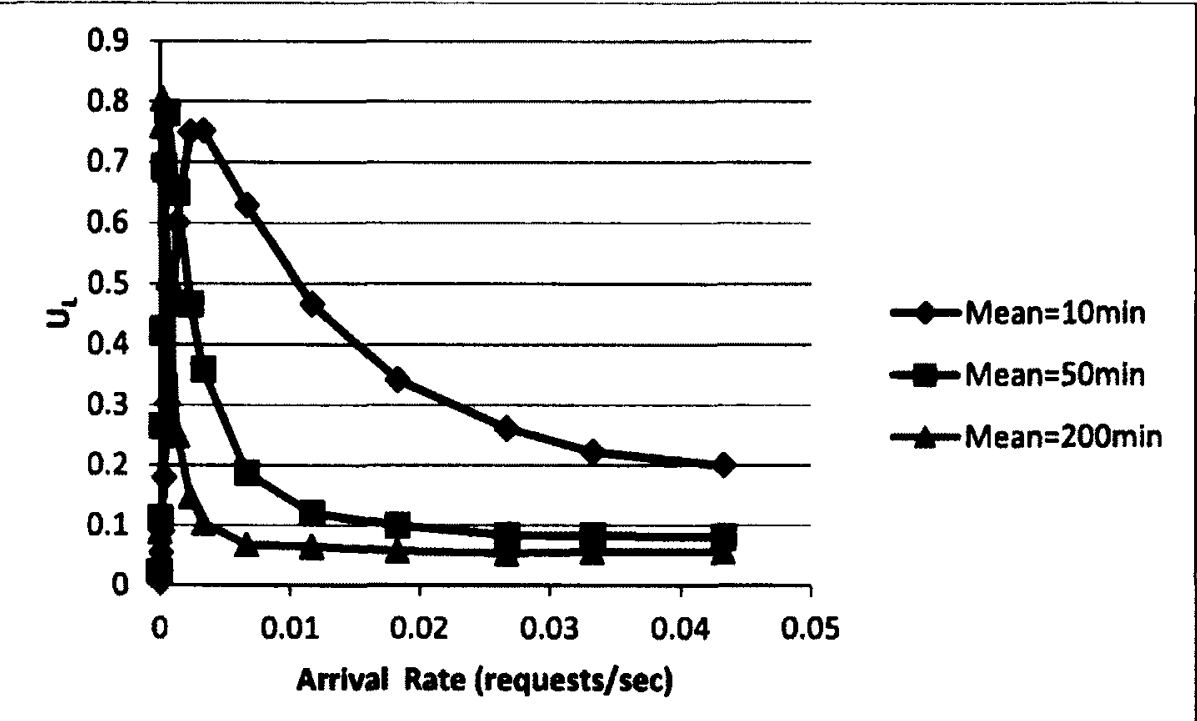

Figure A-7: Impact of the Mean AR Service Time on the Average Resource Utilization due to Low Priority ARs $\left(\mathbf{U}_{\mathrm{L}}\right)$

\section{A.4 Impact of Earliest Start Time}

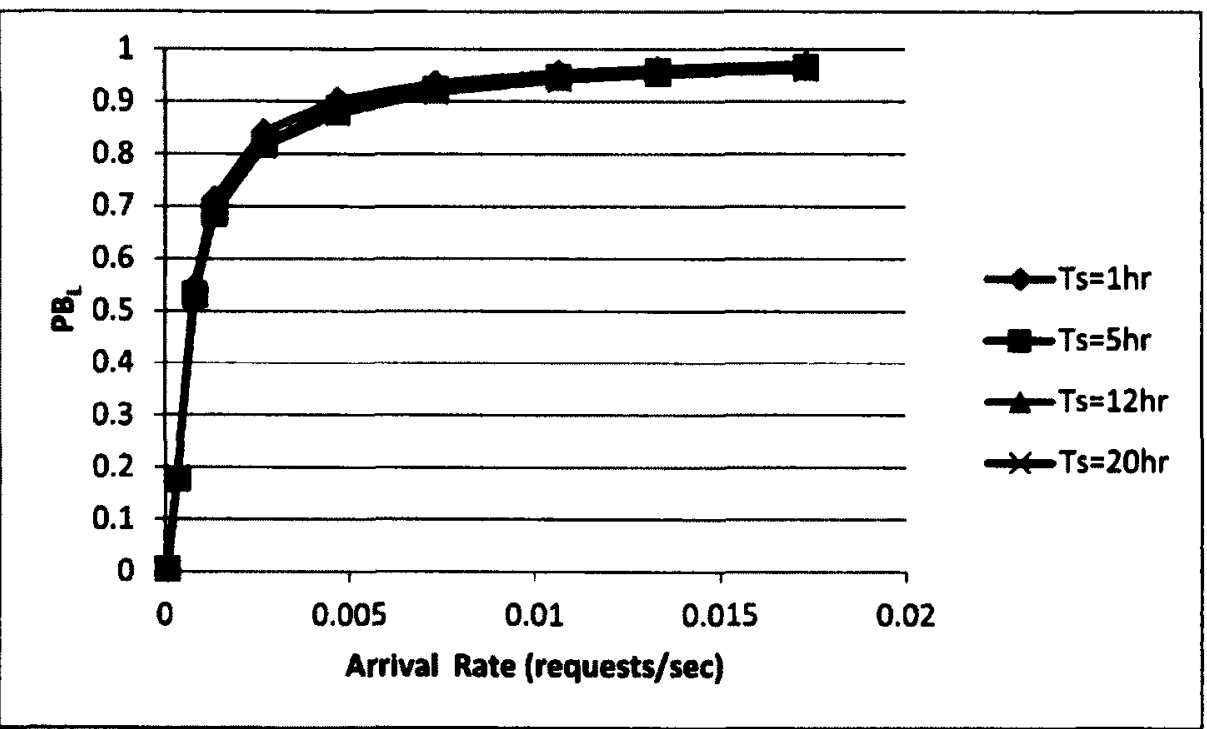

Figure A-8: Impact of T, on the Probability of Blocking Low Priority ARs $\left(\mathrm{PB}_{\mathrm{L}}\right)$ 


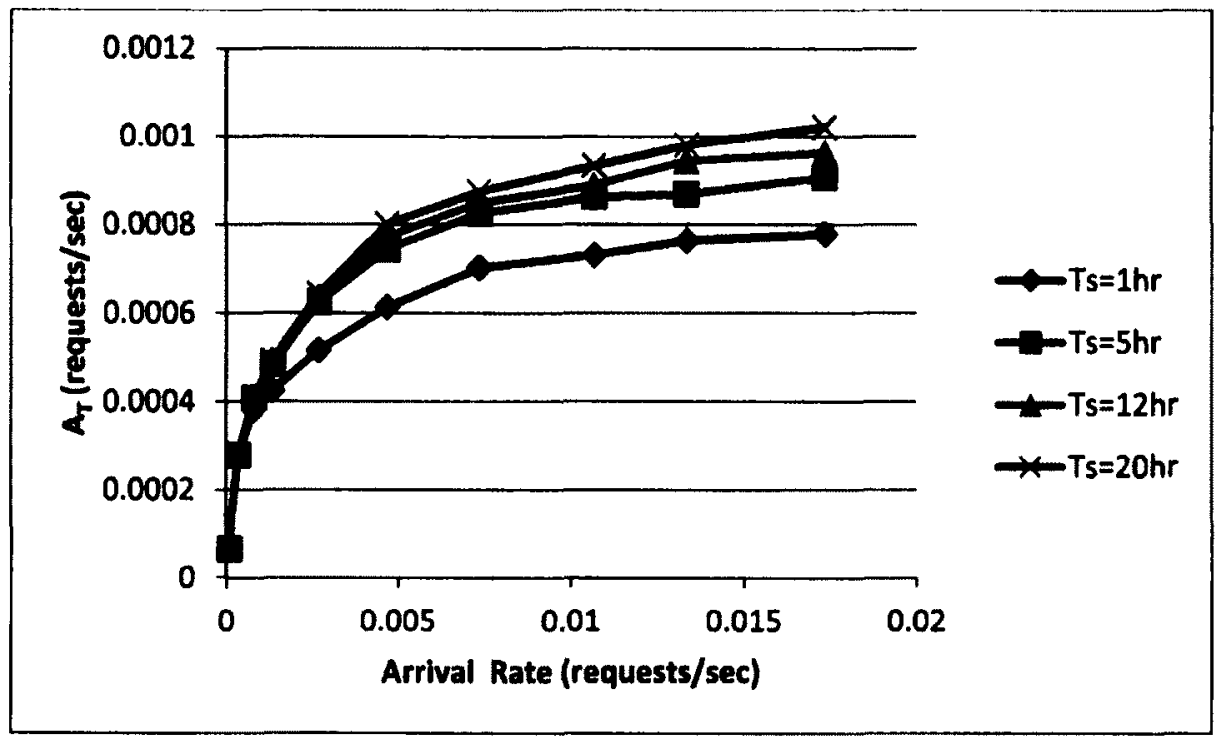

Figure A-9: Impact of $T$, on the Overall Rate of Accepted Requests $\left(A_{T}\right)$

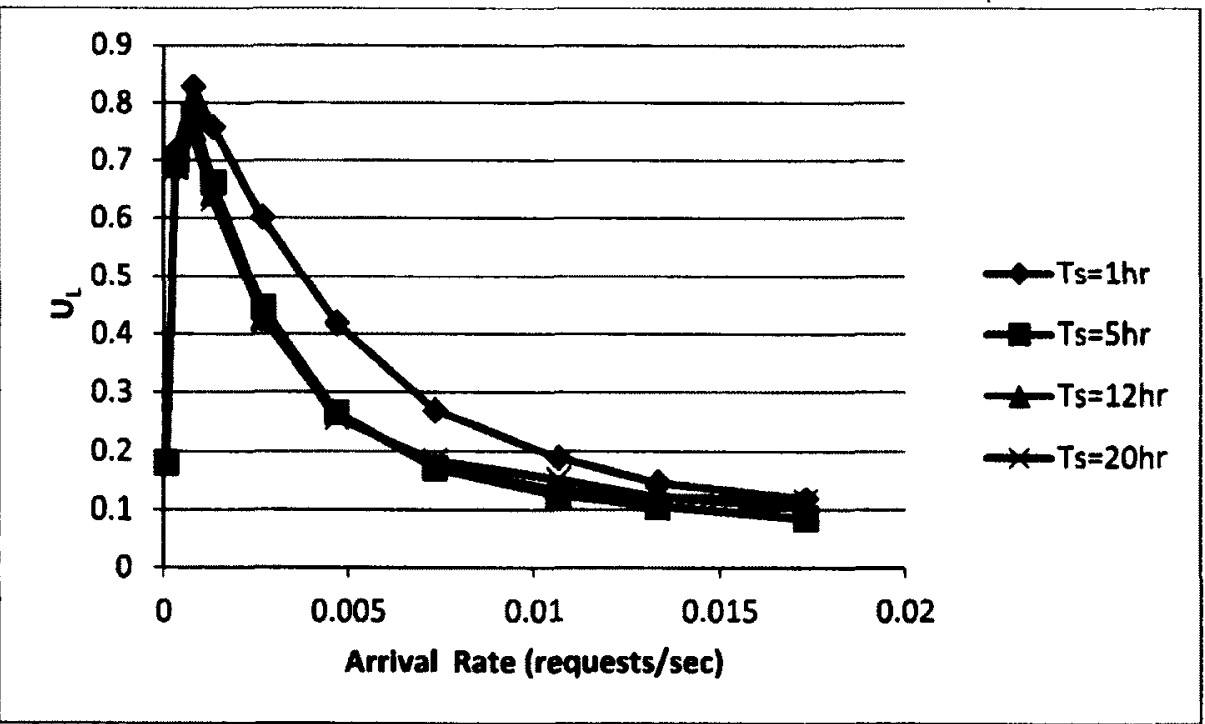

Figure A-10: Impact of $T_{3}$ on the Average Resource Utilization due to Low Priority ARs $\left(U_{L}\right)$ 


\section{A.5 Impact of Laxity}

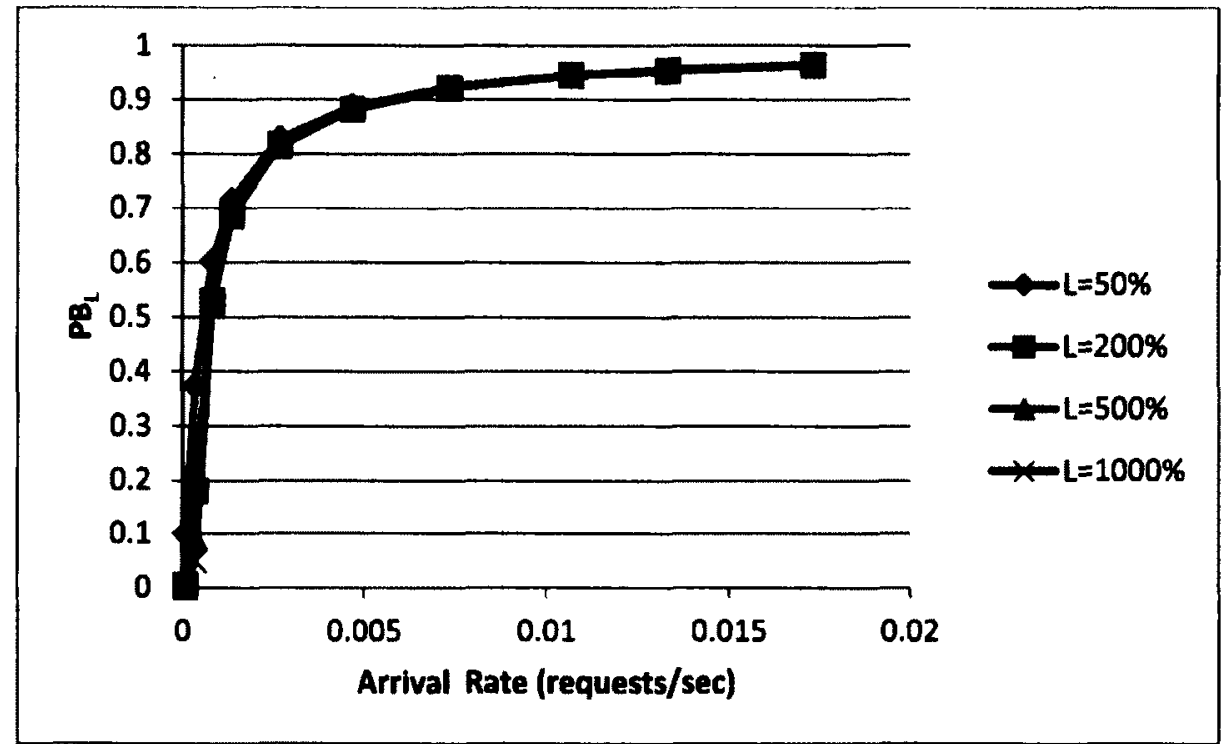

Figure A-11: Impact of Laxity (L) on the Probability of Blocking Low Priority ARs (PB $\left(\mathrm{B}_{\mathrm{L}}\right)$

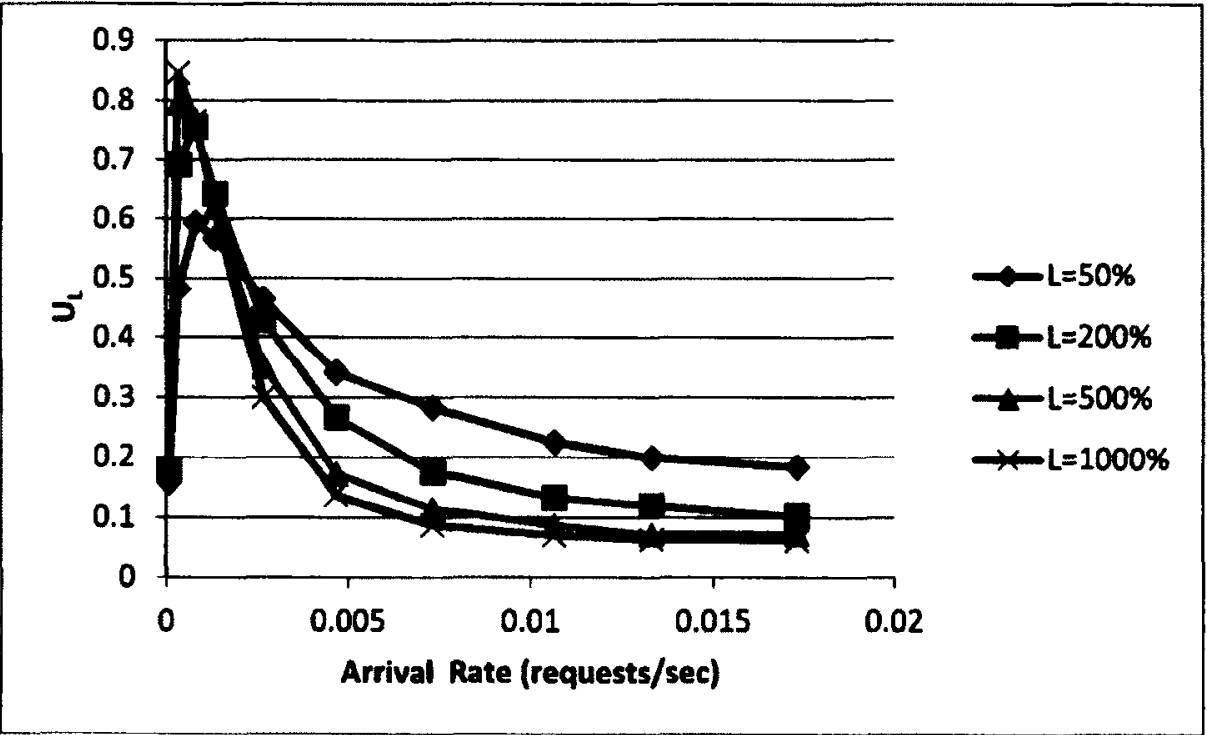

Figure A-12: Impact of Laxity (L) on the Average Resource Utilization due to Low Priority ARs $\left(U_{L}\right)$ 


\section{A.6 Impact of Padding Time}

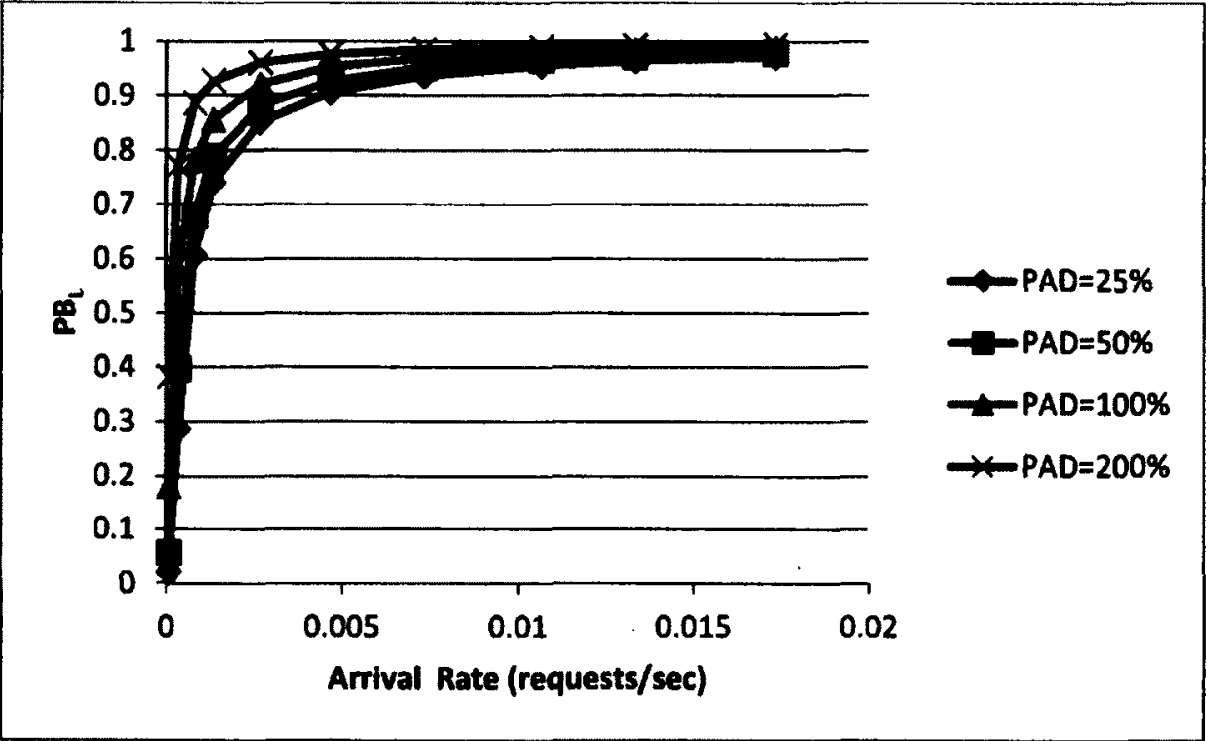

Figure A-13: Impact of Padding on the Probability of Blocking Low Priority ARs (PB ( $_{\mathrm{L}}$

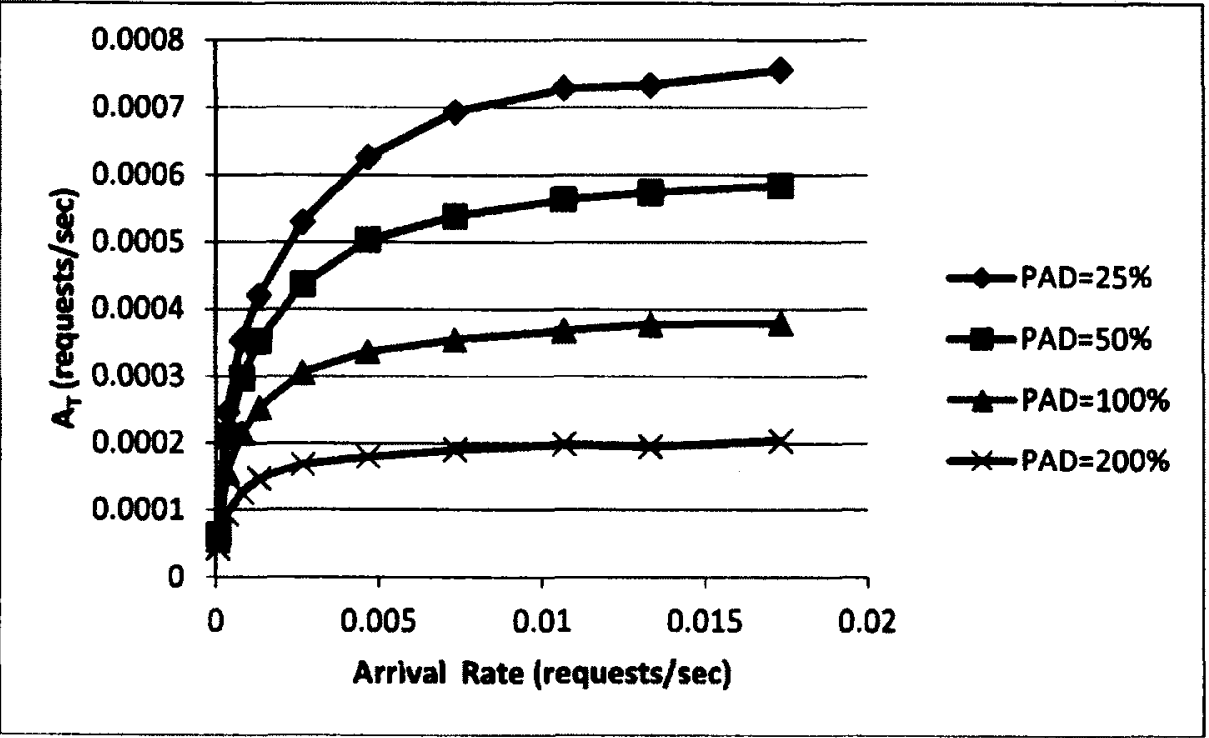

Figure A-14: Impact of Padding on the Overall Rate of Accepted Requests (AT) 


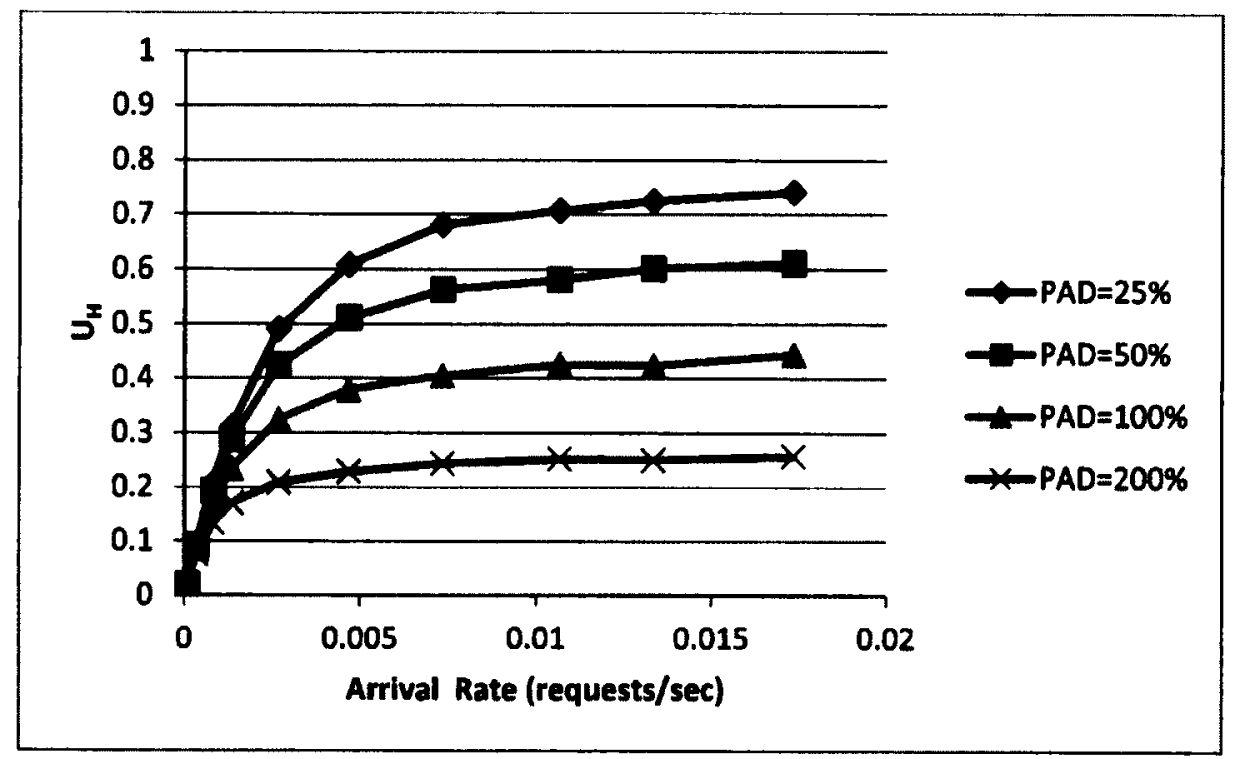

Figure A-15: Impact of Padding on the Average Resource Utilization of High Priority ARs $\left(\mathrm{U}_{H}\right)$

\section{A.7 Impact of the Proportion of High Priority AR Requests}

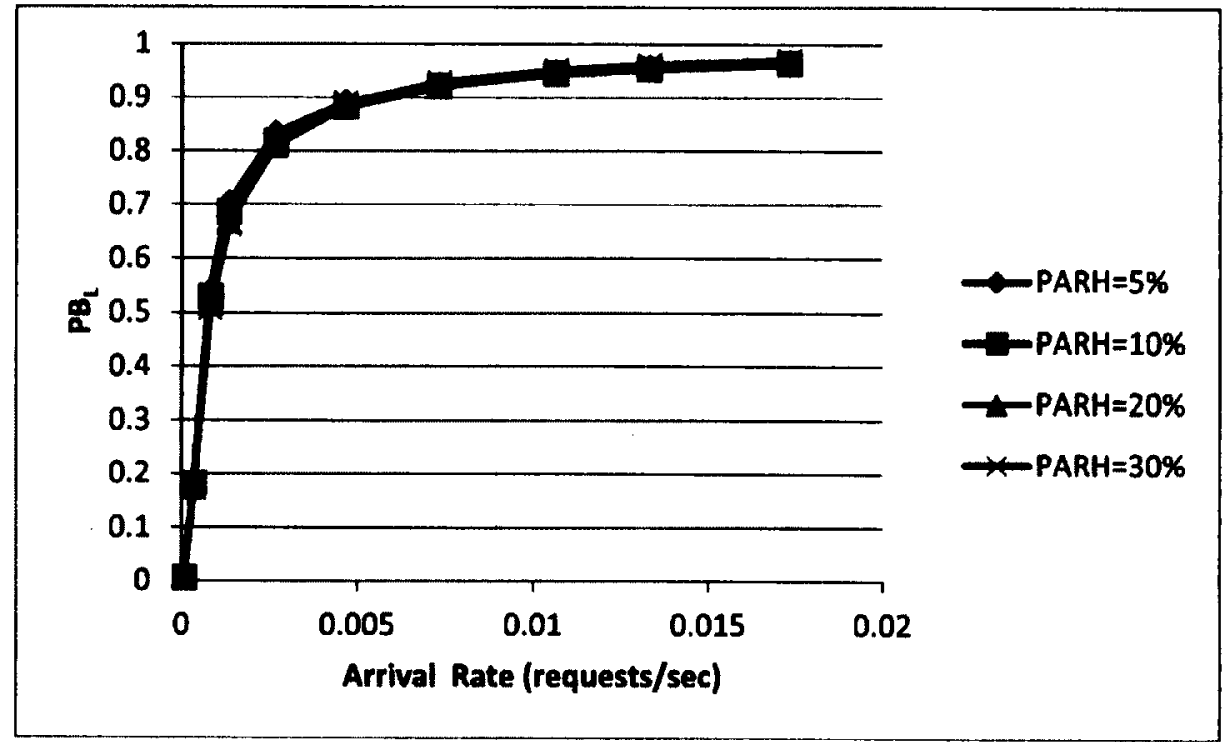

Figure A-16: Impact of the Proportion of High Priority ARs $\left(\right.$ PAR $\left._{H}\right)$ on the Probability of Blocking Low Priority ARs $\left(\mathbf{P B}_{\mathrm{L}}\right)$ 


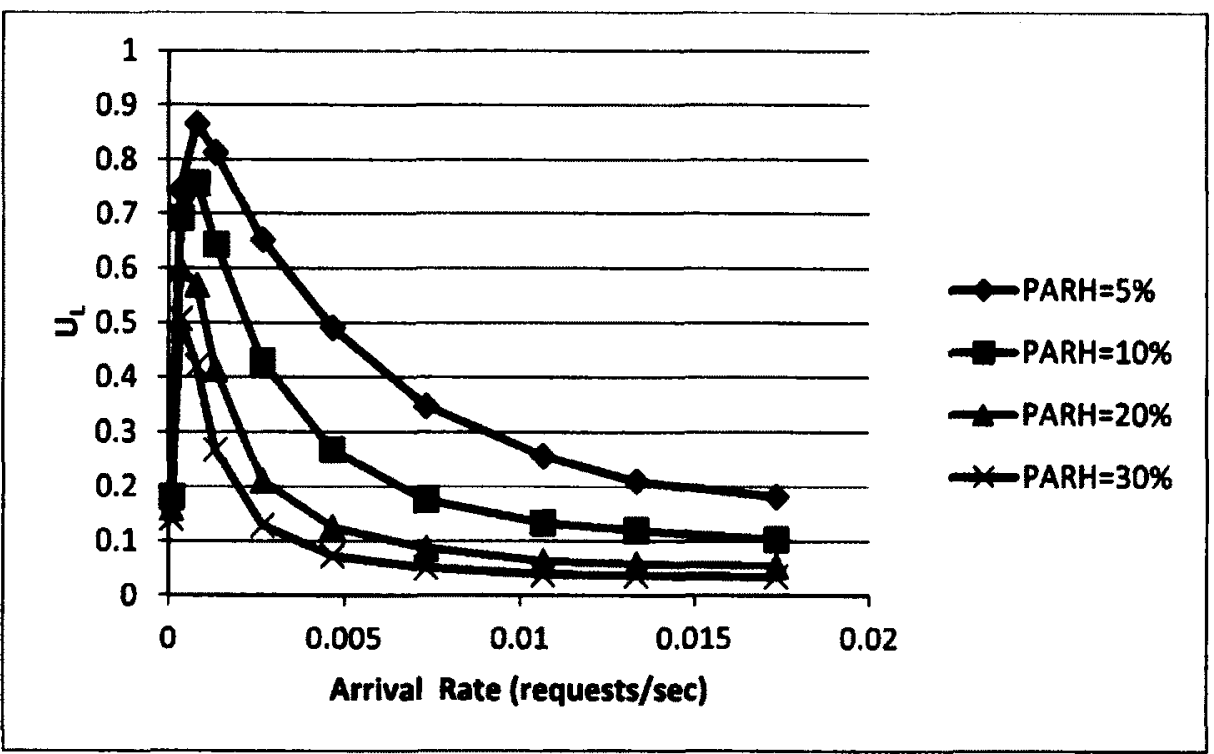

Figure A-17: Impact of the Proportion of High Priority ARs $\left(\mathbf{P A R}_{H}\right)$ on the Average Resource Utilization due to Low Priority ARs $\left(\mathrm{U}_{\mathrm{L}}\right)$ 


\section{Appendix B}

\section{B.1 Impact of the Pre-emption Policy}

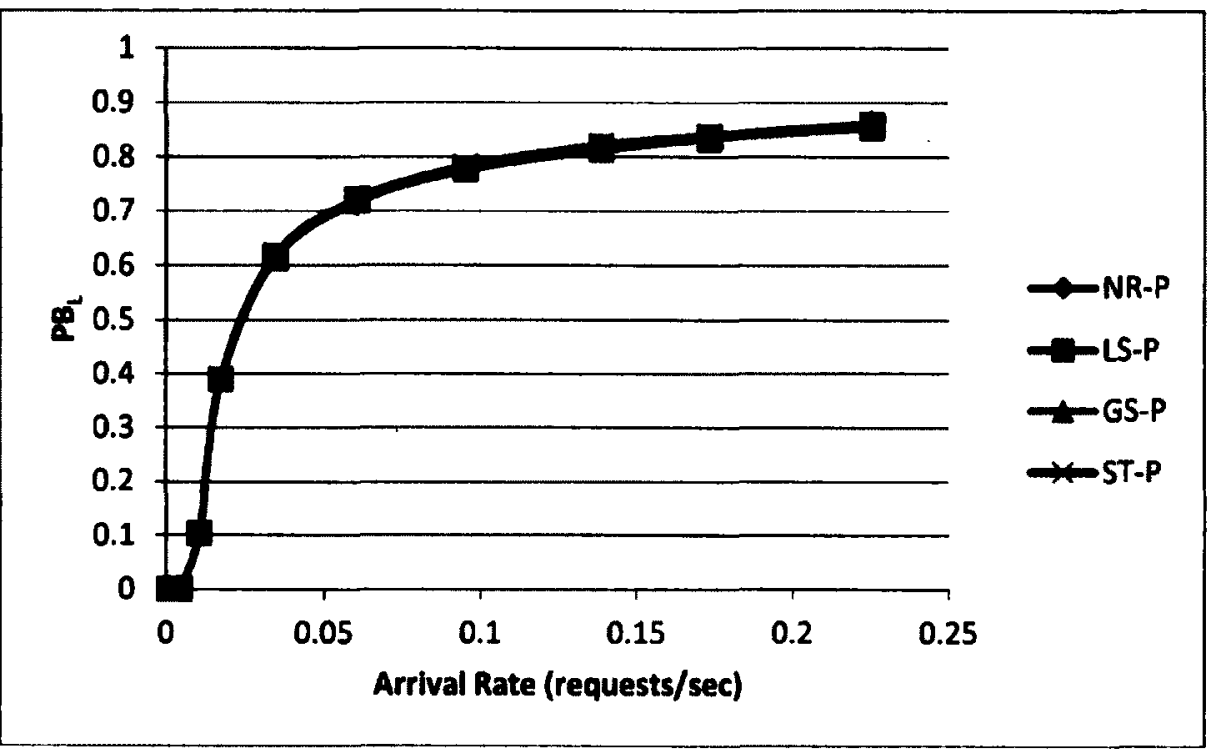

Figure B-1: Impact of the Pre-emption Policles on the Probability of Blocking Low Priority ARs $\left(\mathbf{P B}_{\mathbf{L}}\right)$

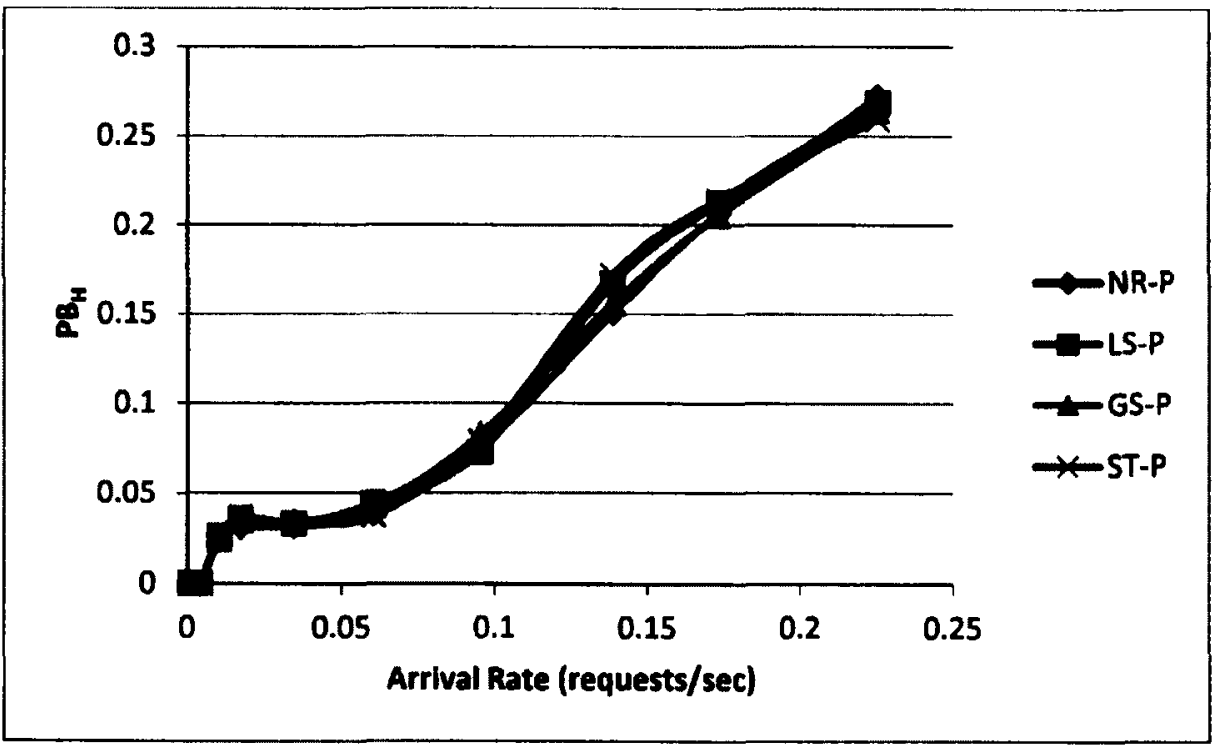

Figure B-2: Impact of the Pre-emption Policies on the Probability of Blocking High Priority ARs $\left(\mathbf{P B}_{\mathbf{H}}\right)$ 


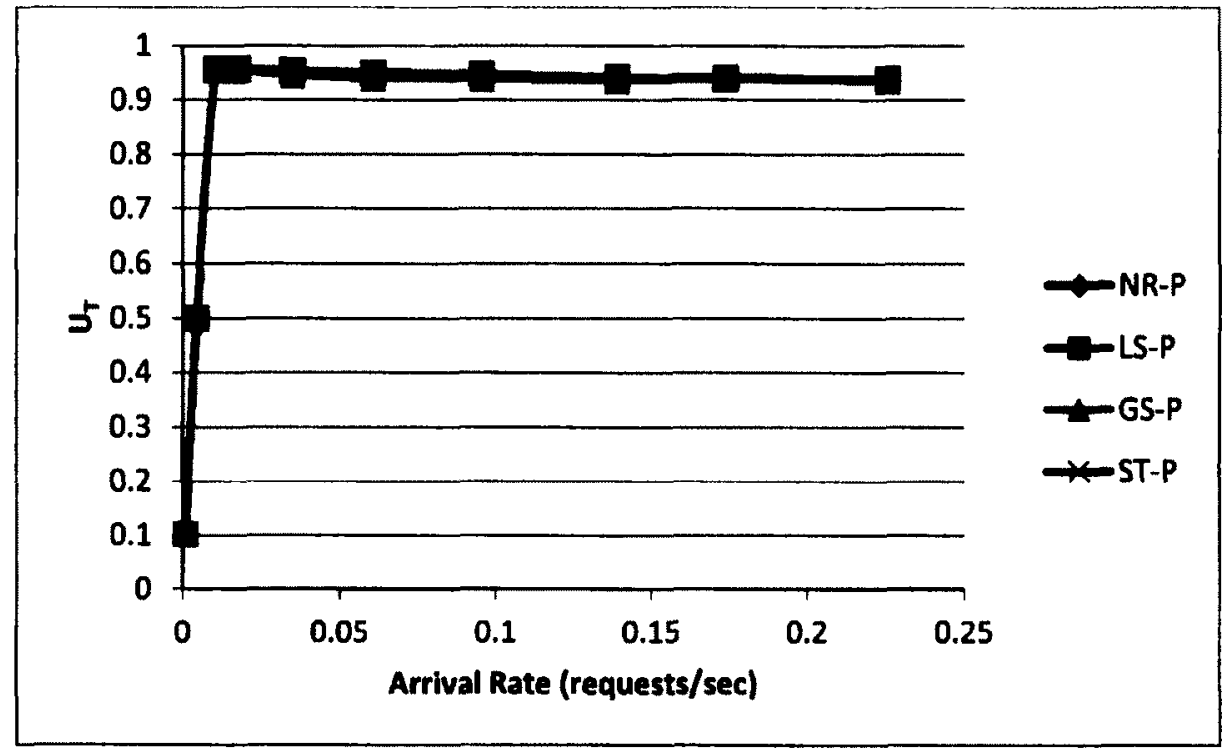

Figure B-3: Impact of the Pre-emption Policies on the Overall Average Resource Utilization $\left(U_{T}\right)$

\section{B.2 Impact of the Number of Resources}

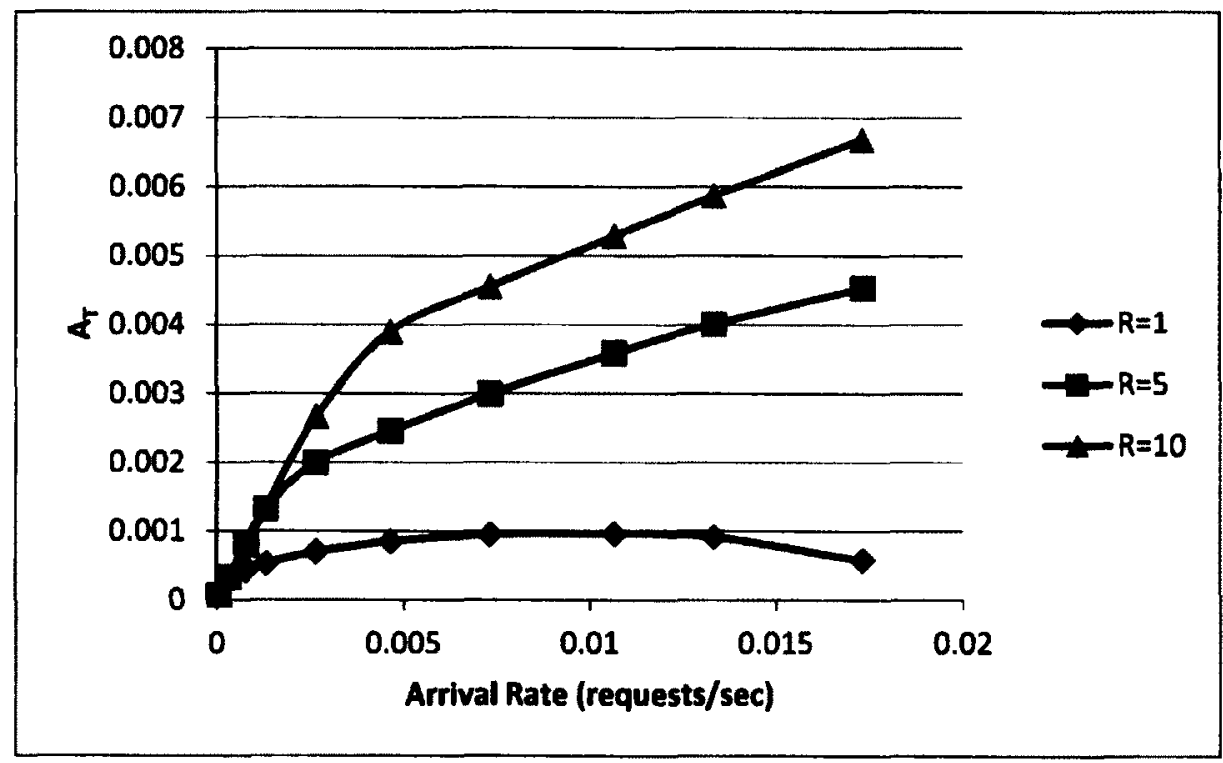

Figure B-4: Impact of the Number of Resources (R) on the Overall Rate of Accepted Requests $\left(A_{T}\right)$ 


\section{B.3 Impact of the Emergency Mean Service Time}

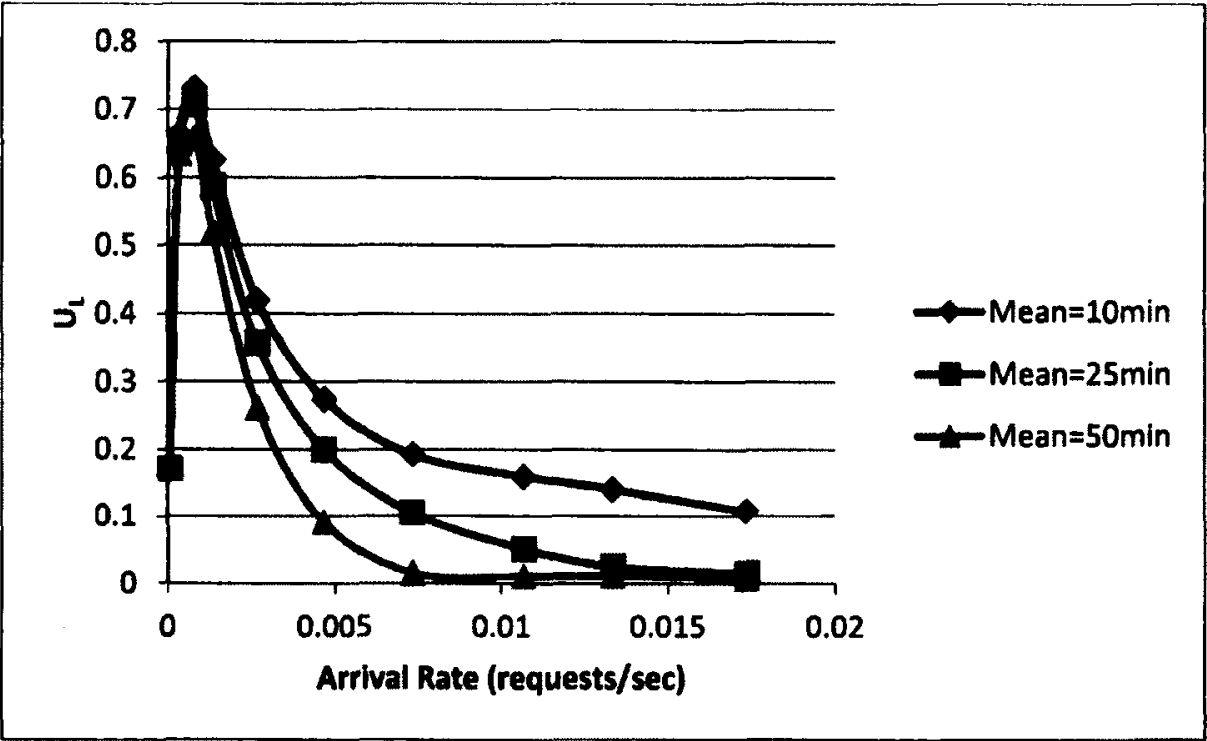

Figure B-5: Impact of the Mean Emergency Service Time on the Average Resource Utilization due to Low Priority ARs $\left(U_{L}\right)$

\section{B.4 Impact of the Proportion of Emergency Requests}

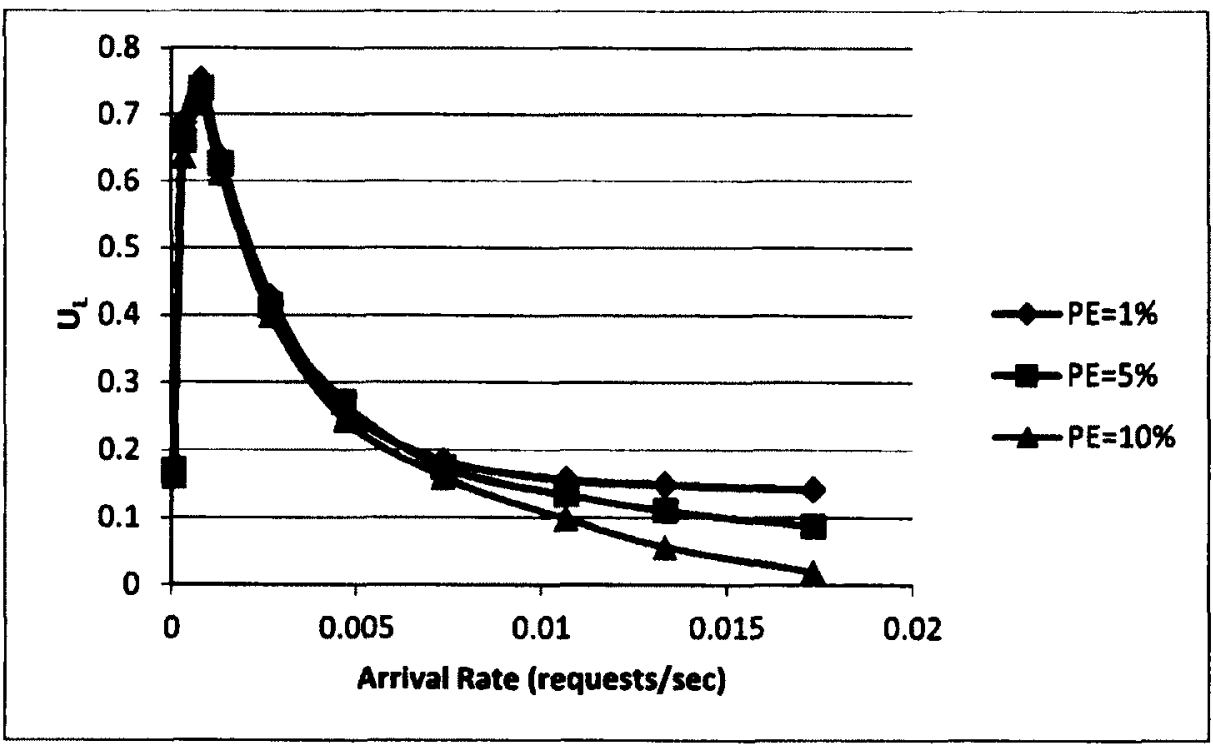

Figure B-6: Impact of the Proportion of Emergencies (PE) on the Average Resource Utilization due to Low Priority ARs ( $\left.U_{L}\right)$ 


\section{B.5 Impact of Padding Time}

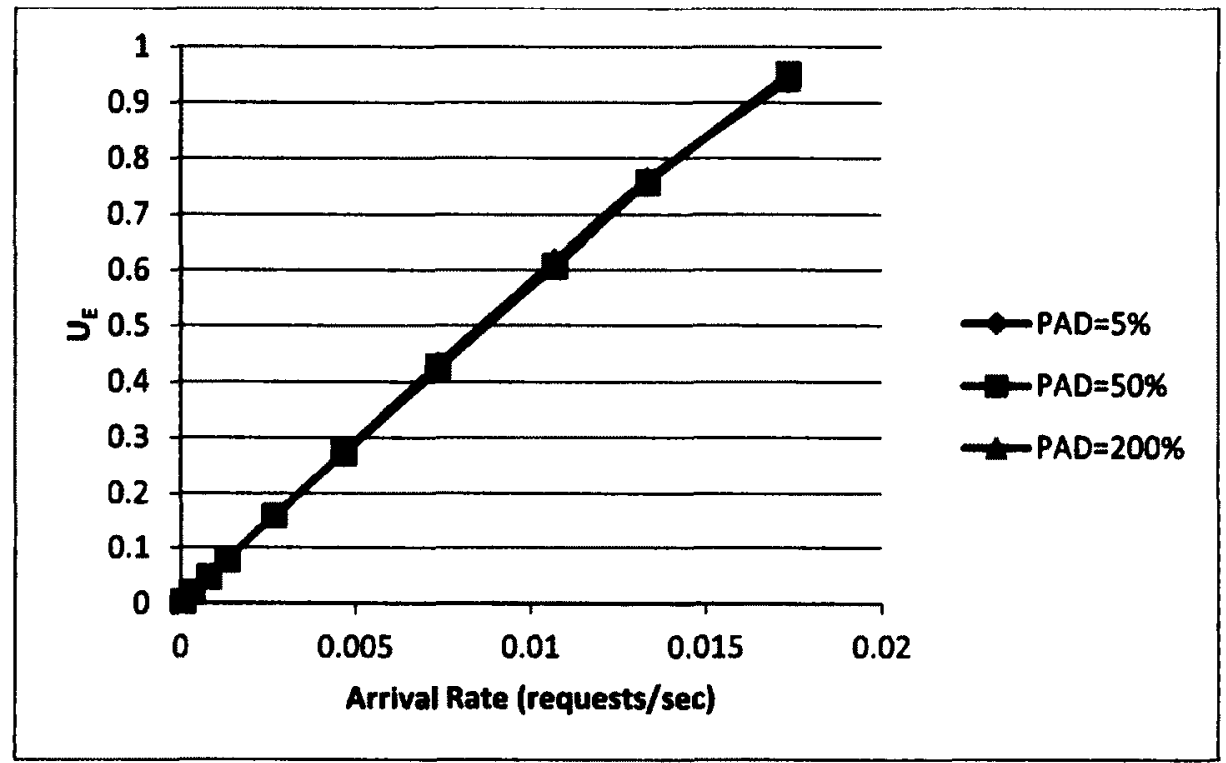

Figure B-7: Impact of Padding on the Average Resource Utilization due to Emergencies $\left(U_{E}\right)$ 